\title{
A numerical classification of the genera Streptomyces and Streptoverticillium using miniaturized physiological tests
}

\author{
PETER KÄMPFER, ${ }^{*}{ }^{*}$ REINER M. KROPPENSTEDT ${ }^{2}$ and WOLFGANG DOTT ${ }^{1}$ \\ ${ }^{1}$ Fachgebiet Hygiene, Technische Universität Berlin, Amrumerstr. 32, D-1000 Berlin 65, Federal Republic of Germany \\ ${ }^{2}$ Deutsche Sammlung von Mikroorganismen und Zellkulturen, Marscheroderweg 1b, D-3300 Braunschweig, \\ Federal Republic of Germany
}

(Received 26 October 1990; accepted 13 February 1991)

\begin{abstract}
Eight hundred and twenty-one strains of the genera Streptomyces and Streptoverticillium were physiologically characterized using a total of 329 miniaturized tests. Overall similarities of all strains were determined by numerical taxonomic techniques using the UPGMA algorithm and the $S_{\mathrm{SM}}$ and the $S_{\mathrm{J}}$ coefficients as measures of similarity. Test error was within acceptable limits. Comparison of photometric and visual test reading revealed overall differences of $\mathbf{7 . 4 5} \%$. A total of 15 major clusters (six or more strains), 34 minor clusters (less than six strains) and 40 single-member clusters were defined at the $81.5 \%$ similarity level $\left(S_{\mathrm{SM}}\right)$. Two clusters containing physiologically, and in some cases morphologically and genetically, different groups could be further subdivided at the $84.0 \%$ similarity level $\left(S_{\mathrm{SM}}\right)$. Generally, similar groupings were obtained with the Jaccard coefficient at similarity levels ranging from $59.6 \%$ to $64.6 \%$ similarity $\left(S_{\mathrm{J}}\right)$, with changes in the definition of clusters and subclusters. The cophenetic correlation coefficients $r_{\mathrm{cs}}$ for the UPGMA $/ S_{\mathrm{J}}$ and the UPGMA $/ S_{\mathrm{SM}}$ analysis were 0.6929 and 0.8239 , respectively. Several phena showed significant overlap with others, indicating the physiological variability within the species. The phenetic data in most cases confirm the major phena of the study of Williams $e t$ al. (1983), Journal of General Microbiology 129, 1743-1813 (although the cluster-groups defined in that study could only be detected in part) and the results indicate that the genus Streptomyces is still overspeciated. Some of the major groupings obtained were very much in line with chemotaxonomic and genetical data. However, several clusters containing only a few strains should be regarded as preliminary 'species' until further information is available. The majority of Streptoverticillium strains presently assigned to different species formed a homogeneous subcluster defined at the $84.0 \%$ similarity level $\left(S_{\mathrm{SM}}\right)$. Thus, on the basis of numerical phenetic and (published) molecular genetic and chemotaxonomic data, our study supports the suggestion that members of the genus Streptoverticillium be reclassified into the genus Streptomyces.
\end{abstract}

\section{Introduction}

The genera Streptomyces and Streptoverticillium are classified within the family Streptomycetaceae and contain Gram-positive aerobic, sporeforming actinomycetes, producing extensive branching substrate and aerial mycelia with LL-diaminopimelic acid and glycine but no characteristic sugar in the wall (wall type I sensu Lechevalier \& Lechevalier, 1970 $a, b$ ). They are widely distributed in nature, play an important role in the turnover of organic matter and are of considerable industrial importance (Kutzner, 1981; Williams et al.,

Abbreviations: OTU, operational taxonomic unit; p-NA, p-nitroanilide; p-NP, p-nitrophenyl; RA, Retinaculiaperti, RF, Rectiflexibilis; SP, Spirales (spore chain categories).
1984; Goodfellow et al., 1984). Studies on the chemotaxonomy of various genera of the Actinomycetales have revealed the value of chemical markers as differentiating characters at the genus level and even at higher taxonomic levels (Collins \& Jones, 1981; Goodfellow \& Cross, 1984; Kroppenstedt, 1985; Lechevalier \& Lechevalier, 1970 $a, b$; Lechevalier et al., 1977; Minnikin \& Goodfellow, 1980; Minnikin et al., 1977; Stackebrandt, 1986). However, although variation of fatty acid and mycolic acid patterns has been found within the genera Streptomyces and Streptoverticillium, chemotaxonomic data are only of limited importance for species differentiation (Minnikin et al., 1978; Goodfellow \& Schaal, 1979; Goodfellow \& Wayne, 1982; Kroppenstedt, 1985).

Studies of rRNA catalogues have shown the Streptomycetaceae to be a coherent taxon (Goodfellow \& Cross, 
1984; Stackebrandt \& Woese, 1981; Stackebrandt et al., 1981). Because of the similarity between species in chemotaxonomic characters, numerical taxonomy studies have been very important to the taxonomy of streptomycetes (Alderson et al., 1984). The extensive numerical classification of Williams et al. (1983a) using 394 type cultures of Streptomyces tested for 139 unit characters was the foundation of an extensive species reduction within the genus Streptomyces. In this study 23 major, 20 minor and 25 single-member clusters, containing 219,46 and 25 validly described species, respectively, were defined at the $77.5 \%$ or $81 \% S_{\mathrm{SM}}$ or $63 \% S_{\mathrm{J}}$ similarity levels. This study forms the basis of the classification in Bergey's Manual of Systematic Bacteriology, vol. 4 (Williams et al., 1989) and provides an invaluable framework for streptomycete taxonomy, including identification (Williams et al., 1983b; Langham et al., 1989).

The current classification of the genus Streptoverticillium (Locci \& Schofield, 1989) is also based on a numerical taxonomic study: 111 strains were tested with 185 unit characters (Locci et al., 1981), and were grouped into 10 multi-membered and 14 single-member clusters. Identification of species can also be achieved by numerical procedures (Williams et al., 1985).

Current improvements in streptomycete taxonomy are mainly concerned with the use of chemotaxonomic, molecular genetic and serological data (Fierro et al., 1987; Mordarski et al., 1986; Saddler et al., 1987; O'Donnell, 1988; Ridell et al., 1986; Gladek et al., 1985) to evaluate the species defined by the numerical studies. Chemotaxonomic methods have still to be improved for the analysis of quantitative data (O'Donnell, 1988). Further aims in streptomycete taxonomy and identification are the evaluation of the taxonomic position of 'species' which have not been part of a natural classification ('species' currently classified into species category IV of Williams et al., 1989) and the development of rapid tests for presumptive identification of unknown isolates.

Although miniaturized tests have gained wide acceptance in diagnostic microbiology, these tests have only rarely been applied to members of the Actinomycetales. In an initial approach, Goodfellow et al. (1987) studied the production of extracellular enzymes by 88 streptomycetes representing 19 numerically defined species of Williams et al. (1983a) and found different patterns of enzyme activity within each of the groups.

The aim of our study was to evaluate the species and species-groups defined by Williams et al. (1983a) and Locci et al. (1981) in an extensive numerical study, and to compare the results with those obtained by other methods. Furthermore, the taxonomic position of many Streptomyces strains not included in the study of
Williams et al. (1983a) and currently classified in species category IV (Williams et al., 1983a) was evaluated.

\section{Methods}

Bacterial strains. A total of 821 strains of the genera Streptomyces and Streptoverticillium were used in the study. All strains were reference organisms from the Deutsche Sammlung von Mikroorganismen und Zellkulturen (DSM), Braunschweig, FRG, from which 420 strains were ISP cultures, and 363 strains were ones used in the study of Williams $e t$ al. $(1983 a)$. Of these cultures, 115 were type strains of validly described species (Williams et al., 1989). They are listed in detail in Table 1. All strains were cultivated on glucose/yeast extract/malt extract agar (GYM) containing the following constituents $\left(\mathrm{g} \mathrm{l}^{-1}\right)$ : yeast extract, 4; malt extract, $10 ; \mathrm{D}$-glucose, $4 ; \mathrm{CaCO}_{3}, 2$; agar, 15 . They were incubated aerobically at $28^{\circ} \mathrm{C}$.

Biochemical tests. Each strain was examined for 329 unit characters, including carbon source utilization tests, sugar fermentation tests and qualitative enzyme tests using chromogenic substrates. Tests were performed in standard microtitration plates (Greiner, Nürtingen, FRG). All tests were read both visually and photometrically.

Carbon source utilization was tested in a mineral medium containing the following constituents $\left(\mathrm{g} \mathrm{l}^{-1}\right): \mathrm{K}_{2} \mathrm{HPO}_{4}, 1 \cdot 74 ; \mathrm{KH}_{2} \mathrm{PO}_{4}, 1.36$; $\left(\mathrm{NH}_{4}\right)_{2} \mathrm{SO}_{4}, 5 \cdot 0 ; \mathrm{MgSO}_{4} .7 \mathrm{H}_{2} \mathrm{O}, 0.5 ; \mathrm{CaCl}_{2} .2 \mathrm{H}_{2} \mathrm{O}, 0.1 ; \mathrm{NaCl}, 9 \cdot 0$; yeast extract (Oxoid), 0.02; bio-lactysate (bio-Merieux), 0.02; vitamin solution, $5 \mathrm{ml}$ [containing $\left(\mathrm{mg} \mathrm{1}^{-1}\right)$ : calcium pantothenate, 0.1 ; nicotinic acid, 0.1 ; biotin, 0.005 ; cyanocobalamin, 0.005 ; folic acid, $0 \cdot 1$; pyridoxine, $0 \cdot 1 ; p$-aminobenzoic acid, $0 \cdot 1$; thiamin pyrophosphate, $0 \cdot 1$; inositol, $0 \cdot 1$; thiamin, $0 \cdot 1$; riboflavin, $0 \cdot 1$ ]; trace element solution, $1 \mathrm{ml}$ [containing $\left(\mathrm{mg} \mathrm{l}^{-1}\right): \mathrm{H}_{3} \mathrm{BO}_{4}, 0.5 ; \mathrm{CuSO}_{4} .5 \mathrm{H}_{2} \mathrm{O}, 0.04 ; \mathrm{KI}, 0 \cdot 1$; $\mathrm{FeCl}_{3} \cdot 6 \mathrm{H}_{2} \mathrm{O}, 0 \cdot 2 ; \mathrm{MnSO}_{4} \cdot \mathrm{H}_{2} \mathrm{O}, 0 \cdot 2 ; \mathrm{Na}_{2} \mathrm{MoO}_{4} \cdot 2 \mathrm{H}_{2} \mathrm{O}, 0 \cdot 2 ;$ $\left.\mathrm{ZnSO}_{4} \cdot 7 \mathrm{H}_{2} \mathrm{O}, 0 \cdot 4\right]$. After adding the different carbon sources, at a final concentration of $0.2 \%(w / v)$, or $0.05 \%(w / v)$ for aromatic compounds, the media were sterilized by filtration. The utilization of 240 compounds was tested (Table 2).

Qualitative enzyme tests were done in filter-sterilized medium (pH 7.2) containing $0.05 \mathrm{M}-\mathrm{Tris} / \mathrm{HCl}$ buffer and $0.05 \%(\mathrm{w} / \mathrm{v})$ each of yeast extract (Oxoid) and bio-lactysate (bio-Merieux). Filter-sterilized solutions of chromogenic substrates [p-nitrophenyl-(p-NP-) linked substrates] were added at final concentrations of $2 \mathrm{~mm}$. For $p$ nitroanilides ( $p$-NA), concentrations of $1 \mathrm{~mm}$ were used. Hydrolysis of 42 chromogenic substrates was tested (Table 2).

Sugar fermentation tests were done in the medium of Hugh and Leifson (1953). A total of 47 different sugars $(1 \%, w / v)$ were tested (Table 2).

To obtain homogeneous suspensions for inoculation of the different test wells, all strains were cultivated in liquid GYM medium for $72 \mathrm{~h}$ at $28^{\circ} \mathrm{C}$. After growth, the cell agglomerates were mechanically homogenized using a sterile Ultraturrax (Jahnke \& Kunkel, Staufen, FRG) for $1 \mathrm{~min}$ at 4000 r.p.m. From this homogenate, $100 \mu$ laliquots were transferred to $5 \mathrm{ml}$ of $0.9 \%(\mathrm{w} / \mathrm{v}) \mathrm{NaCl}$ and each test well was filled with $50 \mu$ lof the bacterial suspension. Test plates were covered with plastic seals (Flow Laboratories) and incubated at $28^{\circ} \mathrm{C}$ for $14 \mathrm{~d}$. Test results were read photometrically with a Multiscan MCC 340 photometer (Flow Laboratories). A carbon substrate utilization test was considered positive if $A_{414}$ (test) $-A_{414}$ (assimilation control) $>0.05$, and a qualitative enzyme test was considered positive if $A_{414}$ (test) $-A_{414}$ (chromogenic substrate control) $>0 \cdot 3$. All test results were checked visually.

Coding of data. All of the characters were scored plus (1) or minus (0) and then examined using a cluster program written in FORTRAN. Clustering was achieved with the unweighted average linkage 
(UPGMA) algorithm (Sneath \& Sokal, 1973) using the simple matching coefficient $\left(S_{\mathrm{SM}}\right)$ and the Jaccard coefficient $\left(S_{\mathrm{J}}\right)$. Overlap of clusters was calculated between all pairs of major and minor clusters using the formulas given in the ovcLusT program (Sneath, 1979). The chosen critical value of overlap $V_{0}$ was set at $5 \%$, according to Williams et al. $(1983 a)$. The cophenetic correlation coefficient $\left(r_{\mathrm{cs}}\right)$ as a measure of agreement between the similarity values implied by a phenogram and those of the original similarity matrix were calculated for both classifications according to Sneath \& Sokal (1973).

Test reproducibility. All tests were performed in triplicate for 20 randomly chosen strains (Table 1 ), and an estimate of test variance $\left(s_{i}{ }^{2}\right)$ and the average probability $\left(p_{t i}\right)$ of an erroneous result were calculated according to the formulae of Sneath \& Johnson (1972).

Computing. All calculations were done on a personal computer (Compaq Deskpro AT 80386).

\section{Results}

\section{Test error and final data-matrix}

Test error was estimated for visually-read test results by calculation of the pooled variances and the average probability of an erroneous result. For 20 strains grouped into different species or species-groups by Williams $e t$ al. $(1983 a$ ), all tests were performed in triplicate (Table 1). The average probability $\left(p_{\mathrm{ti}}\right)$ of an erroneous result for all tests $(n=329)$ was $3.11 \%$. Within the test groups, significant differences in reproducibility were found. Carbon substrate utilization tests $(n=240)$ gave a $p_{\mathrm{ti}}$ of $2.71 \%$, qualitative enzyme tests $(n=42)$ a $p_{\mathrm{ti}}$ of $1.14 \%$, and tests for acid formation from various sugars $(n=47)$ a $p_{\mathrm{ti}}$ of $7.09 \%$. A comparison of visual and photometric test readings for all strains (821) and all tests (329) gave overall discrepancies of $7.45 \%$. The discrepancies varied from $0 \%$ to $66 \%$. The most obvious deviations were found for the substrate utilization tests of the aromatic compounds 3-hydroxyphenylacetate and protocatechuate. Several strains produced a brown colouration of the test media independent of growth. Additionally, the tests for acid formation from different sugars showed high inconsistencies with respect to differences between visual and photometric readings. Small differences were found for the qualitative enzyme tests using chromogenic substrates.

Because of the different growth characteristics of the 20 strains chosen for reproducibility studies, and their great variability in several features (speed and homogeneity of growth, etc.), it was not possible to determine the extent of test variations due to different degrees of uniformity of the test organisms themselves, or to unknown and uncontrolled factors that affect test responses of most or all test organisms. Therefore we did not exclude tests in advance from the final data matrix, which contained 821 strains against 329 unit characters. As in the study of Williams et al. (1983a), the data shown in Tables 2 to 4 should serve as a reference source. Diagnostic properties were selected using computer programs and a probability matrix was constructed for identification purposes. This matrix contained characters of high consistency and stability within the taxa (see the accompanying paper: Kämpfer \& Kroppenstedt, 1991). The following sections refer exclusively to the visually checked test results.

\section{Clustering of strains using the UPGMA algorithm with the $S_{S M}$ coefficient and characterization of taxa}

A simplified phenogram of the $S_{\mathrm{SM}} / \mathrm{UPGMA}$ analysis is given in Fig. 1. The classification based on the $S_{\mathrm{SM}} / \mathrm{UPGMA}$ analysis gave compact clusters in a homogeneous phenogram. The cophenetic correlation coefficient $r_{\text {cs }}$ as a measure of correlation between corresponding pairs from the similarity matrix and the cophenetic matrix was 0.8239 .

Because of the numerous tests performed, from 1653 possible overlapping clusters and subclusters (singlemember clusters were not considered in these calculations), a total of 106 pairs of phena showed overlap at a confidence interval of $P=90 \%, 122$ pairs were found at at a confidence interval of $P=95 \%$, and 226 overlapping pairs of phena could be obtained with $P=99 \%$ taking a chosen level of $V_{0}=5 \%$ (Williams et al., 1983a).

Fifteen major clusters (containing six or more strains), 34 minor clusters (containing less than six strains) and 40 single-member clusters were obtained. They were numbered consecutively in ascending similarity, independent of the number of strains they contained. The clusters were defined at a similarity level of $81.5 \%\left(S_{\mathrm{SM}}\right)$. In contrast to the analysis of Williams et al. $(1983 a)$, we did not classify all the different phena into cluster-groups, but two clusters (clusters 1 and 22) could be divided into subclusters at a similarity level of $84.0 \%\left(S_{\mathrm{SM}}\right)$. These subclusters were composed of morphologically and physiologically distinct groups, which could also be differentiated on the basis of genetical and chemotaxonomic data. Several major clusters of the study of Williams et al. (1983a) could be recovered, whereas others (in most cases the smaller ones) could not.

The composition of the different clusters is shown in Table 1. In accordance with the study of Williams $e t$ al. (1983a), phena were named, when possible, after the earliest described 'species' which they contained. The same practice was followed for phena not included in the Approved Lists of Bacterial Names (Skerman et al., 1980). Where more than one type strain was described in the same year, the name was selected alphabetically. In many cases, strains with the same species designations were found in different phena. In these cases, and for clusters which were similar in composition to those of 


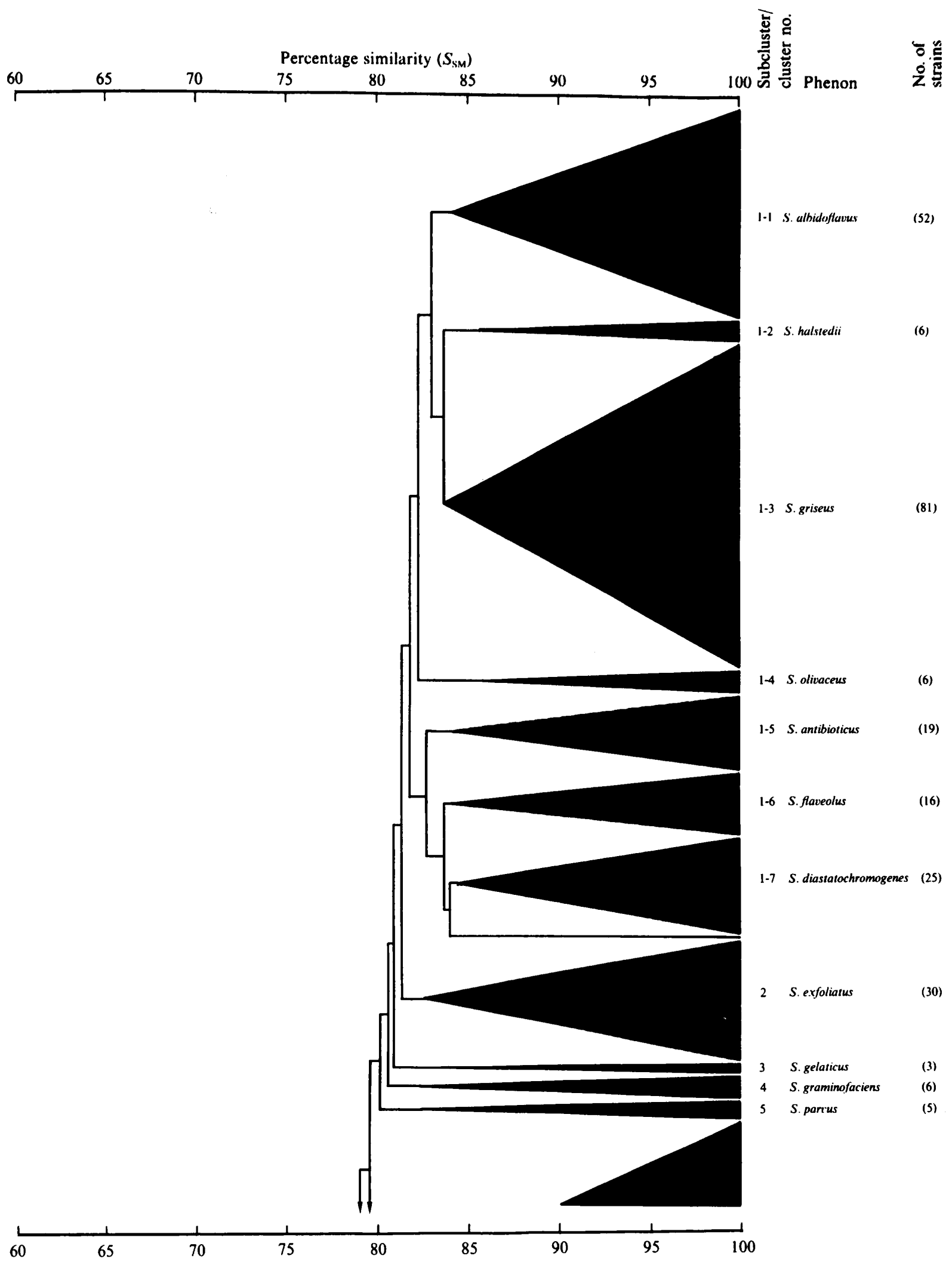

Fig. 1. Simplified phenogram showing the relationship between the phena recovered in the UPGMA $/ S_{\mathrm{SM}}$ analysis. Single-member clusters are not labelled. 


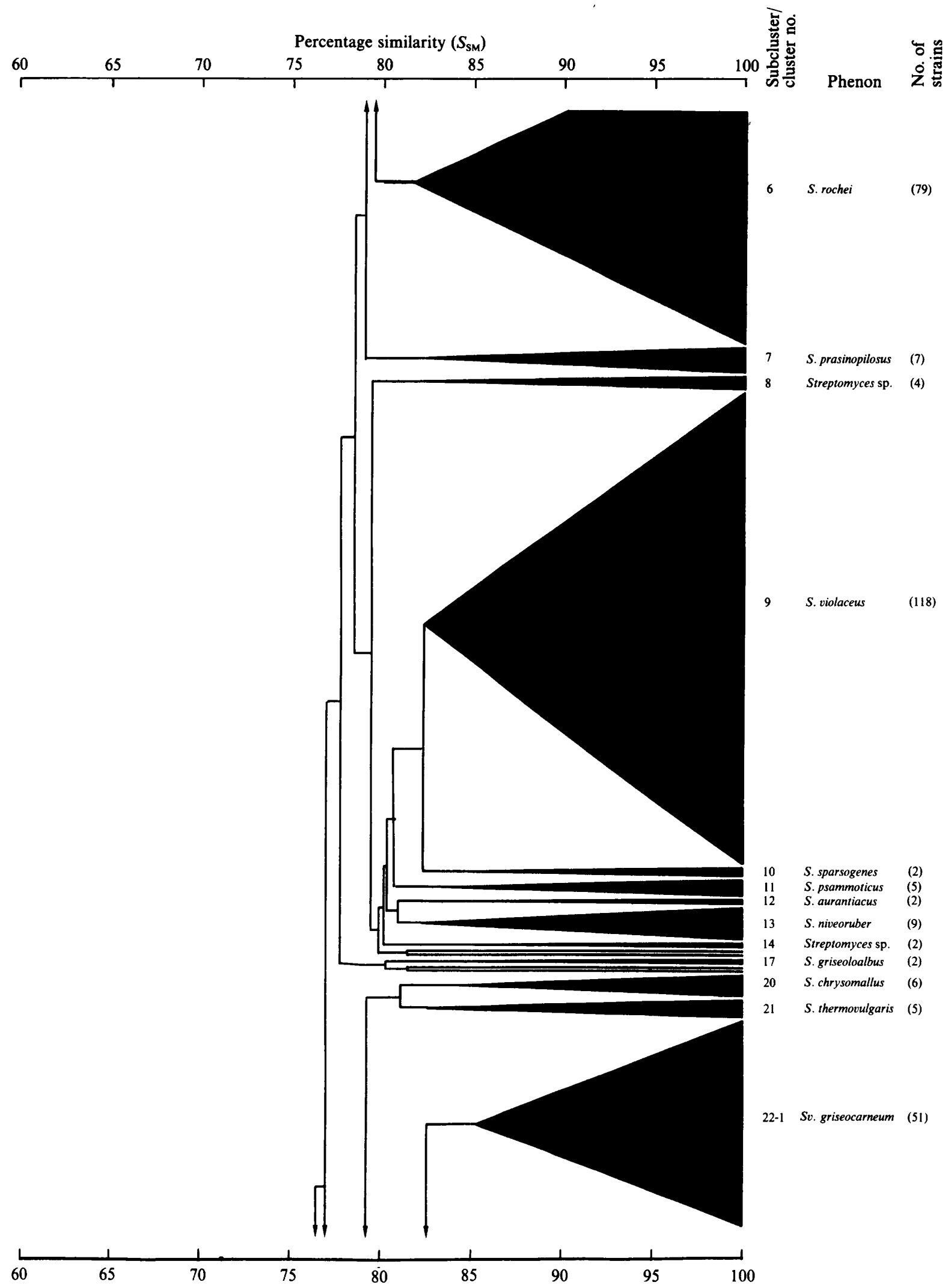




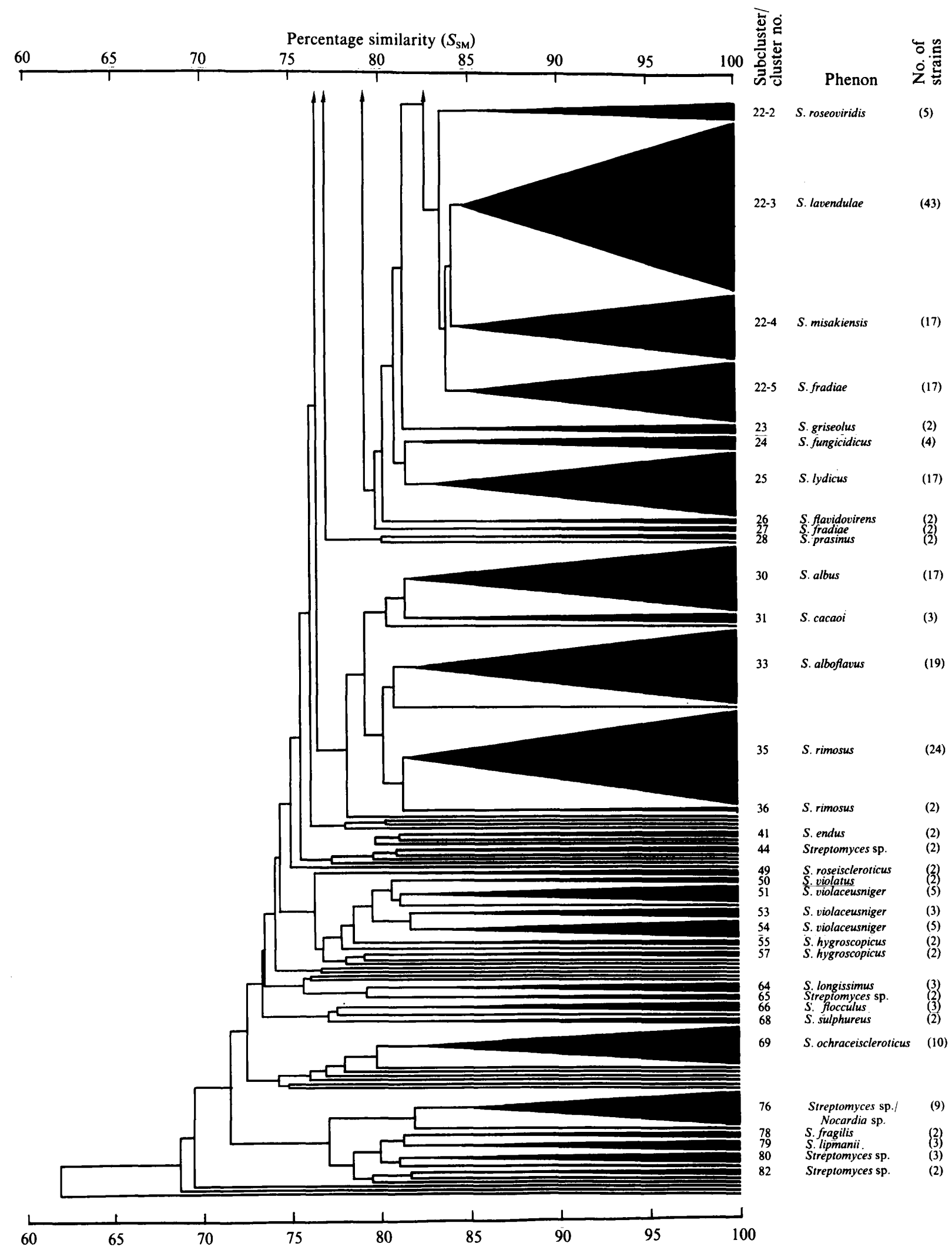


Table 1. Designation and source of cultures assigned to clusters and subclusters of the UPGMA/S $S_{S M}$ analysis

A total of 821 strains are listed, including 115 type strains designated by Williams et al. (1989), 348 type strains of nomenspecies listed in the Approved Lists of Bacterial Names (Skerman et al., 1980) and 420 ISP cultures (Shirling \& Gottlieb, 1968 $a, b, 1969,1972)$. Clusters were defined at the $81 \cdot 5 \%$ similarity level $\left(S_{\mathrm{SM}}\right)$. Clusters 1 and 22 were subdivided into subclusters at a similarity level of $84 \cdot 0 \%\left(S_{\mathrm{SM}}\right)$.

\begin{tabular}{|c|c|c|c|c|c|}
\hline$s_{\mathrm{SM}}^{\dagger}$ & $S_{\mathrm{J}} \ddagger$ & Strain designation $\S$ & Strain numbers\| & $\begin{array}{l}\text { Williams } \\
\text { group }\end{array}$ & $\begin{array}{l}\text { Bergey category } \\
\text { and species no. }+\dagger\end{array}$ \\
\hline
\end{tabular}

Strains assigned to cluster 1: Streptomyces albidoflavus/S. griseus/S. antibioticus

Strains assigned to subcluster 1-1: Streptomyces albidoflavus (52)

\begin{tabular}{|c|c|c|}
\hline $1-1$ & $1-1$ & S. albidoflavus \\
\hline $1-1$ & $1-1$ & S. albidoflavus \# \\
\hline $1-1$ & $1-1$ & S. albidus \\
\hline $1-1$ & $1-1$ & S. albus \\
\hline $1-1$ & $1-1$ & S. albus \\
\hline $1-1$ & $1-1$ & S. albus \\
\hline $1-1$ & $1-1$ & S. alni \\
\hline $1-1$ & $1-1$ & S. badius \\
\hline $1-1$ & $1-1$ & S. candidus \\
\hline $1-1$ & $1-1$ & S. canescens \\
\hline $1-1$ & $1-1$ & S. cellulosae \\
\hline $1-1$ & $1-1$ & S. champavatii \\
\hline $1-1$ & $1-1$ & S. citreus \\
\hline $1-1$ & $1-1$ & S. coelicolor \\
\hline $1-1$ & $1-1$ & S. coelicolor \\
\hline $1-1$ & $1-1$ & S. coelicolor \\
\hline $1-1$ & $1-1$ & S. coelicolor \\
\hline $1-1$ & $1-1$ & S. coelicolor \\
\hline $1-1$ & $1-1$ & S. coelicolor \\
\hline $1-1$ & $1-1$ & S. coelicolor \\
\hline $1-1$ & $1-1$ & S. coelicolor \\
\hline $1-1$ & $1-1$ & S. coelicolor \\
\hline $1-1$ & $1-1$ & S. coelicolor \\
\hline $1-1$ & $1-1$ & S. coelicolor \\
\hline $1-1$ & $1-1$ & S. collinus \\
\hline $1-1$ & $1-1$ & S. coriofaciens \\
\hline $1-1$ & $1-1$ & S. diastaticus \# \\
\hline $1-1$ & $1-1$ & S. felleus \\
\hline $1-1$ & $1-1$ & S. globisporus \\
\hline $1-1$ & $1-1$ & S. globisporus subsp. caucasicus \\
\hline $1-1$ & $1-1$ & S. gougerotii \\
\hline $1-1$ & $1-1$ & S. griseus \\
\hline $1-1$ & $1-1$ & S. griseus \\
\hline $1-1$ & $1-1$ & S. griseus \\
\hline $1-1$ & $1-1$ & S. griseus \\
\hline $1-1$ & $1-1$ & S. griseus \\
\hline $1-1$ & $1-1$ & S. griseus subsp. solvifaciens \\
\hline $1-1$ & $1-1$ & S. hominis \\
\hline $1-1$ & $1-1$ & $S$. intermedius \\
\hline $1-1$ & $1-1$ & S. krainskii \\
\hline $1-1$ & $1-1$ & S. levoris \\
\hline $1-1$ & $1-1$ & S. limosus \\
\hline $1-1$ & $1-1$ & S. ochroleucus \\
\hline $1-1$ & $1-1$ & S. odorifer \\
\hline $1-1$ & $1-1$ & S. prunicolor \\
\hline $1-1$ & $1-1$ & S. rutgersensis \\
\hline $1-1$ & $1-1$ & S. sampsonii \\
\hline $1-1$ & $1-1$ & S. saprophyticus \\
\hline $1-1$ & $1-1$ & S. somaliensis \\
\hline $1-1$ & $1-1$ & Streptomyces sp. \\
\hline $1-1$ & $1-1$ & Streptomyces sp. \\
\hline $1-1$ & $1-1$ & S. tetanusemus \\
\hline
\end{tabular}

${ }^{*}$ DSM 40792

DSM $40455^{T}$ NRRL B-2663

DSM 40869

*DSM 40890

*DSM 40891

*DSM 40671

DSM 40557

* DSM 40139T

DSM 40838

*DSM 40001(T)

*DSM 40802

*DSM $40841^{\mathrm{T}}$

DSM 40364

*DSM 40233(T)

*DSM 40623

*DSM 40624

*DSM 40675

*DSM 40678

* DSM 40665

*DSM 40679

*DSM 40680

-DSM 40681

*DSM 40682

*DSM 40683

*DSM 40909

DSM 40485

*DSM 40496 ${ }^{\mathrm{T}}$

*DSM 40130(T)

*DSM 40991

*DSM 40814

*DSM 40324(T)

*DSM 40661

*DSM 40882

*DSM 40931

*DSM 40984

* DSM 40983

*DSM 40933

DSM 40770

*DSM 40372(T)

DSM 40321

DSM 40202

*DSM $40131^{(\mathrm{T})}$
DSM 40591

*DSM 40347(T)
*DSM 40335T

*DSM 40077(T)

*DSM 40394(T)

DSM 40537

*DSM 40267

DSM 40622

DSM 40872

DSM 40585

ATCC 4838

ATCC 3313

NRRL B-5682

IMRU 3570

ATCC 21553

ATCC 19907
NRRL B-2663
ATCC 25422 ISP 5455

ATCC 27415 ISP 5557

ATCC 19888 ISP 5139

ATCC 19736 ISP 5001

ATCC 25441 ISP 5364

ATCC 23899 ISP 5233

ATCC 14155 ISP 5485 ATCC 3315 ISP 5496

ATCC 19752 ISP 5130

ATCC 10975 ISP 5324

$\begin{array}{cccc}\text { Strains assigned to subcluster 1-2: Streptomyces halstedii (6) } \\ 1-2 & 15 & \text { S. flavogriseus } & \text { *DSM 40323(1) }\end{array}$

1-2 $\quad 15 \quad$ S. flavidovirens

1-2 15 S. griseolus

1-2 $15 \quad S$. halstedii \#

1-2 15 S. nigrifaciens

1-2 15 S. scabies
CC 25452 ISP 5323

*DSM 40062(T) ATCC 3320 ISP 5062

${ }^{*}$ DSM $40067^{(T)}$ ATCC 3325 ISP 5067

${ }^{*}$ DSM 40068 $\quad$ ATCC 10897 ISP 5068

${ }^{*}$ DSM 40071(T) ATCC 19791 ISP 5071

DSM 40859 IFO 3111
Category I Species no. 01

Category I Species no. 09

Category I Species no. 09

Category I Species no. 09

A 08

C Sm Category III Species no. 15

A 1A Category I Species no. 01

A $1 \mathrm{~A}$

A $1 \mathrm{~A}$

Category IV Species no. 02 (yellow series)

Category I Species no. 01

Category I Species no. 01

Category I Species no. 01

Category I Species no. 01

Category I Species no. 01

Category I Species no. 01

Category I Species no. 01

Category I Species no. 01

Category I Species no. 01

Category I Species no. 01

Category I Species no. 01

Category I Species no. 11

A 19 Category I Species no. 12

A 1A Category I Species no. 01

Category I Species no. 02

Category I Species no. 02

A 1A Category I Species no. 01

Category I Species no. 02

Category I Species no. 02

Category I Species no. 02

Category I Species no. 02

Category I Species no. 02

Category I Species no. 02

A 1A Category I Species no. 01

A $1 \mathrm{~A}$

A 1A Category I Species no. 01

A 1A Category I Species no. 01

A 11 Category III Species no. 01

A 1A Category I Species no. 01

A 1A Category I Species no. 01

A $1 \mathrm{~A}$

Category IV Species no. 08 (white series)

A 1A

A 1C Category I Species no. 03

A 1C Category I Species no. 03

A 1C Category I Species no. 03

A 1C Category I Species no. 03

A 1C Category I Species no. 03 
Table 1 (continued)

\begin{tabular}{|c|c|c|c|c|c|c|c|}
\hline$S_{\text {SM }} \dagger$ & $S_{\mathrm{J}} \ddagger$ & Strain designation $\S$ & \multicolumn{2}{|c|}{ Strain numbers $\|$} & \multirow[t]{2}{*}{$\begin{array}{l}\text { Williams } \\
\text { group }\end{array}$} & \multicolumn{2}{|r|}{$\begin{array}{l}\text { Bergey category } \\
\text { and species no. }+\dagger\end{array}$} \\
\hline \multicolumn{7}{|c|}{ Strains assigned to subcluster 1-3: Streptomyces griseus (81) } & \\
\hline $1-3$ & $1-2$ & S. acidoresistans & DSM 40540 & ATCC 27413 ISP 5540 & & & \\
\hline $1-3$ & 10 & S. acrimycini & ${ }^{*}$ DSM $40135^{\top}$ & ATCC 19885 ISP 5135 & & Category IV & Species no. 04 (green series) \\
\hline $1-3$ & $1-2$ & S. albidus & DSM 40320 & ATCC 25423 ISP 5320 & A $1 \mathbf{B}$ & & \\
\hline $1-3$ & $1-2$ & S. albidus & DSM 40793 & NRRL B-1672 & & & \\
\hline $1-3$ & 8 & S. albovinaceus & ${ }^{*}$ DSM $40136^{(T)}$ & ATCC 15823 ISP 5136 & A $1 B$ & Category I & Species no. 02 \\
\hline $1-3$ & 8 & S. albovinaceus & - DSM 40796 & ATCC 12951 & & Category I & Species no. 02 \\
\hline $1-3$ & $1-2$ & S. alboviridis & ${ }^{*}$ DSM $40326^{(T)}$ & ATCC 25425 ISP 5326 & A 1 B & Category I & Species no. 02 \\
\hline $1-3$ & $1-20$ & $S$. aureus & DSM 40862 & ATCC 3309 & & & \\
\hline $1-3$ & $1-2$ & S. bacillaris & ${ }^{*}$ DSM $40598^{(\tau)}$ & ATCC 15855 ISP 5598 & A 1 B & Category I & Species no. 02 \\
\hline $1-3$ & 30 & S. californicus & ${ }^{*}$ DSM $40058^{\mathrm{T}}$ & ATCC 3312 ISP 5058 & A 09 & Category II & Species no. 02 \\
\hline $1-3$ & $1-2$ & S. californicus & ${ }^{*}$ DSM 40801 & ATCC 15436 & & Category II & Species no. 02 \\
\hline $1-3$ & $1-2$ & S. cavourensis & ${ }^{*}$ DSM $40300^{(T)}$ & ATCC 14889 ISP 5300 & A $1 B$ & Category I & Species no. 02 \\
\hline $1-3$ & $1-2$ & S. chrysomallus & ${ }^{*}$ DSM $40128^{(T)}$ & ATCC 11523 ISP 5128 & A 1 B & & \\
\hline $1-3$ & $1-2$ & S. citreofluorescens & ${ }^{*}$ DSM $40265^{(T)}$ & ATCC 15858 ISP 5265 & A $1 B$ & Category I & Species no. 02 \\
\hline $1-3$ & $1-2$ & S. cretaceus & DSM 40561 & ATCC 3005 ISP 5561 & A 03 & & \\
\hline $1-3$ & $1-2$ & S. cyaneofuscatus & ${ }^{*}$ DSM $40148^{(T)}$ & ATCC 23619 ISP 5148 & A 1 B & Category I & Species no. 02 \\
\hline $1-3$ & $1-2$ & S. fimicarius & ${ }^{*}$ DSM $40322^{(T)}$ & ATCC 25449 ISP 5322 & A $1 B$ & Category I & Species no. 02 \\
\hline $1-3$ & $1-2$ & S. floridae & ${ }^{*}$ DSM 40938 & NCIB 9345 & & Category IV & Species no. 04 (yellow series) \\
\hline $1-3$ & $1-2$ & S. fluorescens \# & ${ }^{*}$ DSM $40203^{(T)}$ & ATCC 15860 ISP 5203 & A 1 B & Category I & Species no. 02 \\
\hline $1-3$ & $1-2$ & S. fradiae & ${ }^{*}$ DSM 40705 & NRRL 2599 & & Category II & Species no. 18 \\
\hline $1-3$ & $1-2$ & S. fradiae & *DSM 40940 & & & Category II & Species no. 18 \\
\hline $1-3$ & $1-2$ & S. fulvissimus & ${ }^{*}$ DSM 40767 & & & & \\
\hline $1-3$ & $1-20$ & S. fulvoviridis & DSM 40210 & ATCC 15863 ISP 5210 & A 03 & & \\
\hline $1-3$ & $1-2$ & S. globisporus & ${ }^{*} \mathrm{DSM} 40199(\mathrm{~T})$ & ATCC 15864 ISP 5199 & A 1 B & Category I & Species no. 02 \\
\hline $1-3$ & $1-2$ & S. globisporus & *DSM 40871 & & & Category I & Species no. 02 \\
\hline $1-3$ & $1-2$ & S. griseinus & ${ }^{*}$ DSM $40047^{(T)}$ & ATCC 23915 ISP 5047 & A 1 B & Category I & Species no. 02 \\
\hline $1-3$ & $1-2$ & S. griseobrunneus & ${ }^{*}$ DSM $40066^{(T)}$ & ATCC 19762 ISP 5066 & A $1 \mathbf{B}$ & Category I & Species no. 02 \\
\hline $1-3$ & $1-2$ & S. griseus & ${ }^{*}$ DSM $40236^{(T)}$ & ATCC 23345 ISP 5236 & A 1 B & Category I & Species no. 02 \\
\hline $1-3$ & $1-2$ & S. griseus & *DSM 40627 & & & Category I & Species no. 02 \\
\hline $1-3$ & $1-2$ & S. griseus & ${ }^{*}$ DSM 40653 & & & Category I & Species no. 02 \\
\hline $1-3$ & $1-2$ & S. griseus & *DSM 40654 & & & Category I & Species no. 02 \\
\hline $1-3$ & $1-2$ & S. griseus & *DSM 40656 & & & Category I & Species no. 02 \\
\hline $1-3$ & $1-2$ & S. griseus & *DSM 40657 & & & Category I & Species no. 02 \\
\hline $1-3$ & $1-2$ & S. griseus & ${ }^{*}$ DSM 40658 & & & Category I & Species no. 02 \\
\hline $1-3$ & $1-2$ & S. griseus & ${ }^{*}$ DSM 40659 & & & Category I & Species no. 02 \\
\hline $1-3$ & $1-2$ & S. griseus & ${ }^{*}$ DSM 40660 & & & Category I & Species no. 02 \\
\hline $1-3$ & $1-2$ & S. griseus & ${ }^{*}$ DSM 40670 & & & Category I & Species no. 02 \\
\hline $1-3$ & $1-2$ & S. griseus & ${ }^{*}$ DSM 40695 & & & Category I & Species no. 02 \\
\hline $1-3$ & $1-2$ & S. griseus & *DSM 40707 & NRRL 2764 & & Category I & Species no. 02 \\
\hline $1-3$ & $1-2$ & S. griseus & ${ }^{*}$ DSM 40693 & & & Category I & Species no. 02 \\
\hline $1-3$ & $1-2$ & S. griseus & ${ }^{*}$ DSM 40757 & & & Category I & Species no. 02 \\
\hline $1-3$ & $1-2$ & S. griseus & *DSM 40759 & ATCC 15395 & & Category I & Species no. 02 \\
\hline $1-3$ & $1-2$ & S. griseus & * DSM 40855 & ATCC 10137 & & Category I & Species no. 02 \\
\hline $1-3$ & $1-2$ & $S$. griseus & *DSM 40817 & ATCC 10971 & & Category I & Species no. 02 \\
\hline $1-3$ & $1-2$ & S. griseus & * DSM 40878 & & & Category I & Species no. 02 \\
\hline $1-3$ & $1-2$ & S. griseus & *DSM 40881 & ATCC 13968 & & Category I & Species no. 02 \\
\hline $1-3$ & $1-2$ & $S$. griseus & *DSM 40939 & & & Category I & Species no. 02 \\
\hline $1-3$ & $1-2$ & S. griseus subsp. alpha & - DSM $40937^{(T)}$ & NRRL B-2249 & & Category I & Species no. 02 \\
\hline $1-3$ & $1-2$ & S. griseus subsp. farinosus & DSM 40932 & & & Category I & Species no. 02 \\
\hline $1-3$ & $1-2$ & S. halstedii & ${ }^{*}$ DSM 40863 & ATCC 13449 & & Category I & Species no. 03 \\
\hline $1-3$ & $1-2$ & $S$. lavendulae & *DSM 41571 & ATCC 13664 & & Category I & Species no. 22 \\
\hline $1-3$ & $1-2$ & S. lavendulae & *DSM 41572 & ATCC 13665 & & Category I & Species no. 22 \\
\hline $1-3$ & $1-2$ & S. lipmanii & ${ }^{*} \mathrm{DSM} 40070^{(\mathrm{T})}$ & ATCC 3331 ISP 5070 & A $1 \mathbf{B}$ & Category I & Species no. 02 \\
\hline $1-3$ & $1-20$ & S. luteolutescens & DSM 40600 & ATCC 27445 ISP 5600 & A 1 B & & \\
\hline $1-3$ & $1-2$ & S. mediocidicus & DSM 40864 & ATCC 13278 & & & \\
\hline $1-3$ & $1-2$ & $S$. mediocidicus & DSM 40865 & ATCC 13279 & & & \\
\hline $1-3$ & $1-2$ & S. microflavus\# & ${ }^{*}$ DSM $40331^{\mathrm{T}}$ & ATCC 13231 ISP 5331 & A 23 & Category I & Species no. 20 \\
\hline $1-3$ & $1-2$ & S. oligocarbophilus & DSM 40589 & ATCC 27453 ISP 5589 & A $1 B$ & & \\
\hline $1-3$ & 10 & S. olivoviridis & ${ }^{*}$ DSM $40211^{(T)}$ & ATCC 15882 ISP 5211 & A 03 & Category II & Species no. 20 \\
\hline $1-3$ & $1-2$ & S. ornatus & DSM 40307 & ATCC 23265 ISP 5307 & A $1 B$ & & \\
\hline $1-3$ & $1-2$ & $S$. parvus & ${ }^{*}$ DSM $40348^{(T)}$ & ATCC 12433 ISP 5348 & A $1 B$ & Category I & Species no. 02 \\
\hline $1-3$ & $1-2$ & S. pluricolorescens & ${ }^{*}$ DSM 40019(T) & ATCC 19798 ISP 5019 & A $1 B$ & Category I & Species no. 02 \\
\hline $1-3$ & $1-2$ & $S$. praecox & ${ }^{*} \mathrm{DSM} 40393^{\mathrm{T}}$ & ATCC 3374 ISP 5393 & & Category IV & $\checkmark$ Species no. 08 (yellow seri \\
\hline $1-3$ & $1-2$ & S. reticuli & DSM 40776 & ATCC 23384 & & & \\
\hline $1-3$ & 4 & S. roseochromogenes & DSM 40463 & ATCC 13400 ISP 5463 & & & \\
\hline
\end{tabular}


Table 1 (continued)

\begin{tabular}{rrl}
\hline \hline & & \\
$S_{\mathrm{SM}} \dagger$ & $S_{\mathrm{J}} \ddagger$ & \multicolumn{1}{c}{ Strain designatid } \\
\hline $1-3$ & $1-2$ & S. roseochromogenes \\
$1-3$ & 10 & S. scabies \\
$1-3$ & $1-2$ & S. setonii \\
$1-3$ & $1-2$ & S. sindenensis \\
$1-3$ & $1-2$ & S. sindenensis \\
$1-3$ & $1-2$ & Streptomyces sp. \\
$1-3$ & $1-2$ & Streptomyces sp. \\
$1-3$ & $1-2$ & Streptomyces sp. \\
$1-3$ & $1-2$ & Streptomyces sp. \\
$1-3$ & $1-2$ & Streptomyces sp. \\
$1-3$ & $1-2$ & S. streptomycini \\
$1-3$ & $1-2$ & S. vinaceus \\
$1-3$ & 47 & S. viridochromogenes \\
$1-3$ & $1-2$ & S. vulgaris \\
$1-3$ & $1-2$ & S. willmorei \\
$1-3$ & $1-2$ & S. willmorei
\end{tabular}

Strains assigned to subcluster 1-4: Streptomyces olivaceus (6)

$\begin{array}{ccllll}1-4 & 1-4 & S . \text { flavogriseus } & \text { "DSM 40990 } & \text { ATCC } 3331 \\ 1-4 & 1-4 & \text { S. olivaceus } & \text { *DSM 41536 } & \text { ATCC } 11626 \\ 1-4 & 1-4 & \text { S. olivaceus } & \text { *DSM 41538 } & \text { ATCC 21379 } \\ 1-4 & 1-4 & \text { S. olivaceus } & \text { "DSM 41539 } & \text { ATCC 21381 } \\ 1-4 & 1-4 & \text { S. olivaceus } & \text { *DSM 41540 } & \text { ATCC 21382 } \\ 1-4 & 1-4 & S . \text { scabies } & \text { DSM 40611 } & \text { ATCC 3352 }\end{array}$

DSM $40856 \quad$ ATCC 3347

DSM 40078 ATCC 23962 ISP 5078

*DSM 40395(T) ATCC 25497 ISP 5395

*DSM 40255(T) ATCC 23963 ISP 5255

*DSM 40993

DSM 40920

DSM 40992

DSM 40969

DSM 40977

DSM 40615

DSM 40200

*DSM 40257

*DSM 41585

DSM 40201

*DSM 40459(T)

*DSM 40459

ATCC 15886 ISP 5200 ATCC 11861 ISP 5257 NRRL B-1792

ATCC 15895 ISP 5201

ATCC 6867 ISP 5459

* olivaceus (6)

*DSM 41536

* DSM 41539

DSM 40611

ATCC 3352

Strains assigned to subcluster 1-5: Streptomyces antibioticus (19)

\begin{tabular}{|c|c|c|c|}
\hline $1-5$ & S. achromogenes & ${ }^{*}$ DSM $40028^{(T)}$ & ATCC 12767 ISP 5028 \\
\hline $1-5$ & S. antibioticus & *DSM 40715 & \\
\hline $1-5$ & S. antibioticus & ${ }^{*}$ DSM 40725 & \\
\hline $1-5$ & S. antibioticus & *DSM 40868 & ATCC 11891 \\
\hline $1-5$ & S. antibioticus & *DSM 41566 & ATCC 14890 \\
\hline 9 & S. antibioticus & ${ }^{*}$ DSM 41567 & ATCC 15848 \\
\hline 9 & $S$. antibioticus & * DSM 41569 & ATCC 31771 \\
\hline 11 & S. argenteolus & ${ }^{*} \mathrm{DSM} 40226^{(\mathrm{T})}$ & ATCC 11009 ISP 5226 \\
\hline 11 & S. aurantiogriseus & ${ }^{*} \mathrm{DSM} 40138^{\mathrm{T}}$ & ATCC 19887 ISP 5138 \\
\hline 9 & S. eurythermus & ${ }^{*}$ DSM $40014^{(T)}$ & ATCC 14975 ISP 5014 \\
\hline 11 & S. galbus & *DSM 40480 & ATCC 14077 ISP 5480 \\
\hline 11 & S. griseochromogenes & ${ }^{*}$ DSM 40499(T) & ATCC 14511 ISP 549 \\
\hline $1 \cdot 16$ & S. griseoluteus & ${ }^{*} \mathrm{DSM} 40392^{\top}$ & ATCC 12768 ISP 53S \\
\hline $1-16$ & S. lucensis & ${ }^{*} \mathrm{DSM} 40317^{(\mathrm{T})}$ & ATCC 17804 ISP 531 \\
\hline 9 & S. nogalater & ${ }^{*} \mathrm{DSM} 40546^{\mathrm{T}}$ & ATCC 27451 ISP 5546 \\
\hline 59 & $S$. recifensis & ${ }^{*} \mathrm{DSM} 40115^{(\mathrm{T})}$ & ATCC 19803 ISP 5115 \\
\hline 9 & S. spadicis & DSM 40476 & ATCC 19017 ISP 5476 \\
\hline $1-16$ & S. violaceusniger & *DSM 41601 & NRRL B-5799 \\
\hline 9 & Sv. lavenduligriseum & ${ }^{*} \mathrm{DSM} 40487^{(\mathrm{T})}$ & ATCC 13306 ISP 5487 \\
\hline
\end{tabular}

Strains assigned to subcluster 1-6: Streptomyces flaveolus (16)

$\begin{array}{lll}1-6 & 1-31 & S \text {. alboniger }\end{array}$

1-6 1-14 $S$. collinus

1-6 1-10 S. echinatus

1-6 1-13 S. flaveolus

1-6 1-16 S. griseofuscus

1-6 $1-14 \quad S$. heimi

1-6 1-16 S. hygroscopicus

1-6 1-16 S. lavendulae

1-6 $\quad 1-16 \quad S$. misionensis

1-6 1-16 S. murinus

1-6 1-15 S. naganishii

1-6 $\quad 1-16 \quad S$. roseoluteus

1-6 1-14 Streptomyces sp.

1-6 1-16 S. umbrinus

1-6 1-12 S. umbrosus

1-6 1-16 S. violaceusniger

*DSM 40043(T) ATCC 12461 ISP 5043

*DSM 40733

*DSM 40013(T) ATCC 19748 ISP 5013

${ }^{*}$ DSM 40061 ${ }^{\mathrm{T}}$ ATCC 3319 ISP 5061

${ }^{*}$ DSM 40191(T) ATCC 23916 ISP 5191

DSM 40328 ATCC 25460 ISP 5328

*DSM 40771 ATCC 10976

*DSM 40708

*DSM 40306(T) ATCC 14991 ISP 5306

*DSM 40091(T) ATCC 19788 ISP 5091

*DSM 40282(T) ATCC 23939 ISP 5282

DSM 40240 ATCC 23975 ISP 5240

DSM 4078

*DSM 40278(T) ATCC 19929 ISP 5278

DSM 40242 ATCC 25504 ISP 5242

*DSM $40710 \quad$ NRRL 2834

Strains assigned to subcluster 1-7: Streptomyces diastatochromogenes (25)

1-7 $1-19$ S. actuosus

1-7 1-15 S. antibioticus \#

DSM 40337

ATCC 25421 ISP 5337

1-7 $1-15$ S. arenae

1-7 $1-19 \quad S$. aureus

*DSM 40234

*DSM 40734

DSM 40867

ATCC 8663 ISP 5234

ATCC 15437
Williams $\quad$ Bergey category

group 9 and species no. $\dagger^{\dagger}$

A 03

A 1B Category I Species no. 02

A 1B Category I Species no. 02

Category I Species no. 02

A 06 Category I Species no. 05 Category III Species no. 04

A 1B Category I Species no. 02 Category I Species no. 02

Category I Species no. 03 Category I Species no. 03 Category I Species no. 03 Category I Species no. 03 Category I Species no. 03

A 19 Category I Species no. 12 Category I Species no. 21 Category I Species no. 21 Category I Species no. 21 Category I Species no. 21 Category I Species no. 21 Category I Species no. 21 A 15 Category I Species no. 08

A Sm Category III Species no. 09

A 23 Category I Species no. 20 Category I Species no. 08

A 18 Category I Species no. 11

C 43 Category II Species no. 11

A 31 Category I Species no. 21

A 34 Category III Species no. 06

A 23 Category I Species no. 20

A 34

Category I Species no. 16

A 34 Streptov. Species no. 02

A 1B Category I Species no. 02

Category I Species no. 11

A 18 Category I Species no. 11

A 24 Category II Species no. 05

A 12 Category I Species no. 07

Category I Species no. 16 Category I Species no. 22

A 31 Category I Species no. 21

A 17 Category I Species no. 10

A 31 Category I Species no. 21

A 17

A 05 Category I Species no. 04

A 33

Category I Species no. 16

A 31 Category I Species no. 21 Category I Species no. 11 
Table 1 (continued)

\begin{tabular}{|c|c|c|c|c|c|c|c|}
\hline \multirow{2}{*}{$\frac{S_{\mathrm{SM}}{ }^{\dagger}}{1-7}$} & \multirow{2}{*}{$\frac{S_{\mathrm{J}} \ddagger}{1-15}$} & \multirow[t]{2}{*}{ Strain designation $\S$} & \multicolumn{2}{|c|}{ Strain numbers $\|$} & \multirow[t]{2}{*}{$\begin{array}{l}\text { Williams } \\
\text { group } \mathbb{T}\end{array}$} & \multicolumn{2}{|r|}{$\begin{array}{l}\text { Bergey category } \\
\text { and species no. }{ }^{\dagger}\end{array}$} \\
\hline & & & ${ }^{*}$ DSM $40056^{\mathrm{T}}$ & ATCC 3310 ISP 5056 & & Category IV & Species no. 02 (white series) \\
\hline $1-7$ & $1-15$ & S. capoamus & ${ }^{*}$ DSM $40494^{(n)}$ & ATCC 19006 ISP 5494 & C 45 & Category II & Species no. 13 \\
\hline $1-7$ & $1-15$ & $S$. diastatochromogenes & *DSM 40700 & & & Category I & Species no. 12 \\
\hline $1-7$ & $1-15$ & S. echinatus & *DSM 40730 & & & & \\
\hline $1-7$ & $1-19$ & S. fumanus & ${ }^{*}$ DSM $40154^{(T)}$ & ATCC 19904 ISP 5154 & A 18 & Category I & Species no. 11 \\
\hline $1-7$ & $1-15$ & S. galilaeus & ${ }^{*}$ DSM $40481^{(T)}$ & ATCC 14969 ISP 5481 & A 19 & Category I & Species no. 12 \\
\hline $1-7$ & $1-21$ & S. ghanaensis & ${ }^{*} \mathrm{DSM} 40746^{\mathrm{T}}$ & ATCC 14672 & & Category IV & Species no. 05 (green series) \\
\hline $1-7$ & $1-15$ & S. glaucescens & *DSM 40922 & & & Category III & I Species no. 05 \\
\hline $1-7$ & $1-15$ & S. glaucescens & *DSM 40923 & & & Category III & I Species no. 05 \\
\hline $1-7$ & $1-15$ & S. glaucescens & *DSM 40924 & & & Category III & I Species no. 05 \\
\hline $1-7$ & $1-15$ & S. griseoaurantiacus & ${ }^{*}$ DSM $40430^{(T)}$ & ATCC 19840 ISP 5430 & A 12 & Category I & Species no. 07 \\
\hline $1-7$ & $1-14$ & S. griseoflavus & *DSM 40698 & NRRL 2717 & & Category I & Species no. 17 \\
\hline $1-7$ & $1-19$ & S. griseosporeus & ${ }^{*}$ DSM 40562 & ATCC 27435 ISP 5562 & A 23 & Category I & Species no. 20 \\
\hline $1-7$ & $1-14$ & S. lavendulae & *DSM 40713 & NRRL 2856 & & Category I & Species no. 21 \\
\hline $1-7$ & $1-19$ & S. mirabilis & ${ }^{*} \mathrm{DSM} 40553^{(\mathrm{T})}$ & ATCC 27447 ISP 5553 & A 19 & Category I & Species no. 12 \\
\hline $1-7$ & $1-19$ & S. olivaceus & *DSM 41542 & ATCC 21549 & & Category I & Species no. 03 \\
\hline $1-7$ & $1-15$ & S. rishiriensis & ${ }^{*}$ DSM $40489^{(T)}$ & ATCC 14812 ISP 5489 & A 19 & Category I & Species no. 12 \\
\hline $1-7$ & $1-15$ & Streptomyces sp. & DSM 40981 & & & & \\
\hline $1-7$ & $1-19$ & Streptomyces sp. & DSM 40836 & & & & \\
\hline $1-7$ & $1-15$ & S. thermoflavus & DSM 40574 & ATCC 27473 ISP 5574 & A 36 & & \\
\hline $1-7$ & $1-15$ & S. versipellis & DSM 40491 & ATCC 27475 ISP 5491 & A Sm & & \\
\hline
\end{tabular}

Strain assigned to subcluster 1-8: Streptoverticillium luteoreticuli (1)

\section{1-8 1-17 Sv. luteoreticuli}

1-7 S. albocinerescens

1-7 S. avellaneus

1-7 S. candidus

38 S. cinereoruber

1-7 S. cremeus

1-7 S. exfoliatus \#

1-7 S. felleus

1-7 S. filamentosus

1-7 S. flavochromogenes

1-7 S. gardneri

1-7 S. griseofaciens

1-7 S. hydrogenans

1-7 S. litmocidini

1-7 S. narbonensis

1-7 S. nashvillensis

1-7 S. olivaceus

1-7 S. omiyaensis

1-7 S. roseofulvus

1-7 S. roseolus

1-7 S. roseosporus

1-7 S. roseus

1-7 S. scabies

1-7 Streptomyces sp.

1-7 S. spiroverticillatus

1-7 S. tanashiensis

1-7 S. venezuelae

1-7 S. venezuelae

1-7 S. violaceorectus

1-7 S. violascens

1-7 S. zaomyceticus

1-3 S. gelaticus

1-3 S. naraensis

1-3 S. niveus

$$
\begin{array}{rl}
1-23 & S . \text { diastaticus } \\
1-23 & \text { S. graminofaciens } \\
6 & \text { S. novaecaesareae } \\
1-23 & \text { S. olivaceus } \\
1-23 & \text { S. pristinaespiralis } \\
6 & \text { S. thermoviolaceus }
\end{array}
$$

DSM $40509 \quad$ ATCC 27446 ISP 5509

Strains assigned to cluster 2: Streptomyces exfoliatus (30)

DSM 40794 NRRL 3419

${ }^{*}$ DSM 40554(T) ATCC 23730 ISP 5554

DSM 40141 ATCC 19891 ISP 5141

${ }^{*}$ DSM 40012(T) ATCC 19740 ISP 5012

${ }^{*}$ DSM $40147^{(T)}$ ATCC 19897 ISP 5147

${ }^{*}$ DSM 40060 $\quad$ ATCC 12627 ISP 5060

*DSM 40976

${ }^{*}$ DSM 40022(T) ATCC 19753 ISP 5022

DSM 40541 ATCC 14841 ISP 5541

${ }^{*}$ DSM 40064(T) ATCC 9604 ISP 5064

DSM 40816 ATCC 13180

${ }^{*}$ DSM 40586(T) ATCC 19631 ISP 5586

*DSM 40164(T) ATCC 19914 ISP 5164

*DSM 40016(T) ATCC 19790 ISP 5016

*DSM 40314(T) ATCC 25476 ISP 5314

*DSM 40702

*DSM 40552(T) ATCC 27454 ISP 5552

${ }^{*}$ DSM 40172(T) ATCC 19921 ISP 5172

${ }^{*}$ DSM 40174(T) ATCC 23210 ISP 5174

${ }^{*}$ DSM 40122(T) ATCC 23958 ISP 5122

DSM 40076 ATCC 19808 ISP 5076

DSM 40996

DSM 40887

*DSM 40036(T) ATCC 19811 ISP 5036

${ }^{*}$ DSM 40195 $\quad$ ATCC 23967 ISP 5195

*DSM 40230(T) ATCC 10712 ISP 5230

*DSM 40634 ATCC 10595

*DSM 40279(T) ATCC 25514 ISP 5279

${ }^{*}$ DSM 40183(T) ATCC 23968 ISP 5183

${ }^{*}$ DSM 40196(T) ATCC 27482 ISP 5196

Strains assigned to cluster 3: Streptomyces gel

${ }^{*}$ DSM $40065^{\mathrm{T}}$

DSM 40508

ATCC 3323 ISP 5065

ATCC 13788 ISP 5508

* DSM 40613

A 03

A 05

A 1 B

A 05

A 05

A 05

A 04

A 05

A 05

A 04

A 05

A 05

A 14

A 05

A 05

A 07

A 06

A 06

A 05

A 06

A 05

Streptov. Species no. 07

Category II Species no. 17

Category I Species no. 04

Category I Species no. 02

Category I Species no. 04

Category I Species no. 01

Category I Species no. 04

Category I Species no. 04

Category I Species no. 04 Category I Species no. 04 Category I Species no. 04 Category I Species no. 04 Category I Species no. 03 Category I Species no. 04 Category II Species no. 04 Category I Species no. 04 Category I Species no. 04 Strains assigned to cluster 4: Streptomyces graminofaciens (6)

*DSM 40650

*DSM 40559

${ }^{*}$ DSM $40358^{\mathrm{T}}$

*DSM 41537

DSM 40338

*DSM 40443(T)
ATCC 12705 ISP 5559

ATCC 27452 ISP 5358

ATCC 12019

ATCC 25486 ISP 5338

ATCC 19283 ISP 5443
A Sm

A $1 \mathrm{C}$

A 26

J $\mathrm{Sm}$

A 26

C 45
Category I Species no. 05 Category IV Species no. 30 (grey series)

Category I Species no. 05

Category I Species no. 05

Category I Species no. 04

Category I Species no. 05

Category I Species no. 04

Category III Species no. 11

Category I Species no. 02

Category I Species no. 12

Category III Species no. 03

Category III Species no. 25

Category I Species no. 03

Category II Species no. 13 
Table 1 (continued)

\begin{tabular}{|c|c|c|c|c|c|}
\hline$S_{\mathrm{SM}} \dagger$ & $S_{\mathrm{J}} \ddagger$ & Strain designation§ & Strain numbers\| & $\begin{array}{l}\text { Williams } \\
\text { group }\end{array}$ & $\begin{array}{c}\text { Bergey category } \\
\text { and species no. }{ }^{\dagger}\end{array}$ \\
\hline
\end{tabular}

$\begin{array}{lll}5 & 10 & \text { S. longisporoflavus } \\ 5 & 29 & \text { S. michiganensis } \\ 5 & 29 & \text { S. parvus } \\ 5 & 29 & \text { S. puniceus } \\ 5 & 29 & \text { S. xanthochromogenes }\end{array}$

\begin{tabular}{|c|c|c|}
\hline 6 & $1-10$ & S. albaduncus \\
\hline 6 & 10 & S. albogriseolus \\
\hline 6 & $1-18$ & S. albogriseolus \\
\hline 6 & $1-18$ & S. althioticus \\
\hline 6 & $1-18$ & S. ambofaciens \\
\hline 6 & $1-18$ & S. ambofaciens \\
\hline 6 & $1-18$ & S. arabicus \\
\hline 6 & 10 & S. atroolivaceus \\
\hline 6 & $1-18$ & S. aureofaciens \\
\hline 6 & $1-2$ & S. baarnensis \\
\hline 6 & $1-18$ & S. caesius \\
\hline 6 & $1-18$ & S. calvus \\
\hline 6 & $1-18$ & S. carnosus \\
\hline 6 & $1-18$ & S. cellulosae \\
\hline 6 & $1-18$ & S. chromofuscus \# \\
\hline 6 & $1-18$ & $\begin{array}{l}S . \text { cinereoruber subsp. } \\
\text { fructofermentans }\end{array}$ \\
\hline 6 & $1-18$ & S. coelescens \\
\hline 6 & $1-18$ & S. cyanocolor \\
\hline 6 & $1-18$ & S. cyanocolor \\
\hline 6 & $1-18$ & S. cyanogenus \\
\hline 6 & 10 & S. daghestonicus \\
\hline 6 & $1-18$ & S. diastaticus \\
\hline 6 & $1-18$ & S. fimbriatus \\
\hline 6 & $1-10$ & S. flavoviridis \\
\hline 6 & $1-18$ & $S$. fradiae \\
\hline 6 & $1-18$ & S. fradiae \\
\hline 6 & $1-10$ & S. galbus \\
\hline 6 & $1-18$ & S. gancidicus \\
\hline 6 & $1-18$ & S. geysiriensis \\
\hline 6 & $1-10$ & S. glaucescens \\
\hline 6 & $1-18$ & S. griseoflavus \\
\hline 6 & $1-18$ & S. griseoflavus \\
\hline 6 & $1-18$ & S. griseoincarnatus \\
\hline 6 & $1-10$ & S. griseomycini \\
\hline 6 & 10 & S. griseoroseus \\
\hline 6 & $1-18$ & S. griseorubens \\
\hline 6 & $1-10$ & S. griseostramineus \\
\hline 6 & 10 & S. griseoviridis \# \\
\hline 6 & $1-18$ & S. lividans \\
\hline 6 & $1-18$ & S. lusitanus \\
\hline 6 & 10 & S. macrosporeus \\
\hline 6 & $1-18$ & S. malachitofuscus \\
\hline 6 & $1-18$ & S. malachitorectus \\
\hline 6 & $1-18$ & S. matensis \\
\hline 6 & $1-18$ & S. minutiscleroticus \\
\hline 6 & $1-18$ & S. mutabilis \\
\hline 6 & $1-11$ & S. nodosus \\
\hline 6 & $1-18$ & S. parvulus \\
\hline 6 & $1-18$ & S. parvulus \\
\hline 6 & $1-10$ & S. pilosus \\
\hline 6 & $1-18$ & S. pilosus \\
\hline 6 & $1-18$ & S. plicatus \\
\hline 6 & $1-18$ & S. pseudogriseolus \\
\hline 6 & $1-18$ & S. pulcher \\
\hline 6 & $1-18$ & S. rochei\# \\
\hline 6 & 10 & S. rosa \\
\hline 6 & $1-2$ & S. rubiginosohelvolus \\
\hline 6 & $1-18$ & S. rubiginosus \\
\hline
\end{tabular}

Strains assigned to cluster 5: Streptomyces parvus (5)

*DSM 40165 $\quad$ ATCC 19915 ISP 5165 A 39 Category II Species no. 10

*DSM 40015(T) ATCC 14970 ISP 5015 A 06 Category I Species no. 05

*DSM 40829 ATCC $12320 \quad$ Category I Species no. 02

*DSM 40083(T) ATCC 19801 ISP 5083 A 09 Category II Species no. 02

${ }^{*}$ DSM 40111 $\quad$ ATCC 19818 ISP 5111 F 63 Category II Species no. 15

Strains assigned to cluster 6: Streptomyces rochei (79)

${ }^{*}$ DSM 40478 $\quad$ ATCC 14698 ISP 5478

*DSM 40712 NRRL 2835

*DSM 40003(T) ATCC 23875 ISP 5003

*DSM 40092(T) ATCC 19724 ISP 5092

*DSM $40697 \quad$ NRRL 2531

*DSM 40053(T) ATCC 23877 ISP 5053

*DSM 40252(T) ATCC 23881 ISP 5225

*DSM 40137 $\quad$ ATCC 19725 ISP 5137

*DSM 40731

*DSM 40232(T) ATCC 23885 ISP 5232

DSM 40419 ATCC 19828 ISP 5419

${ }^{*}$ DSM 40010(T) ATCC 13382 ISP 5010

DSM 40294 ATCC 25437 ISP 5294

${ }^{*}$ DSM 40362 $\quad$ ATCC 25439 ISP 5362

${ }^{*}$ DSM $40273^{\mathrm{T}} \quad$ ATCC 23896 ISP 5273

A 12

Category IV Species no. 02 (grey series)

Category I Species no. 07

$\begin{array}{lll}\text { A } 12 & \text { Category I } & \text { Species no. 07 } \\ \text { A } 12 & \text { Category I } & \text { Species no. 07 }\end{array}$

Category I Species no. 20

A 23 Category I Species no. 20

A 12 Category I Species no. 07

A 03 Category II Species no. 20

Category II Species no. 04

A 1B Category I Species no. 02

A 21

DSM 40692(T) NRRL 2588

*DSM 40421(T) ATCC 19830 ISP 5421

DSM 40245

DSM 40425

DSM 40426

ATCC 19835 ISP 5425

ATCC 19836 ISP 5426

*DSM 40149(T) ATCC 23620 ISP 5149

*DSM 40934

${ }^{*}$ DSM 40942 ${ }^{\mathrm{T}} \quad$ ATCC 15051

DSM 40153 ATCC 19903 ISP 5153

*DSM 40696

NRRL 2598

*DSM 40711 NRRL 2765

*DSM 40089(T) ATCC 23910 ISP 5089

${ }^{*}$ DSM 40935T NRRL B-1872

*DSM 40742 $\quad$ ATCC 15303

*DSM 40155T ATCC 23622 ISP 5155

*DSM 40456 ${ }^{\mathrm{T}} \quad$ ATCC 25456 ISP 5456

*DSM 40709 NRRL 2833

*DSM 40274(T) ATCC 23623 ISP 5274

*DSM 40159(T) ATCC 23625 ISP 5159

DSM 40768 ATCC 12125

${ }^{*}$ DSM 40160(T) ATCC 19909 ISP 5160

*DSM 40161 ${ }^{\mathrm{T}}$ ATCC 23628 ISP 5161

${ }^{*}$ DSM 40229r ATCC 23920 ISP 5229

DSM 40434 ATCC 19844 ISP 5434

*DSM 40568(T) ATCC 15842 ISP 5568

DSM 40096 ATCC 19783 ISP 5096

DSM 40332 ATCC 25471 ISP 5332

DSM 40333 ATCC 25472 ISP 5333

*DSM 40188(T) ATCC 23935 ISP 5188

DSM 40301(T) ATCC 17757 ISP 5301

*DSM 40169(T) ATCC 19919 ISP 5169

*DSM 40109(T) ATCC 14899 ISP 5109

*DSM 40728

*DSM 40048(T) ATCC 12434 ISP 5048

*DSM 40097(T) ATCC 19797 ISP 5097

*DSM 40714 NRRL 2857

*DSM 40319(T) ATCC 25483 ISP 5319

*DSM 40026(T) ATCC 12770 ISP 5026

DSM 40566 ATCC 13849 ISP 5566

${ }^{*}$ DSM 40231 ${ }^{\mathrm{T}} \quad$ ATCC 10739 ISP 5231

DSM 40533 ATCC 27462 ISP 5533

*DSM 40176 $\quad$ ATCC 19926 ISP 5176

*DSM 40177(T) ATCC 19927 ISP 5177
A 12

A 15

A 13

A 15

Category I Species no. 07

Category I Species no. 08

Category I Species no. 04

A 21

A 21

A 21

A 17 Category I Species no. 10 Category I Species no. 12

Category IV Species no. 16 (grey series)

A 28

Category II Species no. 18

Category II Species no. 18

A 15 Category I Species no. 08

Category IV Species no. 17 (grey series)

Category IV Species no. 18 (grey series)

A 28

A 37

Category III Species no. 05

Category I Species no. 17

Category I Species no. 17

A 13 Category II Species no. 03

A 12 Category I Species no. 07

A 12 Category I Species no. 07

F 60 Category IV Species no. 06 (green series)

A 17 Category I Species no. 10

A 21

C 44

A 38

Category II Species no. 12

A 12

A 15

A 12

A 35

A 12

A 37

A 12

A 12

A 12

A 12

A 17

A 12
Category I Species no. 07

Category I Species no. 08

Category I Species no. 07

Category II Species no. 08

Category I Species no. 07

Category I Species no. 17

Category I Species no. 17

Category I Species no. 07

Category I Species no. 07

Category I Species no. 07

Category IV Species no. 12 (red series)

Category I Species no. 07
Category II Species no. 03 
Table 1 (continued)

\begin{tabular}{|c|c|c|c|c|c|c|c|}
\hline$S_{\mathrm{SM}}^{\dagger}$ & $S_{\mathrm{J}} \ddagger$ & Strain designation $\S$ & \multicolumn{2}{|c|}{ Strain numbers $\|$} & \multicolumn{2}{|l|}{$\begin{array}{l}\text { Williams } \\
\text { groupף }\end{array}$} & $\begin{array}{l}\text { Bergey category } \\
\text { and species no. } \dagger \dagger\end{array}$ \\
\hline 6 & $1-18$ & S. scabies & DSM 40960 & & & & \\
\hline 6 & $1-18$ & Streptomyces sp. & DSM 40916 & & & & \\
\hline 6 & $1-18$ & Streptomyces sp. & DSM 40975 & & & & \\
\hline 6 & $1-18$ & Streptomyces sp. (lazureus) & DSM 40433 & ATCC 19843 ISP 5433 & & & \\
\hline 6 & $1-18$ & Streptomyces sp. (mitakaensis) & DSM 40972 & & & & \\
\hline 6 & $1-18$ & Streptomyces sp. (mitakaensis) & DSM 40973 & & & & \\
\hline 6 & $1-18$ & S. tendae & ${ }^{*} \mathrm{DSM} 40101^{(\mathrm{T})}$ & ATCC 19812 ISP 5101 & A 12 & Category I & Species no. 07 \\
\hline 6 & $1-18$ & S. tendae & *DSM 40729 & & & Category I & Species no. 07 \\
\hline 6 & $1-18$ & S. thermodiastaticus & ${ }^{*} \mathrm{DSM} 40573^{(\mathrm{T})}$ & ATCC 27472 ISP 5573 & A $1 C$ & Category I & Species no. 03 \\
\hline 6 & $1-18$ & S. thermophilus & DSM 40365 & ATCC 19282 ISP 5365 & A 15 & & \\
\hline 6 & $1-18$ & S. tuirus & *DSM 40505 & & A 21 & Category I & Species no. 14 \\
\hline 6 & $1-18$ & S. variabilis & ${ }^{*}$ DSM 40179(T) & ATCC 19930 ISP 5179 & A 12 & Category I & Species no. 07 \\
\hline 6 & $1-18$ & S. vinaceusdrappus & ${ }^{*} \mathrm{DSM} 40470^{(\mathrm{T})}$ & ATCC 25511 ISP 5470 & A 12 & Category I & Species no. 07 \\
\hline 6 & $1-18$ & S. violaceolatus & ${ }^{*}$ DSM $40438^{(T)}$ & ATCC 19847 ISP 5438 & A 21 & Category I & Species no. 14 \\
\hline 6 & $1-18$ & S. violaceoruber & *DSM 40687 & & & Category IV & Species no. 34 (grey series) \\
\hline 6 & $1-18$ & S. violaceoruber & * DSM 40783 & & & Category IV & Species no. 34 (grey series) \\
\hline 6 & $1-18$ & S. viridiviolaceus & ${ }^{*}$ DSM $40280^{T}$ & ATCC 27478 ISP 5280 & & Category IV & Species no. 35 (grey series) \\
\hline 6 & $1-18$ & S. viridodiastaticus & ${ }^{*}$ DSM $40249^{\top}$ & ATCC 25518 ISP 5249 & & Category IV & Species no. 36 (grey series) \\
\hline 6 & $1-4$ & S. viridogenes & DSM 40454 & ATCC 3372 ISP 5454 & A 03 & & \\
\hline 6 & $1-18$ & S. viridosporus & ${ }^{*}$ DSM $40243^{(T)}$ & ATCC 27479 ISP 5243 & A 15 & Category I & Species no. 08 \\
\hline 6 & $1-18$ & S. werraensis & ${ }^{*}$ DSM $40486^{(T)}$ & ATCC 14424 ISP 5486 & A 12 & Category I & Species no. 07 \\
\hline \multicolumn{8}{|c|}{ Strains assigned to cluster $7:$ Streptomyces prasinopilosus (7) } \\
\hline 7 & 3 & S. albocyaneus & DSM 40197 & ATCC 15845 ISP 5197 & A Sm & & \\
\hline 7 & 3 & S. cellostaticus & *DSM 40189(T) & ATCC 23894 ISP 5189 & A 06 & Category I & Species no. 05 \\
\hline 7 & 3 & S. cyanoalbus & ${ }^{*} \mathrm{DSM} 40198^{(\mathrm{T})}$ & ATCC 15859 ISP 5198 & A 37 & Category I & Species no. 17 \\
\hline 7 & 3 & S. prasinopilosus & ${ }^{*}$ DSM 40098(T) & ATCC 19799 ISP 5098 & A 37 & Category I & Species no. 17 \\
\hline 7 & 3 & S. prasinus & ${ }^{*} \mathrm{DSM} 40099(\mathrm{~T})$ & ATCC 19800 ISP 5099 & A 37 & Category I & Species no. 17 \\
\hline 7 & 3 & $S$. prasinus & *DSM 40688 & & & Category I & Species no. 17 \\
\hline 7 & 3 & Streptomyces sp. & DSM 40985 & & & & \\
\hline \multicolumn{8}{|c|}{ Strains assigned to cluster $8:$ Streptomyces sp. (4) } \\
\hline 8 & 20 & S. hygroscopicus & ${ }^{*}$ DSM 41531 & ATCC 31039 & & Category I & Species no. 16 \\
\hline 8 & 19 & S. scabies & DSM 40999 & & & & \\
\hline 8 & 19 & S. scabies & DSM 40995 & & & & \\
\hline 8 & 19 & Streptomyces sp. & DSM 40750 & ATCC 21619 & & & \\
\hline \multicolumn{8}{|c|}{ Strains assigned to cluster 9: Streptomyces violaceus (118) } \\
\hline 9 & $1-19$ & S. afghaniensis & ${ }^{*}$ DSM $40228(\mathrm{~T})$ & ATCC 23871 ISP 5228 & A 18 & Category I & Species no. 11 \\
\hline 9 & $1-19$ & S. alanosinicus & ${ }^{*}$ DSM $40606^{T}$ & ATCC 15710 ISP 5606 & & Category IV & Species no. 01 (grey series) \\
\hline 9 & $1-19$ & S. antibioticus & - DSM 40723 & NRRL 3207 & & Category I & Species no. 21 \\
\hline 9 & $1-19$ & $S$. arenae & *DSM 40737 & & & Category I & Species no. 11 \\
\hline 9 & $1-19$ & S. arenae & ${ }^{*}$ DSM $40293^{(\mathrm{T})}$ & ATCC 25428 ISP 5293 & A 18 & Category I & Species no. 11 \\
\hline 9 & $1-9$ & S. atrofaciens & DSM 40475 & ATCC 27418 ISP 5475 & A 33 & & \\
\hline 9 & $1-19$ & S. azureus & ${ }^{*}$ DSM $40106^{(T)}$ & ATCC 14921 ISP 5106 & A 18 & Category I & Species no. 11 \\
\hline 9 & $1-19$ & S. bicolor & DSM 40140 & ATCC 23614 ISP 5140 & A 18 & & \\
\hline 9 & $1-19$ & S. bottropensis & ${ }^{*} \mathrm{DSM} 40262^{(\mathrm{T})}$ & ATCC 25435 ISP 5262 & A 19 & Category I & Species no. 12 \\
\hline 9 & $1-19$ & $S$. caelestis & *DSM 40084(T) & ATCC 15084 ISP 5084 & A 18 & Category I & Species no. 11 \\
\hline 9 & $1-19$ & S. canarius & - DSM 40528(T) & ATCC 27423 ISP 5528 & A 20 & Category I & Species no. 13 \\
\hline 9 & $1-19$ & S. canus & ${ }^{*} \mathrm{DSM} 40017^{\mathrm{T}}$ & ATCC 12237 ISP 5017 & A 25 & Category III & I Species no. 02 \\
\hline 9 & $1-19$ & S. chartreusis & ${ }^{*} \mathrm{DSM} 40085^{(\mathrm{T})}$ & ATCC 14922 ISP 5085 & A 18 & Category I & Species no. 11 \\
\hline 9 & $1-19$ & S. chartreusis & *DSM 41592 & NRRL B-2199 & & Category I & Species no. 11 \\
\hline 9 & $1-19$ & S. chartreusis & *DSM 41593 & NRRL B-8150 & & Category I & Species no. 11 \\
\hline 9 & $1-19$ & S. chartreusis & * DSM 41594 & NRRL B-8151 & & Category I & Species no. 11 \\
\hline 9 & $1-19$ & S. chartreusis & *DSM 41595 & NRRL B-8152 & & Category I & Species no. 11 \\
\hline 9 & $1-19$ & S. chartreusis & *DSM 41596 & ATCC 21901 & & Category I & Species no. 11 \\
\hline 9 & $1-19$ & S. chartreusis & *DSM 41597 & NRRL B-16268 & & Category I & Species no. 11 \\
\hline 9 & $1-19$ & S. chibaensis & *DSM $40220^{(\mathrm{T})}$ & ATCC 23895 ISP 5220 & A 24 & Category II & Species no. 05 \\
\hline 9 & $1-19$ & S. cinnabarinus & ${ }^{*} \mathrm{DSM} 40467^{(\mathrm{T})}$ & ATCC 23617 ISP 5467 & A 18 & Category I & Species no. 11 \\
\hline 9 & $1-19$ & S. coeliatus & DSM 40422 & ATCC 19833 ISP 5422 & & & \\
\hline 9 & $1-19$ & S. coerulatus & DSM 40424 & ATCC 19834 ISP 5424 & & & \\
\hline 9 & $1-19$ & S. coeruleofuscus & ${ }^{*}$ DSM $40144^{(T)}$ & ATCC 23618 ISP 5144 & A 18 & Category I & Species no. 11 \\
\hline 9 & $1-19$ & S. coeruleorubidus & ${ }^{*}$ DSM $40145^{(\mathrm{T})}$ & ATCC 13740 ISP 5145 & A 18 & Category I & Species no. 11 \\
\hline 9 & $1-19$ & S. coerulescens & ${ }^{*}$ DSM $40146(\mathrm{~T})$ & ATCC 19896 ISP 5146 & A 18 & Category I & Species no. 11 \\
\hline 9 & $1-19$ & S. collinus & ${ }^{*}$ DSM $40129^{(T)}$ & ATCC 19743 ISP 5129 & A 18 & Category I & Species no. 11 \\
\hline 9 & $1-19$ & S. collinus & *DSM 40726 & & & Category I & Species no. 11 \\
\hline 9 & $1-19$ & $S$. coralus & DSM $40256^{(T)}$ & ATCC 23901 ISP 5256 & A 19 & & \\
\hline
\end{tabular}


Table 1 (continued)

\begin{tabular}{|c|c|c|c|c|c|c|c|}
\hline \multirow{2}{*}{$\frac{S_{\mathrm{SM}} \dagger}{9}$} & \multirow{2}{*}{$\frac{S_{\mathrm{J}} \ddagger}{1-19}$} & \multirow[t]{2}{*}{ Strain designation $\S$} & \multicolumn{2}{|c|}{ Strain numbers\| } & \multirow{3}{*}{$\begin{array}{l}\begin{array}{l}\text { Williams } \\
\text { group } \mathbb{1}\end{array} \\
\text { A } 20\end{array}$} & \multirow{2}{*}{\multicolumn{2}{|c|}{$\begin{array}{cc}\begin{array}{c}\text { Bergey category } \\
\text { and species no.t† }\end{array} \\
\text { Category I } \quad \text { Species no. } 13\end{array}$}} \\
\hline & & & ${ }^{*}$ DSM $40340^{(T)}$ & ATCC 25444 ISP 5340 & & & \\
\hline 9 & $1-9$ & S. crystallinus & *DSM 40945 & & & Category IV & Species no. 03 (red series) \\
\hline 9 & 19 & S. curacoi & DSM 40107 & ATCC 13385 ISP 5107 & A 18 & Category I & Species no. 11 \\
\hline 9 & $1-19$ & S. cyaneus\# & ${ }^{*} \mathrm{DSM} 40108^{\mathrm{T}}$ & ATCC 14923 ISP 5108 & A 18 & Category I & Species no. 11 \\
\hline 9 & $1-19$ & S. diastatochromogenes & ${ }^{*}$ DSM $40449^{(\mathrm{T})}$ & ATCC 12309 ISP 5449 & A 19 & Category I & Species no. 12 \\
\hline 9 & $1-19$ & S. diastatochromogenes & *DSM 40608 & ATCC 23862 & & Category I & Species no. 12 \\
\hline 9 & $1-19$ & S. durhamensis & ${ }^{*}$ DSM 40539(T) & ATCC 23194 ISP 5539 & A 30 & Category II & Species no. 06 \\
\hline 9 & $1-19$ & S. filipinensis & ${ }^{*} \mathrm{DSM} 40112^{\mathrm{T}}$ & ATCC 23905 ISP 5112 & A 30 & Category II & Species no. 06 \\
\hline 9 & $1-19$ & S. flaveolus & *DSM 40719 & & & Category II & Species no. 05 \\
\hline 9 & $1-19$ & S. flavochromogenes & DSM 40651 & & & & \\
\hline 9 & $1-19$ & $S$. fradiae & *DSM 41544 & ATCC 15861 & & Category II & Species no. 18 \\
\hline 9 & $1-19$ & $S$. griseoruber & ${ }^{*} \mathrm{DSM} 40275^{(\mathrm{T})}$ & ATCC 23626 ISP 5275 & A 21 & Category I & Species no. 14 \\
\hline 9 & $1-19$ & S. griseorubiginosus & ${ }^{*} \mathrm{DSM} 40469^{(\mathrm{T})}$ & ATCC 23627 ISP 5469 & A 18 & Category I & Species no. 11 \\
\hline 9 & $1-19$ & S. hawaiiensis & - DSM $40042^{(\mathrm{T})}$ & ATCC 12236 ISP 5042 & A 18 & Category I & Species no. 11 \\
\hline 9 & $1-19$ & S. humidus & ${ }^{*} \mathrm{DSM} 40263^{(\mathrm{T})}$ & ATCC 12760 ISP 5263 & A 19 & Category I & Species no. 12 \\
\hline 9 & $1-19$ & S. hygroscopicus subsp. & & & & & \\
\hline & & ossamyceticus & DSM 40824(T) & ATCC 15420 & & Category I & Species no. 16 \\
\hline 9 & $1-19$ & S. iakyrus & •DSM 40482 & ATCC 15375 ISP 5482 & A 18 & Category I & Species no. 11 \\
\hline 9 & $1-19$ & S. indigocolor & DSM 40432 & ATCC 19842 ISP 5432 & A 18 & & \\
\hline 9 & $1-19$ & S. ipomoeae & *DSM 40818 & ATCC 11747 & & Category IV & Species no. 02 (blue series) \\
\hline 9 & $1-19$ & S. janthinus & ${ }^{*}$ DSM $40206^{(T)}$ & ATCC 15870 ISP 5206 & A 18 & Category I & Species no. 11 \\
\hline 9 & 20 & S. lavendulae & *DSM 41580 & ATCC 21138 & & Category I & Species no. 22 \\
\hline 9 & $1-19$ & S. lavendulae & *DSM 41577 & ATCC 15873 & & Category I & Species no. 22 \\
\hline 9 & $1-19$ & S. lavendulae & * DSM 41579 & ATCC 19008 & & Category I & Species no. 22 \\
\hline 9 & $1-19$ & S. lincolnensis & ${ }^{*} \mathrm{DSM} 40355^{(\mathrm{T})}$ & ATCC 25466 ISP 5355 & A 19 & Category I & Species no. 12 \\
\hline 9 & $1-19$ & S. lomondensis & *DSM 41428 & & & Category IV & Species no. 03 (blue series) \\
\hline 9 & $1-19$ & $S$. longisporus & ${ }^{*}$ DSM $40166^{(\mathrm{r})}$ & ATCC 23931 ISP 5166 & A 18 & Category I & Species no. 11 \\
\hline 9 & $1-19$ & S. longisporus & *DSM 40910 & & & Category I & Species no. 11 \\
\hline 9 & $1-19$ & $S$. luteogriseus & ${ }^{*}$ DSM $40483^{(T)}$ & ATCC 15072 ISP 5483 & A 18 & Category I & Species no. 11 \\
\hline 9 & $1-9$ & S. melanogenes & DSM 40192 & ATCC 23937 ISP 5192 & A 33 & Category II & Species no. 07 \\
\hline 9 & $1-19$ & $S$. minoensis & DSM 40031 & ATCC 19787 ISP 5031 & A 19 & & \\
\hline 9 & $1-19$ & S. neyagawaensis & ${ }^{*}$ DSM 40588(T) & ATCC 27449 ISP 5588 & A 18 & Category I & Species no. 11 \\
\hline 9 & $1-9$ & S. noboritoensis & ${ }^{*}$ DSM $40223^{T}$ & ATCC 25477 ISP 5223 & A 33 & Category II & Species no. 07 \\
\hline 9 & $1-19$ & S. olivaceoviridis & ${ }^{*}$ DSM $40334^{\top}$ & ATCC 23630 ISP 5334 & A 20 & Category I & Species no. 13 \\
\hline 9 & $1-19$ & S. olivochromogenes & ${ }^{*}$ DSM $40451^{(T)}$ & ATCC 3336 ISP 5451 & A 19 & Category I & Species no. 12 \\
\hline 9 & $1-19$ & S. olivochromogenes subsp. & & & & & \\
\hline & & cytovirinus & DSM 40828 & ATCC 12791 & & Category I & Species no. 12 \\
\hline 9 & $1-19$ & S. peruviensis & DSM 40592 & ATCC 27459 ISP 5592 & A 18 & & \\
\hline 9 & 19 & S. phaeochromogenes & ${ }^{*}$ DSM $40073^{\mathrm{T}}$ & ATCC 3338 ISP 5073 & A 40 & Category I & Species no. 18 \\
\hline 9 & $1-19$ & S. phaeopurpureus & ${ }^{*}$ DSM $40125^{(T)}$ & ATCC 23946 ISP 5125 & A 09 & Category II & Species no. 02 \\
\hline 9 & $1-19$ & S. phaeoviridis & - DSM $40285^{(\mathrm{T})}$ & ATCC 23947 ISP 5285 & A 19 & Category I & Species no. 12 \\
\hline 9 & $1-19$ & S. pseudovenezuelae & ${ }^{*}$ DSM $40212^{(\mathrm{T})}$ & ATCC 23951 ISP 5212 & A 18 & Category I & Species no. 11 \\
\hline 9 & $1-19$ & S. purpurascens & ${ }^{*}$ DSM $40310^{(\mathrm{T})}$ & ATCC 25489 ISP 5310 & A 18 & Category I & Species no. 11 \\
\hline 9 & $1-19$ & S. purpurascens & *DSM 40747 & NRRL B-1480 & & Category I & Species no. 11 \\
\hline 9 & $1-19$ & $S$. regalis & DSM 40532 & ATCC 27460 ISP 5532 & & & \\
\hline 9 & $1-19$ & $S$. regensis & ${ }^{*}$ DSM $40551^{(T)}$ & ATCC 27461 ISP 5551 & A 20 & Category I & Species no. 13 \\
\hline 9 & $1-19$ & S. resistomycificus & ${ }^{*}$ DSM $40133^{(T)}$ & ATCC 19804 ISP 5133 & A 18 & Category I & Species no. 11 \\
\hline 9 & $1-19$ & $S$. roseogriseus & DSM 40488 & ATCC 12414 ISP 5488 & & & \\
\hline 9 & $1-19$ & $S$. roseoviolaceus & ${ }^{*} \mathrm{DSM} 40277^{(\mathrm{T})}$ & ATCC 25493 ISP 5277 & A 18 & Category I & Species no. 11 \\
\hline 9 & $1-19$ & S. scabies & DSM 40997 & & & & \\
\hline 9 & $1-19$ & S. scabies & DSM 41000 & & & & \\
\hline 9 & $1-19$ & S. scabies & DSM 40998 & & & & \\
\hline 9 & $1-19$ & Streptomyces sp. & DSM 40718 & & & & \\
\hline 9 & $1-19$ & Streptomyces sp. & DSM 40970 & & & & \\
\hline 9 & $1-19$ & Streptomyces sp.(gannmycicus) & DSM 40572 & ATCC 27434 ISP 5572 & A 30 & & \\
\hline 9 & $1-19$ & S. steffisburgensis & DSM 40547 & ATCC 27466 ISP 5547 & A 38 & & \\
\hline 9 & $1-19$ & S. tendae & * DSM 40732 & & & Category I & Species no. 07 \\
\hline 9 & $1-19$ & $S$. thermotolerans & DSM 40227 & ATCC 11416 ISP 5227 & A 18 & & \\
\hline 9 & $1-19$ & S. torulosus & ${ }^{*} \mathrm{DSM} 40894^{\mathrm{T}}$ & NRRL B-3889 & & Category IV & Species no. 31 (grey series) \\
\hline 9 & $1-19$ & S. violaceochromogenes & ${ }^{*}$ DSM $40181^{\mathrm{T}}$ & ATCC 19932 ISP 5181 & & Category IV & Species no. 33 (grey series) \\
\hline 9 & 19 & S. violaceus\# & ${ }^{*} \mathrm{DSM} 40082^{\mathrm{T}}$ & ATCC 15888 ISP 5082 & A 06 & Category I & Species no. 05 \\
\hline 9 & $1-19$ & S. violaceus & *DSM 40753 & & & Category I & Species no. 05 \\
\hline 9 & 20 & S. violaceusniger & *DSM 40182 & & & Category I & Species no. 16 \\
\hline 9 & $1-19$ & S. violarus & ${ }^{*} \mathrm{DSM} 40205^{(\mathrm{T})}$ & ATCC 15891 ISP 5205 & A 18 & Category I & Species no. 11 \\
\hline 9 & $1-19$ & S. violochromogenes & DSM 40207 & ATCC 15893 ISP 5207 & A 18 & & \\
\hline 9 & $1-19$ & S. viridis & DSM 40381 & ATCC 15732 ISP 5381 & A 27 & & \\
\hline
\end{tabular}


Table 1 (continued)

\begin{tabular}{|c|c|c|c|c|c|c|}
\hline$S_{\mathrm{SM}}^{\dagger}$ & $S_{\mathrm{J}} \ddagger$ & Strain designation§ & \multicolumn{2}{|c|}{ Strain numbers $\|$} & $\begin{array}{l}\text { Williams } \\
\text { group }\end{array}$ & $\begin{array}{l}\text { Bergey category } \\
\text { and species no. }{ }^{\dagger}\end{array}$ \\
\hline 9 & 9 & S. viridochromogenes & ${ }^{*}$ DSM 41586 & NRRL B-2310 & & Category III Species no. 04 \\
\hline 9 & 20 & S. viridochromogenes & ${ }^{*}$ DSM 41521 & ATCC 13759 & & Category III Species no. 04 \\
\hline 9 & 20 & $S$. viridochromogenes & ${ }^{*}$ DSM 41584 & NRRL B-1656 & & Category III Species no. 04 \\
\hline 9 & 20 & S. viridochromogenes & - DSM 41588 & NRRL B-3063 & & Category III Species no. 04 \\
\hline 9 & $1-19$ & S. viridochromogenes & ${ }^{*} \mathrm{DSM} 40110^{\mathrm{T}}$ & ATCC 14920 ISP 5110 & A 27 & Category III Species no. 04 \\
\hline 9 & $1-19$ & S. viridochromogenes & *DSM 40666 & & & Category III Species no. 04 \\
\hline 9 & $1-19$ & S. viridochromogenes & ${ }^{*}$ DSM 40736 & & & Category III Species no. 04 \\
\hline 9 & $1-19$ & $S$. viridochromogenes & ${ }^{*}$ DSM 40721 & NRRL B-2860 & & Category III Species no. 04 \\
\hline 9 & $1-19$ & $S$. viridochromogenes & *DSM 40784 & & & Category III Species no. 04 \\
\hline 9 & $1-19$ & S. viridochromogenes & ${ }^{*}$ DSM $40110 \mathrm{~d}^{(\mathrm{T})}$ & & & Category III Species no. 04 \\
\hline 9 & $1-19$ & S. viridochromogenes & *DSM 41514 & & & Category III Species no. 04 \\
\hline 9 & $1-19$ & S. viridochromogenes & *DSM 41516 & & & Category III Species no. 04 \\
\hline 9 & $1-19$ & S. viridochromogenes & *DSM 41520 & & & Category III Species no. 04 \\
\hline 9 & $1-19$ & S. viridochromogenes & ${ }^{*}$ DSM 40666d & & & Category III Species no. 04 \\
\hline 9 & $1-19$ & S. viridochromogenes & ${ }^{*}$ DSM 40513 & ATCC 3356 & & Category III Species no. 04 \\
\hline 9 & $1-19$ & S. viridochromogenes & * DSM 41517 & & & Category III Species no. 04 \\
\hline 9 & $1-19$ & S. viridochromogenes & ${ }^{*}$ DSM 41518 & & & Category III Species no. 04 \\
\hline 9 & $1-19$ & $S$. viridochromogenes & ${ }^{*}$ DSM 41519 & & & Category III Species no. 04 \\
\hline 9 & $1-19$ & S. viridochromogenes & ${ }^{*}$ DSM 41522 & ATCC 21240 & & Category III Species no. 04 \\
\hline 9 & $1-19$ & S. viridochromogenes & * DSM 41523 & ATCC 21724 & & Category III Species no. 04 \\
\hline 9 & $1-19$ & S. viridochromogenes & *DSM 41587 & NRRL B-3027 & & Category III Species no. 04 \\
\hline 9 & $1-19$ & S. viridochromogenes & *DSM 41590 & NRRL B-3607 & & Category III Species no. 04 \\
\hline 9 & $1-19$ & S. viridochromogenes & ${ }^{*}$ DSM 41591 & NRRL B-12033 & & Category III Species no. 04 \\
\hline 9 & $1-19$ & S. viridochromogenes & *DSM 41589 & NRRL B-3263 & & Category III Species no. 04 \\
\hline 9 & $1-19$ & $S$. yokosukanensis & ${ }^{*}$ DSM $40224^{(T)}$ & ATCC 25520 ISP 5224 & A 30 & Category II Species no. 06 \\
\hline \multicolumn{7}{|c|}{ Strains assigned to cluster 10: Streptomyces sparsogenes (2) } \\
\hline 10 & $1-29$ & S. hygroscopicus & *DSM 41532 & ATCC 31050 & & Category I Species no. 16 \\
\hline 10 & $1-29$ & S. sparsogenes & ${ }^{*}$ DSM $40356^{(\mathrm{T})}$ & ATCC 25498 ISP 5356 & A 32 & Category I Species no. 16 \\
\hline \multicolumn{7}{|c|}{ Strains assigned to cluster 11: Streptomyces psammoticus (5) } \\
\hline 11 & $1-20$ & S. aureomonopodiales & DSM 40914 & & & \\
\hline 11 & $1-20$ & S. parvulus & *DSM 40912 & & & \\
\hline 11 & $1-21$ & S. psammoticus & ${ }^{*} \mathrm{DSM} 40341^{\mathrm{T}}$ & ATCC 25488 ISP 5341 & F 67 & Category II Species no. 17 \\
\hline 11 & $1-20$ & S. rochei & *DSM 40913 & & & Category I Species no. 07 \\
\hline 11 & $1-20$ & S. viridochromogenes & ${ }^{*}$ DSM 41515 & & & Category III Species no. 04 \\
\hline \multicolumn{7}{|c|}{ Strains assigned to cluster $12:$ Streptomyces aurantiacus (2) } \\
\hline 12 & 19 & S. aurantiacus & ${ }^{*}$ DSM $40412^{\mathrm{T}}$ & ATCC 19822 ISP 5412 & C 45 & Category II Species no. 13 \\
\hline 12 & 19 & S. tauricus & DSM 40560 & ATCC 27470 ISP 5560 & A 19 & \\
\hline \multicolumn{7}{|c|}{ Strains assigned to cluster 13: Streptomyces niveoruber (9) } \\
\hline 13 & $1-19$ & S. albospinus & *DSM 41422 & & & Category IV Species no. 03 (grey series) \\
\hline 13 & $1-19$ & $\begin{array}{l}\text { S. cyanoglomerus subsp. } \\
\text { cellulosae }\end{array}$ & *DSM 40427 & ATCC 19837 ISP 5427 & & \\
\hline 13 & $1-19$ & S. ederensis & ${ }^{*}$ DSM $40741^{\mathrm{T}}$ & ATCC 15304 & & Category IV Species no. 14 (grey series) \\
\hline 13 & $1-19$ & S. griseoruber & *DSM 40769 & & & Category I Species no. 14 \\
\hline 13 & $1-18$ & S. hygroscopicus & ${ }^{*}$ DSM 40632 & NRRL B-1503 & & \\
\hline 13 & $1-19$ & $S$. niveoruber & ${ }^{*} \mathrm{DSM} 40638^{\mathrm{T}}$ & ATCC 14971 & & Category IV Species no. 08 (red series) \\
\hline 13 & $1-19$ & S. scabies & DSM 40994 & & & \\
\hline 13 & $1-19$ & Streptomyces $\mathrm{sp}$. & DSM 40989 & & & \\
\hline 13 & $1-19$ & S. toyocaensis & DSM 40030 & ATCC 19814 ISP 5030 & A 29 & \\
\hline \multicolumn{7}{|c|}{ Strains assigned to cluster $14:$ Streptomyces sp. (2) } \\
\hline 14 & 21 & S. albus & *DSM 40832 & NRRL 2490 & & Species no. 09 \\
\hline 14 & 21 & S. scabies & DSM 40962 & & & \\
\hline \multicolumn{7}{|c|}{ Strain assigned to cluster 15 : Streptomyces massasporeus (1) } \\
\hline 15 & $1-19$ & S. massasporeus & ${ }^{*} \mathrm{DSM} 40035^{\mathrm{T}}$ & ATCC 19785 ISP 5035 & D SM & Category III Species no. 19 \\
\hline \multicolumn{7}{|c|}{ Strain assigned to cluster 16: Streptomyces lanatus (1) } \\
\hline 16 & $1-19$ & S. lanatus & ${ }^{*}$ DSM $40090^{(\mathrm{T})}$ & ATCC 19775 ISP 5090 & A 18 & Species no. 11 \\
\hline \multicolumn{7}{|c|}{ Strains assigned to cluster $17:$ Streptomyces griseoloalbus (2) } \\
\hline 17 & 7 & S. flaviscleroticus & ${ }^{*}$ DSM $40270^{(\mathrm{T})}$ & & & Category I Species no. 08 \\
\hline 17 & 7 & S. griseoloalbus & $*$ DSM $40468^{T}$ & ATCC 23624 ISP 5468 & & Category IV Species no. 05 (yellow serie \\
\hline \multicolumn{7}{|c|}{ Strain assigned to cluster $18:$ Streptomyces griseoruber (1) } \\
\hline 18 & 23 & S. griseoruber\# & $*$ DSM $40281^{T}$ & ATCC 23919 ISP 5281 & A 21 & Species no. 14 \\
\hline
\end{tabular}


Table 1 (continued)

\begin{tabular}{|c|c|c|c|c|c|}
\hline$s_{\mathrm{SM}}{ }^{+}$ & $S_{\mathrm{J}} \ddagger$ & Strain designation $\S$ & Strain numbers $\|$ & $\begin{array}{l}\text { Williams } \\
\text { group }\end{array}$ & $\begin{array}{l}\text { Bergey category } \\
\text { and species no. }+\dagger\end{array}$ \\
\hline
\end{tabular}

1923 S. cyaneogriseus

2032 S. albidoflavus

$20 \quad$ 1-20 S. canadiensis

2032 S. celluloflavus

2032 S. chromogenes

2032 S. chrysomallus

$20 \quad 1-8$ S. griseobrunneus

$21 \quad 1-5 \quad$ S. anandii

$21 \quad 1-5$ Streptomyces sp.

$\begin{array}{lll}21 & 2 & S . \text { thermonitrificans }\end{array}$

$21 \quad 2$ S. thermovulgaris

212 S. thermovulgaris

Strain assigned to cluster 19: Streptomyces cyaneogriseus (1)

$$
\text { DSM } 40534 \quad \text { ISP } 5534 \text { H Sm }
$$

Strains assigned to cluster 20: Streptomyces chrysomallus (6)

*DSM 40880

DSM 40837 ATCC 17776

*DSM 40839' ATCC 29806

Category I Species no. 01

DSM 40765

*DSM 40870

*DSM 40915

Category IV Species no. 01 (yellow series)

Category I Species no. 02

Strains assigned to cluster 21: Streptomyces thermovulgaris (5)

${ }^{*}$ DSM 40535(T) ATCC 19388 ISP 5535 B 42 Category I Species no. 19

DSM 40980

${ }^{*}$ DSM 40579(T) ATCC 23385 ISP 5579 A 36 Category II Species no. 09

${ }^{*}$ DSM 40444(T) ATCC 19284 ISP 5444 A 36 Category II Species no. 09

*DSM 40787 Category II Species no. 09

Strains assigned to cluster 22: Streptoverticillium sp./Streptomyces lavendulae/Streptomyces fradiae

Strains assigned to subcluster 22-1: Streptoverticillium griseocarneum (51)

Strains

$22-1$

$22-1$

$22-1$

$22-1$

$22-1$

$22-1$

$22-1$

$22-1$

$22-1$

$22-1$

22-1

22-1

22-1

22-1

22-1

22-1

22-1

22-1

22-1

22-1

22-1

22-1

22-1

22-1

22-1

22-1

22-1

22-1

22-1

22-1

22-1

22-1

22-1

22-1

22-1

22-1

22-1

22-1

22-1

22-1

22-1

22-1
$40 \mathrm{~Sv}$. flavopersicum

40 Sv. griseoverticillatum

40 Sv. mediocidicus

40 Sv ardum

$40 S v$. aspergilloides

40 Sv. aureoversales

40 Sv. baldaccii

40 Sv. biverticillatum

40 Sv. blastmyceticum

40 Sv. caespitosum

40 Sv. cinnamoneum subsp. albosporum

40 Sv. cinnamoneum

$40 S v$. cinnamoneum subsp. azocolutum

40 Sv. cinnamoneum subsp. lanosum

40 Sv. cinnamoneum subsp. sparsum

40 Sv. distallicum

40 Sv. ehimense

40 Sv. eurocidicum

40 Sv. fervens subsp. fervens

40 Sv. fervens subsp. melrosporus

40 Sv. griseocarneum

40 Sv. hachijoense

40 Sv. hiroshimense

40 Sv. kentuckense

40 Sv. kishiwadense

40 Sv. lilacinum

40 Sv. luteoverticillatum

40 Sv. mashuense

40 Sv. mashuense

40 Sv. mobaraense

$40 \mathrm{~Sv}$. morookaensis

40 Sv. netropsis

$40 \mathrm{~Sv}$. orinoci

40 Sv. parvisporogenum

40 Sv. paucisporogenum

40 Sv. pentaticum subsp. jenense

40 Sv. rectiverticillatum

$40 S v$, reticulum

$40 S v$. reticulum subsp. protomycicum

40 Sv. roseoverticillatum

$40 S v$, roseoverticillatum subsp. albosporum

40 Sv. rubroverticillatum

40 Sv. salmonis
*DSM 40093(T) ATCC 19756 ISP 5093

*DSM $40507^{(T)}$

DSM 40021 ATCC 23936 ISP 5021

*DSM 40527(T) ATCC 27417 ISP 5527

DSM 40565 ATCC 14808 ISP 5565

${ }^{*}$ DSM $40387^{(\mathrm{T})} \quad$ ATCC 15853 ISP 5387

*DSM 40845 $\quad$ ATCC 23654

*DSM 40272(T) ATCC 23615 ISP 5272

*DSM 40029(T) ATCC 19731 ISP 5029

DSM 40603 ATCC 27442 ISP 5603

*DSM 40897(T) ATCC 25186

${ }^{*}$ DSM 40005 $\quad$ ATCC 11874 ISP 5005

DSM 40646 ATCC 12686

*DSM 40898(T) ATCC 25187

*DSM 40899(T) ATCC 25185

*DSM 40846(T) NCIB 8936

*DSM 40253(T) ATCC 23903 ISP 5253

${ }^{*}$ DSM 40604(T) ATCC 27428 ISP 5604

*DSM 40086(T) ATCC 27429 ISP 5086

*DSM 40905(T) NRRL 3117

*DSM 40004 ${ }^{\mathrm{T}}$ ATCC 12628 ISP 5004

* DSM 40114 ${ }^{\mathrm{T}}$ ATCC 19769 ISP 5114

* DSM 40037(T) ATCC 19772 ISP 5037

*DSM 40052(T) ATCC 12691 ISP 5052

*DSM 40397 $\quad$ ATCC 25464 ISP 5397

*DSM 40254 ${ }^{\mathrm{T}} \quad$ ATCC 23930 ISP 5254

*DSM 40038(T) ATCC 23933 ISP 5038

*DSM 40221(T) ATCC 23934 ISP 5221

*DSM 40896(T)

*DSM 40847

DSM $40503^{\mathrm{T}}$

*DSM 40259(T)

*DSM 40571 ${ }^{\mathrm{T}}$

*DSM 40473(T)

DSM 40315

DSM 40848

*DSM $40436^{\mathrm{T}}$

DSM 40893

ATCC 29032

ATCC 19166 ISP 5503

ATCC 23940 ISP 5259

ATCC 23202 ISP 5571

ATCC 12568 ISP 5473

ATCC 12596 ISP 5315

ATCC 19845 ISP 5436

ATCC 25607

DSM 40849

${ }^{*}$ DSM 40039(T) ATCC 19807 ISP 5039

DSM 40900

DSM 40851

*DSM 40895(T) NRRL B-1472
F 56 Streptov.

F 58 Streptov.

F Sm Streptov.

Streptov.

Streptov.

Streptov.

Streptov.

Streptov.

F 58 Streptov.

Streptov.

Streptov.

F 55 Streptov.

Streptov.

Streptov.

Streptov.

Streptov.

Streptov.

F 56 Streptov.

Streptov.

Streptov.

F 55 Streptov.

F 55 Streptov.

F 57 Streptov.

F SM Streptov.

Streptov.

Streptov.

F 55 Streptov.

F 55 Streptov.

Streptov.

Streptov.

F 59 Streptov.

F 56 Streptov.

F 58 Streptov.

Streptov.

F 55 Streptov.

Streptov.

F 57 Streptov.

Streptov.

Streptov.

Streptov.

Species no. 01 Species no. 01 Species no. 02 Species no. 03 Species no. 08 Species no. 05 Species no. 01 Species no. 01 Species no. 02

Species no. 02 Species no. 02

Species no. 03 Species no. 02 Species no. 02 Species no. 01 Species no. 09 Species no. 02 Species no. 01 Species no. 01 Species no. 03 Species no. 04 Species no. 01 Species no. 11 Species no. 15 Species no. 16 Species no. 03 Species no. 03 Species no. 03 Species no. 07 Species no. 08 Species no. 01 Species no. 17 Species no. 02 Species no. 02 Species no. 03 Species no. 18 Species no. 19

Streptov. Streptov. Streptov.
Species no. 19

Species no. 01

Species no. 01 Species no. 01 Species no. 05 
Table 1 (continued)

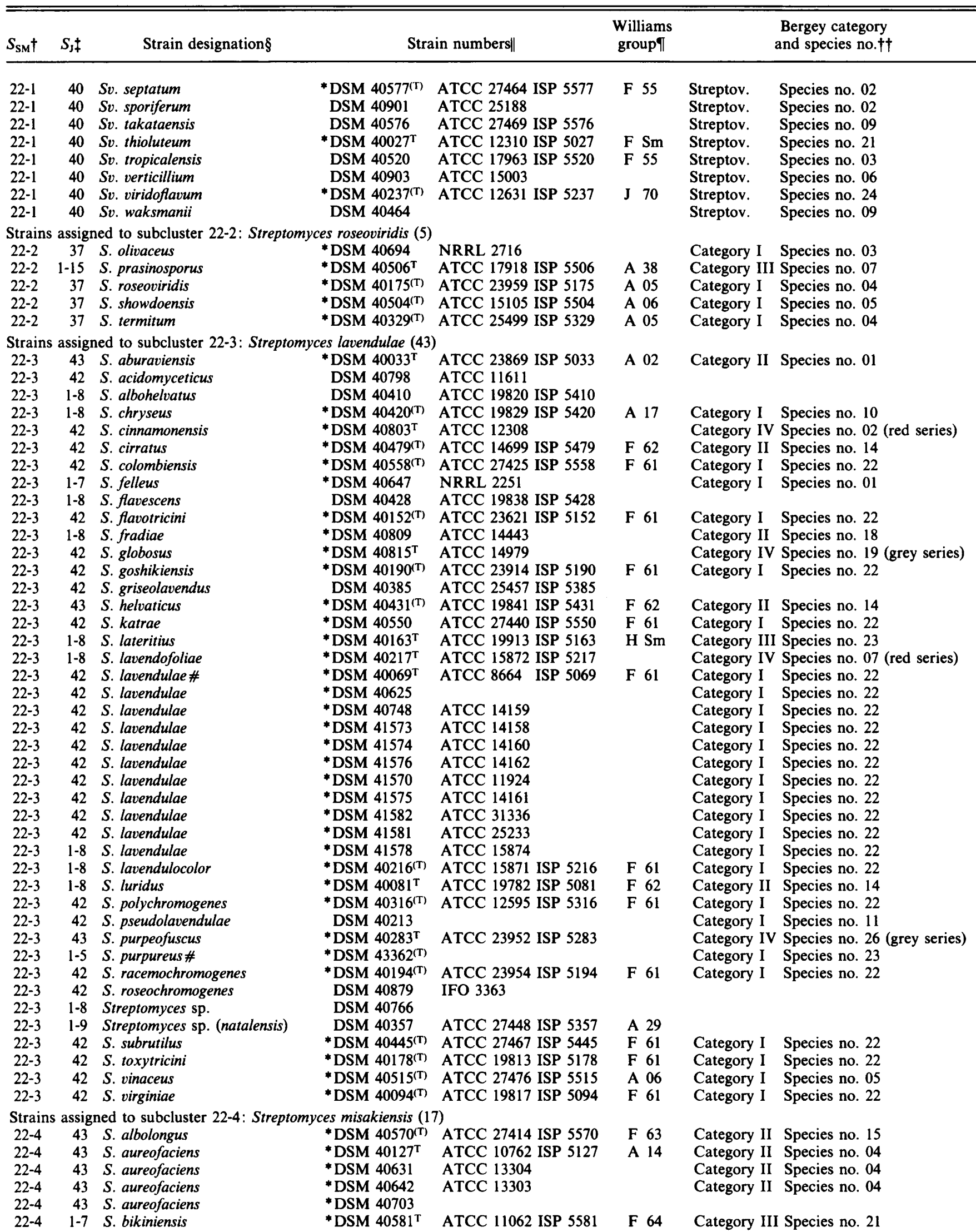


Table 1 (continued)

\begin{tabular}{|c|c|c|c|c|c|c|c|}
\hline$S_{\mathrm{SM}} \dagger$ & $S_{\mathrm{J}} \ddagger$ & Strain designation $\S$ & Stra & ain numbers & $\begin{array}{l}\text { Williams } \\
\text { groupI }\end{array}$ & & $\begin{array}{l}\text { Bergey category } \\
\text { and species no. }+\dagger\end{array}$ \\
\hline $22-4$ & $1-6$ & S. cuspidosporus & ${ }^{*}$ DSM 41424 & & & Category IV & Species no. 11 (grey series) \\
\hline $22-4$ & 43 & S. finlayi & ${ }^{*}$ DSM $40218^{T}$ & ATCC 23340 ISP 5218 & I $\mathrm{Sm}$ & Category III & Species no. 24 \\
\hline $22-4$ & 43 & S. fradiae & ${ }^{*}$ DSM 40810 & ATCC 15438 & & Category II & Species no. 18 \\
\hline $22-4$ & 43 & S. herbaricolor & ${ }^{*}$ DSM $40123^{(\mathrm{T})}$ & ATCC 23922 ISP 5123 & A 02 & Category II & Species no. 01 \\
\hline $22-4$ & 35 & S. karnatakensis & DSM 40345 & ATCC 25463 ISP 5345 & C 44 & & \\
\hline $22-4$ & 43 & S. misakiensis & ${ }^{*}$ DSM $40222^{T}$ & ATCC 23938 ISP 5222 & F 66 & Category II & Species no. 16 \\
\hline $22-4$ & 35 & S. pactum & ${ }^{*}$ DSM $40530^{\mathrm{T}}$ & ATCC 27456 ISP 5530 & C 44 & Category II & Species no. 12 \\
\hline $22-4$ & 62 & 'S. pyridomyceticus' & DSM 40024 & ATCC 23953 ISP 5024 & & & \\
\hline $22-4$ & $1-7$ & S. venezuelae & ${ }^{*}$ DSM 40755 & & & Category I & Species no. 05 \\
\hline $22-4$ & 43 & $S$. viridifaciens & DSM 40239 & ATCC 11989 ISP 5239 & F 66 & & \\
\hline $22-4$ & 43 & S. xanthocidicus & ${ }^{*}$ DSM $40575^{(\mathrm{T})}$ & ATCC 27480 ISP 5575 & F 66 & Category II & Species no. 16 \\
\hline \multicolumn{8}{|c|}{ Strains assigned to subcluster 22-5: Streptomyces fradiae (17) } \\
\hline $22-5$ & 39 & S. albus & ${ }^{*}$ DSM 40950 & & & Category I & Species no. 09 \\
\hline $22-5$ & 39 & S. clavuligerus & ${ }^{*}$ DSM $40751^{\mathrm{T}}$ & ATCC 27064 & & Category IV & Species no. 10 (grey series) \\
\hline $22-5$ & 39 & S. fradiae & ${ }^{*}$ DSM $40063^{T}$ & ATCC 10745 ISP 5063 & G 68 & Category II & Species no. 18 \\
\hline $22-5$ & 39 & S. fradiae & *DSM 40877 & & & Category II & Species no. 18 \\
\hline $22-5$ & 39 & $S$. fradiae & ${ }^{*}$ DSM 40953 & & & Category II & Species no. 18 \\
\hline $22-5$ & 39 & S. fradiae & *DSM 40955 & & & Category II & Species no. 18 \\
\hline $22-5$ & 39 & S. fradiae & ${ }^{*}$ DSM 40956 & & & Category II & Species no. 18 \\
\hline $22-5$ & 39 & S. fradiae & ${ }^{*}$ DSM 40952 & & & Category II & Species no. 18 \\
\hline $22-5$ & 39 & S. fradiae & ${ }^{*}$ DSM 40954 & & & Category II & Species no. 18 \\
\hline $22-5$ & 39 & S. fradiae & *DSM 40957 & & & Category II & Species no. 18 \\
\hline $22-5$ & 39 & S. fradiae & *DSM 40958 & & & Category II & Species no. 18 \\
\hline $22-5$ & 39 & S. fradiae & ${ }^{*}$ DSM 41543 & ATCC 11903 & & Category II & Species no. 18 \\
\hline $22-5$ & 39 & S. fradiae & ${ }^{*}$ DSM 41551 & ATCC 21896 & & Category II & Species no. 18 \\
\hline $22-5$ & 39 & S. fradiae & ${ }^{*}$ DSM 41545 & ATCC 19063 & & Category II & Species no. 18 \\
\hline $22-5$ & 39 & S. fradiae & ${ }^{*} \mathrm{DSM} 41550$ & ATCC 21401 & & Category II & Species no. 18 \\
\hline $22-5$ & $1-5$ & S. roseoflavus & *DSM 40536 & ATCC 13167 ISP 5536 & & Category IV & Species no. 10 (red series) \\
\hline $22-5$ & 39 & S. roseolilacinus & ${ }^{*}$ DSM $40173^{(T)}$ & ATCC 19922 ISP 5173 & G 68 & Category II & Species no. 18 \\
\hline \multicolumn{8}{|c|}{ Strains assigned to cluster 23: Streptomyces griseolus (2) } \\
\hline 23 & 4 & Sv. avidinii & ${ }^{*}$ DSM $40526^{(\mathrm{T})}$ & ATCC 27419 ISP 5526 & F 56 & Streptov. & Species incertae sedis \\
\hline 23 & 4 & S. griseolus & *DSM 40854 & ATCC 11796 & & Category I & Species no. 03 \\
\hline \multicolumn{8}{|c|}{ Strains assigned to cluster 24: Streptomyces fungicidicus (4) } \\
\hline 24 & 5 & S. fungicidicus & DSM 40811 & ATCC 13853 & & & \\
\hline 24 & 56 & S. hygroscopicus & *DSM 41527 & ATCC 21705 & & Category I & Species no. 16 \\
\hline 24 & 56 & S. hygroscopicus & ${ }^{*}$ DSM 41604 & NRRL B-16256 & & Category I & Species no. 16 \\
\hline 24 & 57 & Streptomyces sp. & DSM 40987 & & & & \\
\hline \multicolumn{8}{|c|}{ Strains assigned to cluster 25: Streptomyces lydicus (17) } \\
\hline 25 & $1-9$ & S. albulus & ${ }^{*}$ DSM $40492^{(\mathrm{T})}$ & ATCC 12757 ISP 5492 & A 29 & Category I & Species no. 15 \\
\hline 25 & 5 & S. bottropensis & *DSM 40800 & ATCC 13854 & & Category I & Species no. 12 \\
\hline 25 & 5 & S. fasiculatus & DSM 40054 & ATCC 19751 ISP 5054 & A 29 & & \\
\hline 25 & 5 & S. hygroscopicus & ${ }^{*}$ DSM 40823 & & & Category I & Species no. 16 \\
\hline 25 & 5 & S. hygroscopicus & ${ }^{*}$ DSM 41525 & ATCC 15869 & & Category I & Species no. 16 \\
\hline 25 & 5 & S. hygroscopicus & *DSM 41526 & ATCC 21582 & & Category I & Species no. 16 \\
\hline 25 & 5 & S. hygroscopicus & ${ }^{*}$ DSM 41528 & ATCC 21722 & & Category I & Species no. 16 \\
\hline 25 & $1-15$ & S. kurssanovii & DSM $40162^{\mathrm{T}}$ & ATCC 15824 ISP 5162 & F 60 & Category IV & Species no. 20 (grey series) \\
\hline 25 & 5 & S. libani & ${ }^{*} \mathrm{DSM} 40555^{(\mathrm{T})}$ & ATCC 23732 ISP 5555 & A 29 & Category I & Species no. 15 \\
\hline 25 & 5 & S. lydicus\# & ${ }^{*}$ DSM $40461^{T}$ & ATCC 25470 ISP 5461 & A 29 & Category I & Species no. 15 \\
\hline 25 & 5 & S. nigrescens & ${ }^{*}$ DSM $40276^{(\mathrm{T})}$ & ATCC 23941 ISP 5276 & A 29 & Category I & Species no. 15 \\
\hline 25 & $1-9$ & $S$. noursei & ${ }^{*} \mathrm{DSM} 40635^{\mathrm{T}}$ & ATCC 11455 & & Category IV & Species no. 23 (grey series) \\
\hline 25 & $1-23$ & S. ostreogriseus & DSM 40511 & ATCC 27455 ISP 5511 & A 25 & & \\
\hline 25 & 5 & S. platensis & ${ }^{*} \mathrm{DSM} 40041^{(\mathrm{T})}$ & ATCC 13865 ISP 5041 & A 29 & Category I & Species no. 15 \\
\hline 25 & 5 & S. sioyaensis & ${ }^{*} \mathrm{DSM} 40032^{(\mathrm{T})}$ & ATCC 13989 ISP 5032 & A 29 & Category I & Species no. 15 \\
\hline 25 & $1-9$ & Streptomyces sp. (chromogenus) & DSM 40384 & ATCC 27424 ISP 5284 & A 33 & & \\
\hline 25 & 5 & S. tubercidicus & ${ }^{*}$ DSM $40261^{\mathrm{T}}$ & ATCC 25502 ISP 5261 & C 47 & Category III & Species no. 14 \\
\hline \multicolumn{8}{|c|}{ Strains assigned to cluster 26: Streptomyces flavidovirens (2) } \\
\hline 26 & 33 & S. flavidovirens & ${ }^{*}$ DSM $40150^{\mathrm{T}}$ & ATCC 19900 ISP 5150 & & Category IV & Species no. 03 (yellow series) \\
\hline 26 & 27 & S. tenebrarius & DSM 40477 & ATCC 17920 ISP 5477 & & & \\
\hline \multicolumn{8}{|c|}{ Strains assigned to cluster $27:$ Streptomyces fradiae (2) } \\
\hline $\begin{array}{l}27 \\
27\end{array}$ & 31 & S. fradiae & *DSM 41549 & ATCC 21098 & & Category II & Species no. 18 \\
\hline 27 & 31 & S. fradiae & *DSM 41548 & ATCC 21097 & & Category II & Species no. 18 \\
\hline
\end{tabular}


Table 1 (continued)

\begin{tabular}{|c|c|c|c|c|c|}
\hline$S_{\mathrm{SM}}^{\dagger}$ & $S_{\mathrm{J}} \ddagger$ & Strain designation $\S$ & Strain numbers" & $\begin{array}{l}\text { Williams } \\
\text { group }\end{array}$ & $\begin{array}{l}\text { Bergey category } \\
\text { and species no. } \dagger \dagger\end{array}$ \\
\hline
\end{tabular}

28

9 S. achromogenes subsp. rubradiris

28

9 S. prasinus

$29 \quad$ 1-32 S. loidensis

$\begin{array}{lll}30 & 1-34 & \text { S. albus } \\ 30 & 1-34 & \text { S. albus } \\ 30 & 1-34 & \text { S. albus } \\ 30 & 1-34 & \text { S. albus } \\ 30 & 1-34 & \text { S. albus } \\ 30 & 1-34 & \text { S. albus } \\ 30 & 1-34 & \text { S. albus } \\ 30 & 1-34 & \text { S. albus } \\ 30 & 1-34 & \text { S. albus } \\ 30 & 1-34 & \text { S. albus } \\ 30 & 1-34 & \text { S. almquistii } \\ 30 & 1-34 & \text { S. antibioticus } \\ 30 & 1-34 & \text { S. flocculus } \\ 30 & 1-34 & \text { S. flocculus } \\ 30 & 1-34 & \text { S. gibsonii } \\ 30 & 1-34 & \text { S. rangoon } \\ 30 & 1-34 & \text { S. rimosus }\end{array}$

$\begin{array}{rrl}31 & 1-34 & S . \text { aminophilus } \\ 31 & 46 & \text { S. antibioticus }\end{array}$

31 1-34 S. cacaoi

$32 \quad 27$ S. albus

$$
\begin{array}{ll}
1-33 & \text { S. alboflavus } \\
1-33 & \text { S. aureocirculatus } \\
1-33 & \text { S. aureofasciculus } \\
1-33 & \text { S. aureomonopodiales } \\
1-33 & \text { S. aureoverticillatus } \\
1-33 & \text { S. cinnamonensis } \\
1-33 & S . \text { flavofungini } \\
1-33 & \text { S. kanamyceticus } \\
1-33 & \text { S. kanamyceticus } \\
1-33 & \text { S. kanamyceticus } \\
1-33 & \text { S. kanamyceticus } \\
1-33 & \text { S. kanamyceticus } \\
1-33 & S . \text { longispororuber } \\
1-33 & \text { S. longispororuber } \\
1-33 & \text { S. longissimus } \\
1-33 & \text { S. moderatus } \\
1-33 & \text { S. nobilis } \\
1-33 & \text { S. paraguayensis } \\
1-33 & \text { S. venezuelae }
\end{array}
$$

$$
\begin{aligned}
1-33 & \text { S. albofaciens } \\
1-33 & \text { S. alboflavus } \\
1-33 & \text { S. capuensis } \\
41 & \text { S. catenulae } \\
1-33 & \text { S. chrestomyceticus }
\end{aligned}
$$

Strains assigned to cluster 28: Streptomyces prasinus (2)

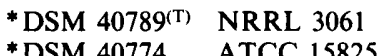

Strain assigned to cluster 29: Streptomyces loidensis (1)

$$
\text { DSM } 40825 \text { ATCC } 11415
$$

Strains assigned to cluster 30: Streptomyces albus (17)

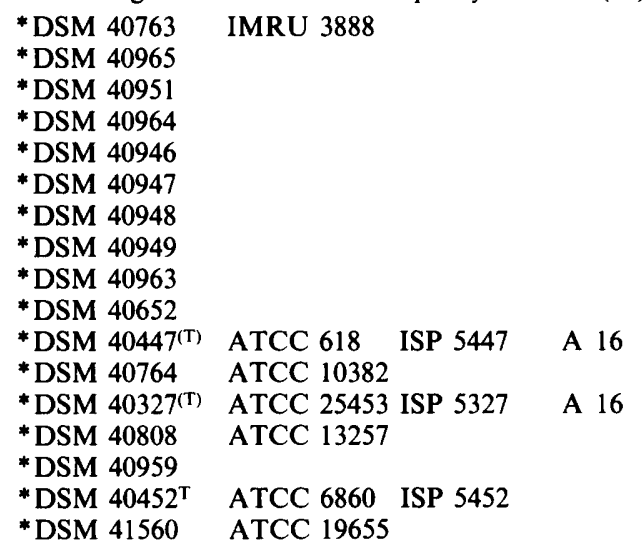

Strains assigned to cluster 31 : Streptomyces cacaoi (3)

*DSM 40186(T) ATCC 14961 ISP 5186 A 16

*DSM 41568 ATCC 15849

${ }^{*}$ DSM $40057^{(T)}$ ATCC 3082 ISP 5057 A 16

Strain assigned to cluster 32: Streptomyces albus (1)

${ }^{*}$ DSM $40313^{\mathrm{T}} \quad$ ATCC 3004 ISP 5313 A 16
Category I Species no. 12

Category I Species no. 17
Category I Species no. 09

Category I Species no. 09

Category I Species no. 09

Category I Species no. 09

Category I Species no. 09

Category I Species no. 09

Category I Species no. 09

Category I Species no. 09

Category I Species no. 09

Category I Species no. 09

Category I Species no. 09

Category I Species no. 21

Category I Species no. 09

Category I Species no. 09

Category IV Species no. 05 (white series)

Category IV Species no. 07 (white series)

Category I Species no. 19

Category I Species no. 09

Category I Species no. 21

Category I Species no. 09

Category I Species no. 09
Strains assigned to cluster 33: Streptomyces alboflavus (19)
${ }^{*}$ DSM 40045 ${ }^{\mathrm{T}}$ ATCC 12626 ISP 5045

*DSM 40386(T) ATCC 19823 ISP 5386

DSM 40414 ATCC 19824 ISP 5414

DSM 40416 ATCC 19825 ISP 5416

*DSM 40080(T) ATCC 15854 ISP 5080

*DSM 40804 ATCC 15413

DSM 40366 ATCC 27430 ISP 5366

*DSM 41555 ATCC 21268

*DSM 41552 ATCC 21259

*DSM 41554 ATCC 21261

*DSM $41556 \quad$ ATCC 21486

*DSM $41553 \quad$ ATCC 21260

*DSM 40749 ATCC 13931

*DSM 40599(T) ATCC 27443 ISP 5599

DSM 40435 ATCC 19850 ISP 5435

DSM 40529 ATCC 23443 ISP 5529

DSM 40441 ATCC 19251 ISP 5441

DSM 40567 ATCC 27458 ISP 5567
E 54

Category III Species no. 20

E 54

A 10 Category I Species no. 06

B 42

Category IV Species no. 02 (red series)

Category I Species no. 19

Category I Species no. 19

Category I Species no. 19

Category I Species no. 19

Category I Species no. 19

Category I Species no. 06

A 10 Category I Species no. 06

E 54

A Sm

A 10

C 46
Category I Species no. 05

Strain assigned to cluster 34: Streptomyces fulvissimus (1)

${ }^{*}$ DSM $40593^{\mathrm{T}} \quad$ ATCC 27431 ISP 5593 A 10 Category I Species no. 06

Strains assigned to cluster 35: Streptomyces rimosus (24)

${ }^{*}$ DSM 40268(T) ATCC 25184 ISP 5268 B 42 Category I Species no. 19

*DSM 40761 NCIB 9453

DSM 40402 ATCC 25436 ISP 5402 B 42

*DSM 40258(T) ATCC 12476 ISP 5258 C 43 Category II Species no. 11

${ }^{*}$ DSM 40545(T) ATCC 14947 ISP 5545 B 42 Category I Species no. 19 
Table 1 (continued)

\begin{tabular}{|c|c|c|c|c|c|c|c|}
\hline$S_{\text {SM }}{ }^{\dagger}$ & $S_{\mathrm{J}} \ddagger$ & Strain designation $\S$ & \multicolumn{2}{|c|}{ Strain numbers $\|$} & \multirow[t]{2}{*}{$\begin{array}{l}\text { Williams } \\
\text { group } \Phi\end{array}$} & \multicolumn{2}{|r|}{$\begin{array}{l}\text { Bergey category } \\
\text { and species no. } \dagger \dagger\end{array}$} \\
\hline 35 & $1-33$ & S. djakartensis & ${ }^{*}$ DSM $40743^{T}$ & ATCC 13441 & & Category IV & Species no. 12 (grey series) \\
\hline 35 & $1-33$ & S. erumpens & - DSM $40941^{\mathrm{T}}$ & ATCC 23266 & & Category IV & Species no. 15 (grey series) \\
\hline 35 & $1-33$ & S. fungicidicus & DSM 40020 & ATCC 27432 ISP 5020 & A 16 & & \\
\hline 35 & $1-33$ & S. humifer & DSM 40602 & ATCC 13748 ISP 5602 & A $1 C$ & & \\
\hline 35 & $1-33$ & S. hygroscopicus & ${ }^{*}$ DSM 40821 & ATCC 13810 & & & \\
\hline 35 & $1-33$ & S. krestomyceticus & DSM 40820 & & & & \\
\hline 35 & $1-33$ & S. mauvecolor & *DSM 40827 & & & Category IV & Species no. 08 (violet series) \\
\hline 35 & $1-33$ & $S$. peucetius & *DSM 40754 & NCIB 10972 & & Category IV & Species no. 09 (red series) \\
\hline 35 & 41 & S. ramulosus & ${ }^{*} \mathrm{DSM} 40100^{\mathrm{T}}$ & ATCC 19802 ISP 5100 & C Sm & Category III & I Species no. 16 \\
\hline 35 & $1-33$ & S. rimosus\# & ${ }^{*} \mathrm{DSM} 40260^{\mathrm{T}}$ & ATCC 10970 ISP 5260 & B 42 & Category I & Species no. 19 \\
\hline 35 & $1-33$ & S. rimosus & *DSM 40673 & & & Category I & Species no. 19 \\
\hline 35 & $1-33$ & S. rimosus & *DSM 41557 & ATCC 13224 & & Category I & Species no. 19 \\
\hline 35 & 1.33 & S. rimosus & *DSM 41559 & ATCC 14673 & & Category I & Species no. 19 \\
\hline 35 & $1-33$ & S. rimosus & *DSM 41562 & ATCC 23307 & & Category I & Species no. 19 \\
\hline 35 & $1-33$ & S. rimosus & ${ }^{*}$ DSM 41563 & ATCC 33022 & & Category I & Species no. 19 \\
\hline 35 & $1-33$ & S. rimosus & *DSM 41558 & ATCC 14500 & & Category I & Species no. 19 \\
\hline 35 & $1-33$ & S. rimosus & *DSM 41561 & ATCC 19656 & & Category I & Species no. 19 \\
\hline 35 & $1-33$ & S. somaliensis & ${ }^{*}$ DSM 40760 & ATCC 14817 & & & \\
\hline 35 & $1-33$ & S. vendargensis & DSM 40379 & ATCC 25507 ISP 5379 & & & \\
\hline \multicolumn{6}{|c|}{ Strains assigned to cluster 36 : Streptomyces rimosus (2) } & & \\
\hline 36 & 45 & S. rimosus & *DSM 41565 & ATCC 33024 & & Category I & Species no. 19 \\
\hline 36 & 45 & S. rimosus & * DSM 41564 & ATCC 33023 & & Category I & Species no. 19 \\
\hline \multicolumn{8}{|c|}{ Strain assigned to cluster 37 : Streptomyces varsoviensis (1) } \\
\hline 37 & 028 & S. varsoviensis & ${ }^{*}$ DSM $40346^{\mathrm{T}}$ & ATCC 25505 ISP 5346 & C 46 & Category III & I Species no. 13 \\
\hline \multicolumn{8}{|c|}{ Strain assigned to cluster 38: Actinomadura luteofluorescens (1) } \\
\hline 38 & 025 & A. luteofluorescens & ${ }^{*}$ DSM $40398^{(T)}$ & ATCC 25469 ISP 5398 & & & \\
\hline \multicolumn{8}{|c|}{ Strain assigned to cluster 39: Streptomyces caelicus (1) } \\
\hline 39 & $1-28$ & S. caelicus & DSM 40835 & NRRL 2957 & & & \\
\hline \multicolumn{8}{|c|}{ Strain assigned to cluster 40: Streptomyces spheroides (1) } \\
\hline 40 & 48 & S. spheroides & ${ }^{*}$ DSM $40292^{(T)}$ & ATCC 23965 ISP 5292 & A 1 B & Category I & Species no. 02 \\
\hline \multicolumn{8}{|c|}{ Strains assigned to cluster 41 : Streptomyces endus (2) } \\
\hline 41 & 12 & S. endus & ${ }^{*}$ DSM $40187^{(\mathrm{T})}$ & ATCC 23904 ISP 5187 & A 32 & Category I & Species no. 16 \\
\hline 41 & 12 & S. violaceusniger & *DSM 40699 & & & Category I & Species no. 16 \\
\hline \multicolumn{8}{|c|}{ Strain assigned to cluster $42:$ Streptomyces olivaceus (1) } \\
\hline 42 & 14 & S. olivaceus & ${ }^{*}$ DSM $40072^{(T)}$ & ATCC 3335 ISP 5072 & A $1 C$ & Category I & Species no. 03 \\
\hline \multicolumn{8}{|c|}{ Strain assigned to cluster 43: Streptomyces niveus (1) } \\
\hline 43 & 13 & $S$. niveus & ${ }^{*}$ DSM $40088^{(T)}$ & ATCC 19793 ISP 5088 & A 1 B & Category I & Species no. 02 \\
\hline \multicolumn{8}{|c|}{ Strains assigned to cluster $44:$ Streptomyces sp. (2) } \\
\hline 44 & $1-24$ & Streptomyces sp. & DSM 40974 & & & & \\
\hline 44 & $1-24$ & Streptomyces sp. & DSM 40982 & & & & \\
\hline \multicolumn{8}{|c|}{ Strain assigned to cluster 45 : Streptomyces scabies (1) } \\
\hline 45 & $1-24$ & S. scabies & *DSM 40961 & & & & \\
\hline \multicolumn{8}{|c|}{ Strain assigned to cluster $46:$ Streptomyces pallidus (1) } \\
\hline 46 & $1-19$ & S. pallidus & DSM 40531 & ATCC 27457 ISP 5531 & A 18 & & \\
\hline \multicolumn{8}{|c|}{ Strain assigned to cluster $47:$ Streptomyces anulatus (1) } \\
\hline 47 & $1-35$ & S. anulatus \# & ${ }^{*} \operatorname{DSM} 40361^{\mathrm{T}}$ & ATCC 27416 ISP 5361 & A $1 B$ & Category I & Species no. 02 \\
\hline \multicolumn{8}{|c|}{ Strain assigned to cluster 48 : Streptomyces parvulus (1) } \\
\hline 48 & 26 & S. parvulus & *DSM 40722 & & & Category I & Species no. 07 \\
\hline \multicolumn{8}{|c|}{ Strains assigned to cluster $49:$ Streptomyces roseiscleroticus (2) } \\
\hline $\begin{array}{l}49 \\
49\end{array}$ & 22 & S. roseiscleroticus & ${ }^{*} \mathrm{DSM} 40303^{(\mathrm{T})}$ & ATCC 17755 ISP 5303 & & Category II & Species no. 19 \\
\hline 49 & 22 & S. ruber & ${ }^{*}$ DSM $40304^{(T)}$ & & & Category IV & Species no. 11 (red series) \\
\hline
\end{tabular}


Table 1 (continued)

\begin{tabular}{|c|c|c|c|c|c|}
\hline$s_{\text {SM }}{ }^{\dagger}$ & $S_{\mathrm{J}} \ddagger$ & Strain designation $\S$ & Strain numbers\| & $\begin{array}{l}\text { Williams } \\
\text { group }\end{array}$ & $\begin{array}{l}\text { Bergey category } \\
\text { and species no. } f \dagger\end{array}$ \\
\hline
\end{tabular}

$50 \quad 19$ S. saraceticus

$\begin{array}{lll}50 & 19 & S . \text { violatus }\end{array}$

$51 \quad 18 \quad$ S. antimycoticus

(Saccharothrix mutabilis)

$51 \quad 1-30 \quad S$. capreolus subsp. capreolus

$51 \quad 18$ S. melanosporofaciens

$51 \quad 18$ Streptomyces $\mathbf{s p}$

$51 \quad 18$ S. violaceusniger \#

52

17 S. bluensis

18 S. hygroscopicus

18 S. violaceusniger

18 S. violaceusniger

18 S. hygroscopicus

18 S. hygroscopicus

18 S. hygroscopicus

18 S. violaceusniger

18 S. violaceusniger

$55 \quad 18 \quad S$. hygroscopicus

$55 \quad 18$ S. rutgersensis subsp. castelarense

16 S. hygroscopicus subsp. ascomyceticus

$57 \quad 52 \quad$ S. hygroscopicus

$5753 \quad$ S. hygroscopicus

58

$50 S$. caeruleus

59

54 Streptomyces sp.

60

51 S. malachiticus

$61 \quad 1-22 \quad S$. bellus

63

$64 \quad 1-30 \quad S$. eburosporeus

64 1-30 'S. longissimus'

$64 \quad$ 1-30 Amycolatopsis mediterrane

$65 \quad 1-29 \quad$ Streptomyces sp.

$65 \quad 1-29$ Streptomyces sp.

$66 \quad 34$ S. alborubidus \#

$66 \quad 34$ S. albus

6634 S. flocculus

Strains assigned to cluster 50: Streptomyces violatus (2)

\begin{tabular}{|c|c|c|c|c|}
\hline $\begin{array}{l}\text { DSM } 40241 \\
{ }^{*} \text { DSM } 40209(T)\end{array}$ & $\begin{array}{l}\text { ATCC } 25496 \text { ISP } 5241 \\
\text { ATCC } 15892 \text { ISP } 5209\end{array}$ & $\begin{array}{l}\text { A } 29 \\
\text { A } 18\end{array}$ & Category I & Species no. 11 \\
\hline
\end{tabular}

Strains assigned to cluster 51: Streptomyces violaceusniger (5)

${ }^{*}$ DSM 40284 $\quad$ ATCC 23880 ISP 5284

*DSM 40225(T) ATCC 23892 ISP 5225

*DSM 40318(T) ATCC 25473 ISP 5318

DSM 40967

*DSM 40563 $\quad$ ATCC 27477 ISP 5563

J $\mathrm{Sm}$

A 32

A 32

Category IV Species no. 05 (grey series)

Strain assigned to cluster 52: Streptomyces bluensis (1)

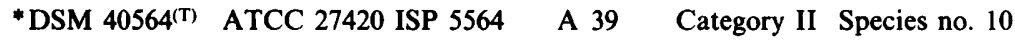

Strains assigned to cluster 53: Streptomyces violaceusniger (3)

$\begin{array}{llll}{ }^{*} \text { DSM } 41534 & \text { ATCC 31955 } & \text { Category I } & \text { Species no. 16 } \\ { }^{*} \text { DSM 41599 } & \text { NRRL B-1477 } & \text { Category I } & \text { Species no. 16 } \\ { }^{*} \text { DSM 41600 } & \text { NRRL B-1478 } & \text { Category I } & \text { Species no. 16 }\end{array}$

Strains assigned to cluster 54: Streptomyces violaceusniger (5)

*DSM 41524 ATCC $15166 \quad$ Category I Species no. 16

*DSM 41530 ATCC $29253 \quad$ Category I Species no. 16

*DSM 41603 NRRL B-1865 Category I Species no. 16

*DSM 41602 NRRL B-16257 Category I Species no. 16

*DSM 41598 NRRL B-1356 Category I Species no. 16

Strains assigned to cluster 55: Streptomyces hygroscopicus (2)

\begin{tabular}{|c|c|c|}
\hline "DSM 41535 & ATCC 39150 & Category I \\
\hline * DSM 40830 & ATCC 15191 & Category I \\
\hline
\end{tabular}

Strain assigned to cluster 56: Streptomyces hygroscopicus (1)

DSM 40822 ATCC 14891

Strains assigned to cluster 57: Streptomyces hygroscopicus (2)

*DSM 41533 ATCC $31080 \quad$ Category I Species no. 16

*DSM 41529 ATCC $21840 \quad$ Category I Species no. 16

Strain assigned to cluster 58: Streptomyces caeruleus (1)

${ }^{*}$ DSM 40103 $\quad$ ATCC 27421 ISP 5103

Category IV Species no. 07 (grey series)

Strain assigned to cluster 59: Streptomyces sp. (1) DSM 40966

Strain assigned to cluster 60: Streptomyces malachiticus (1) DSM 40167 ATCC 19918 ISP 5167 A 12

Strain assigned to cluster 61 : Streptomyces bellus (1)

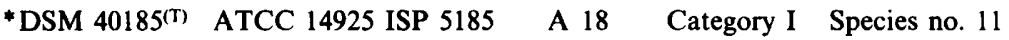

Strain assigned to cluster 62: Streptomyces xantholiticus (1)

${ }^{*}$ DSM 40244(T) ATCC 27481 ISP 5244 C 24 Category II Species no. 05

Strain assigned to cluster 63: Streptomyces albosporeus (1)

*DSM 40795T ATCC 15394

Category IV Species no. 01 (red series)

Strains assigned to cluster 64: 'Streptomyces longissimus' (3)

DSM 40944

DSM 40826

*DSM 40773

ATCC 14562

Strains assigned to cluster 65: Streptomyces sp. (2)

DSM 40988

DSM 40986

Strains assigned to cluster 66: Streptomyces flocculus (3)

DSM 40465 ATCC 23612 ISP 5465 A 12

*DSM 40785

*DSM 40936
Category I Species no. 09

Category I Species no. 09 
Table 1 (continued)

\begin{tabular}{|c|c|c|c|c|c|c|}
\hline$S_{\mathrm{SM}} \dagger$ & $S_{\mathrm{J}} \ddagger$ & Strain designation $\S$ & Stra & ain numbers $\|$ & $\begin{array}{l}\text { Williams } \\
\text { group }\end{array}$ & $\begin{array}{l}\text { Bergey category } \\
\text { and species no. }+\dagger\end{array}$ \\
\hline \multicolumn{7}{|c|}{ Strain assigned to cluster 67 : Nocardia sp. (1) } \\
\hline 67 & 34 & Nocardia sp. (S. listeri) & DSM 40297 & & & \\
\hline \multicolumn{7}{|c|}{ Strains assigned to cluster 68 : Streptomyces sulphureus (2) } \\
\hline $\begin{array}{l}68 \\
68\end{array}$ & $\begin{array}{l}2 \\
2\end{array}$ & $\begin{array}{l}\text { Streptomyces sp. } \\
\text { S. sulphureus }\end{array}$ & $\begin{array}{l}\text { DSM } 40968 \\
{ }^{*} \text { DSM } 40104^{T}\end{array}$ & ATCC 27468 ISP 5104 & C Sm & Category III Species no. 17 \\
\hline \multicolumn{7}{|c|}{ Strains assigned to cluster $69:$ Streptomyces ochraceiscleroticus (10) } \\
\hline 69 & $1-26$ & $S$. niger & * DSM $40302^{(\mathrm{T})}$ & & A 40 & Category I Species no. 18 \\
\hline 69 & $1-26$ & S. ochraceiscleroticus & DSM 40594 & & & Category III Species no. 08 \\
\hline 69 & $1-26$ & S. ochraceiscleroticus & ${ }^{*} \mathrm{DSM} 43155^{(\mathrm{T})}$ & & & Category III Species no. 08 \\
\hline 69 & $1-26$ & S. olivaceiscleroticus & ${ }^{*} \mathrm{DSM} 40595^{\mathrm{T}}$ & ATCC 15722 ISP 5595 & & Category IV Species no. 24 (grey series) \\
\hline 69 & $1-26$ & S. purpurogeneiscleroticus & *DSM $40271^{(\mathrm{T})}$ & & A 40 & Category I Species no. 18 \\
\hline 69 & $1-26$ & S. sclerotialus & ${ }^{*}$ DSM $40269^{(T)}$ & & & Species no. 18 \\
\hline 69 & $1-26$ & Streptomyces sp. & DSM 40156 & & & \\
\hline 69 & $1-26$ & S. verne & DSM 40079 & ATCC 3353 ISP 5079 & A 40 & \\
\hline 69 & $1-26$ & S. violaceoruber & ${ }^{*}$ DSM $40049^{\mathrm{T}}$ & ATCC 14980 ISP 5049 & & Category IV Species no. 34 (grey series) \\
\hline 69 & $1-26$ & S. violens & ${ }^{*} \mathrm{DSM} 40597^{(\mathrm{T})}$ & ATCC 15898 ISP 5597 & A 40 & Category I Species no. 18 \\
\hline \multicolumn{7}{|c|}{ Strain assigned to cluster $70:$ Streptomyces fradiae (1) } \\
\hline 70 & $1-26$ & S. fradiae & *DSM 40943 & & & Category II Species no. 18 \\
\hline \multicolumn{7}{|c|}{ Strain assigned to cluster $71:$ Streptomyces poonensis (1) } \\
\hline 71 & $1-19$ & S. poonensis & ${ }^{*}$ DSM $40596^{(T)}$ & ATCC 15723 ISP 5596 & A 22 & Category II Species no. 19 \\
\hline \multicolumn{7}{|c|}{ Strain assigned to cluster $72:$ Streptomyces sp. (1) } \\
\hline 72 & $1-26$ & Streptomyces sp. & DSM 40857 & ATCC 11862 & & \\
\hline \multicolumn{7}{|c|}{ Strain assigned to cluster 73: Streptomyces olivaceus (1) } \\
\hline 73 & $1-26$ & S. olivaceus & *DSM 40756 & ATCC 11071 & & Category I Species no. 03 \\
\hline \multicolumn{7}{|c|}{ Strain assigned to cluster $74:$ Streptomyces erythrogriseus (1) } \\
\hline 74 & $1-27$ & S. erythrogriseus & ${ }^{*}$ DSM $40116^{\mathrm{T}}$ & ATCC 27427 ISP 5116 & & Category IV Species no. 04 (red series) \\
\hline \multicolumn{7}{|c|}{ Strain assigned to cluster $75:$ Streptomyces bambergiensis (1) } \\
\hline 75 & $1-25$ & S. bambergiensis & ${ }^{*}$ DSM $40590^{\mathrm{T}}$ & ATCC 13879 ISP 5590 & A Sm & Category III Species no. 10 \\
\hline \multicolumn{7}{|c|}{ Strains assigned to cluster $76:$ Streptomyces $\mathrm{sp} . /$ Streptoverticillium $\mathrm{sp} . /$ Nocardia $\mathrm{sp}$. (9) } \\
\hline 76 & 73 & N. carneus & DSM 40840 & & & \\
\hline 76 & 72 & N. coroniformis & DSM 40806 & & & \\
\hline 76 & 69 & S. cinnerocrocatus & DSM 40876 & & & \\
\hline 76 & 69 & S. galtieri (N. galtieri) & DSM 40350 & ATCC 27433 & & \\
\hline 76 & 71 & S. phaeochromogenes & * DSM 40788 & NRRL B-1266 & & Species no. 18 \\
\hline 76 & 71 & S. rubescens & DSM 40777 & NRRL B-1519 & & \\
\hline 76 & 70 & Nocarida salmonicida & DSM 40472 & & I 69 & \\
\hline 76 & 69 & S. viridis & DSM 40637 & & & \\
\hline 76 & 69 & Sv. albireticuli & ${ }^{*} \mathrm{DSM} 40051^{\mathrm{T}}$ & ATCC 19721 ISP 5051 & F SM & Species no. 11 \\
\hline \multicolumn{7}{|c|}{ Strain assigned to cluster $77:$ Streptomyces ipomoeae (1) } \\
\hline 77 & 74 & S. ipomoeae & ${ }^{*} \mathrm{DSM} 40383^{\mathrm{T}}$ & ATCC 25462 ISP 5383 & & Category IV Species no. 02 (blue series) \\
\hline \multicolumn{7}{|c|}{ Strains assigned to cluster $78:$ Streptomyces fragilis (2) } \\
\hline 78 & 58 & S. fragilis & ${ }^{*} \mathrm{DSM} 40044^{\mathrm{T}}$ & ATCC 23908 ISP 5044 & G SM & Category III Species no. 22 \\
\hline 78 & 60 & S. griseoplanus & ${ }^{*}$ DSM $40009^{(\mathrm{T})}$ & ATCC 19766 ISP 5009 & A 29 & Category I Species no. 15 \\
\hline \multicolumn{7}{|c|}{ Strains assigned to cluster 79 : Streptomyces lipmanii (3) } \\
\hline 79 & 63 & S. amakusaensis & ${ }^{*}$ DSM $40219^{\mathrm{T}}$ & ATCC 23876 ISP 5219 & B Sm & Category III Species no. 12 \\
\hline 79 & 61 & S. lipmanii & *DSM 40752 & ATCC 27357 & & Category I Species no. 02 \\
\hline 79 & 44 & S. olivaceus & *DSM 41541 & ATCC 21542 & & Category I Species no. 03 \\
\hline \multicolumn{7}{|c|}{ Strains assigned to cluster $80:$ Streptomyces sp. (3) } \\
\hline 80 & 65 & S. fradiae & *DSM 41547 & ATCC 21096 & & Category II Species no. 18 \\
\hline 80 & 64 & S. glaucescens & * DSM 40510 & & & Category III Species no. 05 \\
\hline 80 & 66 & S. verevaensis & ${ }^{*}$ DSM $43167^{\mathrm{T}}$ & & & Category III Species no. 18 \\
\hline \multicolumn{7}{|c|}{ Strain assigned to cluster $81:$ Streptomyces exfoliatus (1) } \\
\hline 81 & 55 & S. exfoliatus & ${ }^{*}$ DSM 40735 & & & Species no. 04 \\
\hline \multicolumn{7}{|c|}{ Strains assigned to cluster $82:$ Streptomyces sp. (2) } \\
\hline 82 & 77 & S. chrysomallus & *DSM 40685 & & & \\
\hline 82 & 78 & Streptomyces sp. & DSM 40889 & & & \\
\hline
\end{tabular}


Table 1 (continued)

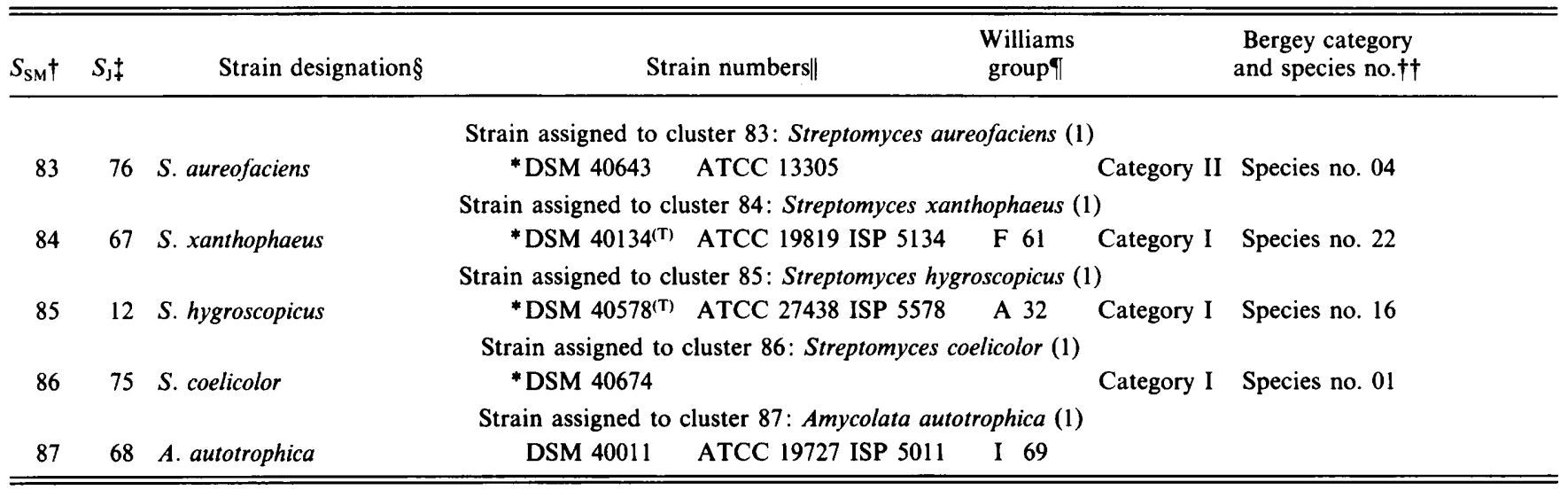

$\dagger$ Number of cluster (and subcluster) in the UPGMA $/ S_{\mathrm{SM}}$ analysis.

$\ddagger$ Number of cluster (and subcluster) in the UPGMA $/ S_{\mathrm{J}}$ analysis (numbers of strains for each cluster and subcluster are given in the text).

$\S$ Designation of strain as received from the German Collection of Micro-organisms (DSM). The 20 strains included in the test reproducibility study are marked \#.

\| Strains are marked with an asterisk when the species name is listed in the Approved Lists of Bacterial Names (Skerman et al., 1980). A superscript $T$ denotes the type strain of the species according to Williams et al. (1989); a superscript (T) denotes the type strain of the nomenspecies in the Approved Lists. Abbreviations of culture collections, etc.: ATCC, American Type Culture Collection, Rockville, MD, USA: DSM, Deutsche Sammlung von Mikroorganismen und Zellkulturen, Braunschweig, FRG; IFO, Institute for Fermentation, Osaka, Japan; ISP, International Streptomyces Project; IMRU, Institute of Microbiology, Rutgers-State University, NJ, USA; NCIB, National Collection of Industrial Bacteria, Aberdeen, UK; NRRL, Northern Utilization Research and Development Division, Peoria, IL, USA.

I Cluster-group and number of the numerical analysis of Williams et al. (1983a).

$\dagger \dagger$ Assignment of strains to species according to Bergey's Manual of Systematic Bacteriology, vol. 4. For the genus Streptomyces, species categories I to IV are shown, with the species number within the category according to Williams et al. (1989); for species category IV the 'series' assignment is also given, according to spore colour. For the genus Streptoverticillium, species numbers are according to Locci \& Schofield (1989).

Williams et al. (1983a), the name proposed by these authors was chosen for the phenon.

The following description of the phena is presented in comparison with the study of Williams et al. (1983a) and the resulting species descriptions in Bergey's Manual of Systematic Bacteriology, vol. 4 (Williams et al., 1989). The clusters of Williams et al. (1983a) are in places referred to as 'Williams groups', to avoid confusion with the clusters obtained in the present study. The three categories of the spore chains, viz. (a) straight to flexuous (Rectiflexibilis; RF), (b) hooks, loops or spirals with one to two turns (Retinaculiaperti; RA), and (c) spirals (Spirales; SP), are also given, although it is generally accepted that more than one category can be observed in the same species and the distinction between Retinaculiaperti and Spirales is not clear (Williams \& Wellington, 1980; Williams et al., 1989). The colour of the spore mass is also given, although its determination is not always easy (Kutzner, 1981), but 'species' assignment to different series within category IV (Williams et al., 1989) is still based on these morphological properties. Physiological characters most representative of each cluster are marked in Table 2.

\section{Composition and characteristics of the major clusters}

Cluster 1 (S. albidoflavus/S. griseus/S. antibioticus) was the largest cluster of the analysis and contained 206 strains. The majority of these strains formed RF or RA spore chains with smooth, white, grey or yellow spores. It could be divided into eight subclusters (1-1 to 1-8) at the $84.0 \%$ similarity level $\left(S_{\mathrm{SM}}\right)$.

Subcluster 1-1 (S. albidoflavus) contained 52 strains, the majority of them from Williams cluster group A1A (S. albidoflavus). Additionally, eight strains were found in this cluster which were not tested by Williams et al. (1983a), but which belong to this species (Williams et al., 1989). This subcluster also contained strain ISP 5139 ( $S$. badius) from Williams cluster group $C$ (single-member cluster), strain ISP 5557 (S. alni) from group A8, strain ISP 5335 (S. prunicolor) from group A11 and strain ISP 5496 ( $S$. diastaticus) from group A 19. In this cluster, three strains of $S$. albus were found, which proved to be wrongly designated. The cluster contained in addition $S$. somaliensis strain ISP 5267 which belongs to the yellow series according to Shirling \& Gottlieb, $1968 b$. All strains formed smooth, yellow or white spores in RF chains. 


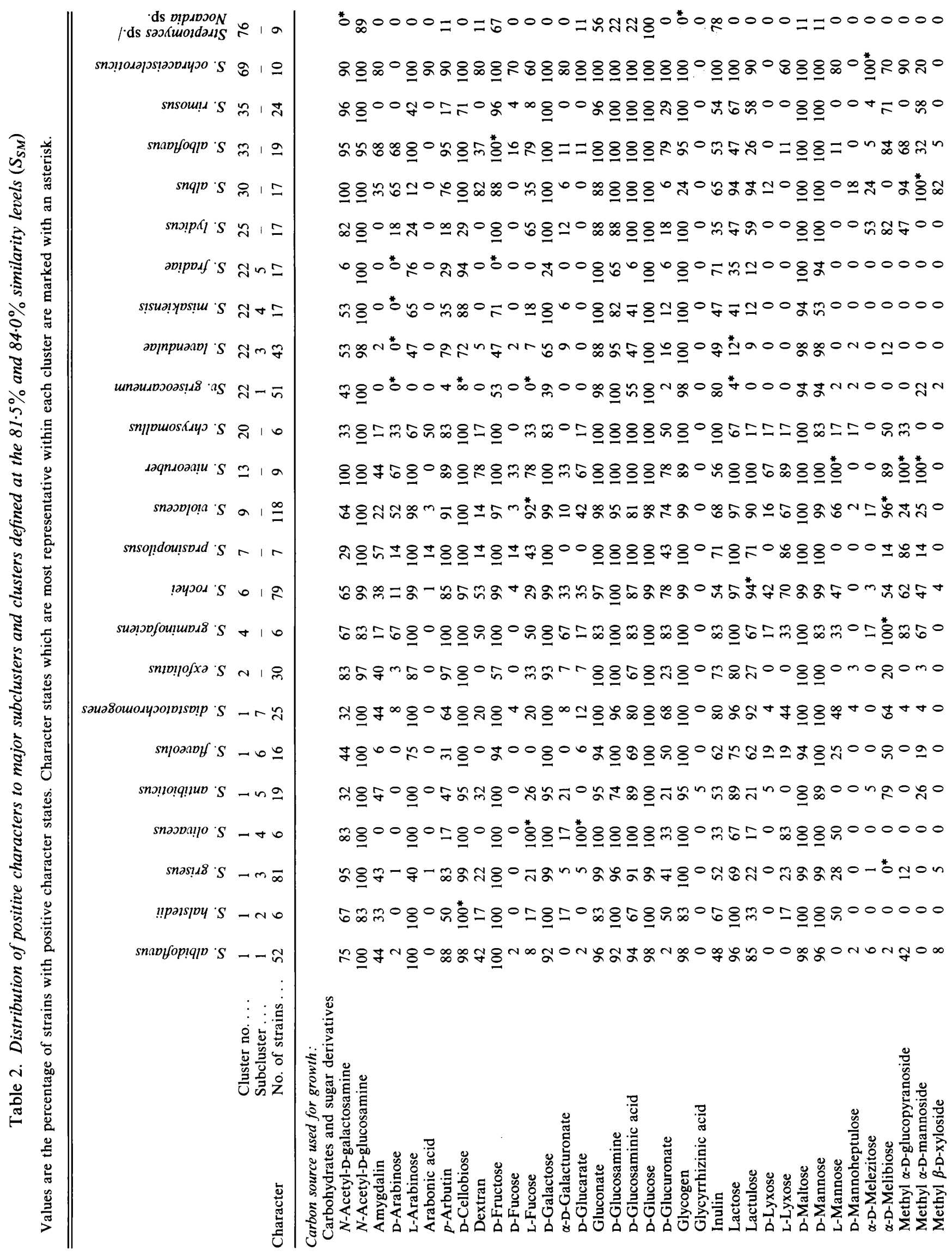




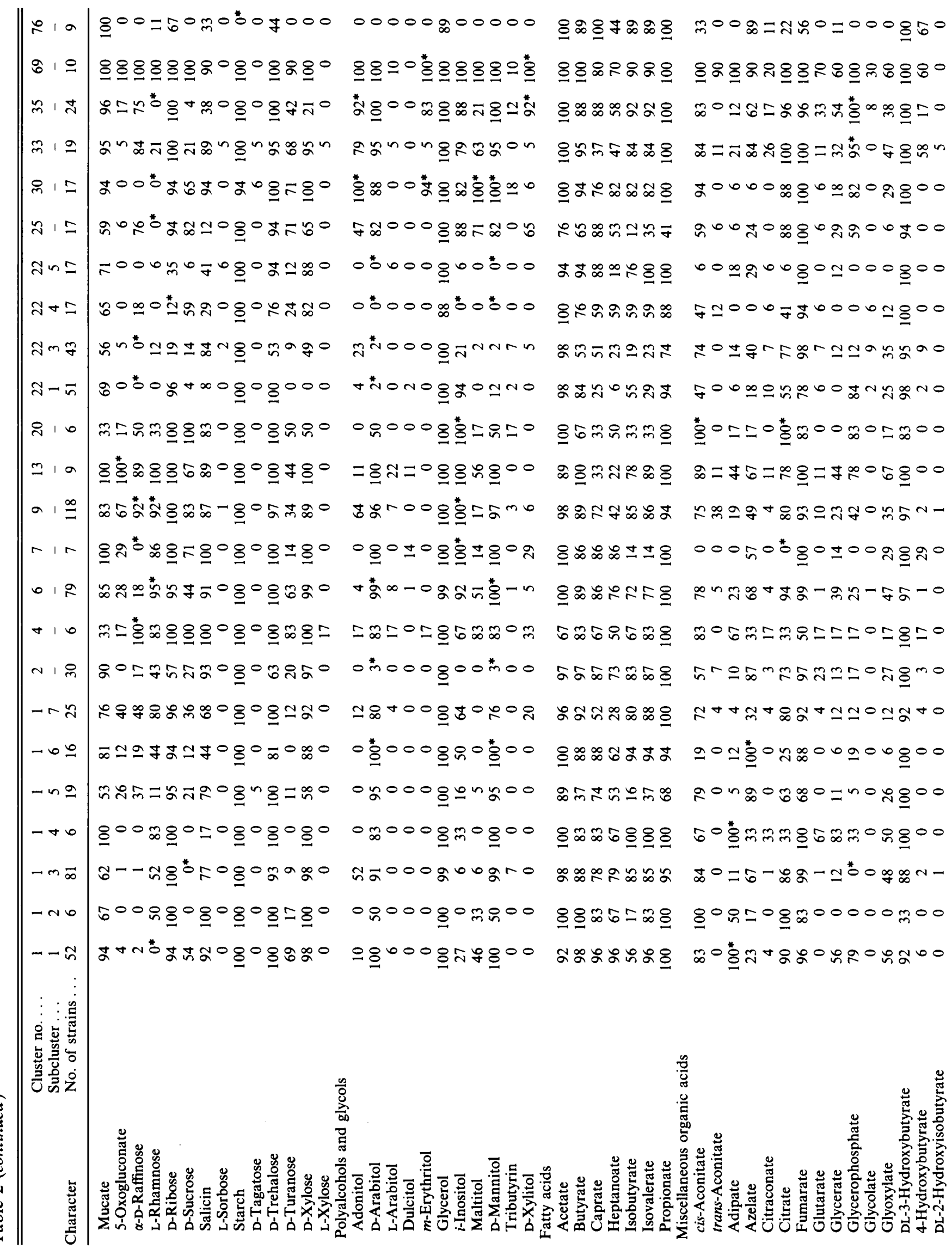




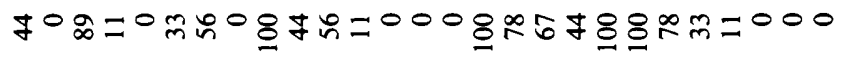

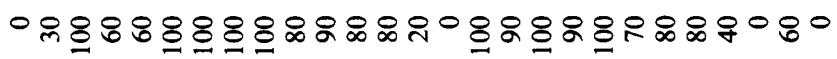
ニ ณ

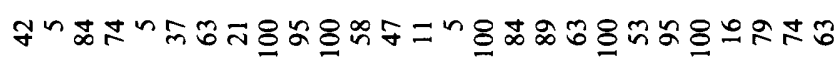

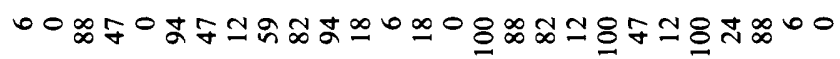

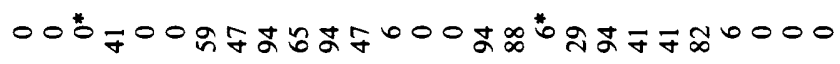

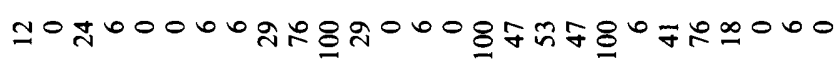

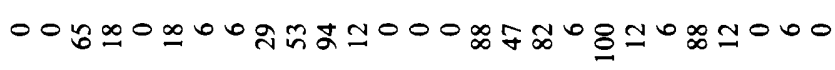

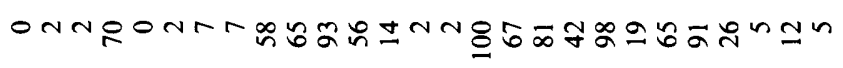

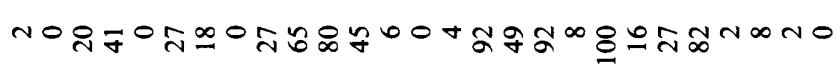

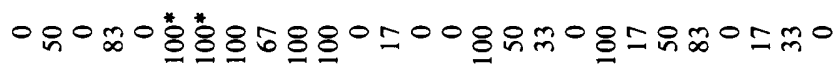
ニニチレ゚

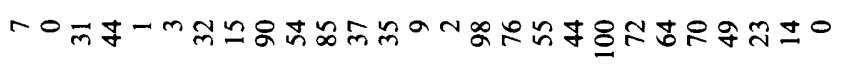

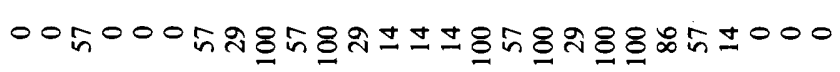

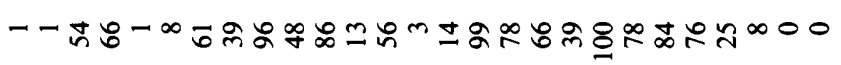

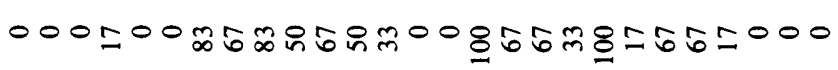

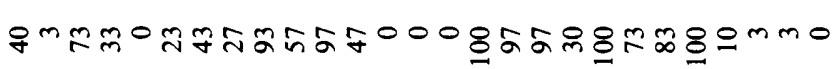
○ +

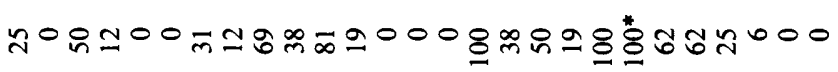

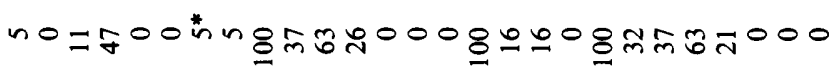

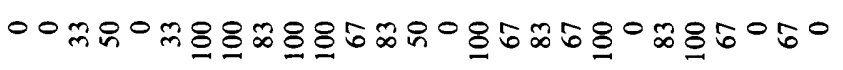

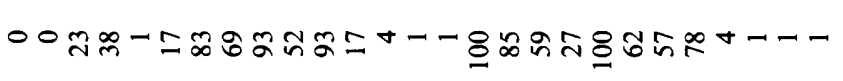

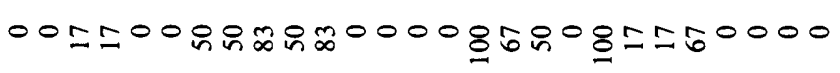

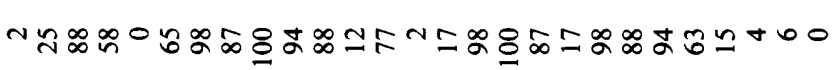

$=0408 \quad t=000004=000000000=4 \pi 0$

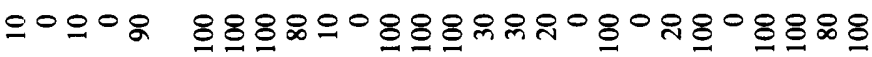

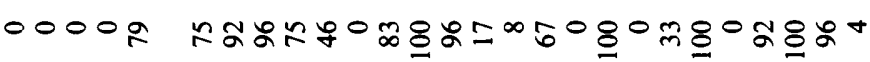

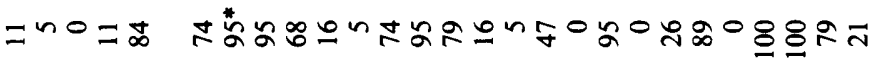
0000 ถู

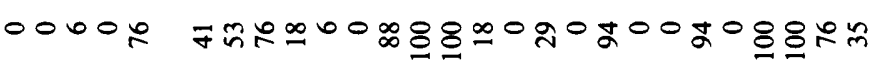
ด 00 กิ 0000 ㄱ ำกำ o

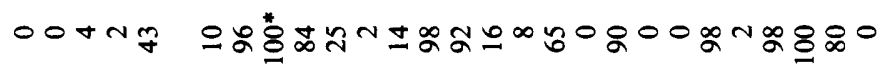

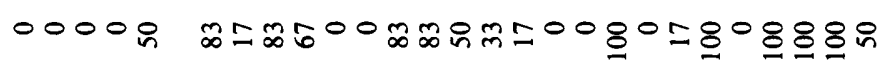

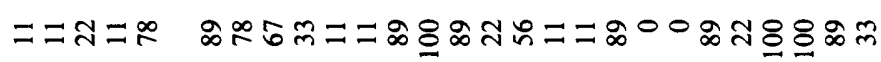

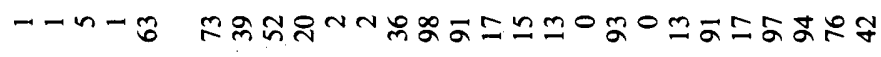
OOJ依

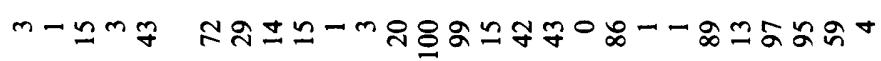

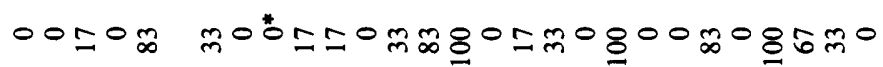

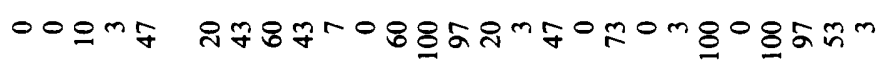

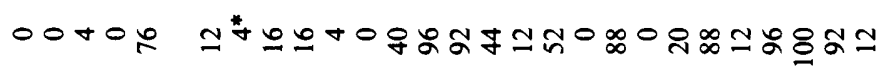

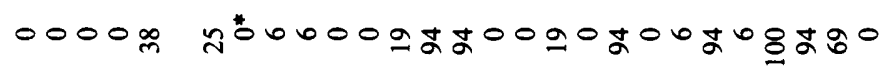

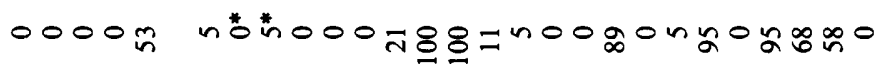
0000 o ONOM०

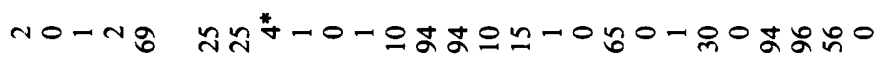

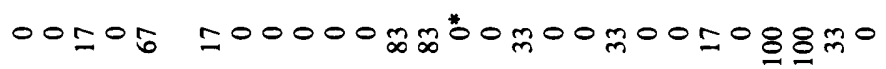

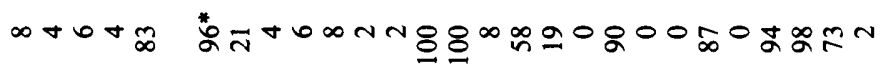

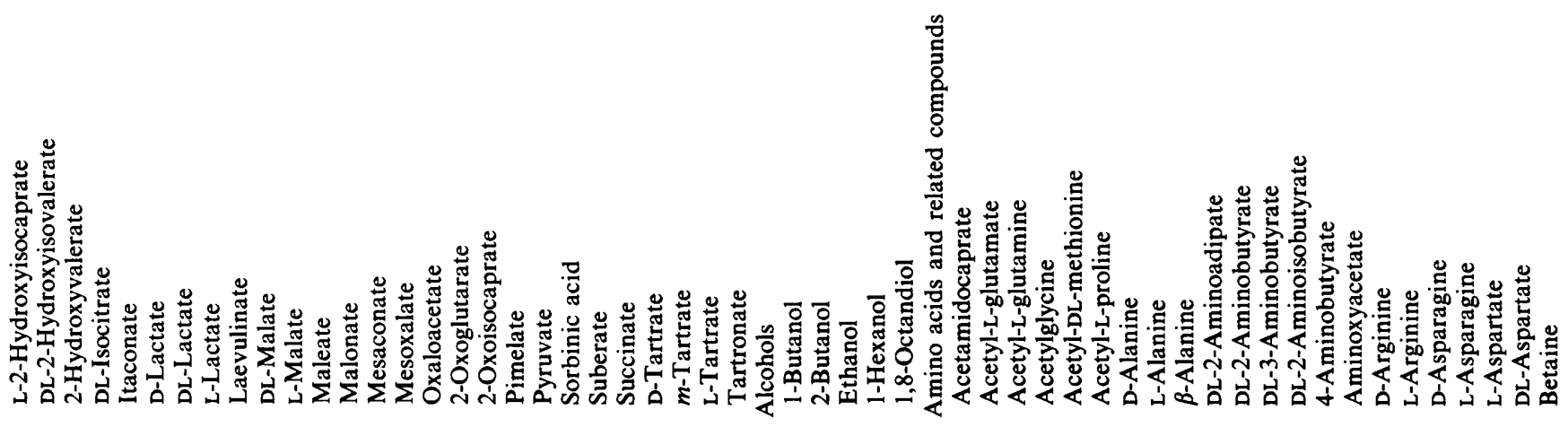




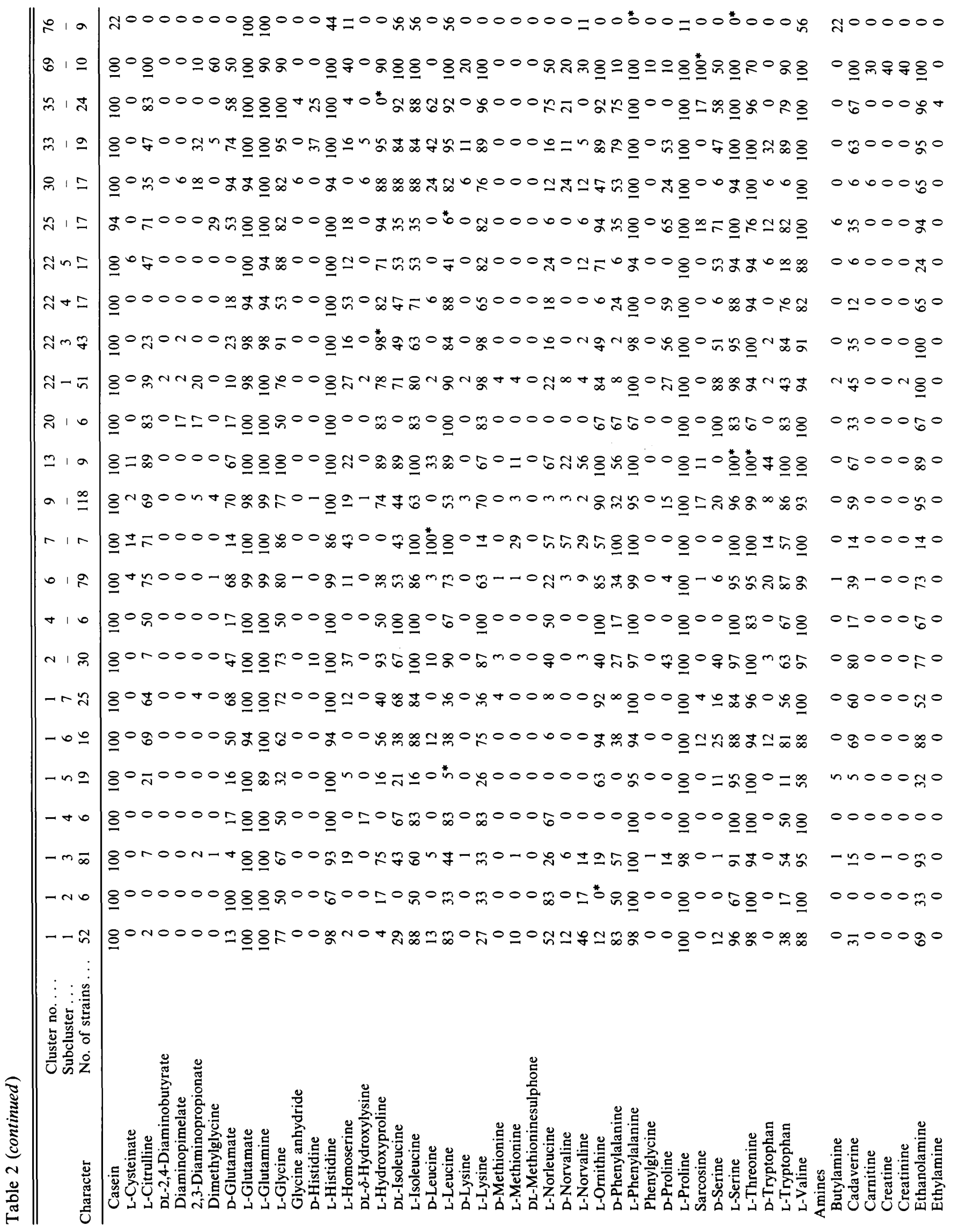


$0 \mathscr{R}^{\circ}=00000=0=0=0$ ஜ $00000 \mathrm{~N}$

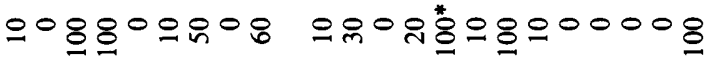
용ํㅇㅇㅏ $008 . \overline{0} 0000$ $008 \infty 00000$ $008 \pi 0000$ $008^{*}$ 이잉

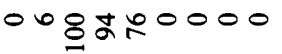
nne87000

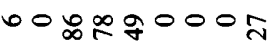
$00 \%=0000$ $=0820000=$ +ogano+o m०ะ800000 moggoo$0088=0000$ - 080000 N

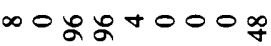
$00 \pm 80000 \%$ $0088=000 \mathrm{~m}$ 005800000 r 요요잉

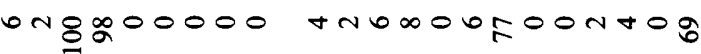

$000=0$ \%

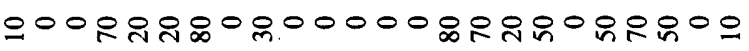

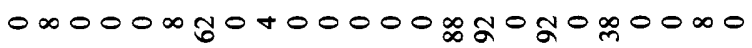

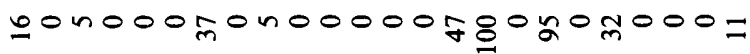

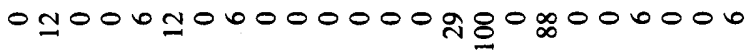

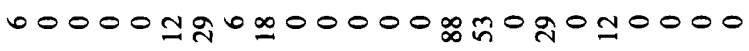
00000 N00000000MNOFONO000 N0000000000000 nूn

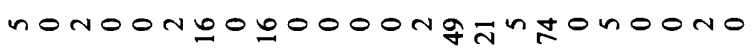

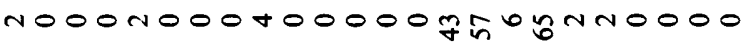
IOOOOINON00000\%

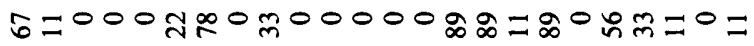
エー

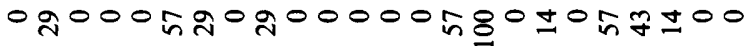

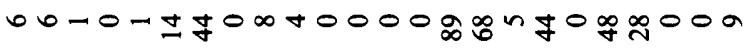

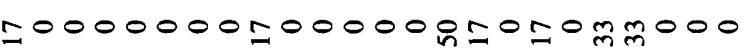

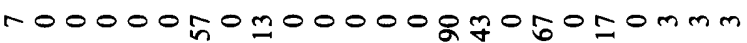
OHOOHDODODOOONMN

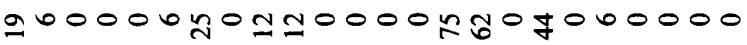
noO $00000000=000008808000000$

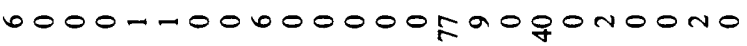
$00000 m 00000000 m=00000000$

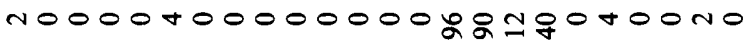

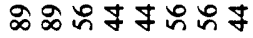
88888ஃஃ: $888889 \approx \infty$

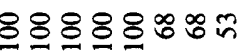
88888スㅇ

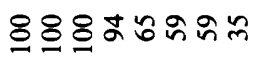
8옳ำกำ 응ำำ ำ 오ำษ 8ㅇำஸัก

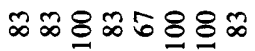
8888뮤

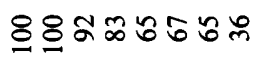
8ㅇㅇㅇ요호

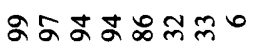
88555 ํํㅇ 오ํㅇㅇㅇㅛ

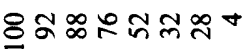

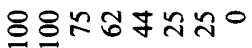

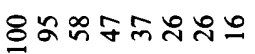

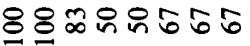

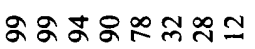

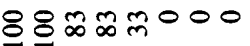

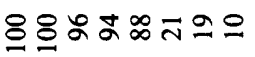

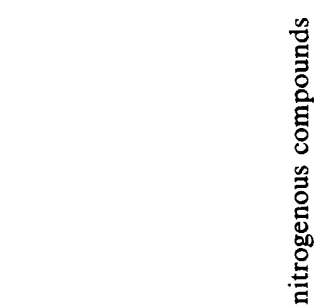

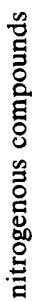
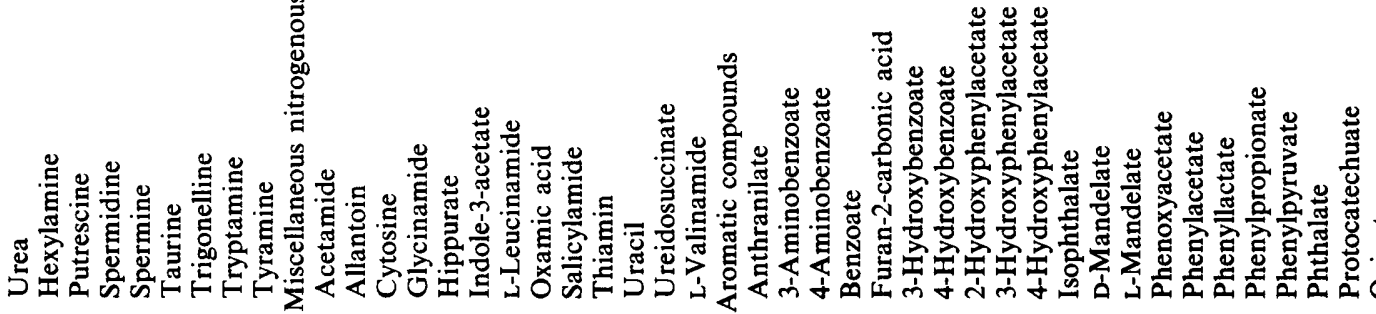
สำ $=0000=\infty .800=40=8$

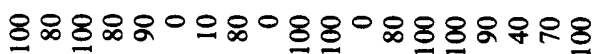

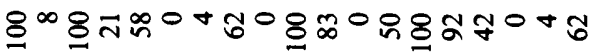

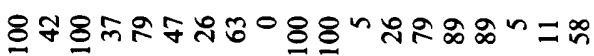

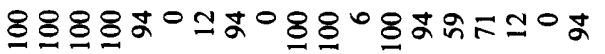

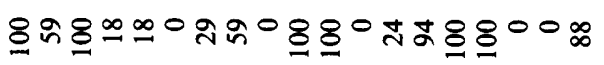

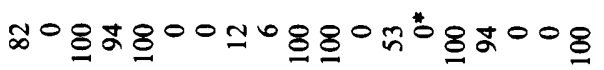

К음ำ

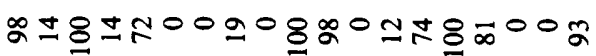

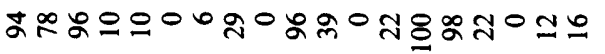

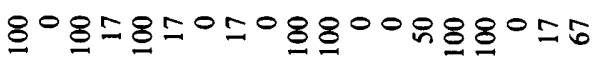

कి

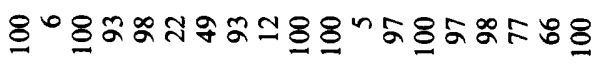

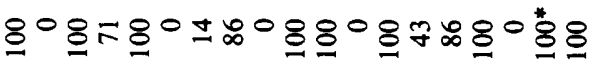

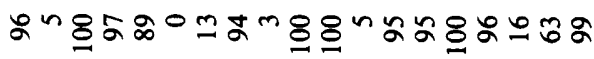

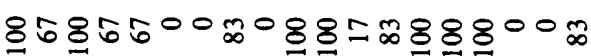

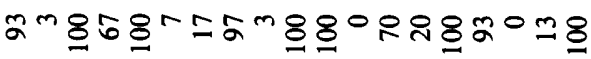

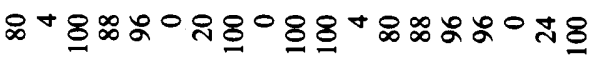

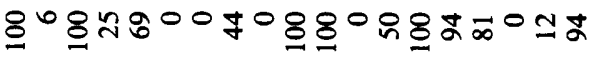

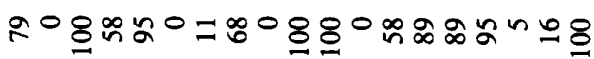

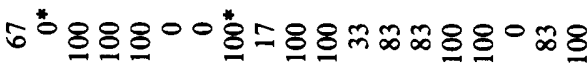

8-

mo8.80050880=188008

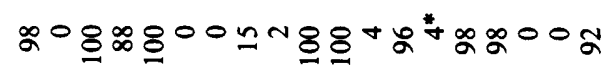

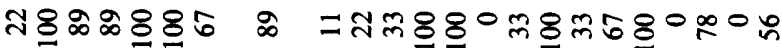

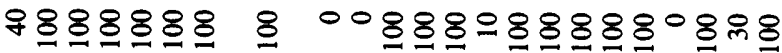

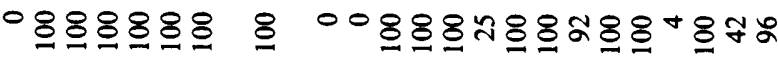

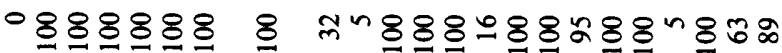

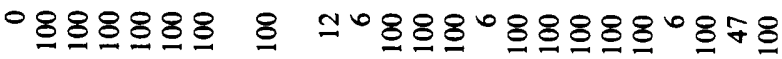

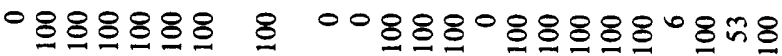

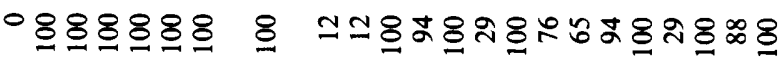

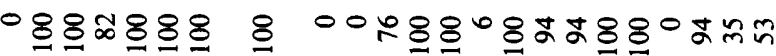

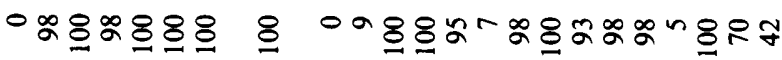

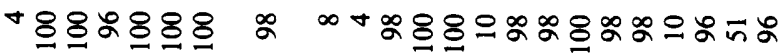

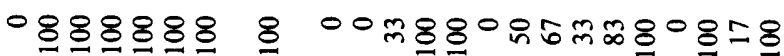

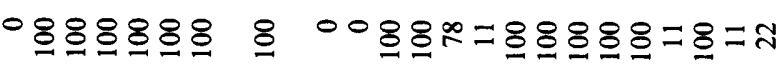

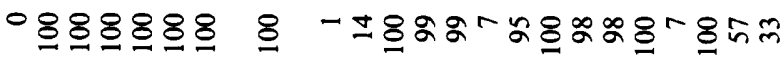

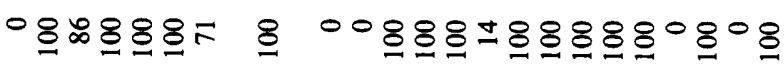

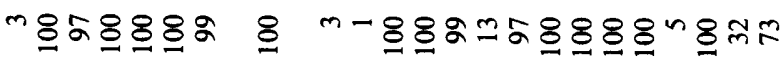

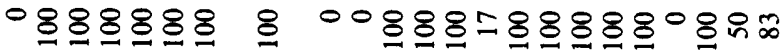

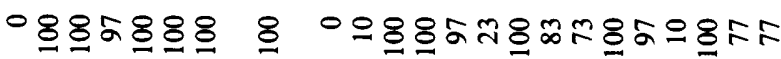

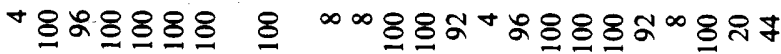

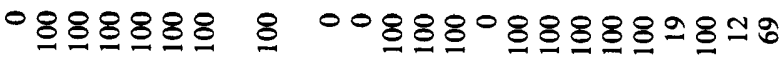

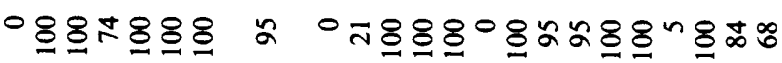
-

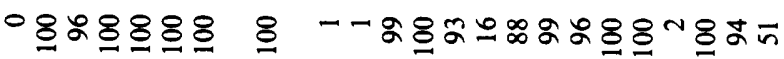

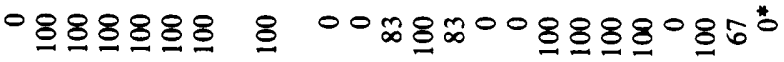

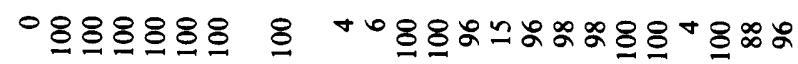

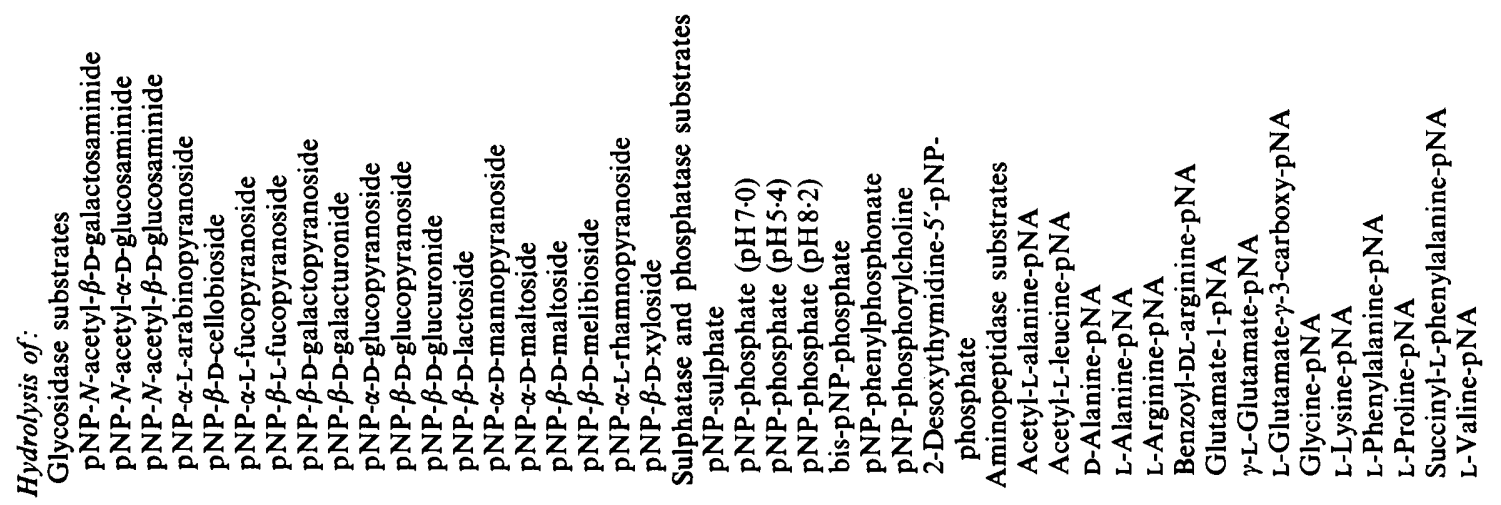


$=00000 \mathrm{H}=00 \stackrel{x}{=0}=0000000000000000 \mathrm{Z}=0=00000$ 00500000

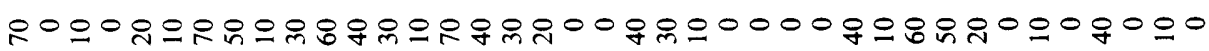
은유으융이 motomon

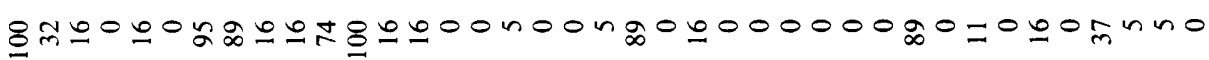
Foo

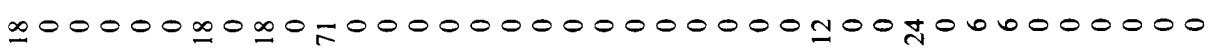
AONO000000MINIOONO000000000000000000000

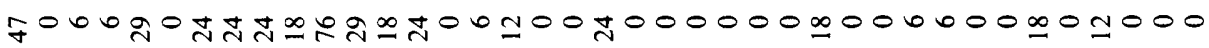

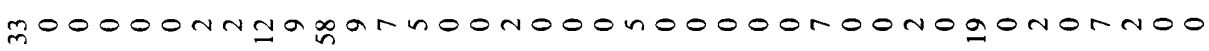

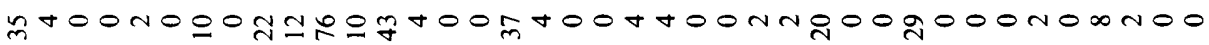
$00000=008=80000000000000008000000000000$

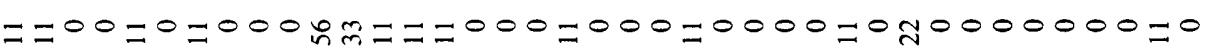

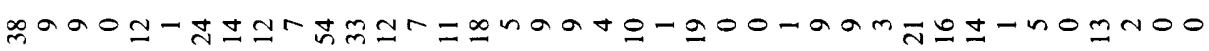
o

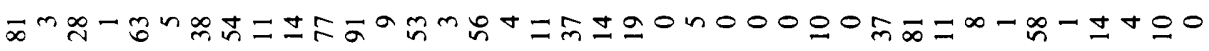
NOEOOONORIm000NON000000000m00m000000000

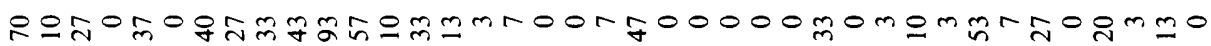

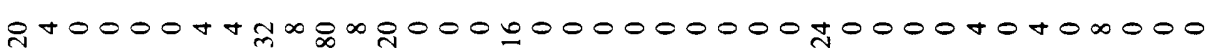

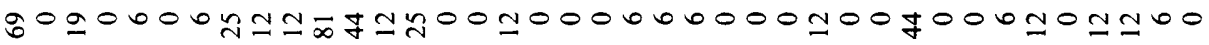

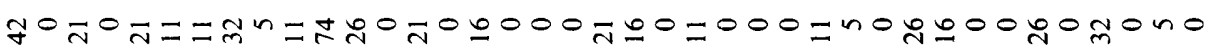
$m O m O E O=000 m=00000000000000000 m 000000000$ motonoghan

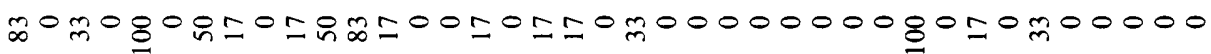
Ang

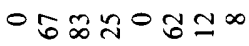
o ononooo

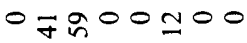
00 mo0000 00 m00000 00900000 00 ño00 N noknOOnN 00000000 $0080000=$ $0-\pi-0 m-1$ - mo0 o 00 m00000 oomoommm $00 \% 0000 \%$ $00 n 00000$ o o promoo 00100000 ogoonon 00800000 Oก กONOO

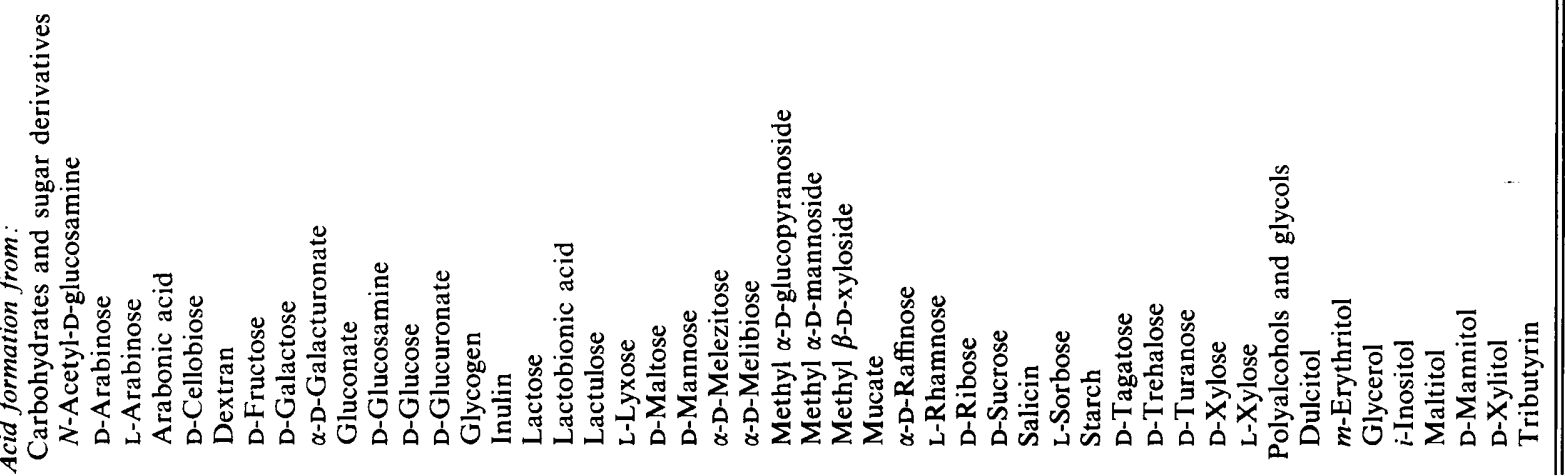


Subcluster 1-2 ( $S$. halstedii) consisted of six strains. Five of these belonged to Williams group A1C ( $S$. halstedii); the other strain, DSM 40859 (S. scabies), is probably wrongly designated. All strains of this cluster formed smooth, grey spores in RF chains.

Subcluster 1-3 (S. griseus) contained 81 strains, the majority of them belonging to Williams group A1B ( $S$. anulatus). This cluster also contained one strain each from the Williams clusters A6, A9 and A23, and $S$. floridae and $S$. praecox (category IV, yellow series), in addition to three strains grouped into cluster A3. These strains shared the morphological properties of the majority of the strains in subcluster 1-3 (yellow spore mass, smooth spores in RF chains). $S$. acrimycini (category IV, green series) was also recovered in this subcluster, despite its morphological characteristics (green spores with a hairy surface, SP or RA spore chains). In addition to these strains in subcluster 1-3, two strains of $S$. fradiae and two strains of $S$. lavendulae were found which were obviously wrongly designated. Subcluster 1-3 also contained strain ISP 5078, designated as S. scabies and grouped into cluster A3 of Williams et al. (1983a) but not included in the revival of $S$. scabies by Lambert \& Loria (1989a). S. setonii ATCC 25497, which was considered to be a strain of $S$. griseus by Hütter (1967), also fell within this subcluster. Strain $S$. anulatus ISP 5361, responsible for the name of the corresponding cluster A1B ( $S$. anulatus) of Williams et al. (1983a) formed single-member cluster 47 in the present analysis.

Subcluster 1-4 ( $S$. olivaceus) consisted of six strains, four of them named $S$. olivaceus and all sharing the morphological properties of strains grouped into subcluster 1-2. In addition, one strain named $S$. scabies (DSM 40611) was found in this subcluster.

The four subclusters 1-1 to 1-4 were joined at the $82.3 \%$ similarity level $\left.S_{\mathrm{SM}}\right)$.

Subcluster 1-5 (S. antibioticus) contained 19 strains, most of them received as $S$. antibioticus. All strains formed grey spores; the majority had a smooth surface. Spore chains were RF, RA or SP. Williams et al. (1983a) grouped five strains into a heterogeneous cluster (A31) named $S$. antibioticus. Only one strain (S. lucensis, ISP 5317) of this Williams cluster was recovered in subcluster 1-5. However, two further strains from Williams cluster A31 were found in subcluster 1-6 ( $S$. flaveolus), thus confirming the heterogeneity of the species. Furthermore, all three strains of Williams group A34 ( $S$. nogalater) which were later classified into different species (Williams et al., 1989) were found in subcluster 1-5. The strain named ' $S$. lavenduligriseus' ISP 5487 is classified in the genus Streptoverticillium (Sv. lavenduligriseum) acccording to Locci \& Schofield (1989). The subcluster contained further strains from different Williams groups which are listed in detail in Table 1.
Subcluster 1-6 ( $S$. flaveolus) was composed of 16 strains, 10 of them tested by Williams et al. (1983a) and grouped into clusters A1B, A12, A17, A18, A24, A 31 and A33. All strains, with the exception of $S$. alboniger (ISP 5043) produced grey or red, smooth or hairy spores in SP or RF spore chains.

Subcluster 1-7 ( $S$. diastatochromogenes) contained 25 strains. Three of these were strains from Williams group A19 ( $S$. diastaticus) which were not grouped into our cluster 9 containing the majority of strains from A19. Besides these strains, members of the Williams groups $\mathrm{A} 12, \mathrm{~A} 18, \mathrm{~A} 23$ and A31, S. bobili (ISP 5056) of category IV, white series, and $S$. ghanaensis (DSM 40746), green series (Williams et al., 1989) were also found. Three strains of $S$. glaucescens not tested by these workers were additionally recovered in this subcluster. Most strains had the morphological properties found in other subclusters of cluster 1 , but this subcluster had no corresponding cluster in the study of Williams et al. (1983a).

Subcluster 1-8 ('Sv. luteoreticuli') contained only strain ISP 5509, which was classified as $S v$. mobaraense by Locci \& Schofield (1989).

The subclusters $1-5$ to $1-8$ were joined at the $83 \% S_{\mathrm{SM}}$ similarity level.

Cluster 2 (S. exfoliatus) was composed of 30 strains. Of these, 12 strains belonged to Williams group A5 ( $S$. exfoliatus) and three to the strongly related Williams group A6 ( $S$. violaceus), in addition to one strain of Williams group A7. All strains, including strain $S$. tanashiensis ISP 5195 (category IV, grey series of Williams et al., 1989) produced RF chains of smooth, red, grey or white spores.

Cluster 4 ( $S$. graminofaciens) was composed of six strains and contained both the strains from Williams group A26 ( $S$. graminofaciens) in addition to strains from different Williams groups. It has no corresponding cluster in the study of Williams et al. (1983a).

Cluster 6 (S. rochei) was composed of 79 strains, the majority of them belonging to Williams groups A12 ( $S$. rochei) and A21 (S. griseoruber). Single strains from the Williams groups A1C, A13, A15, A37, A38 and C44 were also recovered. This cluster also contained ten strains from category IV (Williams et al., 1989): eight strains of the grey series, one strain of the red series ( $S$. rubiginosohelvolus ISP 5176) and one strain of the green series ( $S$. griseostramineus ISP 5161). Most strains produced SP or RF chains of smooth grey spores, and produced no melanin or other pigments.

Cluster 7 ( $S$. prasinopilosus) consisted of seven strains, three of them classified in cluster A37 (S. griseoflavus) of Williams et al., 1983a). They produced ornamented, green spores in RA or SP chains and were melanin negative. 
Cluster 9 (S. violaceus) was the third-biggest cluster of the $S_{\mathrm{SM}}$ analysis, containing 118 strains. Most of these strains were classified into clusters A18 ( $S$. cyaneus) and A19 (S. diastaticus) of Williams et al. (1983a). Furthermore, the cluster contained all four strains of cluster A30 (S. filipinensis), three of the five strains of cluster A33 ( $S$. chromogenus), many strains designated as $S$. viridochromogenes and $S$. chartreusis, and six strains of category IV, three belonging to the grey series, two belonging to the blue series and one strain belonging to the red series (Williams et al., 1989). The cluster contained many strains producing blue, spiny spores ( $S$. chartreusis). Other strains produced grey or red spores in RF or SP chains.

Cluster 13 (S. niveoruber) was composed of nine strains, which produced grey, smooth spores in SP or RF chains, and of which only one ( $S$. toyocaensis ISP 5030) was tested by Williams et al. (1983a) and grouped into cluster A29. Three of the strains in cluster 13 are currently classified within category IV of Williams et al. (1989).

Cluster 20 (S. chrysomallus) was composed of six strains, none of them included in the study of Williams $e t$ $a l$. $(1983 a)$. These strains shared many morphological properties with strains grouped into subclusters 1-1 ( $S$. albidoflavus) and 1-3 (S. griseus). The cluster contained $S$. celluloflavus DSM 40839 belonging to the category IV, yellow series of Williams et al. (1989).

Cluster 22 (Streptoverticillium sp./S. lavendulae/ $S$. fradiae) contained 133 strains. It could be divided into five subclusters $(22-1$ to $22-5)$ at the $84.0 \%$ similarity level $\left(S_{\mathrm{SM}}\right)$.

Subcluster 22-1 (Streptoverticillium griseocarneum) contained 50 strains of the genus Streptoverticillium in addition to 'Streptomyces mediocidicus' ISP 5021. This strain was recovered as a single-member cluster in cluster group F of Williams et al. (1983a) and shared the morphological properties of the genus Streptoverticillium. Subcluster 22-1 was very homogeneous and all strains conformed to the genus description of Streptoverticillium of Locci \& Schofield (1989). The groupings of Locci et al. (1981) and, hence, the classification of Locci \& Schofield (1989) could not be confirmed in our investigation.

Subcluster 22-2 ( $S$. roseoviridis) was composed of five strains, two of them from Williams group A5 ( $S$. exfoliatus) and one each from Williams groups A6 ( $S$. violaceus) and $\mathrm{A} 38$ ( $S$. prasinosporus). The other strain in this cluster was designated as $S$. olivaceus.

Subcluster 22-3 ( $S$. lavendulae) was a heterogeneous subcluster containing 43 strains, most of them classified within the groups F61 (S. lavendulae) and F62 (S. luridus) of Williams et al. (1983a) and most of them producing smooth, red spores in RF chains. The majority of the strains were melanin-positive. In addition to single strains of Williams groups A2, A6, A10, A17 and A29, the subcluster contained four strains currently classified within category IV of Williams et al. (1989), two of them belonging to the red series (S. lavendofoliae ISP 5217 and $S$. cinnamonensis DSM 40803) and two belonging to the grey series ( $S$. globosus DSM 40815 and $S$. purpeofuscus DSM 40283). These strains shared many morphological and physiological properties with the other members of the subcluster.

Subcluster 22-4 (S. misakiensis) was composed of 17 strains sharing many physiological properties with the strains found in subcluster 22-3. Three strains of Williams group F66 (S. misakiensis) were found in this cluster, as well as strains from groups A2, A12, A14 and C44. Many strains produced a grey spore mass in RF or $\mathrm{SP}$ chains. Only a few strains were melanin-positive. The subcluster contained $S$. cuspidosporus DSM 41424 from category IV, grey series (Williams et al., 1989).

Subcluster 22-5 ( $S$. fradiae) was homogeneous and contained 17 melanin-negative strains, most of them producing smooth, red spores in RF or SP chains. It included $S$. clavuligerus DSM 40751, category IV, grey series, and $S$. roseoflavus ISP 5536, category IV, red series of Williams et al. (1989).

The four subclusters $22-2$ to $22-5$ fused at the $83.4 \%$ $S_{\mathrm{SM}}$ similarity level.

Cluster 25 ( $S$. lydicus) consisted of 17 strains, the majority of which belonging to Williams group A29 ( $S$. lydicus), in addition to four strains designated as $S$. hygroscopicus, which are currently classified within $S$. violaceusniger (Williams et al., 1989). In most cases, the strains had SP spore chains and their spores were grey and smooth. The cluster contained $S$. kurssanovii ISP 5162 and $S$. noursei DSM 40635 from category IV, grey series of Williams et al. (1989).

Cluster 30 ( $S$. albus) was a homogeneous cluster, containing 17 strains, composed mainly of strains from Williams group A16 ( $S$. albus). The strains produced smooth, white or yellow spores in SP chains and produced no melanin, thus sharing the features of two strains from the white series of category IV ( $S$. gibsonii DSM 40959 and $S$. rangoon ISP 5452) according to Williams et al. (1989). One strain designated as $S$. antibioticus, sharing the morphological features of strains found in that cluster but different from those strains of subcluster 1-5, was also grouped within cluster 30 .

Cluster 33 (S. alboflavus) contained many strains from Williams group $\mathrm{A} 10$ (S. fulvissimus) and all three members of Williams group E54 (S. alboflavus). In addition to these, some strains of $S$. kanamyceticus were found which were grouped into cluster B42 (S. rimosus) by Williams et al. (1983a). All the strains produced smooth, white or red spores in RT chains. 
Cluster 35 (S. rimosus) consisted of the majority of the $S$. rimosus strains (Williams group B42) and additionally contained two strains ( $S$. djakartensis DSM 40743 and $S$. erumpens DSM 40941) from the grey series, $S$. peucetius DSM 40754 from the red series and $S$. mauvecolor (DSM 40827) from the violet series of category IV (Williams et al., 1989). It was a homogeneous cluster and the majority of strains produced smooth spores in SP chains, or rarely in RF or RA chains.

Cluster 69 (S. ochraceiscleroticus) comprised 10 strains, four of them belonging to cluster A40 ( $S$. phaeochromogenes) of Williams et al. (1983a). Several strains of the former genus Chainia were also grouped within that cluster. Strains produced smooth spores in SP chains. Two strains from the grey series of Williams et al. (1989) were found within cluster 69 and also shared the morphological features of the cluster, among them the type strain of $S$. violaceoruber ISP 5049 ${ }^{\mathrm{T}}$, which was not recovered with the majority of related strains in cluster 6 (S. rochei).

Cluster 76 (Streptomyces sp./Nocardia sp.) contained strains assigned to different genera (Table 1).

\section{Composition and characteristics of the minor clusters and single-member clusters}

Cluster 3 ( $S$. gelaticus) contained three strains, two of them from cluster A1C and a single-member cluster of Williams et al. (1983a). They produced smooth, grey spores in RA or RF chains.

Cluster 5 (S. parvus) contained five strains, all from different clusters of Williams et al. (1983a). They produced smooth, yellow spores in RF or SP chains. No strain was able to produce melanin.

Cluster 8 (Streptomyces sp.) contained four strains, of which two were designated as $S$. scabies. None of these strains were used in the studies of Williams et al. (1983a) or Lambert \& Loria $(1989 a, b)$.

Cluster 10 ( $S$. sparsogenes) was composed of two strains, which produced grey spores in SP chains but were physiologically different from other $S$. violaceusniger and S. hygroscopicus strains found in clusters 51 and 53 to 57 of the present UPGMA $/ S_{\mathrm{SM}}$ analysis.

Cluster 11 ( $S$. psammoticus) contained five strains which had grey, blue or green spores in SP or RF chains. The strains shared many properties with those found in major cluster 9. These two clusters were joined at the $80.5 \% S_{\mathrm{SM}}$ level.

Cluster 12 (S. aurantiacus) comprised two strains producing smooth, red coloured spores in RT chains, which were similar to the strains in cluster $13(S$. niveoruber). These two clusters fused at the $80.6 \%$ similarity level $\left(S_{\mathrm{SM}}\right)$.
Cluster 14 (Streptomyces sp.) contained two strains, designated as $S$. albus and $S$. scabies, respectively.

Clusters 15 ( $S$. massasporeus), producing violet, smooth spores, 16 ( $S$. lanatus), producing blue, spiny spores, 18 (S. griseoruber) and 19 (S. cyaneogriseus), both producing grey, smooth spores, were single-member clusters. Two of them (clusters 15 and 19) were also recovered as single-member clusters by Williams et al. $(1983 a)$. All these strains had SP spore chains.

Cluster 17 ( $S$. griseoloalbus) consisted of two strains with yellow, smooth spores in RF chains; of these, strain $S$. griseoloalbus ISP 5468 is grouped into species category IV, yellow series of Williams et al. (1989).

In cluster 21 ( $S$. thermovulgaris), three thermophilic strains were recovered (Williams group A36), in addition to two mesophilic strains producing grey, smooth spores in SP chains.

Cluster 23 ( $S$. griseolus) contained two strains, producing RA chains of smooth red or red-greyish spores and physiologically very similar to subcluster $22-5$. This cluster contained $S v$. avidinii ISP 5526, which is currently classified as species incertae sedis in the genus Streptoverticillium (Locci \& Schofield, 1989), although it has been described as a typical streptomycete (Shirling \& Gottlieb, 1972).

Cluster 24 (S. fungicidicus) was composed of four strains, producing grey, rugose or smooth spores in SP chains and showing much similarity to the strains found in major cluster 25 ( $S$. lydicus). These two clusters were joined at the $81 \cdot 3 \% S_{\mathrm{SM}}$ level.

Cluster 26 ( $S$. flavidovirens) contained two strains producing smooth, yellow or greyish spores in RF or RA chains.

Cluster 27 ( $S$. fradiae) contained a further two strains of $S$. fradiae showing the morphological properties of strains grouped into major subcluster 22-5, but physiologically different from strains grouped into subcluster 22-5 (see Tables 2 and 3).

Cluster 28 ( $S$. prasinus) contained two strains not considered in the study of Williams et al. (1983a). The strains were morphologically different. $S$. prasinus DSM 40774 formed green spores in RA chains, while $S$. achromogenes subsp. rubradiris formed white spores in RF chains.

Cluster 29 (S. loidensis) was a single-member cluster and was not considered in the study of Williams et al. $(1983 a)$.

Strains of cluster 31 (S. cacaoi) and single-member cluster 32 (S. albus), which contained the type strain of $S$. albus, ISP $5313^{\mathrm{T}}$, joined the major cluster $30(\mathrm{~S}$. albus) at similarity levels of $81.4 \%$ and $80.0 \%\left(S_{\mathrm{SM}}\right)$, respectively. They shared all of the morphological and many of the physiological properties of strains found in major cluster 30. 
Single-member cluster 34 (S. fulvissimus) contained the type strain and joined major cluster 33 (S. alboflavus) at a similarity level of $80.4 \%\left(S_{\mathrm{SM}}\right)$.

Two strains of $S$. rimosus were grouped into cluster 36 ( $S$. rimosus). They shared the morphological properties of strains grouped within major cluster 35 (S. rimosus) and joined this cluster at a similarity level of $81.0 \% S_{\mathrm{SM}}$.

Clusters 37 (S. varsoviensis), producing yellow, smooth spores in RF chains, 38 (Actinomadura luteofluorescens), producing yellow spores in RA chains, 39 ( $S$. caelicus) and 40 ( $S$. spheroides), both producing yellow, smooth spores, were recovered as single-member clusters. Two of them (clusters 38 and 39) were not included in the study of Williams et al. (1983a).

Cluster 41 ( $S$. endus) was composed of two strains, both producing rugose, grey spores in SP chains. These strains were physiologically different from those found in clusters 10,51, 53 and 54, which all contained strains currently grouped into $S$. violaceusniger (Williams et al., 1989).

Single-member clusters 42 (S. olivaceus ISP 5072) and 43 (S. niveus ISP 5088) contained strains grouped within Williams groups A1C and A1B, respectively. These strains shared the morphological properties of cluster 1 , but were physiologically different from strains grouped into subclusters 1-4 and 1-2.

Clusters 44 and 65 (Streptomyces sp.) were each composed of two strains, not assigned to named species, and single-member cluster 45 contained a strain designated as $S$. scabies which differed from those found in clusters 6,8 , and 9 . Single-member cluster 46 (S. pallidus) contained a strain grouped into cluster A18 (S. cyaneus) of Williams et al. (1983a), but not listed among the synonyms of that cluster (Williams et al., 1989). Singlemember cluster 47 ( $S$. anulatus) produced yellow, smooth spores in RF chains and 48 ( $S$. parvulus) produced grey spores in SP chains.

Cluster 49 ( $S$. roseiscleroticus) contained two strains producing greyish, smooth spores in SP chains, one of them $(S$. ruber) currently classified within the red series of category IV (Williams et al., 1989). Both strains belonged to the former genus Chainia which has been reclassified into the genus Streptomyces (Goodfellow et al., 1986).

Cluster 50 ( $S$. violatus) was also composed of two strains with grey or white spores in SP spore chains.

Clusters 51 to 57 (S. violaceusniger, S. hygroscopicus) were formed from the majority of strains designated as $S$. hygroscopicus and $S$. violaceusniger (Table 1). The strains produced grey, rugose spores in SP chains and produced no melanin. One strain of the grey series of category IV (S. antimycoticus ISP 5284) was also found in cluster 51, sharing the morphological properties of the majority of strains. Single-member cluster 58 (S. caeruleus ISP 5103) classified within the grey series of category IV, was morphologically and physiologically very similar to strains found in clusters 51 to 57 .

Single-member cluster 52 (S. bluensis) produced smooth, blue spores, clusters 60 (S. malachiticus) and 62 (S. xantholiticus) formed smooth, grey spores, and cluster 61 ( $S$. bellus) produced blue spores, all in SP chains

Single-member cluster 63 ( $S$. albosporeus) of the red series of category IV of Williams et al. (1989) also produced smooth spores in SP chains.

Cluster 64 (' $S$. longissimus') contained three strains not included in the study of Williams et al. (1983a). The cluster contained strains possibly not belonging to the genus Streptomyces, with ' $S$. mediterranei' now classified into the genus Nocardia as $N$. mediterranei, and ' $S$. longissimus' listed as species incertae sedis in Bergey's Manual of Determinative Bacteriology, 8th edn (Pridham \& Tresner, 1974b).

Cluster 66 ( $S$. flocculus) was composed of strains morphologically similar to those grouped into clusters 30 , 31 and 32, but physiologically different (see Table 3).

Single-member cluster 67 (Nocardia sp.) contained a strain morphologically and chemotaxonomically different from Streptomyces. It could be assigned to the genus Nocardia on the basis of chemotaxonomic analyșes (unpublished results).

Cluster 68 (S. sulphureus) was composed of two strains producing smooth, yellow spores in SP chains.

Single-member cluster 70 contained a strain of $S$. fradiae physiologically different from those found in subcluster 22-5 and cluster 27 (Tables 2 and 3). Strain $S$. poonensis ISP 5596 formed single-member cluster 71 . This strain shared many properties with those found in cluster 49 (S. roseiscleroticus). Single-member cluster 72 contained a strain not assigned to a species and singlemember cluster 73 comprised a strain of $S$. olivaceus which was physiologically different from those found in subclusters 1-2 and 1-4 (Table 2). Single-member cluster 74 was comprised of $S$. erythrogriseus, which is currently classified in the red series of category IV (Williams et al., 1989). Single-member cluster 75 (S. bambergiensis) contained a strain producing green spores with a hairy surface in RA chains, also recovered as a single-member cluster in the study of Williams et al. (1983a).

Single-member cluster 77 (S. ipomoeae) comprised a strain currently classified within the blue series of category IV (Williams et al., 1989). Clusters 78 to 87 contained small numbers of strains or were singlemember clusters, in several cases named after recognized species or clusters, but different in their physiological properties (Tables 2, 3 and 4) and thus forming separate phena (see Table 1). 
-ds saokmoldalis $\infty \mathrm{N}$ -ds saskmo1daגis $\varnothing^{m}$ !nupud!l $S$ 의

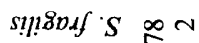
snaגnydjns $S: 0 \mathrm{~N}$ snpn.xoy $S$ 요 -ds saskuoldalts $\approx \mathrm{N}$ snumss!isuol $S$ J $m$ sno?dossod8Ky $S$ in $\mathrm{N}$ snotdossos8ky $S$ in $\mathrm{N}$ da8!usnaวplo!a $S$ t $n$ גa\&!usnasplo!a $S$ กิ

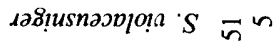
sniploia $S$ ह snollodapsipsod $S$ q $\mathrm{N}$ ds sarkmoldans snpua's $\mathrm{F}$ N snsoumd 'S e $\mathrm{N}$ lovops $S \mathrm{~m}^{\mathrm{m}}$ snutsped ' $S$ 少 ap!ppuf $S \widehat{\sim}$ suдmaop!apy'S 유 $\mathrm{N}$ snग!p!ग!?unf $S \underset{\mathrm{N}}{\sharp}$ snloas?18's $\overbrace{}^{2}$ s!p!nlaossod $s$ ஸ̃n

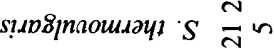
snqpopozsild $S=N$ -ds sajkimotdans $\pm N$ snop!lupגno $S \simeq N$ snolloumuns $S=$ 的 sauว8osıdds $S \subseteq N$ 'ds saskuoldans $\infty \forall$ snaud $S$ \& $n$ snj!lppas $S \mathrm{~mm}$

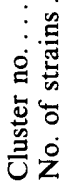

$0000000-0000000000-0-0000000000000000001$

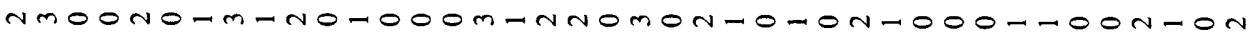
mmo-0000NONNOOMNNNNNO-0000m00000000-000

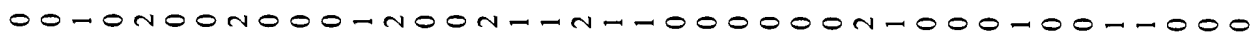

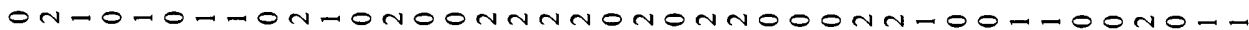
O-TOmO-mom-OmommNmmoNOm-NO-mmm-ONO-OM-NN

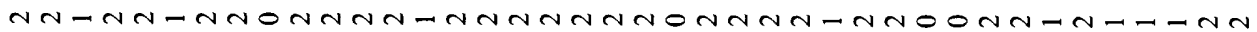
mm-mmoNmNm-mm-OmmmmmmoNmNm-mmoOON-mOm-O-

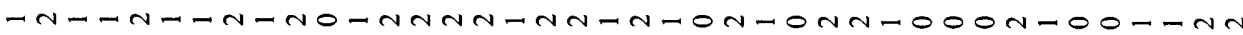

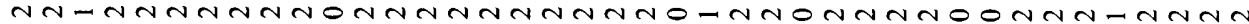

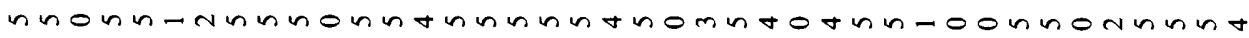
mmN-mOMmNmOmmmmmmmmmmONmOONmm-OONmN-mm-N $\forall \forall-m n-n n \nabla n-n n m \forall n n n n n n O m n n-m n m-0-n n \nabla 0 \forall \forall n n$

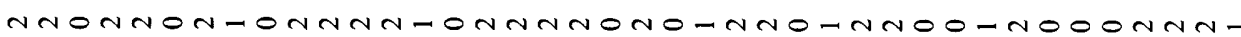

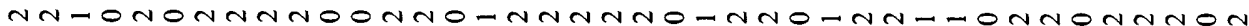

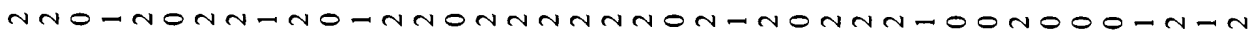

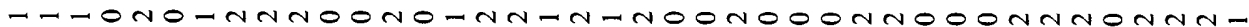

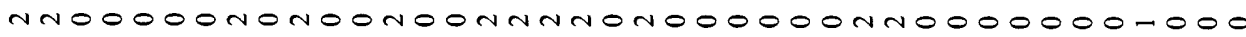
mmoNmommomommoOmmmmmmoNmmoOmmoOONNM-MONN -nnononnonoonoonnnnnnoonnomnn-obnonobonn

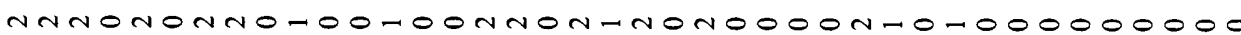

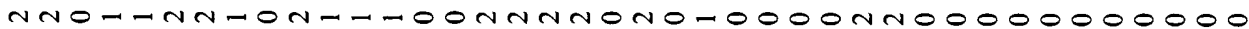

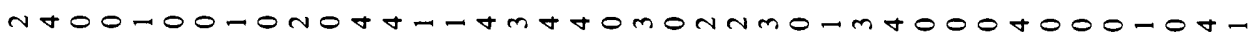

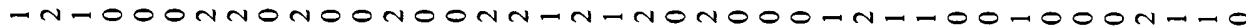

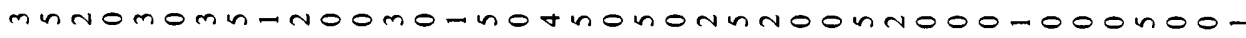

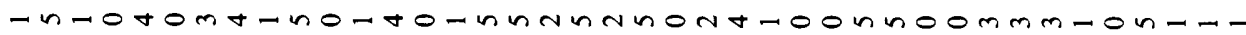

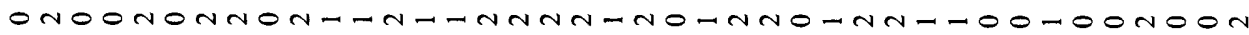
onoononnon-n-00n-0nononnhomn-noonodon-nn

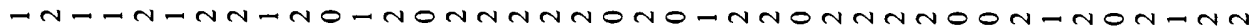

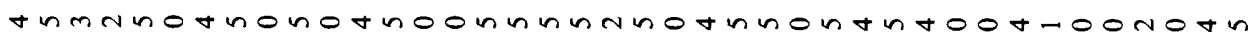

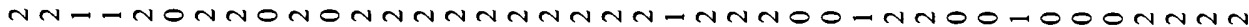
ntO-

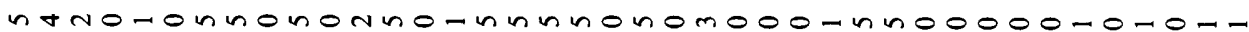
mmNm-Omm-NOmmoOmmmm-moNn-OOMmOOOMm- NO-M

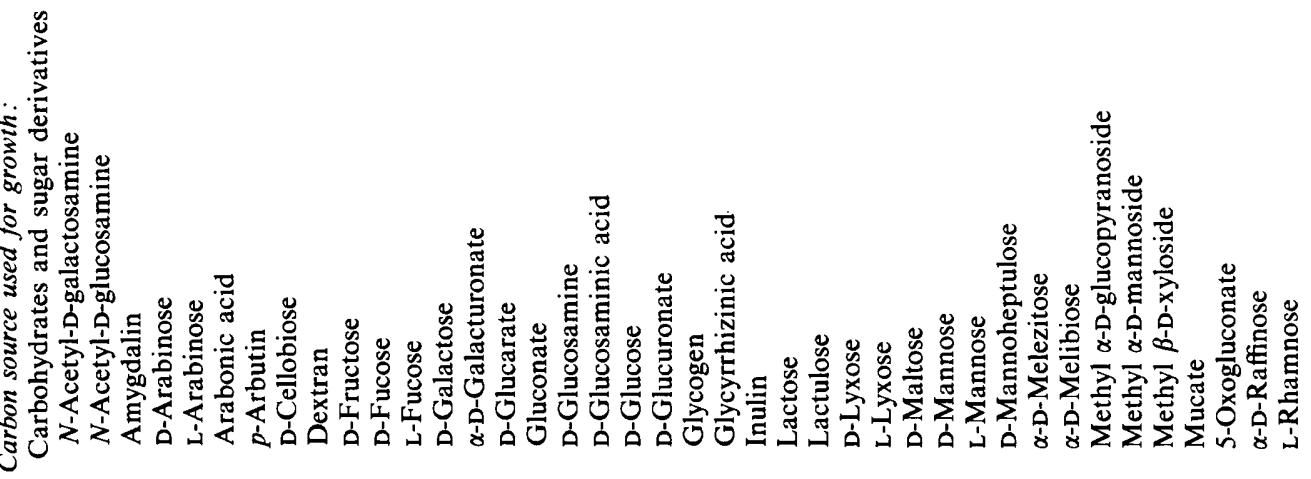


$000000-000$ ond moloog - $000 \mathrm{mo0} 000$ o-m nom- $-4-0 n O n-n o$ mmmomommmo nNMONONNNO mmmomomnmo nNMONOODTO nononon-no nNmomotono momomom-no nmmonommino nN-ONON-nO nNMTNOnNho nONONONONO n-nONONNNO NOOONONOOO m-momonNmo nONONONOHO onnonom-ho nhnonon-oo m-OOmOtono n-nonon-mo - omonolono $m+t 0 \ln 0 \operatorname{mog}$ no-OnONONO nnNonomono - onononne ntmonomnno nnnononono thnotonoto nomono no no mamomomnmo
$0-000000-00$

- $-000 \mathrm{~m}-0-00$ $00000 m-0000$ $00000-00000$ $-\mathrm{n}-\mathrm{O}-\mathrm{n}-\mathrm{nO}-$ onoo omo-moo nNmo onNNMOO -moo ommnmonNoO OnN-nOO -nNo nNNNNON ma-0 nmtnino mn-O mm-nmON $-n-0 \quad \operatorname{mn} \tan 0-$ nNoO OnNThOON-I ON-NNON modonnonoo moon-n-nNo n-OO nNoONON moommntmoo onoo ondonoo OODO ONOOOOO nodo onnodoo nhooghtom$-7-0-4-m 00$ $0-000 n-0-00$ $0 n-0$ on $\tan 0 \mathrm{~N}$ onomon-nnoo 000 onnoloo ondo onn-noo -n-o onm-moo nNoo onnonoo omoootmomoo -noo on- nno n-OO 0 mom- 0
00000- $-0-00-0$ maOONNNo0000$n+N-n N N$ $m m m n N m$ nNoph-n $m m o N m m m$ No00000 nNommooo-on $m \mathrm{~N}-\mathrm{OmNm}$ ngodn N-IONOn- $0000 N$ NNOM-N $n-0 n-n$ NNTONNN $m m m-N m m$ NoO-OON $\mathrm{N}-O \mathrm{NN}$ $\mathrm{n}-\mathrm{n}-\mathrm{n}$ NoO000nom$n n+\forall+n n$ mnnNNm no- $-0-n$ $\mathrm{n}-\mathrm{n} n \mathrm{n}$ $-100-\mathrm{N}$

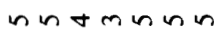
nNM-NM m-ooonA-n- -0 mNNMNm
$000000-0000000000-00-0-0-$ $00-N 00 m-0000-0000000000 N-$

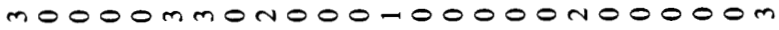
$000000000000 N 00000000000-$ $0-0-00 n 0-700 n-700 n 00 \mathrm{nnnn}$ mO-mOMmNmOONNNOOHNONOMmmm

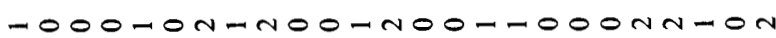
$m--m-m m-m-n m 00 N-N m 0 m m m-m$

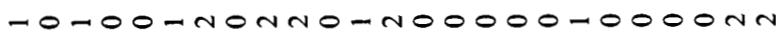

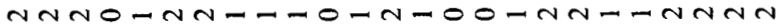

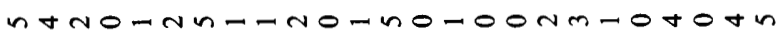
mo000mm-ImONmOOOOONNONTmm

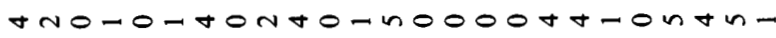
m-nn-modnomolomomolnono

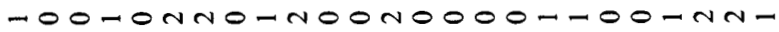

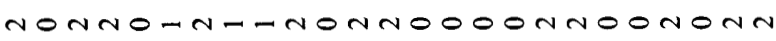
nodoonnoonoonodoononomono

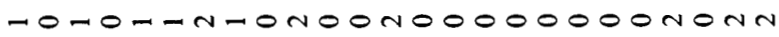
NOOOONMOOMOOMOOOONMONmmm-

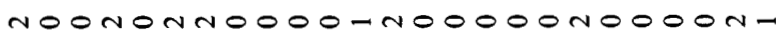

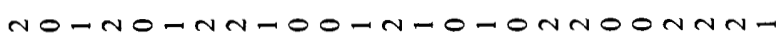

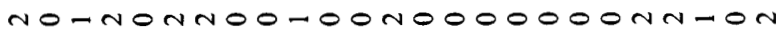

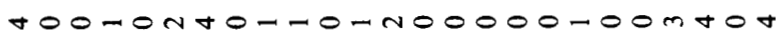

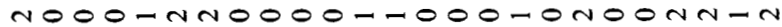
n-ONOnn-000-700000m0-00nm

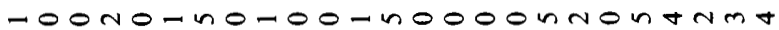

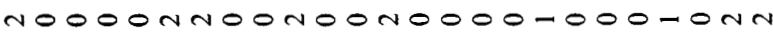
noo-onnoo-0-000000-0000nn

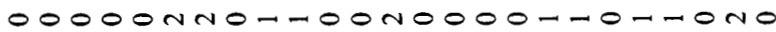
noo-0mnot-10-no000mmotnnmm

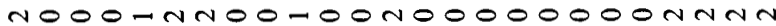

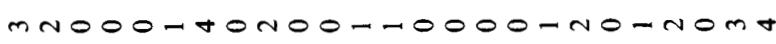
o- omoonoo-00no000-000-0nmoONOmmoOmo-m-OOOOOMONmmmm

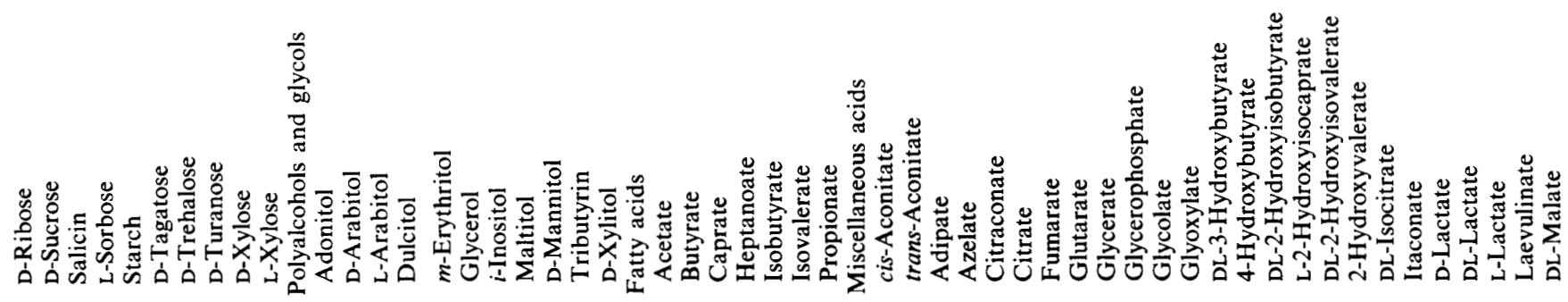


$-00000000-0-00000$ m-oodnoomnonmolno

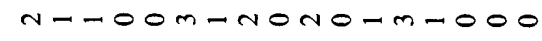
$-0000-000 n-0-0000$ no-0.nnN-nN-400000 m-100mm-0mn-mo0o-
$-000-$ $0000 \mathrm{n}$ $0000-$ $00-00$ $-0-0 N$ $0000 \mathrm{~m}$ $-000-$ $0000 \mathrm{n}$ 00000 $-n-n-$ 00000 00000 $0000 \%$ 00000 0000N O000N $0000-$ $0000-$ $0000 \mathrm{~m}$ O000N OOOON 00000 $0000-$ $0000-$ $0000 \%$ oodon $0000-$ OOOON $0000-$ $0000 \mathrm{n}$ 0000. 00000 $0000-$ 00001
$000000-0-100000-000-10-000$

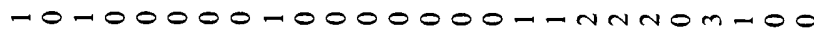
o-mnoomnodoonoomon-nomolo $00-1000 n-0000000 n 0 m 000 n 000$

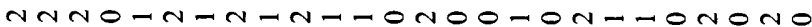
NTONOMNmmm-momoO-OMNOOMO-O

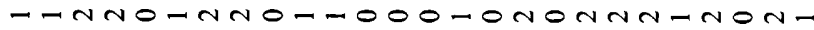
mNmmo-mmN-DO-moomommmNmomm-m-00-n-0000-0000n-00no0

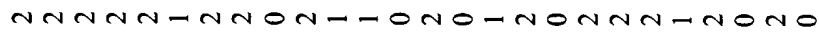
-ntt-0nno0000nomtonnmnno-o mnOmomm-n-m-moOmonnNOmONO

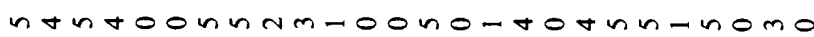
non-00nnn-000noononnonnono -nnnobnnob-00no000n-n-nono

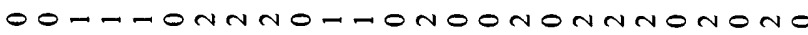

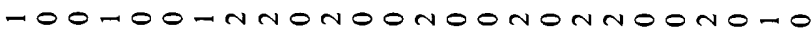
onnhoomnn-000nobnomnnono-o Nmmn-OOmmo-00N0000Nn-mo00 mo-0000nnoboonomnonnnonomo

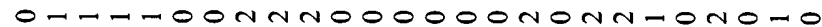
n-nho0n-n-0, no - m-

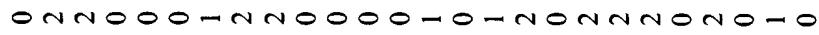
$n \rightarrow \forall$ NOnnm - m- - 000nn-0-0no-nom-n-nono onno-0-nno

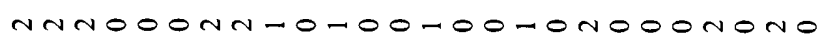

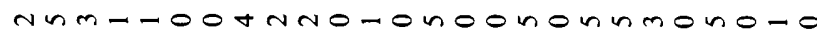

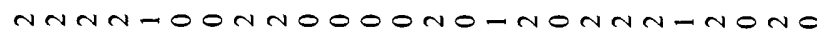

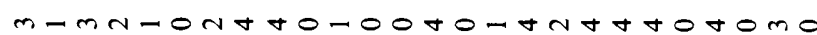

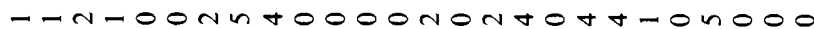
O-MN-OMnmoO-OmoOmommmomooo 
$000000-00-00000000000000000-000000-000$ $0000 m m 000-00-000-0000000000 m 00 N 000000 N$ $000-m n-00 N 00-00000000000000 N O-m 00-m 0-0$ $0000 n-000 N 00000000000000000-00 N 00$ - 0 OOOONNOOONO-ONNONOOOOO-ONNONOONOONNOON

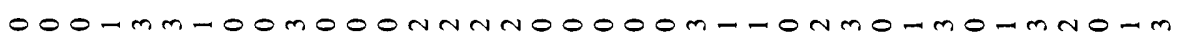

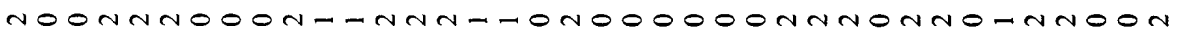
N-Tmmmmo-moOmmmmmomooONN-mmmo-mN-mmmmm $000-N 000-000000000000000-0-00-0000-1-$

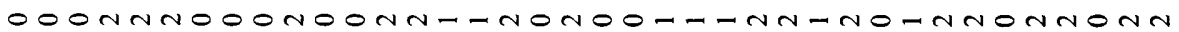

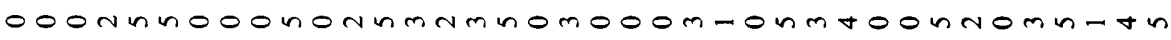
O-ONmm-OOMOO-N-O-O-OOO-OONNmOOMNOMm--m ooo-nnm-ontontmomotooo-0onononntonnonn

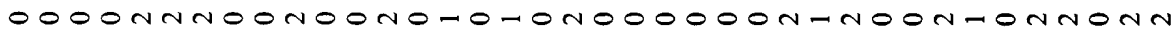

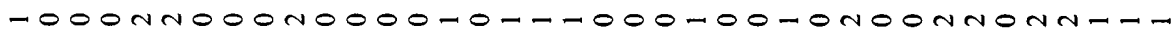

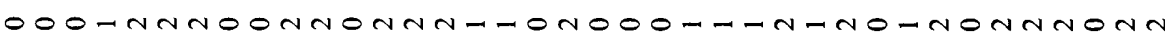

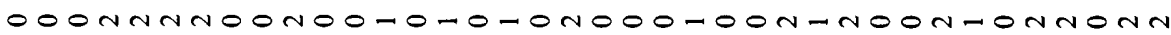

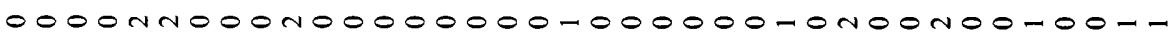
OOONMmNOONOOH-WO-ONOOOOOOO-MOOMOOMmOON

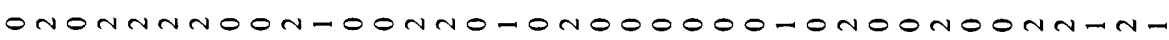

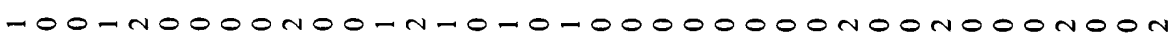

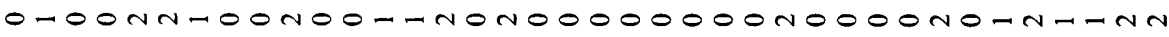

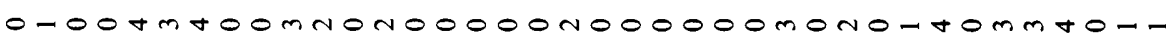

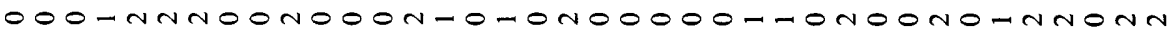

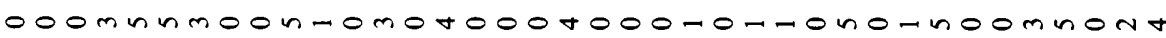

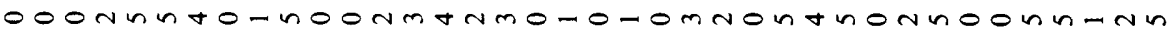

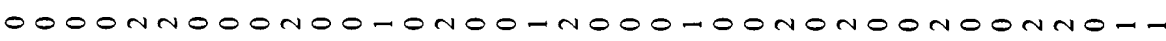

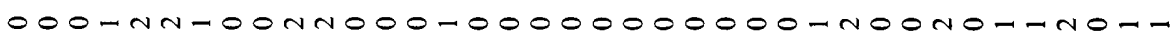

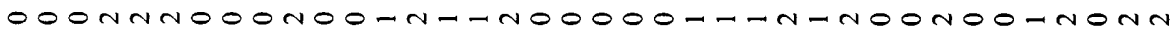
000ninttoono onmmonomo0000-n-moonontmonn

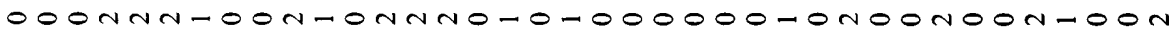

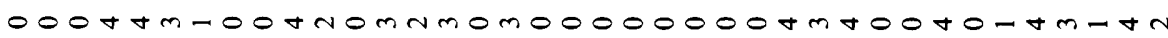

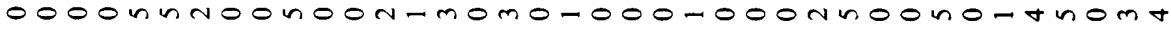

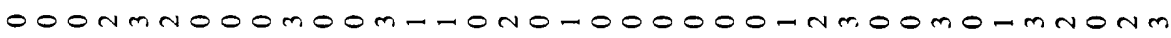

0000000000000000 oodoono-onnom-on $00000 m 000 m n-0000$ - -00000-0n-00000 OO-OONOO-OOO-000 $000000000-10000-$ $00000-000-100-0-$ oodoonoo-00-0000 $0000000000000-00$ -OOnONO-ONNOONOoo-0omooominootoo $00000 \mathrm{~m}-00 \mathrm{mmo}$ mo - $0000 \mathrm{nomot}$ toomoo o-OOONOOONNOOOOO $00000 N 0000000-00$ O-OOONONONNOOOOO OOOOONOOONNOONOO

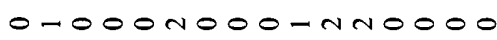
$00000 m-00 m m-0-00$ o-OOONOOONNOOOON ONOOONOOONNTOOOO $00000 N 000-000000$ $00000+000-t 00000$ $00000-000$ NNOTO0 otoootooonnmolom omooonoognn-000 in 00000-000NNO0000 o-OOONOOONNOOOO$00000-100 n-0000-$ omooonooonno0000 OOOOONOOOH-OO-OO ONOOO-OTOMnOOMOM $00000700-n n m 0000$ 00000m000nm-0000

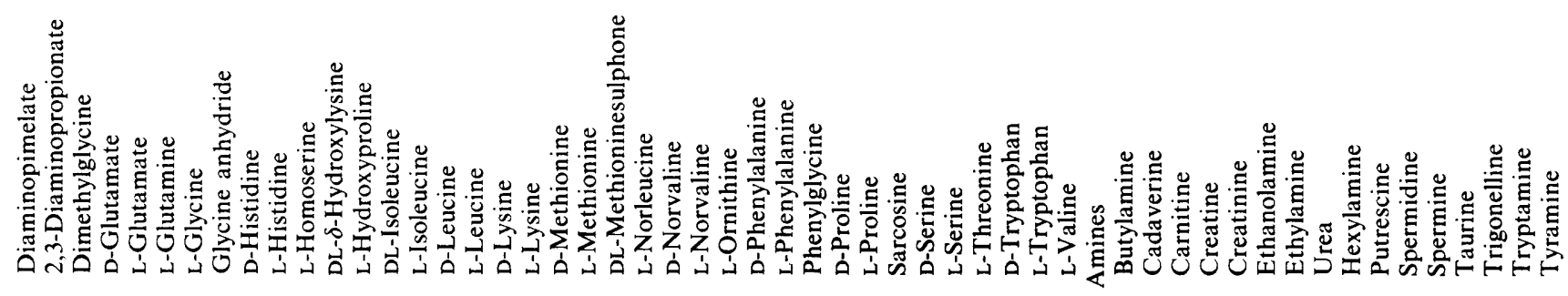


0000000000000 0000000000000 $000-00-00000 N$ $0000000-00000$

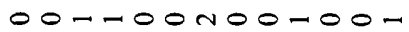
000-mo-00000m $000000-00000 \mathrm{~N}$ -O-nNOMOOJOON $0-00000000000$ om-nOOnOO-OON $0-0-00+000000$ $000000-000-0-$ $000 m-0-00000 m$ ONOO-00000000 $000000 n-00000$ -n-noo-o, nom $0000-00000000$ 0000000000000 OMOMONOOOOOOO onon-OnOOJOON OOODOONOO000$000-00000000-$ omo-00000000$0-0000000000 \mathrm{~N}$ $000000-00000-$ $000-00000000 \mathrm{n}$ 0000000000000 $000000 N 000000$ $000000 N 00000$ oormoomooo-on $000000-000000$ $0-1000+00000-$ $0-0 m-0000000 m$ $000-00-000000$
$00000000000000-000000000$ $000000 m 00-0000-00-0-0000$ $000-0000000000-N 0 m 000000$ $00000000000000-10 N 000000$ $00000000000000-n O-000000$ $000000-00 m 0000 m n O-O N-000$ $-00000000-0000 N N O N O-0000$ - $00000 m 00-0000 m m-m 0 m-000$ $000000 N 00000000000000000$

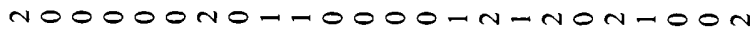
nooon-mo0000-00n-mothoonOOOOH-O00000000-mo-nOOO mo00mo-0000000-moton-000 $000000-00-0000-10 N 0-000-$ $00000000000000 N N O N 000000$ - - O-O-NO0000000N-NO00000 $-0000000000000 N N O-000000$ $000000-0000000 N N O N 000000$ $000000000000000 m 0 m 000000$ 000000-0000000NNONO-0000 $000000 N 0000000-10 N 000000$ $000000-0-100000000000000$ $-0000000000000-00 m 000000$ $000000000-0000 N N O-000000$ $000000-0-10000+00-000000$ $0000-000-00000 \operatorname{tann} 000000$ $000000-0000000-1-00 n 0000$ $-00000000-0000-n+n 000000$

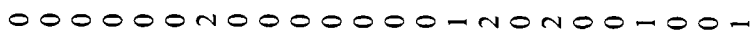
$000000-0000000 m+0 m 0-0-00$ $00000000000000-00-000000$

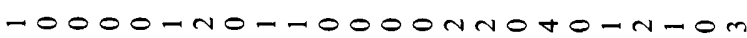
$00000000-10000+n 0 m 0-H 000$ $-0000-00000000 m-0 N 000000$
00000000 mNn-OM$-1-00-\cdots$ N-O000MNNNNNNNN mmmmm- nNMTOMT mmmm-NNNN-DO-OO NNN-T-OO nin m n m N N $m m m m-m m m$ $n n-00000$ $-1000-00$ NNNNNNNN $n-n n-0,00$ NND- - NNNNNTNN mmmmmNNNNNNINNO NNNOOHO nNNNOOOO $\forall+\nabla m o \forall+C$ NNTO0000 m n $A$ NOOO nungmunn NNOOONNT nNMDHnNT00000 ntonm NNN NNN-DNAmnON-O nnnt mmmmm- - o

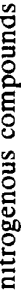

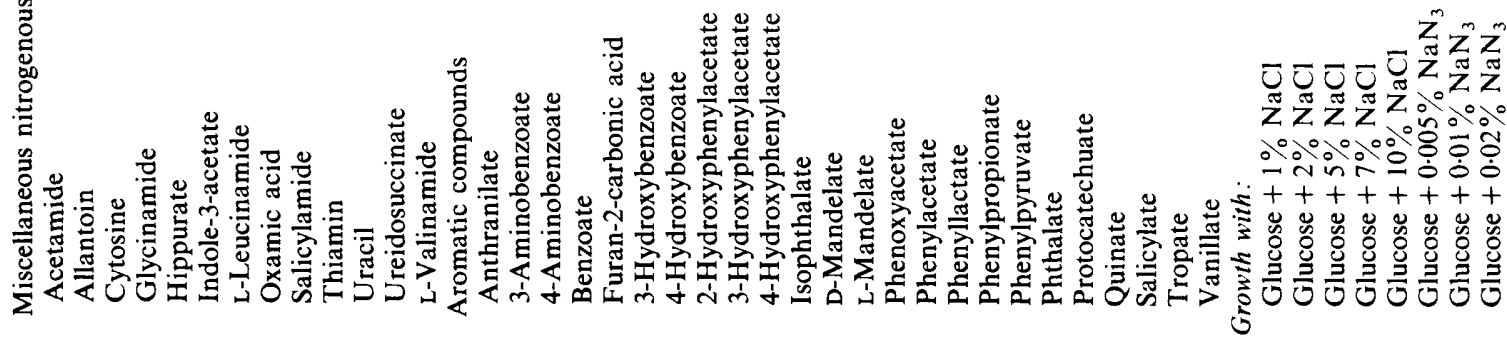




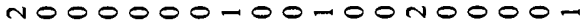
mom-m-ONOmmONTNmOOm

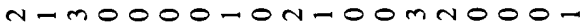
nonnhoobonnom-nNobn

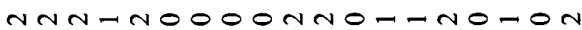
mom-moO-OmmoONmmN-m

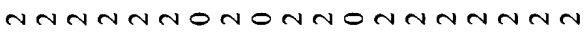
mom Nm- $-N-N m 0 N m--D-m$ nnnnnhononnonnh-7-n

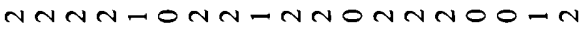
$\forall \forall n-m-0 n 0 n n 0 \operatorname{trn} t 000 n$ mNmONOONONmO-m-OOM$n \forall n m m-m \forall-n n O \forall+n N O m n$ nonn-oondnnonn-mnonnhoo-onnonnhnom $4-4-400 n$ n. non-nho-n

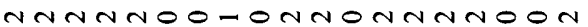

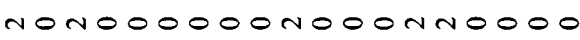
mmmmm-NmOmmNmmmNOOM $-0 n-400 n-n n-n n-n-m$ non-noomonno-onnogn

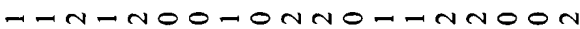
totoomomotmoonm-0onon-nO0, nom tonnmo-nonnottunoor nNmtnoOmonn-Nthnoln

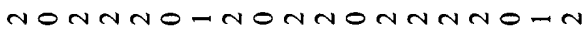

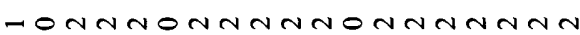
nonnhonnonnonnhn-nh nonnmm-nonnonnmm-nn

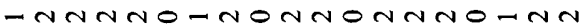

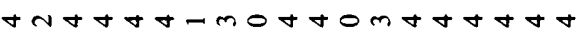

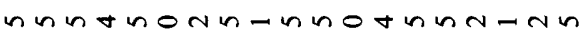
mmm-m-NmommoNmmmoNm
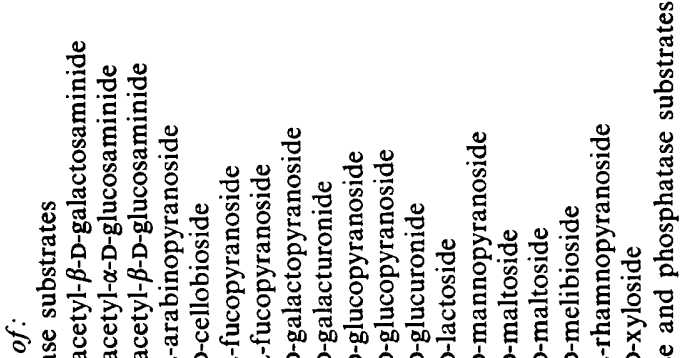

OnOn-m-O ommmmnn $0 m m m m m m$ onNNMNNN

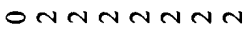
ommm NmNm $0 \mathrm{nnnnNh}$ $0 m m m m m m$ $0 n+4 n n+4$ $0 \mathrm{nNnNNN}$ Onntunnn Omm Nmmmm O $\forall n \tan n$ onNNNNNN onNNMNNh onnNnNnN nNNNNNNN onNnNNNN $0 m m m m m m N$ onNnNNN$0 n-n N 4 n+n$ onNNNNNN $0,4 t \lambda t 4 t$ onNNNNNh Onnnm nn n Onnnnn $\forall n$ onNhNNNN onNMNNNN $0 \mathrm{nnnNhN}$ onnnmunn onNNNNNN $0 \forall \forall m \forall \forall \theta$ O $n \operatorname{tgn} n \operatorname{mn}$ $0 m m m m m m$
$000-00000000 \mathrm{~N}-0$ oonnnom-hnomo OOMmmomnNmm Nmm N oonnNonNmNnON-O $0-4 n-4 n+4-04 n n$ $-m m m-0000 m m 0 m m N$ o-nnnonnnnnonoo $m m m m m-m m m m m o m m m$ oonn-0,nnn-0-00

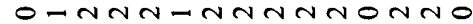
ootnno-nnmn-mno

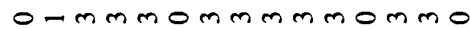

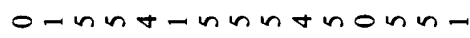

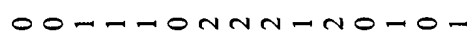
oonnnonnnnNonoo oonnnonnonnon-

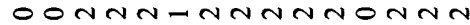
oonnnoom-nnonoo OOmmNOmmmmm-m-m

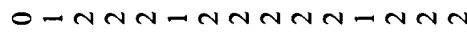
oonnhonnh-non-o $-\pi-n$ nOn-m-ThN $00+470-m m+40,00$ oonnNonNNWN-n-n O O $n n n-n \nabla+\tan \operatorname{rnm} n$ o-nunommm nnnmom oon-nonNNNNONOO

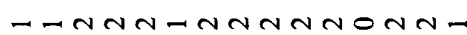
o OnO-ONnN-nONOO oonntonnminomooonnNonNNMNON-

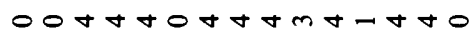
oonnnounnmmon-m o-mmmommmmmomm-
00000000 00000000 $-0000000$ $-0-00000$ 00000000 $0000000-$ 00000000 $0-000000$ 00000000 00000000 00000000 00000000 No000000 00000000 $00-0-0-1$ noOOnOH$-0-0-0-1$ $0000000-$ m-monom NOOONONT 00000000 $-000-0-0$ $-1000000$ $-0000000$ $-00000-0$ 00000000 $00000-00$ 00000000 $-0-0000 \mathrm{n}$ N-O-00$00-0-0-0$ 00000000 $+-10-0$ m n mmoool-

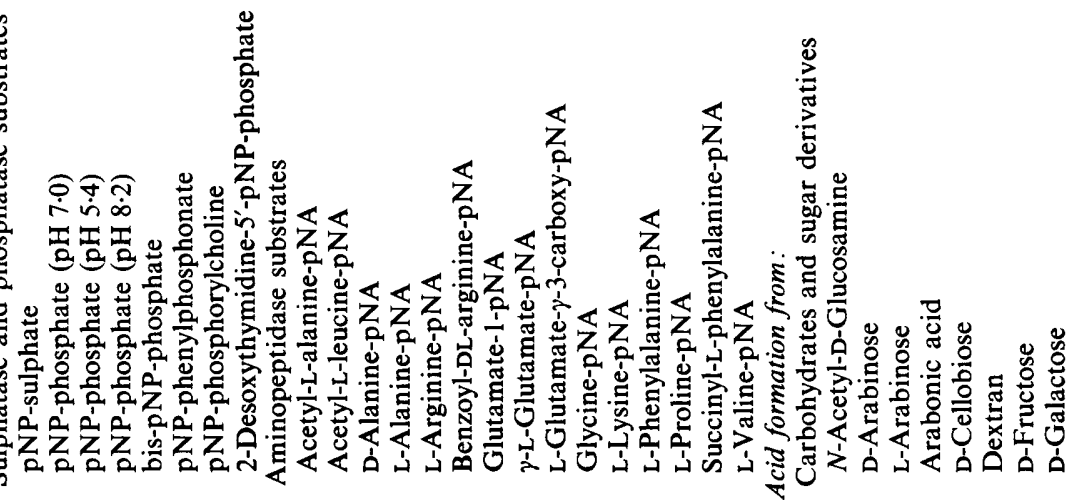




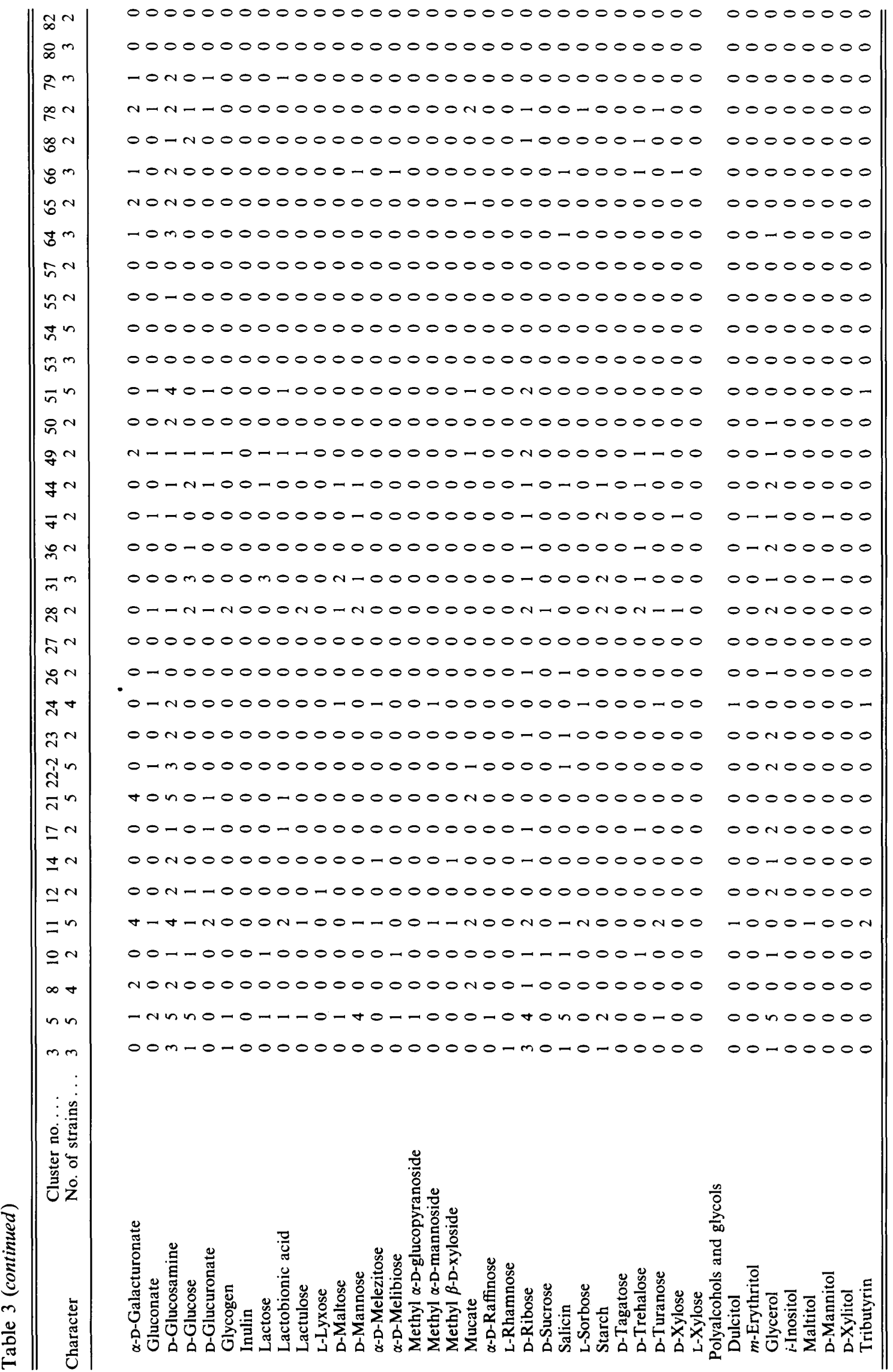




\section{Overlap of clusters}

Only statistically insignificant or no overlaps with other major clusters and subclusters were found for the $S_{\mathrm{SM}}$ subclusters 1-1 ( $S$. albidoflavus, Williams group A1A), 1-2 (S. halstedii, Williams group A 1C) and 1-3 (S. griseus, Williams group A1B), cluster 2 (S. exfoliatus, Williams groups $\mathrm{A} 5$ and $\mathrm{A} 6$ ), cluster 9 (S. violaceus, Williams groups A18 and A19), subcluster 22-5 (S. fradiae), and cluster 30 (S. albus). Cluster 33 (S. alboflavus) overlapped significantly with cluster 35 ( $S$. rimosus). Subcluster 22-1 (Sv. griseocarneum) overlapped with subcluster 22-3 ( $S$. lavendulae) with an index of overlap $V_{\mathrm{G}}>10 \%$. Subcluster 22-4 (S. misakiensis) also showed overlap with subcluster 22-3 ( $S$. lavendulae) with $V_{\mathrm{G}}$ just over $9 \%$.

Most of the overlaps were found between major clusters and adjacent minor clusters, with $V_{\mathrm{G}}$ values ranging between $9 \%$ and $22 \%$. The major cluster $9(S$. violaceus) showed overlap with clusters 11 (S. psammoticus), 12 (S. aurantiacus) and 13 (S. niveroruber), with $V_{\mathrm{G}}$ values of $9 \%, 16 \%$ and $18 \%$, respectively. All minor clusters $51,53,54,55$ and 57 , containing strains of $S$. violaceusniger and $S$. hygroscopicus, showed overlaps with one another. Cluster 30 (S. albus) overlapped with cluster 31 (S. cacaoi), with a $V_{\mathrm{G}}$ exceeding $25 \%$. Similarly, there was an extensive overlap between cluster 35 ( $S$. rimosus) and cluster 36, which also contained two strains of $S$. rimosus.

\section{Clustering of strains using the UPGMA algorithm with the $S_{\mathrm{J}}$ coefficient}

The study using $S_{\text {J }}$ coefficient considered only positive matches and therefore revealed a changed phenogram with reduced similarity levels at which the phena were recognized. The cophenetic correlation coefficient $r_{\mathrm{cs}}$ as a measure of correlation between corresponding pairs from the similarity matrix and the cophenetic matrix was 0.6929 .

Taking a similarity level of $59.6 \%\left(S_{\mathrm{J}}\right)$, a total of 78 clusters was defined.

$S_{\mathrm{SM}}$ cluster 1 was further subdivided into 35 subclusters at a similarity level of $64.6 \%\left(S_{\mathrm{J}}\right)$. The assignment to different clusters and differences from the UPGMA $/ S_{\mathrm{SM}}$ analysis are given in Table 1 and the rearrangements are described in comparison with the $S_{\mathrm{SM}}$ clusters in detail below. All of the major and most of the minor clusters were recognized, with some changes in the cluster composition.

$S_{\mathrm{SM}}$ subclusters 1-1 (S. albidoflavus) and 1-2 (S. halstedii) were each recovered in their entirety in the UPGMA $/ S_{\mathrm{J}}$ analysis, as $S_{\mathrm{J}}$ subclusters $1-1$ and $S_{\mathrm{J}}$ cluster 15 , respectively. The 81 strains grouped within $S_{\mathrm{SM}}$ subcluster 1-3 ( $S$. griseus) fell into $S_{\mathrm{J}}$ subclusters 1-2 (70 strains) and 1-20 (three strains), and $S_{\mathrm{J}}$ clusters 4 (one strain), 8 (two strains), 10 (three strains), 30 (one strain) and 47 (one strain). The corresponding $S_{\mathrm{J}}$ cluster 1-2 contained the two further strains $S$. rubiginosohelvolus ISP 5176 and $S$. baarnensis ISP 5232, both found in $S_{\mathrm{SM}}$ cluster $6\left(S\right.$. rochei). All six strains of $S_{\mathrm{SM}}$ subcluster 1-4 ( $S$. olivaceus) were found in the corresponding $S_{\mathrm{J}}$ subcluster (1-4), in addition to strain $S$. viridogenes ISP 5454 , which was grouped into $S_{\mathrm{SM}}$ cluster $6(\mathrm{~S}$. rochei). Strains found in subcluster 1-5 (S. antibioticus) of the UPGMA $/ S_{\mathrm{SM}}$ analysis formed $S_{\mathrm{J}}$ cluster 11 (four strains) and were further found in $S_{\mathrm{J}}$ subclusters 1-16 (three strains) and $S_{\mathrm{J}}$ clusters 9 (11 strains) and 59 (S. recifensis ISP 5115), which became a single-member cluster. $S_{\mathrm{SM}}$ subcluster 1-6 ( $S$. flaveolus) was split into $S_{\mathrm{J}}$ subclusters 1-10, 1-12 (single-member cluster ' $S$. umbrosus' ISP 5242), 1-13 (single-member cluster $S$. flaveolus ISP $5061^{\mathrm{T}}$ ), 1-14, 1-15, 1-16 and 1-31 (single-member cluster $S$. alboniger ISP 5043). Strains of $S_{\mathrm{SM}}$ subcluster 1-7 ( $S$. diastatochromogenes) were found in $S_{\mathrm{J}}$ subclusters 1-14, 1-15, 1-19 and 1-21. Thus all strains of $S_{\mathrm{J}}$ subcluster 1-14 and the majority of strains from $S_{\mathrm{J}}$ subcluster 1-15 were grouped into $S_{\mathrm{SM}}$ subclusters 1-6 and 1-7. $S_{\mathrm{SM}}$ singlemember cluster 1-8 ( $\mathrm{Sv}$. luteoreticuli ISP 5509) remained as single-member cluster $1-17$ in the UPGMA $/ S_{\mathrm{J}}$ analysis.

All strains of $S_{\mathrm{SM}}$ cluster 2 (S. exfoliatus) were grouped into $S_{\mathrm{J}}$ subcluster 1-7 with the exception of $S$. cinereoruber ISP 5012, which formed $S_{\mathrm{J}}$ single-member cluster 38 . The $S_{\mathrm{J}}$ subcluster 1-7 contained a further two strains grouped into $S_{\mathrm{SM}}$ subcluster 22-4 ( $\mathrm{S}$. misakiensis). $S_{\mathrm{SM}}$ cluster 3 ( $S$. gelaticus) remained intact as $S_{\mathrm{J}}$ subcluster 1-3. Four strains of $S_{\mathrm{SM}}$ cluster 4 (S. graminofaciens) were found in $S_{\mathrm{J}}$ subcluster 1-23. This subcluster contained five strains; the fifth strain belonged to $S_{\mathrm{SM}}$ cluster 25 . The two remaining strains of $S_{\mathrm{SM}}$ cluster 4 formed $S_{\mathrm{J}}$ cluster 6. $S_{\mathrm{J}}$ cluster 10 was composed of 11 strains, one from $S_{\mathrm{SM}}$ cluster 5 (S. parvus) and two from $S_{\mathrm{SM}}$ subcluster 1-3 (S. griseus); the remaining strains were found in $S_{\mathrm{SM}}$ cluster $6 . S_{\mathrm{SM}}$ cluster 6 ( $S$. rochei) was split into $S_{\mathrm{J}}$ subclusters 1-18 (61 strains), 1-10 (seven strains), $S_{\mathrm{J}}$ single-member clusters 1-11 (S. nodosus ISP 5109), 1-4 and $1-2$, and $S_{\mathrm{J}}$ cluster 10 (six strains). The remaining strain from $S_{\mathrm{J}}$ subcluster 1-18 was grouped into $S_{\mathrm{SM}}$ cluster 13 ( $S$. niveoruber); the remaining strain from $S_{\mathrm{J}}$ subcluster 1-10 fell into $S_{\mathrm{SM}}$ subcluster 1-6 ( $S$. flaveolus).

$S_{\mathrm{SM}}$ cluster 7 ( $S$. prasinopilosus) was recovered as cluster 3 in the UPGMA/ $S_{\text {J }}$ analysis. Cluster 8 (Streptomyces sp.) contained one strain which fell into $S_{\mathrm{J}}$ cluster 20 and three strains which fell into $S_{\mathrm{J}}$ cluster 19 . The remaining strains forming $S_{\mathrm{J}}$ cluster 20 (five strains) were found in $S_{\mathrm{SM}}$ cluster 9 (S. violaceus). $S_{\mathrm{SM}}$ cluster 9 
Table 4. Distribution of positive characters to single-member subclusters

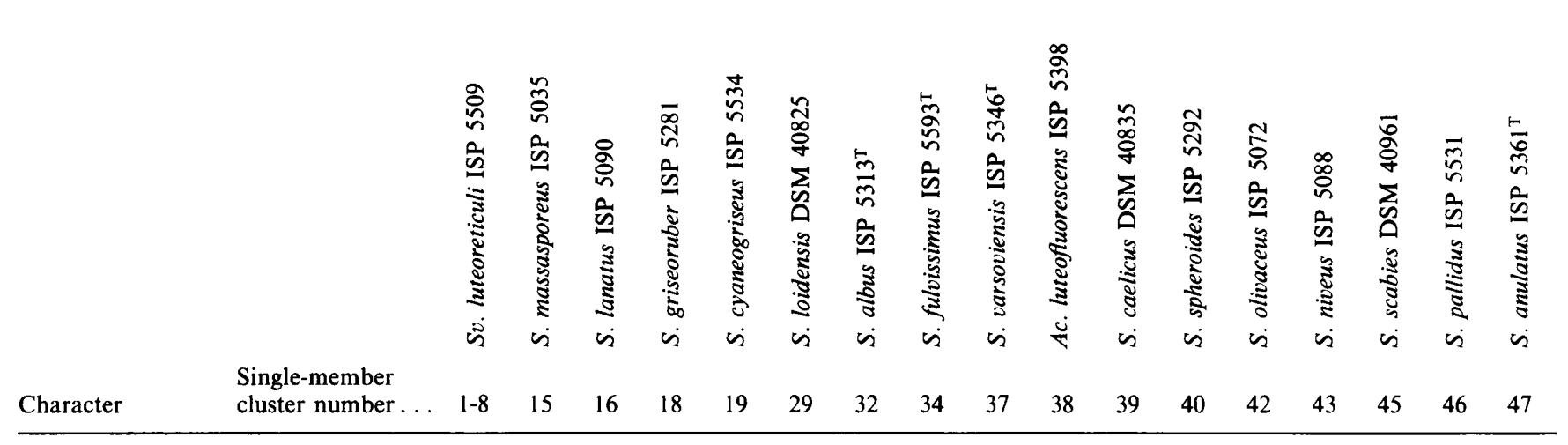

Carbon source used for growth:

Carbohydrates and sugar derivatives

$N$-Acetyl-D-galactosamine

$N$-Acetyl-D-glucosamine

Amygdalin

D-Arabinose

L-Arabinose

Arabonic acid

p-Arbutin

D-Cellobiose

Dextran

D-Fructose

D-Fucose

L-Fucose

D-Galactose

$\alpha$-D-Galacturonate

D-Glucarate

Gluconate

D-Glucosamine

D-Glucosaminic acid

D-Glucose

D-Glucuronate

Glycogen

Glycyrrhizinic acid

Inulin

Lactose

Lactulose

D-Lyxose

L-Lyxose

D-Maltose

D-Mannose

L-Mannose

D-Mannoheptulose

$\alpha$-D-Melezitose

$\alpha$-D-Melibiose

Methyl $\alpha$-D-glucopyranoside

Methyl $\alpha$-D-mannoside

Methyl $\beta$-D-xyloside

Mucate

5-Oxogluconate

$\alpha$-D-Raffinose

L-Rhamnose

D-Ribose

D-Sucrose

Salicin

L-Sorbose

Starch

D-Tagatose

D-Trehalose

D-Turanose

D-Xylose

L-Xylose

\begin{tabular}{|c|c|c|c|c|c|c|c|c|c|c|c|c|c|}
\hline 0 & 1 & 0 & 1 & 1 & 1 & 1 & 0 & 1 & 1 & 1 & 0 & 1 & 1 \\
\hline 1 & 1 & 1 & 1 & 1 & 1 & 1 & 1 & 1 & 1 & 1 & 1 & 1 & 1 \\
\hline 0 & 0 & 0 & 0 & 1 & 0 & 1 & 0 & 0 & 1 & 0 & 0 & 0 & 0 \\
\hline 0 & 0 & 1 & 1 & 0 & 0 & 0 & 0 & 0 & 1 & 1 & 0 & 1 & 0 \\
\hline 1 & 1 & 1 & 1 & 1 & 0 & 1 & 0 & 1 & 1 & 0 & 1 & & 1 \\
\hline 0 & 0 & 0 & 1 & 0 & 0 & 0 & 0 & 0 & 0 & 0 & 0 & ( & 0 \\
\hline 1 & 1 & 1 & 1 & 0 & 1 & 1 & 1 & 0 & 1 & 1 & 1 & 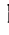 & 1 \\
\hline 1 & 1 & 1 & 1 & 1 & 1 & 1 & 0 & 1 & 1 & 1 & 1 & & 1 \\
\hline 0 & 0 & 0 & 1 & 0 & 0 & 1 & 0 & 0 & 0 & 0 & 1 & & 0 \\
\hline 1 & 1 & 1 & 1 & 1 & 0 & 1 & 1 & 1 & 1 & 1 & 1 & & 1 \\
\hline 0 & 0 & 1 & 0 & 0 & 0 & 1 & 0 & 0 & 0 & 0 & 0 & & 1 \\
\hline 0 & 1 & 1 & 1 & 0 & 0 & 1 & 1 & 0 & 1 & 1 & 0 & & 1 \\
\hline 1 & 1 & 1 & 1 & 1 & 1 & 1 & 1 & 0 & 1 & 1 & 1 & & 1 \\
\hline 0 & 0 & 0 & 1 & 0 & 0 & 1 & 0 & 1 & 1 & 0 & 1 & ( & 0 \\
\hline 0 & 0 & 0 & 1 & 0 & 0 & 1 & 0 & 0 & 1 & 1 & 0 & ( & 1 \\
\hline 1 & 1 & 0 & 0 & 1 & 1 & 1 & 1 & 1 & 1 & 1 & 1 & & 1 \\
\hline 1 & 1 & 1 & 1 & 1 & 0 & 1 & 1 & 1 & 1 & 1 & 1 & & 1 \\
\hline 1 & 0 & 1 & 1 & 1 & 1 & 1 & 1 & 1 & 1 & 1 & 0 & & 1 \\
\hline 1 & 1 & 1 & 1 & 1 & 1 & 1 & 1 & 1 & 1 & 1 & 0 & & 1 \\
\hline 0 & 0 & 0 & 0 & 0 & 0 & 1 & 1 & 0 & 0 & 0 & 1 & ( & 1 \\
\hline 1 & 1 & 1 & 1 & 1 & 0 & 1 & 0 & 1 & 1 & 1 & 1 & & 1 \\
\hline 0 & 0 & 0 & 0 & 0 & 0 & 0 & 0 & 0 & 0 & 1 & 0 & & 0 \\
\hline 0 & 0 & 0 & 0 & 1 & 1 & 0 & 1 & 1 & 0 & 0 & 1 & & 1 \\
\hline 1 & 1 & 1 & 0 & 0 & 1 & 1 & 0 & 0 & 1 & 0 & 1 & & 1 \\
\hline 1 & 1 & 1 & 0 & 1 & 1 & 0 & 0 & 0 & 0 & 0 & 0 & & 1 \\
\hline 0 & 0 & 0 & 0 & 0 & 0 & 0 & 0 & 0 & 0 & 0 & 1 & & 0 \\
\hline 0 & 1 & 1 & 1 & 0 & 0 & 1 & 0 & 1 & 1 & 1 & 0 & & 0 \\
\hline 1 & 1 & 1 & 1 & 1 & 1 & 1 & 0 & 1 & 1 & 0 & 1 & & 1 \\
\hline 1 & 1 & 1 & 1 & 1 & 1 & 1 & 1 & 0 & 1 & 1 & 1 & & 1 \\
\hline 0 & 1 & 1 & 1 & 0 & 0 & 0 & 0 & 1 & 1 & 0 & 0 & & 1 \\
\hline 0 & 0 & 0 & 0 & 0 & 1 & 0 & 0 & 0 & 0 & 0 & 0 & & 0 \\
\hline 1 & 0 & 0 & 0 & 0 & 0 & 0 & 0 & 0 & 0 & 0 & 0 & & 0 \\
\hline 1 & 1 & 1 & 1 & 1 & 0 & 1 & 0 & 0 & 1 & 1 & 0 & & 1 \\
\hline 0 & 0 & 0 & 0 & 0 & 0 & 1 & 0 & 0 & 0 & 0 & 0 & & 0 \\
\hline 0 & 0 & 0 & 0 & 0 & 0 & 0 & 1 & 0 & 0 & 1 & 1 & & 1 \\
\hline 0 & 0 & 0 & 0 & 0 & 0 & 1 & 0 & 0 & 0 & 0 & 0 & & 0 \\
\hline 1 & 1 & 1 & 1 & 1 & 0 & 1 & 1 & 1 & 1 & 1 & 0 & & 1 \\
\hline 1 & 1 & 0 & 0 & 0 & 1 & 0 & 1 & 0 & 1 & 0 & 1 & & 1 \\
\hline 1 & 1 & 0 & 0 & 0 & 0 & 1 & 0 & 0 & 0 & 0 & 0 & & 1 \\
\hline 1 & 1 & 1 & 1 & 0 & 0 & 0 & 0 & 1 & 1 & 1 & 1 & & 1 \\
\hline 1 & 1 & 1 & 1 & 1 & 1 & 1 & 1 & 0 & 1 & 1 & 1 & & 1 \\
\hline 1 & 1 & 0 & 1 & 0 & 1 & 1 & 0 & 0 & 0 & 1 & 1 & & 1 \\
\hline 1 & 1 & 1 & 1 & 0 & 1 & 1 & 1 & 0 & 1 & 1 & 1 & & 1 \\
\hline 0 & 0 & 0 & 0 & 0 & 0 & 0 & 0 & 0 & 0 & 0 & 0 & & 0 \\
\hline 1 & 1 & 1 & 1 & 1 & 0 & 1 & 0 & 1 & 1 & 1 & 1 & & 1 \\
\hline 0 & 0 & 0 & 0 & 0 & 0 & 0 & 0 & 0 & 0 & 0 & 0 & & 0 \\
\hline 1 & 1 & 1 & 0 & 1 & 1 & 1 & 1 & 1 & 1 & 1 & 1 & & 1 \\
\hline 1 & 0 & 0 & 0 & 0 & 1 & 1 & 0 & 0 & 0 & 1 & 1 & & 0 \\
\hline 1 & 1 & 1 & 1 & 1 & 0 & 1 & 0 & 1 & 1 & 0 & & & 0 \\
\hline 0 & 0 & 0 & 0 & 0 & 0 & 0 & 0 & 0 & 0 & 0 & ( & & 0 \\
\hline
\end{tabular}




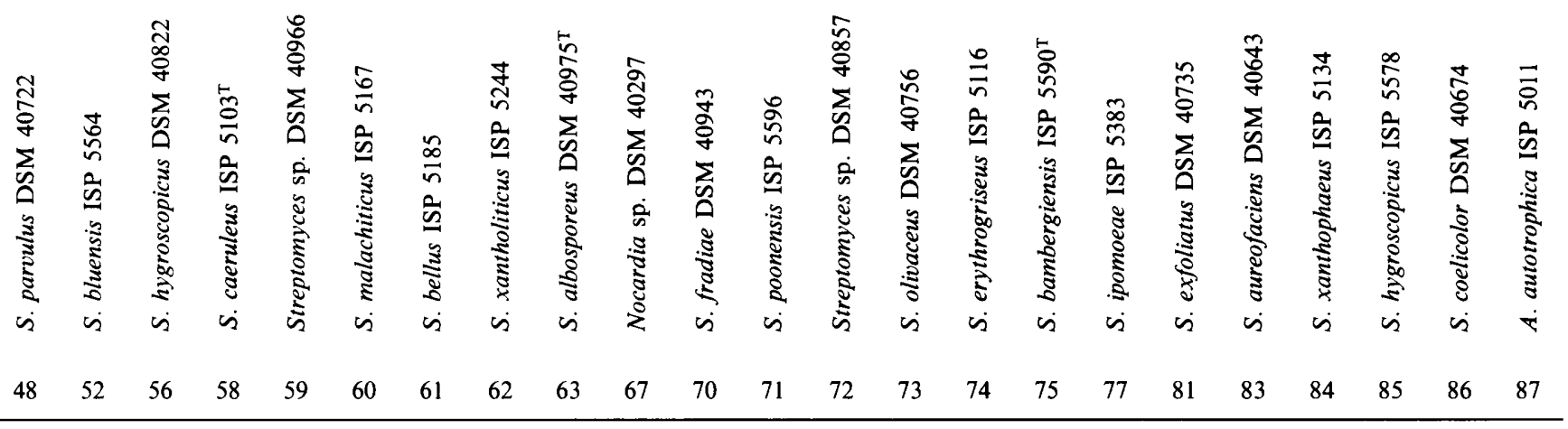

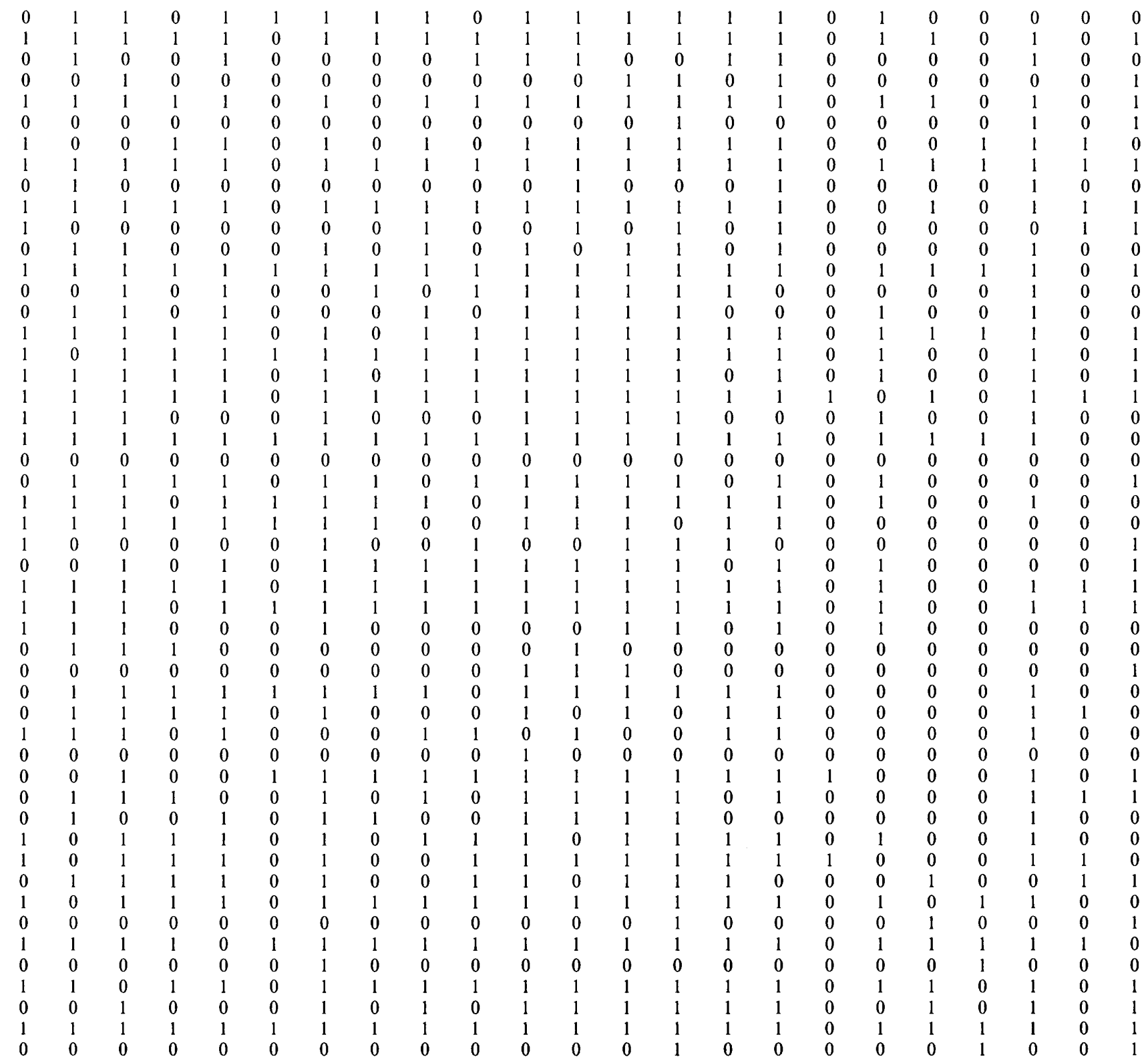


Table 4 (continued)

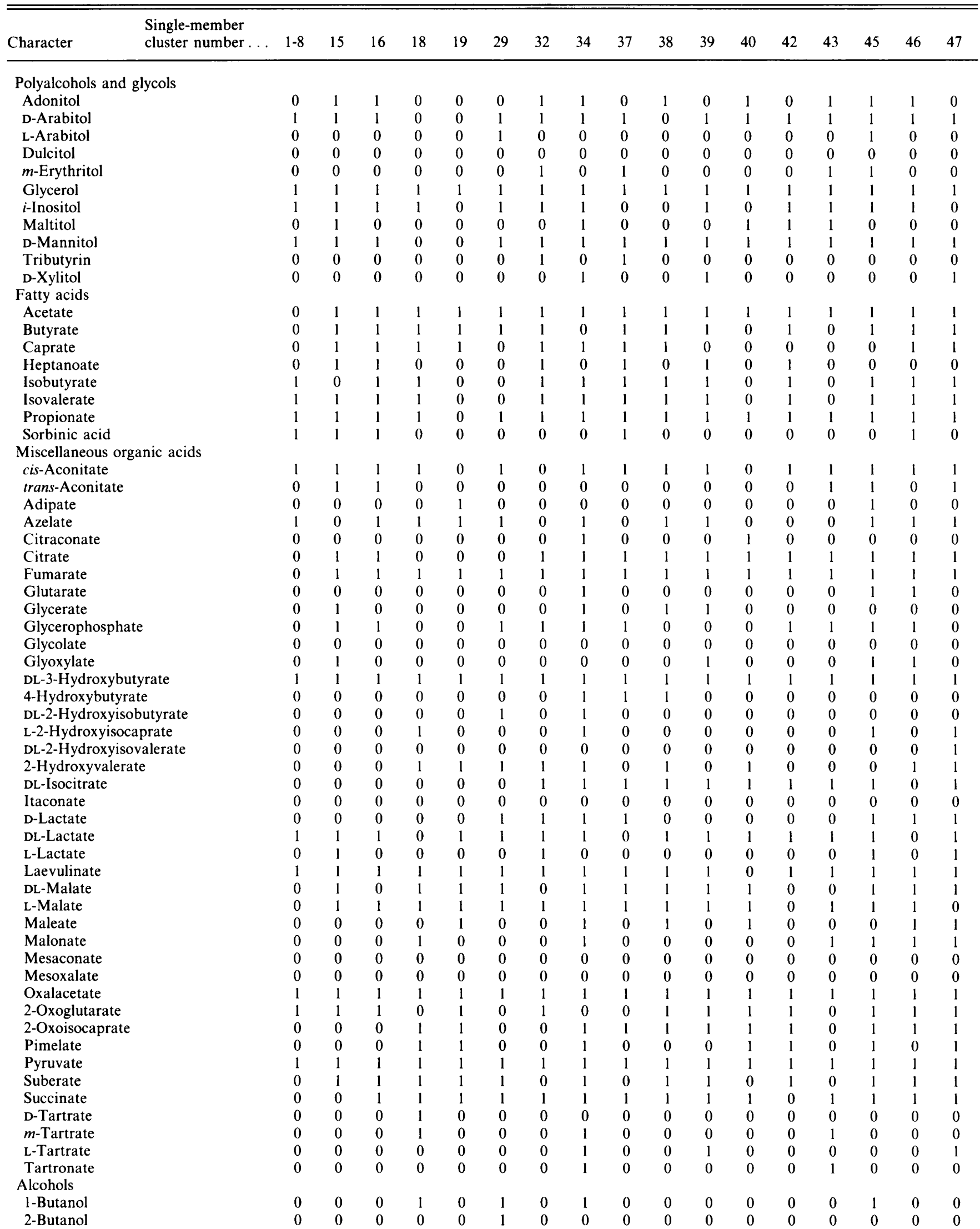




\begin{tabular}{|c|c|c|c|c|c|c|c|c|c|c|c|c|c|c|c|c|c|c|c|c|c|c|}
\hline 48 & 52 & 56 & 58 & 59 & 60 & 61 & 62 & 63 & 67 & 70 & 71 & 72 & 73 & 74 & 75 & 77 & 81 & 83 & 84 & 85 & 86 & 87 \\
\hline 0 & 0 & 1 & 0 & 0 & 0 & 1 & 0 & 0 & 0 & 0 & 0 & 1 & 1 & 0 & 0 & 0 & 0 & 0 & 0 & 1 & 0 & 1 \\
\hline 1 & 1 & 1 & 1 & 0 & 0 & 1 & 0 & 1 & 1 & 0 & 1 & 1 & 1 & 1 & 1 & 0 & 1 & 0 & 0 & 1 & 1 & 1 \\
\hline 0 & 0 & 1 & 0 & 0 & 0 & 0 & 0 & 0 & 1 & 0 & 1 & 1 & 1 & 0 & 0 & 1 & 0 & 0 & 0 & 0 & 0 & 0 \\
\hline 0 & 0 & 0 & 0 & 0 & 0 & 0 & 0 & 1 & 1 & 0 & 0 & 1 & 0 & 0 & 0 & 0 & 0 & 0 & 0 & 0 & 0 & 0 \\
\hline 0 & 0 & 0 & 0 & 1 & 0 & 0 & 0 & 0 & 0 & 0 & 0 & 1 & 1 & 0 & 0 & 0 & 0 & 0 & 0 & 1 & 0 & 1 \\
\hline 1 & 1 & 1 & 1 & 1 & 1 & 1 & 1 & 1 & 1 & 1 & 1 & 1 & 1 & 1 & 1 & 1 & 1 & 1 & 1 & 1 & 0 & 1 \\
\hline 1 & 1 & 1 & 0 & 1 & 1 & 1 & 1 & 1 & 1 & 1 & 1 & 0 & 1 & 1 & 1 & 0 & 0 & 0 & 0 & 1 & 1 & 0 \\
\hline 0 & 1 & 1 & 0 & 0 & 0 & 0 & 0 & 0 & 1 & 1 & 1 & 1 & 1 & 0 & 1 & 0 & 0 & 0 & 0 & 1 & 0 & 1 \\
\hline 1 & 1 & 1 & 1 & 0 & 0 & 1 & 0 & 1 & 1 & 1 & 1 & 1 & 1 & 1 & 1 & 0 & 1 & 0 & 0 & 1 & 0 & 1 \\
\hline 0 & 0 & 1 & 1 & 0 & 0 & 0 & 0 & 0 & 0 & 0 & 0 & 0 & 0 & 0 & 0 & 1 & 0 & 0 & 0 & 0 & 0 & 0 \\
\hline 0 & 0 & 1 & 1 & 1 & 0 & 0 & 0 & 1 & 1 & 1 & 0 & 1 & 1 & 0 & 1 & 0 & 0 & 0 & 0 & 0 & 0 & 1 \\
\hline 1 & 1 & 0 & 1 & 0 & 1 & 1 & 1 & 1 & 1 & 1 & 1 & 1 & 1 & 1 & 1 & 1 & 1 & 1 & 1 & 1 & 1 & 1 \\
\hline 1 & 0 & 1 & 0 & 0 & 0 & 0 & 1 & 1 & 1 & 1 & 1 & 1 & 1 & 1 & 1 & 1 & 1 & 1 & 1 & 1 & 1 & 1 \\
\hline 0 & 0 & 0 & 0 & 0 & 0 & 1 & 0 & 0 & 1 & 0 & 1 & 1 & 1 & 1 & 0 & 1 & 0 & 0 & 0 & 0 & 0 & 1 \\
\hline 1 & 0 & 0 & 0 & 0 & 0 & 1 & 0 & 0 & 1 & 0 & 0 & 1 & 1 & 1 & 0 & 1 & 0 & 0 & 0 & 0 & 0 & 1 \\
\hline 1 & 0 & 1 & 1 & 0 & 0 & 1 & 0 & 1 & 1 & 1 & 1 & 1 & 1 & 1 & 1 & 1 & 1 & 0 & 0 & 0 & 0 & 1 \\
\hline 1 & 0 & 0 & 0 & 0 & 0 & 1 & 1 & 1 & 1 & 1 & 0 & 1 & 1 & 1 & 0 & 1 & 1 & 0 & 1 & 0 & 1 & 1 \\
\hline 1 & 0 & 0 & 1 & 0 & 1 & 1 & 1 & 1 & 1 & 1 & 1 & 1 & 1 & 1 & 1 & 1 & 1 & 1 & 1 & 1 & 0 & 1 \\
\hline 1 & 1 & 0 & 0 & 0 & 0 & 1 & 0 & 0 & 1 & 0 & 0 & 1 & 1 & 1 & 0 & 1 & 0 & 0 & 0 & 0 & 0 & 0 \\
\hline 1 & 1 & 1 & 1 & 0 & 0 & 0 & 1 & 1 & 1 & 1 & 1 & 1 & 1 & 1 & 0 & 0 & 0 & 0 & 0 & 1 & 1 & 1 \\
\hline 0 & 1 & 1 & 1 & 0 & 0 & 0 & 0 & 0 & 0 & 1 & 0 & 1 & 0 & 0 & 0 & 0 & 0 & 0 & 0 & 0 & 0 & 0 \\
\hline 0 & 0 & 0 & 1 & 0 & 0 & 1 & 1 & 1 & 1 & 1 & 1 & 1 & 0 & 1 & 1 & 0 & 0 & 0 & 0 & 0 & 0 & 1 \\
\hline 0 & 0 & 0 & 0 & 0 & 1 & 0 & 1 & 0 & 1 & 1 & 1 & 0 & 1 & 1 & 1 & 0 & 0 & 0 & 0 & 0 & 0 & 1 \\
\hline 0 & 0 & 0 & 0 & 1 & 0 & 0 & 0 & 1 & 0 & 0 & 1 & 0 & 1 & 0 & 0 & 0 & 0 & 0 & 1 & 0 & 0 & 1 \\
\hline 1 & 1 & 0 & 1 & 0 & 0 & 0 & 1 & 0 & 1 & 1 & 1 & 1 & 1 & 1 & 1 & 0 & 0 & 0 & 0 & 1 & 0 & 1 \\
\hline 1 & 1 & 1 & 1 & 1 & 0 & 0 & 1 & 1 & 1 & 1 & 1 & 1 & 1 & 1 & 1 & 1 & 1 & 0 & 1 & 1 & 1 & 1 \\
\hline 0 & 0 & 0 & 0 & 0 & 0 & 0 & 1 & 1 & 1 & 0 & 1 & 0 & 0 & 1 & 1 & 0 & 0 & 1 & 0 & 0 & 0 & 0 \\
\hline 0 & 0 & 0 & 0 & 0 & 0 & 0 & 1 & 1 & 1 & 1 & 1 & 1 & 1 & 1 & 1 & 0 & 0 & 0 & 0 & 0 & 0 & 1 \\
\hline 1 & 1 & 1 & 0 & 0 & 0 & 0 & 0 & 0 & 1 & 0 & 1 & 0 & 1 & 0 & 1 & 0 & 0 & 0 & 0 & 1 & 1 & 0 \\
\hline 0 & 0 & 0 & 0 & 0 & 0 & 0 & 0 & 0 & 0 & 0 & 1 & 0 & 1 & 0 & 0 & 0 & 0 & 0 & 0 & 0 & 0 & 0 \\
\hline 0 & 1 & 0 & 0 & 0 & 0 & 0 & 0 & 1 & 1 & 1 & 1 & 0 & 1 & 1 & 1 & 0 & 0 & 0 & 0 & 0 & 0 & 1 \\
\hline 0 & 1 & 0 & 0 & 1 & 0 & 1 & 1 & 1 & 1 & 1 & 1 & 1 & 1 & 1 & 1 & 1 & 1 & 0 & 1 & 1 & 0 & 1 \\
\hline 0 & 0 & 0 & 0 & 0 & 0 & 0 & 0 & 0 & 0 & 1 & 0 & 1 & 1 & 1 & 1 & 1 & 0 & 0 & 0 & 0 & 0 & 1 \\
\hline 0 & 0 & 0 & 0 & 0 & 0 & 0 & 0 & 0 & 0 & 0 & 1 & 0 & 0 & 0 & 0 & 0 & 0 & 0 & 0 & 0 & 1 & 1 \\
\hline 0 & 0 & 0 & 0 & 0 & 0 & 0 & 1 & 0 & 1 & 1 & 1 & 0 & 0 & 1 & 1 & 1 & 0 & 0 & 0 & 0 & 0 & 1 \\
\hline 0 & 0 & 1 & 0 & 0 & 0 & 0 & 1 & 0 & 1 & 1 & 0 & 1 & 0 & 0 & 0 & 0 & 0 & 0 & 0 & 0 & 0 & 1 \\
\hline 0 & 0 & 1 & 0 & 1 & 0 & 1 & 1 & 1 & 1 & 0 & 1 & 1 & 1 & 0 & 1 & 1 & 0 & 0 & 0 & 0 & 1 & 1 \\
\hline 1 & 0 & 0 & 0 & 0 & 0 & 0 & 0 & 0 & 1 & 1 & 1 & 0 & 1 & 1 & 0 & 0 & 0 & 0 & 0 & 1 & 1 & 0 \\
\hline 0 & 0 & 0 & 0 & 1 & 0 & 0 & 0 & 0 & 0 & 0 & 0 & 0 & 1 & 1 & 0 & 0 & 0 & 0 & 0 & 1 & 0 & 1 \\
\hline 0 & 0 & 1 & 1 & 0 & 0 & 0 & 0 & 1 & 1 & 1 & 1 & 1 & 1 & 1 & 0 & 1 & 0 & 0 & 0 & 0 & 1 & 1 \\
\hline 1 & 1 & 1 & 1 & 1 & 0 & 1 & 1 & 1 & 1 & 1 & 1 & 1 & 1 & 1 & 1 & 1 & 0 & 0 & 1 & 0 & 1 & 1 \\
\hline 0 & 1 & 1 & 0 & 1 & 0 & 0 & 1 & 0 & 1 & 1 & 0 & 1 & 1 & 0 & 1 & 1 & 0 & 0 & 0 & 0 & 0 & 1 \\
\hline 0 & 0 & 1 & 0 & 0 & 1 & 1 & 0 & 1 & 1 & 1 & 1 & 1 & 1 & 1 & 1 & 1 & 1 & 0 & 1 & 1 & 0 & 1 \\
\hline 0 & 1 & 0 & 0 & 1 & 0 & 0 & 1 & 1 & 1 & 1 & 1 & 1 & 1 & 0 & 1 & 1 & 1 & 1 & 0 & 1 & 1 & 0 \\
\hline 1 & 1 & 1 & 1 & 1 & 0 & 0 & 1 & 1 & 1 & 1 & 1 & 1 & 1 & 1 & 1 & 1 & 1 & 1 & 1 & 1 & 1 & 0 \\
\hline 0 & 1 & 0 & 0 & 0 & 0 & 0 & 1 & 1 & 0 & 1 & 1 & 0 & 1 & 0 & 1 & 0 & 0 & 0 & 0 & 0 & 1 & 0 \\
\hline 0 & 1 & 0 & 0 & 0 & 0 & 0 & 0 & 1 & 0 & 1 & 1 & 1 & 0 & 1 & 1 & 0 & 0 & 0 & 1 & 0 & 1 & 0 \\
\hline 0 & 0 & 0 & 0 & 1 & 0 & 0 & 1 & 0 & 0 & 0 & 0 & 0 & 1 & 1 & 1 & 0 & 0 & 0 & 0 & 0 & 0 & 0 \\
\hline 0 & 0 & 0 & 0 & 0 & 0 & 0 & 0 & 0 & 0 & 0 & 1 & 0 & 0 & 1 & 1 & 0 & 0 & 0 & 0 & 0 & 0 & 0 \\
\hline 1 & 1 & 1 & 1 & 1 & 1 & 1 & 1 & 1 & 1 & 1 & 1 & 1 & 1 & 1 & 1 & 1 & 1 & 1 & 1 & 1 & 1 & 1 \\
\hline 1 & 1 & 0 & 1 & 0 & 0 & 0 & 0 & 1 & 1 & 1 & 1 & 1 & 1 & 1 & 1 & 0 & 0 & 0 & 1 & 1 & 0 & 1 \\
\hline 0 & 1 & 0 & 0 & 1 & 0 & 0 & 1 & 1 & 1 & 1 & 1 & 1 & 1 & 1 & 1 & 0 & 0 & 0 & 1 & 0 & 1 & 1 \\
\hline 0 & 0 & 0 & 0 & 0 & 0 & 0 & 1 & 1 & 1 & 0 & 1 & 0 & 0 & 1 & 1 & 0 & 0 & 0 & 0 & 0 & 0 & 0 \\
\hline 1 & 1 & 1 & 1 & 1 & 1 & 1 & 1 & 1 & 1 & 1 & 1 & 1 & 1 & 1 & 1 & 1 & 1 & 1 & 1 & 1 & 1 & 1 \\
\hline 0 & 0 & 0 & 0 & 0 & 0 & 1 & 1 & 1 & 1 & 1 & 1 & 1 & 1 & 1 & 1 & 0 & 1 & 0 & 1 & 0 & 0 & 1 \\
\hline 1 & 1 & 1 & 0 & 1 & 0 & 0 & 0 & 1 & 1 & 1 & 1 & 1 & 1 & 1 & 1 & 1 & 1 & 0 & 1 & 1 & 1 & 0 \\
\hline 1 & 1 & 1 & 0 & 0 & 0 & 1 & 0 & 1 & 0 & 0 & 1 & 0 & 1 & 0 & 1 & 0 & 0 & 0 & 0 & 0 & 1 & 0 \\
\hline 0 & 0 & 1 & 0 & 0 & 0 & 0 & 0 & 1 & 0 & 0 & 0 & 0 & 1 & 1 & 1 & 0 & 0 & 0 & 0 & 0 & 1 & 0 \\
\hline 0 & 1 & 1 & 0 & 0 & 0 & 0 & 0 & 1 & 0 & 1 & 1 & 1 & 0 & 1 & 0 & 0 & 0 & 0 & 0 & 0 & 0 & 0 \\
\hline 0 & 0 & 0 & 0 & 1 & 0 & 0 & 0 & 0 & 0 & 0 & 0 & 0 & 0 & 1 & 0 & 0 & 0 & 0 & 0 & 0 & 0 & 0 \\
\hline 0 & 0 & 0 & 0 & 0 & 0 & 0 & 0 & 0 & 1 & 0 & 0 & 1 & 0 & 0 & 0 & 1 & 0 & 0 & 0 & 0 & 0 & 0 \\
\hline 0 & 0 & 0 & 0 & 0 & 0 & 0 & 0 & 0 & 0 & 0 & 0 & 1 & 0 & 0 & 0 & 1 & 0 & 0 & 0 & 0 & 0 & 0 \\
\hline
\end{tabular}


Table 4 (continued)

\begin{tabular}{|c|c|c|c|c|c|c|c|c|c|c|c|c|c|c|c|c|c|}
\hline $\begin{array}{l}\text { Single-member } \\
\text { cluster number . . . }\end{array}$ & $1-8$ & 15 & 16 & 18 & 19 & 29 & 32 & 34 & 37 & 38 & 39 & 40 & 42 & 43 & 45 & 46 & 47 \\
\hline Ethanol & 0 & 0 & 0 & 1 & 0 & 1 & 0 & 1 & 0 & 0 & 0 & 0 & 0 & 0 & 1 & 0 & 1 \\
\hline 1-Hexanol & 0 & 0 & 0 & 0 & 0 & 1 & 0 & 1 & 0 & 0 & 0 & 0 & 0 & 0 & 0 & 0 & 0 \\
\hline 1,8-Octandiol & 1 & 0 & 0 & 1 & 1 & 1 & 0 & 1 & 1 & 1 & 1 & 1 & 0 & 1 & 1 & 1 & 1 \\
\hline \multicolumn{18}{|l|}{ Amino acids and related compounds } \\
\hline Acetamidocaprate & 0 & 0 & 1 & 1 & 0 & 1 & 0 & 1 & 0 & 1 & 0 & 0 & 1 & 1 & 0 & 1 & 0 \\
\hline Acetyl-L-glutamate & 0 & 0 & 1 & 0 & 0 & 1 & 1 & 1 & 1 & 0 & 1 & 0 & 0 & 1 & 1 & 1 & 1 \\
\hline Acetyl-L-glutamine & 1 & 0 & 1 & 0 & 0 & 1 & 1 & 1 & 1 & 1 & 1 & 1 & 0 & 1 & 1 & 1 & 0 \\
\hline Acetylglycine & 0 & 0 & 0 & 0 & 0 & 1 & 0 & 0 & 0 & 1 & 1 & 1 & 0 & 0 & 1 & 1 & 0 \\
\hline Acetyl-DL-methionine & 0 & 0 & 0 & 0 & 0 & 0 & 0 & 1 & 1 & 0 & 0 & 1 & 0 & 0 & 1 & 0 & 0 \\
\hline Acetyl-L-proline & 0 & 0 & 0 & 0 & 0 & 1 & 0 & 1 & 0 & 0 & 0 & 0 & 0 & 1 & 0 & 0 & 0 \\
\hline D-Alanine & 1 & 1 & 1 & 0 & 1 & 0 & 1 & 1 & 0 & 1 & 0 & 1 & 1 & 0 & 1 & 0 & 0 \\
\hline L-Alanine & 0 & 1 & 1 & 1 & 1 & 1 & 1 & 1 & 1 & 1 & 1 & 0 & 1 & 1 & 1 & 1 & 1 \\
\hline$\beta$-Alanine & 1 & 1 & 1 & 1 & 1 & 1 & 1 & 1 & 1 & 0 & 1 & 0 & 1 & 1 & 1 & 1 & 1 \\
\hline DL-2-Aminoadipate & 1 & 0 & 0 & 1 & 1 & 0 & 0 & 0 & 0 & 0 & 0 & 0 & 0 & 0 & 0 & 1 & 0 \\
\hline DL-2-Aminobutyrate & 0 & 0 & 0 & 1 & 0 & 0 & 0 & 0 & 0 & 0 & 0 & 0 & 0 & 1 & 1 & 1 & 0 \\
\hline DL-3-Aminobutyrate & 1 & 0 & 0 & 0 & 0 & 1 & 0 & 1 & 0 & 0 & 0 & 0 & 0 & 0 & 0 & 1 & 0 \\
\hline DL-2-Aminoisobutyrate & 0 & 0 & 0 & 0 & 0 & 0 & 0 & 0 & 0 & 0 & 0 & 0 & 0 & 0 & 0 & 0 & 0 \\
\hline 4-Aminobutyrate & 1 & 1 & 1 & 1 & 1 & 1 & 0 & 1 & 1 & 1 & 1 & 0 & 0 & 0 & 1 & 1 & 1 \\
\hline Aminoxyacetate & 0 & 0 & 0 & 0 & 0 & 0 & 0 & 0 & 0 & 0 & 0 & 0 & 0 & 0 & 0 & 0 & 0 \\
\hline D-Arginine & 0 & 0 & 1 & 0 & 1 & 0 & 0 & 1 & 0 & 0 & 0 & 0 & 0 & 0 & 0 & 0 & 0 \\
\hline L-Arginine & 1 & 1 & 1 & 1 & 1 & 1 & 1 & 1 & 1 & 1 & 1 & 1 & 0 & 0 & 1 & 1 & 0 \\
\hline D-Asparagine & 0 & 0 & 0 & 0 & 0 & 0 & 0 & 0 & 0 & 0 & 0 & 0 & 0 & 0 & 0 & 0 & 0 \\
\hline L-Asparagine & 1 & 1 & 1 & 1 & 1 & 1 & 1 & 1 & 1 & 0 & 1 & 1 & 1 & 1 & 1 & 1 & 1 \\
\hline L-Aspartate & 0 & 1 & 1 & 1 & 1 & 1 & 1 & 1 & 1 & 1 & 1 & 1 & 1 & 1 & 1 & 1 & 1 \\
\hline DL-Aspartate & 1 & 1 & 0 & 0 & 1 & 1 & 0 & 1 & 1 & 1 & 1 & 1 & 0 & 0 & 0 & 1 & 0 \\
\hline Betaine & 0 & 0 & 0 & 1 & 0 & 0 & 0 & 0 & 0 & 0 & 1 & 1 & 0 & 0 & 0 & 0 & 1 \\
\hline Casein & 1 & 1 & 1 & 1 & 1 & 1 & 1 & 1 & 1 & 1 & 1 & 1 & 1 & 1 & 1 & 1 & 1 \\
\hline L-Cysteinate & 0 & 0 & 0 & 0 & 0 & 0 & 0 & 0 & 0 & 0 & 0 & 1 & 0 & 0 & 0 & 0 & 0 \\
\hline L-Citrulline & 1 & 1 & 1 & 1 & 1 & 0 & 1 & 0 & 0 & 1 & 1 & 0 & 0 & 0 & 1 & 1 & 0 \\
\hline DL-2,4-Diaminobutyrate & 0 & 0 & 0 & 0 & 0 & 0 & 0 & 0 & 0 & 0 & 0 & 0 & 0 & 0 & 0 & 0 & 0 \\
\hline Diaminopimelate & 0 & 0 & 0 & 0 & 1 & 0 & 0 & 0 & 0 & 0 & 0 & 0 & 0 & 0 & 0 & 0 & 0 \\
\hline 2,3-Diaminopropionate & 0 & 0 & 0 & 0 & 0 & 1 & 0 & 1 & 1 & 0 & 0 & 0 & 0 & 0 & 0 & 0 & 0 \\
\hline Dimethylglycine & 0 & 0 & 1 & 0 & 0 & 0 & 0 & 0 & 0 & 0 & 0 & 0 & 0 & 0 & 0 & 0 & 1 \\
\hline D-Glutamate & 0 & 1 & 0 & 1 & 1 & 1 & 1 & 1 & 0 & 0 & 1 & 0 & 0 & 1 & 1 & 1 & 0 \\
\hline L-Glutamate & 0 & 1 & 1 & 1 & 1 & 1 & 1 & 1 & 1 & 1 & 1 & 1 & 1 & 1 & 1 & 1 & 1 \\
\hline L-Glutamine & 1 & 1 & 1 & 1 & 1 & 1 & 1 & 1 & 1 & 1 & 1 & 1 & 1 & 1 & 1 & 1 & 1 \\
\hline L-Glycine & 1 & 1 & 1 & 1 & 0 & 1 & 1 & 1 & 1 & 0 & 1 & 0 & 1 & 1 & 1 & 1 & 1 \\
\hline Glycine anhydride & 0 & 0 & 0 & 0 & 0 & 0 & 0 & 0 & 0 & 0 & 1 & 0 & 0 & 0 & 0 & 0 & 0 \\
\hline D-Histidine & 0 & 0 & 0 & 0 & 0 & 0 & 0 & 1 & 0 & 0 & 0 & 0 & 0 & 0 & 0 & 0 & 0 \\
\hline L-Histidine & 1 & 1 & 1 & 1 & 1 & 1 & 1 & 1 & 1 & 1 & 1 & 1 & 1 & 1 & 1 & 1 & 0 \\
\hline L-Homoserine & 0 & 1 & 0 & 1 & 0 & 1 & 0 & 1 & 0 & 0 & 0 & 0 & 0 & 0 & 1 & 0 & 0 \\
\hline DL- $\delta$-Hydroxylysine & 0 & 0 & 0 & 0 & 0 & 0 & 0 & 0 & 0 & 0 & 0 & 0 & 0 & 0 & 0 & 0 & 0 \\
\hline L-Hydroxyproline & 1 & 1 & 1 & 1 & 1 & 1 & 1 & 1 & 0 & 1 & 0 & 1 & 1 & 1 & 1 & 1 & 1 \\
\hline DL-Isoleucine & 1 & 1 & 0 & 1 & 1 & 0 & 0 & 1 & 0 & 1 & 0 & 0 & 0 & 0 & 1 & 1 & 0 \\
\hline L-Isoleucine & 1 & 1 & 0 & 1 & 1 & 0 & 1 & 1 & 1 & 1 & 0 & 0 & 0 & 0 & 1 & 1 & 1 \\
\hline D-Leucine & 0 & 0 & 0 & 0 & 0 & 0 & 0 & 1 & 0 & 0 & 0 & 0 & 0 & 0 & 1 & 0 & 0 \\
\hline L-Leucine & 0 & 0 & 0 & 0 & 1 & 1 & 0 & 1 & 1 & 1 & 1 & 1 & 0 & 0 & 1 & 1 & 1 \\
\hline D-Lysine & 0 & 0 & 1 & 0 & 0 & 0 & 0 & 0 & 0 & 0 & 0 & 0 & 0 & 0 & 0 & 0 & 0 \\
\hline L-Lysine & 1 & 1 & 1 & 1 & 1 & 1 & 1 & 1 & 1 & 0 & 1 & 1 & 0 & 1 & 0 & 0 & 0 \\
\hline D-Methionine & 0 & 0 & 0 & 0 & 0 & 0 & 0 & 0 & 0 & 0 & 0 & 0 & 0 & 0 & 0 & 0 & 0 \\
\hline L-Methionine & 0 & 0 & 0 & 0 & 0 & 0 & 0 & 0 & 0 & 0 & 0 & 0 & 0 & 0 & 0 & 0 & 0 \\
\hline DL-Methioninesulphone & 0 & 0 & 0 & 0 & 0 & 0 & 0 & 0 & 0 & 0 & 0 & 0 & 0 & 0 & 0 & 0 & 0 \\
\hline L-Norleucine & 0 & 0 & 0 & 0 & 0 & 0 & 0 & 0 & 0 & 0 & 0 & 0 & 0 & 0 & 1 & 1 & 1 \\
\hline D-Norvaline & 0 & 0 & 0 & 1 & 0 & 0 & 0 & 1 & 0 & 1 & 0 & 0 & 0 & 0 & 0 & 0 & 0 \\
\hline L-Norvaline & 0 & 0 & 0 & 0 & 0 & 0 & 0 & 1 & 0 & 0 & 0 & 0 & 0 & 0 & 0 & 1 & 0 \\
\hline L-Ornithine & 1 & 1 & 1 & 1 & 1 & 1 & 1 & 1 & 1 & 1 & 1 & 1 & 0 & 0 & 1 & 1 & 0 \\
\hline D-Phenylalanine & 0 & 1 & 1 & 0 & 0 & 0 & 0 & 1 & 1 & 1 & 0 & 0 & 0 & 0 & 1 & 0 & 1 \\
\hline L-Phenylalanine & 1 & 1 & 1 & 1 & 1 & 1 & 1 & 1 & 1 & 1 & 1 & 1 & 1 & 1 & 1 & 1 & 1 \\
\hline Phenylglycine & 0 & 0 & 0 & 0 & 0 & 0 & 0 & 0 & 0 & 0 & 0 & 0 & 0 & 0 & 0 & 0 & 0 \\
\hline D-Proline & 0 & 0 & 0 & 0 & 1 & 0 & 0 & 1 & 0 & 1 & 0 & 0 & 0 & 0 & 0 & 1 & 0 \\
\hline L-Proline & 1 & 1 & 1 & 1 & 1 & 1 & 1 & 1 & 1 & 1 & 1 & 1 & 1 & 1 & 1 & 1 & 1 \\
\hline Sarcosine & 0 & 0 & 0 & 1 & 0 & 0 & 0 & 0 & 0 & 0 & 0 & 0 & 0 & 0 & 0 & 0 & 0 \\
\hline D-Serine & 0 & 1 & 1 & 0 & 0 & 0 & 0 & 1 & 0 & 0 & 0 & 0 & 0 & 0 & 0 & 0 & 0 \\
\hline L-Serine & 1 & 1 & 1 & 1 & 1 & 1 & 1 & 1 & 1 & 1 & 1 & 1 & 1 & 1 & 1 & 1 & 1 \\
\hline L-Threonine & 0 & 1 & 1 & 1 & 1 & 1 & 1 & 1 & 0 & 1 & 1 & 0 & 1 & 1 & 1 & 1 & 1 \\
\hline D-Tryptophan & 0 & 0 & 0 & 1 & 0 & 0 & 0 & 0 & 0 & 0 & 0 & 0 & 0 & 0 & 0 & 0 & 0 \\
\hline
\end{tabular}




\section{$\begin{array}{llllllllllllllllllllllll}48 & 52 & 56 & 58 & 59 & 60 & 61 & 62 & 63 & 67 & 70 & 71 & 72 & 73 & 74 & 75 & 77 & 81 & 83 & 84 & 85 & 86 & 87\end{array}$}

$\begin{array}{lllllllllllllllllllllllllllll}1 & 0 & 0 & 0 & 0 & 0 & 0 & 0 & 0 & 0 & 0 & 0 & 0 & 0 & 0 & 0 & 0 & 0 & 0 & 0 & 0 & 0 & 1 \\ 0 & 0 & 0 & 0 & 0 & 0 & 0 & 0 & 0 & 0 & 1 & 0 & 1 & 0 & 0 & 0 & 1 & 0 & 0 & 0 & 0 & 0 & 0 \\ 1 & 0 & 0 & 0 & 0 & 0 & 1 & 1 & 1 & 1 & 1 & 1 & 1 & 0 & 0 & 1 & 1 & 0 & 0 & 0 & 0\end{array}$

\begin{tabular}{|c|c|c|c|c|c|c|c|c|c|c|c|c|c|c|c|c|c|c|c|c|c|}
\hline 0 & 1 & 1 & 0 & 0 & 1 & 1 & 1 & 1 & 1 & 1 & 1 & 1 & 1 & 1 & 1 & 0 & 0 & 0 & 0 & 1 & 0 \\
\hline 0 & 1 & 1 & 1 & 1 & 0 & 0 & 1 & 1 & 1 & 1 & 1 & 1 & 0 & 1 & 0 & 1 & 1 & 0 & 0 & 0 & 0 \\
\hline 0 & 1 & 0 & 1 & 1 & 0 & 0 & 1 & 1 & 1 & 1 & 1 & 0 & 1 & 1 & 0 & 0 & 0 & 1 & 0 & 0 & 1 \\
\hline 1 & 1 & 0 & 0 & 0 & 0 & 1 & 1 & 0 & 1 & 1 & 1 & 1 & 1 & 1 & 0 & 1 & 1 & 0 & 0 & 1 & 1 \\
\hline 0 & 0 & 0 & 0 & 0 & 0 & 0 & 0 & 0 & 0 & 0 & 0 & 0 & 0 & 0 & 0 & 1 & 0 & 0 & 0 & 0 & 1 \\
\hline 0 & 0 & 0 & 0 & 0 & 0 & 0 & 0 & 0 & 1 & 1 & 0 & 1 & 0 & 0 & 0 & 1 & 0 & 0 & 0 & 0 & 0 \\
\hline 1 & 1 & 0 & 0 & 1 & 1 & 1 & 1 & 1 & 1 & 1 & 1 & 1 & 0 & 1 & 1 & $\mathbf{0}$ & 0 & 0 & 1 & 1 & 0 \\
\hline 1 & 1 & 1 & 0 & 1 & 1 & 1 & 0 & 1 & 1 & 1 & 1 & 1 & 1 & 1 & 1 & 0 & 1 & 1 & 1 & 1 & 1 \\
\hline 0 & 0 & 0 & 0 & 0 & 1 & 1 & 1 & 0 & 1 & 1 & 1 & 1 & 1 & 1 & 1 & 0 & 0 & 0 & 1 & 1 & 1 \\
\hline 0 & 0 & 0 & 0 & 0 & 0 & 1 & 1 & 0 & 1 & 0 & 0 & 0 & 1 & 1 & 1 & 1 & 0 & 0 & 0 & 0 & 0 \\
\hline 0 & 0 & 0 & 1 & 0 & 0 & 1 & 1 & 0 & 1 & 0 & 0 & 1 & 0 & 0 & 1 & 0 & 0 & 0 & 0 & 1 & 1 \\
\hline 1 & 0 & 0 & 0 & 0 & 0 & 0 & 0 & 0 & 1 & 0 & 0 & 0 & 1 & 0 & 0 & 0 & 0 & 0 & 0 & 0 & 1 \\
\hline 0 & 0 & 0 & 0 & 0 & 0 & 0 & 0 & 0 & 0 & 0 & 0 & 0 & 0 & 0 & 0 & 0 & 0 & 0 & 0 & 0 & 0 \\
\hline 1 & 1 & 1 & 0 & 0 & 1 & 1 & 1 & 1 & 1 & 1 & 1 & 1 & 1 & 1 & 1 & 0 & 0 & 1 & 0 & 1 & 0 \\
\hline 0 & 0 & 0 & 0 & 0 & 0 & 0 & 0 & 0 & 0 & 0 & 0 & 0 & 0 & 0 & 0 & 0 & 0 & 0 & 0 & 1 & 0 \\
\hline 0 & 0 & 0 & 0 & 0 & 0 & 0 & 0 & 0 & 0 & 1 & 0 & 0 & 0 & 0 & 0 & 0 & 0 & 1 & 0 & 0 & 0 \\
\hline 1 & 1 & 1 & 0 & 0 & 1 & 1 & 1 & 1 & 1 & 1 & 0 & 1 & 1 & 1 & 1 & 0 & 0 & 0 & 1 & 1 & 1 \\
\hline 0 & 1 & 0 & 0 & 0 & 0 & 0 & 0 & 0 & 0 & 0 & 1 & 0 & 0 & 0 & 1 & 0 & 0 & 0 & 0 & 0 & 0 \\
\hline 1 & 0 & 0 & 1 & 1 & 0 & 1 & 1 & 1 & 0 & 1 & 1 & 1 & 1 & 1 & 1 & 0 & 0 & 0 & 1 & 1 & 0 \\
\hline 1 & 1 & 1 & 1 & 1 & 1 & 1 & 1 & 1 & 1 & 1 & 1 & 1 & 1 & 1 & 1 & 0 & 1 & 0 & 1 & 1 & 1 \\
\hline 1 & 1 & 1 & 1 & 1 & 0 & 0 & 1 & 1 & 1 & 1 & 1 & 1 & 1 & 1 & 1 & 0 & 1 & 1 & 0 & 0 & 1 \\
\hline 0 & 0 & 0 & 0 & 0 & 1 & 1 & 0 & 0 & 0 & 1 & 0 & 0 & 1 & 1 & 0 & 1 & 0 & 0 & 0 & 1 & 0 \\
\hline 1 & 1 & 1 & 1 & 1 & 1 & 1 & 1 & 1 & 1 & 1 & 1 & 1 & 1 & 1 & 1 & 0 & 1 & 1 & 1 & 1 & 0 \\
\hline 0 & 0 & 0 & 0 & 0 & 0 & 0 & 0 & 1 & 0 & 0 & 0 & 0 & 0 & 0 & 0 & 0 & 0 & 0 & 0 & 0 & 0 \\
\hline 1 & 0 & 0 & 0 & 0 & 1 & 1 & 1 & 0 & 1 & 1 & 1 & 0 & 1 & 1 & 1 & 0 & 1 & 0 & 0 & 1 & 0 \\
\hline 1 & 0 & 0 & 0 & 0 & 0 & 0 & 0 & 0 & 0 & 0 & 0 & 0 & 1 & 1 & 0 & 0 & 0 & 0 & 0 & 0 & 1 \\
\hline 0 & 0 & 0 & 0 & 0 & 0 & 0 & 0 & 0 & 0 & 1 & 0 & 0 & 0 & 1 & 0 & 0 & 0 & 0 & 0 & 0 & 0 \\
\hline 0 & 0 & 0 & 0 & 0 & 0 & 0 & 0 & 0 & 0 & 0 & 0 & 1 & 0 & 1 & 0 & 0 & 0 & 0 & 0 & 1 & 0 \\
\hline 0 & 0 & 0 & 0 & 0 & 0 & 0 & 0 & 0 & 0 & 1 & 0 & 1 & 1 & 1 & 0 & 0 & 0 & 0 & 0 & 0 & 0 \\
\hline 1 & 0 & 0 & 0 & 0 & 0 & 0 & 1 & 0 & 0 & 0 & 0 & 1 & 1 & 1 & 0 & 0 & 0 & 0 & 0 & 1 & 0 \\
\hline 1 & 1 & 0 & 1 & 1 & 1 & 1 & 1 & 1 & 1 & 1 & 1 & 1 & 1 & 1 & 1 & 1 & 1 & 1 & 0 & 1 & 0 \\
\hline 1 & 1 & 1 & 1 & 1 & 1 & 1 & 1 & 1 & 1 & 1 & 1 & 1 & 1 & 1 & 1 & 1 & 1 & 1 & 1 & 1 & 0 \\
\hline 0 & 1 & 0 & 0 & 0 & 1 & 1 & 0 & 1 & 1 & 0 & 1 & 1 & 0 & 1 & 1 & 0 & 0 & 0 & 1 & 1 & 1 \\
\hline 0 & 0 & 0 & 0 & 0 & 0 & 0 & 0 & 0 & 0 & 0 & 0 & 0 & 0 & 0 & 0 & 0 & 0 & 0 & 0 & 0 & 0 \\
\hline 0 & 0 & 0 & 0 & 0 & 0 & 0 & 0 & 0 & 1 & 0 & 0 & 0 & 0 & 0 & 0 & 0 & 0 & 0 & 0 & 0 & 0 \\
\hline 1 & 1 & 1 & 1 & 1 & 1 & 1 & 1 & 1 & 1 & 1 & 1 & 1 & 1 & 1 & 1 & 1 & 0 & 0 & 1 & 1 & 0 \\
\hline I & 0 & 1 & 0 & 0 & 0 & 0 & 0 & 0 & 0 & 1 & 1 & 1 & 0 & 0 & 1 & 0 & 0 & 0 & 0 & 0 & 1 \\
\hline 0 & 0 & 0 & 0 & 0 & 0 & 0 & 0 & 1 & 0 & 0 & 0 & 0 & 0 & 0 & 0 & 0 & 0 & 0 & 0 & 0 & 0 \\
\hline
\end{tabular}

$\begin{array}{lllllllllllllllllllllllll}0 & 0 & 0 & 0 & 0 & 1 & 1 & 1 & 1 & 1 & 0 & 0 & 0 & 1 & 0 & 0 & 0 & 0 & 1 & 1 & 1 & 0 & 0\end{array}$

$\begin{array}{llllllllllllllllllllllllllll}1 & 0 & 0 & 1 & 0 & 1 & 0 & 1 & 1 & 1 & 1 & 1 & 0 & 1 & 1 & 1 & 0 & 0 & 1 & 0 & 1 & 0 & 1\end{array}$

$\begin{array}{lllllllllllllllllllllll}1 & 0 & 1 & 0 & 0 & 1 & 1 & 1 & 1 & 1 & 1 & 1 & 1 & 1 & 1 & 1 & 0 & 0 & 0 & 0 & 1 & 0 & 1 \\ 1 & 0 & 0 & 0 & 0 & 0 & 0 & 0 & 0 & 1 & 0 & 0 & 0 & 0 & 0 & 1 & 0 & 0 & 0 & 0 & 0 & 1 & 1\end{array}$

$\begin{array}{lllllllllllllllllllllll}0 & 0 & 1 & 0 & 0 & 1 & 1 & 1 & 1 & 1 & 1 & 1 & 1 & 1 & 1 & 1 & 0 & 0 & 0 & 0 & 1 & 1 & 1\end{array}$

$\begin{array}{lllllllllllllllllllllll}0 & 0 & 0 & 0 & 0 & 0 & 0 & 0 & 0 & 0 & 0 & 0 & 0 & 0 & 0 & 0 & 0 & 1 & 0 & 0 & 0 & 0 & 0\end{array}$

$\begin{array}{lllllllllllllllllllllll}1 & 0 & 0 & 0 & 0 & 0 & 0 & 0 & 0 & 0 & 0 & 0 & 0 & 0 & 0 & 0 & 0 & 0 & 0 & 0 & 0 & 0 & 0\end{array}$

$\begin{array}{lllllllllllllllllllllll}0 & 0 & 0 & 0 & 0 & 0 & 0 & 0 & 0 & 1 & 0 & 0 & 0 & 0 & 1 & 1 & 0 & 0 & 0 & 0 & 0 & 0 & 0 \\ 0 & 0 & 0 & 0 & 0 & 0 & 0 & 0 & 0 & 0 & 0 & 0 & 0 & 0 & 0 & 1 & 0 & 0 & 0 & 0 & 0 & 0 & 0\end{array}$

$\begin{array}{lllllllllllllllllllllllllll}0 & 0 & 1 & 0 & 0 & 0 & 0 & 1 & 0 & 1 & 0 & 1 & 1 & 0 & 1 & 1 & 0 & 0 & 0 & 0 & 1 & 0 & 0\end{array}$

$\begin{array}{llllllllllllllllllllllllll}1 & 0 & 0 & 0 & 0 & 0 & 0 & 1 & 0 & 1 & 0 & 0 & 1 & 0 & 0 & 1 & 0 & 0 & 0 & 0 & 0 & 1 & 1 \\ 0 & 0 & 1 & 0 & 1 & 0 & 0 & 1 & 0 & 1 & 1 & 1 & 1 & 1 & 0 & 1 & 1 & 0 & 0 & 0 & 0 & 1 & 0\end{array}$

$\begin{array}{llllllllllllllllllllllll}0 & 1 & 1 & 1 & 0 & 0 & 1 & 1 & 1 & 1 & 1 & 1 & 1 & 1 & 1 & 0 & 1 & 1 & 0 & 0 & 0 & 0 & 1 & 0 \\ 1 & 0 & 0 & 0 & 0 & 0 & 0 & 1 & 1 & 1 & 0 & 0 & 1 & 0 & 1 & 1 & 0 & 0 & 0 & 0 & 1 & 1 & 1\end{array}$

$\begin{array}{llllllllllllllllllllllllllllll}1 & 1 & 1 & 1 & 1 & 1 & 1 & 1 & 1 & 1 & 1 & 1 & 1 & 1 & 1 & 1 & 0 & 1 & 1 & 1 & 1 & 1 & 0 \\ 0 & 0 & 0 & 0 & 0 & 0 & 0 & 0 & 0 & 0 & 0 & 0 & 0 & 0 & 0 & 0 & 0 & 0 & 0 & 0 & 0 & 0 & 0\end{array}$

$\begin{array}{llllllllllllllllllllllll}0 & 0 & 0 & 0 & 0 & 0 & 0 & 0 & 0 & 0 & 0 & 0 & 0 & 1 & 0 & 0 & 0 & 0 & 0 & 0 & 0 & 0 & 0\end{array}$

$\begin{array}{lllllllllllllllllllllll}1 & 1 & 1 & 1 & 1 & 1 & 1 & 1 & 1 & 1 & 1 & 1 & 1 & 1 & 1 & 1 & 0 & 1 & 1 & 1 & 1 & 1 & 1 \\ 0 & 1 & 1 & 0 & 0 & 0 & 0 & 0 & 0 & 0 & 1 & 0 & 0 & 1 & 1 & 0 & 0 & 0 & 0 & 0 & 1 & 0 & 1\end{array}$

$\begin{array}{lllllllllllllllllllllll}1 & 0 & 0 & 0 & 0 & 0 & 0 & 0 & 0 & 1 & 1 & 0 & 0 & 0 & 0 & 1 & 0 & 0 & 0 & 0 & 0 & 0 & 0\end{array}$

$\begin{array}{lllllllllllllllllllllll}1 & 1 & 0 & 1 & 1 & 0 & 1 & 1 & 1 & 1 & 1 & 1 & 1 & 1 & 1 & 1 & 0 & 0 & 0 & 1 & 1 & 1 & 1 \\ 1 & 1 & 1 & 1 & 1 & 1 & 1 & 1 & 1 & 1 & 1 & 1 & 1 & 1 & 1 & 1 & 0 & 1 & 0 & 1 & 1 & 1 & 1\end{array}$ $\begin{array}{llllllllllllllllllllllllll}1 & 1 & 1 & 1 & 1 & 1 & 1 & 1 & 1 & 1 & 1 & 1 & 1 & 1 & 1 & 1 & 0 & 1 & 0 & 1 & 1 & 1 & 1 \\ 0 & 0 & 0 & 0 & 0 & 0 & 0 & 0 & 0 & 1 & 0 & 0 & 0 & 0 & 0 & 1 & 0 & 0 & 0 & 0 & 1 & 0 & 1\end{array}$ 
Table 4 (continued)

\begin{tabular}{|c|c|c|c|c|c|c|c|c|c|c|c|c|c|c|c|c|c|}
\hline $\begin{array}{l}\text { Single-member } \\
\text { cluster number } \ldots\end{array}$ & $1-8$ & 15 & 16 & 18 & 19 & 29 & 32 & 34 & 37 & 38 & 39 & 40 & 42 & 43 & 45 & 46 & 47 \\
\hline L-Tryptophan & 1 & 1 & 1 & 1 & 1 & 1 & 0 & 1 & 0 & 0 & 0 & 0 & 1 & 1 & 1 & 1 & 0 \\
\hline L-Valine & 1 & 1 & 1 & 1 & 1 & 1 & 1 & 1 & 1 & 1 & 1 & 1 & 1 & 1 & 1 & 1 & 1 \\
\hline \multicolumn{18}{|l|}{ Amines } \\
\hline Butylamine & 0 & 0 & 0 & 0 & 0 & 0 & 0 & 0 & 0 & 0 & 0 & 0 & 0 & 0 & 0 & 0 & 0 \\
\hline Cadaverine & 1 & 0 & 1 & 1 & 0 & 1 & 0 & 1 & 0 & 0 & 1 & 0 & 0 & 0 & 0 & 1 & 0 \\
\hline Carnitine & 0 & 0 & 0 & 0 & 0 & 0 & 0 & 0 & 1 & 0 & 0 & 0 & 0 & 0 & 0 & 0 & 0 \\
\hline Creatine & 0 & 0 & 0 & 0 & 0 & 0 & 0 & 0 & 0 & 0 & 0 & 0 & 0 & 0 & 0 & 0 & 0 \\
\hline Creatinine & 0 & 0 & 0 & 0 & 0 & 0 & 0 & 0 & 0 & 0 & 0 & 0 & 0 & 0 & 0 & 0 & 0 \\
\hline Ethanolamine & 1 & 1 & 1 & 1 & 1 & 1 & 0 & 1 & 1 & 0 & 0 & 1 & 0 & 1 & 1 & 1 & 1 \\
\hline Ethylamine & 0 & 0 & 0 & 0 & 0 & 0 & 0 & 0 & 0 & 0 & 0 & 1 & 0 & 0 & 0 & 0 & 0 \\
\hline Urea & 0 & 0 & 0 & 0 & 0 & 1 & 0 & 0 & 0 & 0 & 0 & 0 & 0 & 0 & 0 & 1 & 0 \\
\hline Hexylamine & 0 & 0 & 0 & 0 & 0 & 0 & 0 & 0 & 0 & 0 & 0 & 0 & 0 & 0 & 0 & 0 & 0 \\
\hline Putrescine & 1 & 1 & 1 & 1 & 1 & 1 & 1 & 1 & 0 & 1 & 1 & 1 & 1 & 1 & 1 & 1 & 1 \\
\hline Spermidine & 1 & 1 & 1 & 1 & 1 & 1 & 0 & 1 & 0 & 1 & 1 & 1 & 1 & l & 1 & 1 & 1 \\
\hline Spermine & 0 & 0 & 0 & 0 & 0 & 0 & 0 & 0 & 0 & 0 & 0 & 0 & 0 & 0 & 0 & 0 & 1 \\
\hline Taurine & 0 & 0 & 0 & 0 & 0 & 0 & 0 & 0 & 0 & 0 & 0 & 0 & 0 & 0 & 0 & 0 & 0 \\
\hline Trigonelline & 0 & 0 & 1 & 0 & 0 & 0 & 0 & 0 & 0 & 0 & 1 & 0 & 0 & 0 & 0 & 1 & 0 \\
\hline Tryptamine & 0 & 0 & 0 & 0 & 0 & 0 & 0 & 0 & 0 & 0 & 0 & 0 & 0 & 0 & 0 & 0 & 0 \\
\hline Tyramine & 1 & 1 & 1 & 1 & 1 & 0 & 0 & 0 & 0 & 0 & 1 & 0 & 0 & 0 & 1 & 1 & 0 \\
\hline \multicolumn{18}{|l|}{ Miscellaneous nitrogenous compounds } \\
\hline Acetamide & 0 & 0 & 0 & 0 & 0 & 0 & 1 & 0 & 1 & 0 & 0 & 0 & 0 & 0 & 1 & 0 & 0 \\
\hline Allantoin & 0 & 0 & 0 & 0 & 0 & 0 & 0 & 0 & 0 & 0 & 1 & 0 & 0 & 0 & 1 & 1 & 0 \\
\hline Cytosine & 0 & 0 & 0 & 0 & 0 & 1 & 0 & 1 & 0 & 0 & 0 & 0 & 0 & 0 & 0 & 0 & 0 \\
\hline Glycinamide & 0 & 0 & 0 & 0 & 0 & 1 & 1 & 1 & 1 & 0 & 0 & 0 & 0 & 0 & 0 & 1 & 0 \\
\hline Hippurate & 0 & 0 & 0 & 0 & 0 & 0 & 0 & 0 & 0 & 0 & 1 & 0 & 1 & 0 & 0 & 0 & 0 \\
\hline Indole-3-acetate & 0 & 1 & 0 & 0 & 0 & 0 & 0 & 0 & 0 & 0 & 0 & 0 & 0 & 0 & 0 & 0 & 0 \\
\hline L-Leucinamide & 0 & 1 & 0 & 0 & 1 & 0 & 1 & 1 & 0 & 1 & 1 & 1 & 0 & 0 & 1 & 1 & 1 \\
\hline Oxamic acid & 0 & 0 & 0 & 0 & 0 & 0 & 0 & 0 & 0 & 0 & 0 & 0 & 0 & 0 & 0 & 0 & 0 \\
\hline Salicylamide & 0 & 0 & 0 & 0 & 0 & 0 & 0 & 0 & 0 & 0 & 0 & 0 & 0 & 0 & 0 & 0 & 0 \\
\hline Thiamin & 0 & 0 & 0 & 0 & 0 & 0 & 0 & 0 & 0 & 0 & 0 & 0 & 0 & 0 & 0 & 0 & 0 \\
\hline Uracil & 0 & 0 & 0 & 0 & 0 & 0 & 0 & 1 & 0 & 0 & 0 & 0 & 0 & 0 & 0 & 0 & 1 \\
\hline Ureidosuccinate & 0 & 0 & 0 & 0 & 0 & 0 & 0 & 0 & 0 & 0 & 0 & 0 & 0 & 0 & 0 & 0 & 0 \\
\hline L-Valinamide & 1 & 1 & 1 & 1 & 1 & 1 & 0 & 1 & 1 & 0 & 0 & 1 & 0 & 0 & 0 & 1 & 1 \\
\hline \multicolumn{18}{|l|}{ Aromatic compounds } \\
\hline Anthranilate & 0 & 0 & 1 & 0 & 0 & 0 & 1 & 1 & 0 & 0 & 0 & 0 & 0 & 1 & 1 & 0 & 0 \\
\hline 3-Aminobenzoate & 0 & 0 & 1 & 0 & 0 & 0 & 0 & 0 & 0 & 0 & 1 & 0 & 0 & 0 & 0 & 0 & 0 \\
\hline 4-Aminobenzoate & 0 & 0 & 0 & 0 & 0 & 0 & 0 & 0 & 0 & 0 & 0 & 0 & 0 & 0 & 0 & 0 & 0 \\
\hline Benzoate & 0 & 0 & 0 & 0 & 0 & 0 & 0 & 0 & 0 & 1 & 1 & 0 & 0 & 0 & 0 & 0 & 1 \\
\hline Furan-2-carbonic acid & 0 & 0 & 0 & 0 & 0 & 0 & 0 & 0 & 0 & 0 & 0 & 0 & 0 & 0 & 0 & 0 & 0 \\
\hline 3-Hydroxybenzoate & 0 & 0 & 1 & 0 & 0 & 0 & 0 & 0 & 0 & 1 & 1 & 0 & 0 & 0 & 0 & 0 & 0 \\
\hline 4-Hydroxybenzoate & 0 & 0 & 1 & 0 & 0 & 1 & 0 & 0 & 1 & 1 & 0 & 0 & 1 & l & 1 & 0 & 1 \\
\hline 2-Hydroxyphenylacetate & 0 & 0 & 0 & 0 & 0 & 0 & 0 & 0 & 0 & 0 & 0 & 0 & 0 & 0 & 1 & 0 & 0 \\
\hline 3-Hydroxyphenylacetate & 0 & 0 & 0 & 0 & 1 & 0 & 0 & 1 & 1 & 0 & 0 & 0 & 0 & 1 & 1 & 0 & 0 \\
\hline 4-Hydroxyphenylacetate & 1 & 0 & 1 & 0 & 0 & 0 & 1 & 0 & 0 & 0 & 0 & 0 & 0 & 1 & 1 & 0 & 0 \\
\hline Isophthalate & 0 & 0 & 0 & 0 & 0 & 0 & 0 & 0 & 0 & 0 & 0 & 0 & 0 & 0 & 0 & 0 & 0 \\
\hline D-Mandelate & 0 & 0 & 0 & 0 & 0 & 0 & 0 & 0 & 0 & 0 & 0 & 0 & 0 & 0 & 0 & 0 & 0 \\
\hline L-Mandelate & 0 & 0 & 0 & 0 & 0 & 0 & 0 & 0 & 0 & 0 & 0 & 0 & 0 & 0 & 0 & 0 & 0 \\
\hline Phenoxyacetate & 0 & 0 & 0 & 0 & 0 & 0 & 0 & 0 & 0 & 0 & 0 & 0 & 0 & 0 & 0 & 0 & 0 \\
\hline Phenylacetate & 1 & 1 & 0 & 1 & 0 & 0 & 1 & 1 & 1 & 0 & 1 & 1 & 1 & 1 & 1 & 1 & 1 \\
\hline Phenyllactate & 1 & 0 & 1 & 1 & 1 & 1 & 0 & 1 & 0 & 1 & 0 & 0 & 1 & 1 & 0 & 1 & 1 \\
\hline Phenylpropionate & 0 & 0 & 0 & 1 & 0 & 0 & 0 & 0 & 0 & 1 & 1 & 0 & 0 & 0 & 0 & 0 & 0 \\
\hline Phenylpyruvate & 0 & 0 & 0 & 0 & 1 & 1 & 0 & 1 & 1 & 0 & 1 & 1 & 1 & 1 & 1 & 0 & 0 \\
\hline Phthalate & 0 & 0 & 0 & 0 & 0 & 0 & 0 & 0 & 0 & 0 & 0 & 0 & 0 & 0 & 0 & 0 & 0 \\
\hline Protocatechuate & 0 & 0 & 0 & 1 & 1 & 0 & 0 & 1 & 0 & 0 & 1 & 0 & 0 & 1 & 0 & 0 & 0 \\
\hline Quinate & 0 & 0 & 1 & 0 & 0 & 0 & 0 & 0 & 0 & 0 & 1 & 0 & 1 & 1 & 1 & 0 & 1 \\
\hline Salicylate & 0 & 0 & 0 & 0 & 0 & 0 & 0 & 0 & 0 & 0 & 1 & 0 & 0 & 1 & 1 & 0 & 0 \\
\hline Tropate & 0 & 0 & 0 & 0 & 0 & 0 & 0 & 1 & 0 & 0 & 0 & 0 & 0 & 0 & 0 & 0 & 0 \\
\hline Vanillate & 0 & 0 & 1 & 0 & 0 & 0 & 0 & 0 & 0 & 0 & 0 & 0 & 0 & 0 & 1 & 0 & 0 \\
\hline \multicolumn{18}{|l|}{ Growth with: } \\
\hline Glucose $+1 \% \mathrm{NaCl}$ & 1 & 1 & 1 & 1 & 1 & 1 & 1 & 1 & 1 & 1 & 1 & 1 & 1 & 1 & 1 & 1 & 1 \\
\hline Glucose $+2 \% \mathrm{NaCl}$ & 1 & 1 & 1 & 1 & 1 & 1 & 1 & 1 & 1 & 1 & 1 & 1 & 1 & 1 & 1 & 1 & 1 \\
\hline Glucose $+5 \% \mathrm{NaCl}$ & 0 & 1 & 0 & 0 & 0 & 1 & 1 & 1 & 1 & 1 & 1 & 1 & 1 & 1 & 1 & 1 & 1 \\
\hline Glucose $+7 \% \mathrm{NaCl}$ & 0 & 1 & 0 & 0 & 0 & 1 & 1 & 1 & 1 & 1 & 1 & 1 & 1 & 1 & 1 & 1 & 1 \\
\hline Glucose $+10 \% \mathrm{NaCl}$ & 0 & 1 & 0 & 1 & 0 & 1 & 1 & 1 & 1 & 1 & 1 & 1 & 1 & 1 & 1 & 0 & 1 \\
\hline Glucose $+0.005 \% \mathrm{NaN}_{3}$ & 1 & 1 & 1 & 0 & 1 & 1 & 0 & 1 & 1 & 1 & 1 & 0 & 0 & 0 & 1 & 0 & 1 \\
\hline Glucose $+0.01 \% \mathrm{NaN}_{3}$ & 1 & 1 & 1 & 0 & 1 & 1 & 0 & 1 & 1 & 1 & 1 & 0 & 0 & 0 & 1 & 0 & 1 \\
\hline Glucose $+0.02 \% \mathrm{NaN}_{3}$ & 1 & 0 & 0 & 0 & 1 & 0 & 0 & 0 & 0 & 1 & 1 & 1 & 0 & 0 & 0 & 0 & 0 \\
\hline
\end{tabular}




$\begin{array}{lllllllllllllllllllllllllllll}1 & 1 & 1 & 0 & 1 & 1 & 1 & 1 & 0 & 1 & 1 & 1 & 1 & 0 & 1 & 1 & 0 & 0 & 0 & 1 & 1 & 0 & 1 \\ 1 & 1 & 0 & 1 & 0 & 1 & 1 & 1 & 1 & 1 & 1 & 1 & 1 & 1 & 1 & 1 & 0 & 1 & 0 & 1 & 1 & 0 & 1\end{array}$

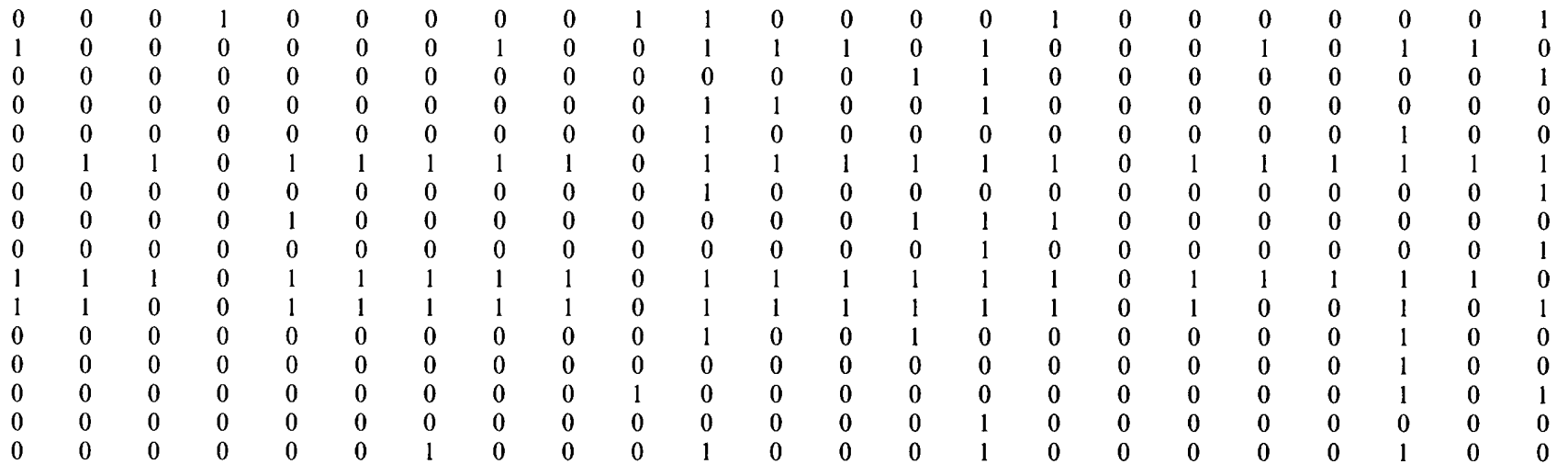

$\begin{array}{llllllllllllllllllllllllllllll}0 & 0 & 0 & 0 & 1 & 0 & 0 & 0 & 0 & 0 & 1 & 0 & 0 & 0 & 1 & 0 & 1 & 0 & 0 & 0 & 1 & 0 & 1 \\ 0 & 0 & 0 & 0 & 0 & 0 & 1 & 0 & 1 & 0 & 1 & 0 & 0 & 0 & 1 & 0 & 0 & 0 & 0 & 0 & 0 & 0 & 0 \\ 0 & 0 & 0 & 0 & 0 & 0 & 0 & 0 & 0 & 1 & 0 & 1 & 0 & 0 & 0 & 0 & 1 & 0 & 0 & 0 & 0 & 0 & 0 \\ 0 & 1 & 0 & 0 & 1 & 0 & 1 & 1 & 1 & 1 & 0 & 0 & 1 & 1 & 1 & 0 & 0 & 0 & 1 & 0 & 1 & 0 & 0 \\ 0 & 0 & 1 & 0 & 0 & 0 & 0 & 1 & 0 & 1 & 1 & 1 & 1 & 0 & 1 & 1 & 0 & 0 & 0 & 0 & 0 & 0 & 1 \\ 0 & 0 & 0 & 0 & 0 & 0 & 0 & 0 & 0 & 0 & 0 & 0 & 0 & 0 & 0 & 0 & 0 & 0 & 0 & 0 & 0 & 0 & 1 \\ 0 & 1 & 1 & 0 & 0 & 0 & 0 & 1 & 1 & 1 & 1 & 1 & 1 & 1 & 0 & 0 & 0 & 0 & 1 & 0 & 0 & 1 & 1 \\ 0 & 0 & 1 & 0 & 1 & 0 & 0 & 0 & 0 & 0 & 0 & 0 & 0 & 1 & 0 & 0 & 0 & 0 & 0 & 0 & 1 & 0 & 0 \\ 0 & 0 & 0 & 0 & 0 & 0 & 0 & 0 & 1 & 0 & 0 & 0 & 0 & 0 & 0 & 0 & 0 & 0 & 0 & 0 & 1 & 0 & 0 \\ 0 & 0 & 0 & 0 & 0 & 0 & 0 & 0 & 0 & 0 & 0 & 0 & 1 & 0 & 0 & 0 & 1 & 0 & 0 & 0 & 0 & 0 & 0 \\ 0 & 0 & 0 & 0 & 0 & 0 & 0 & 0 & 0 & 1 & 0 & 1 & 0 & 1 & 0 & 0 & 0 & 0 & 0 & 0 & 0 & 0 & 1 \\ 0 & 0 & 0 & 0 & 0 & 0 & 0 & 0 & 0 & 0 & 0 & 0 & 0 & 0 & 0 & 0 & 0 & 0 & 0 & 0 & 0 & 0 & 0 \\ 0 & 1 & 1 & 1 & 0 & 0 & 1 & 1 & 1 & 1 & 1 & 1 & 1 & 0 & 0 & 1 & 0 & 0 & 0 & 0 & 0 & 0 & 1\end{array}$

\begin{tabular}{|c|c|c|c|c|c|c|c|c|c|c|c|c|c|c|c|c|c|c|c|c|c|}
\hline 0 & 0 & 0 & 0 & 1 & 1 & 0 & 0 & 0 & 0 & 1 & 1 & 1 & 0 & 1 & 0 & 0 & 1 & 0 & 0 & 1 & 0 \\
\hline 0 & 0 & 0 & 0 & 0 & 0 & 0 & 0 & 0 & 0 & 0 & 0 & 1 & 0 & 0 & 0 & 0 & 1 & 0 & 0 & 1 & 0 \\
\hline 0 & 0 & 0 & 0 & 0 & 0 & 0 & 0 & 0 & 0 & 0 & 0 & 0 & 0 & 0 & 0 & 0 & 0 & 0 & 0 & 1 & 0 \\
\hline 0 & 0 & 0 & 0 & 0 & 0 & 0 & 0 & 0 & 0 & 0 & 0 & 1 & 0 & 0 & 0 & 0 & 0 & 0 & 0 & 0 & 0 \\
\hline 0 & 0 & 0 & 0 & 0 & 0 & 0 & 0 & 0 & 1 & 0 & 1 & 0 & 0 & 0 & 1 & 0 & 0 & 0 & 0 & 0 & 0 \\
\hline 0 & 0 & 1 & 0 & 0 & 0 & 0 & 0 & 0 & 0 & 1 & 0 & 1 & 0 & 1 & 1 & 1 & 1 & 0 & 0 & 1 & 0 \\
\hline 0 & 0 & 0 & 0 & 1 & 1 & 0 & 0 & 1 & 0 & 1 & 1 & 1 & 0 & 1 & 1 & 1 & 0 & 0 & 0 & 1 & 0 \\
\hline 0 & 0 & 0 & $\dot{u}$ & 1 & 0 & 1 & 0 & 0 & 0 & 0 & 1 & 1 & 0 & 0 & 0 & 1 & 0 & 0 & 0 & 1 & 0 \\
\hline 0 & 0 & 0 & 0 & 1 & 1 & 1 & 0 & 0 & 0 & 0 & 0 & 1 & 0 & 1 & 0 & 1 & 0 & 0 & 0 & 0 & 0 \\
\hline 0 & 0 & 0 & 0 & 0 & 0 & 1 & 0 & 1 & 1 & 1 & 0 & 0 & 0 & 1 & 0 & 0 & 0 & 0 & 0 & 0 & 0 \\
\hline 0 & 0 & 0 & 1 & 0 & 0 & 0 & 0 & 0 & 0 & 0 & 0 & 0 & 0 & 0 & 0 & 0 & 0 & 0 & 0 & 0 & 0 \\
\hline 0 & 0 & 0 & 0 & 0 & 0 & 0 & 0 & 0 & 1 & 0 & 0 & 0 & 0 & 0 & 0 & 0 & 0 & 0 & 0 & 1 & 0 \\
\hline 0 & 0 & 0 & 0 & 0 & 0 & 0 & 0 & 0 & 0 & 0 & 0 & 0 & 0 & 0 & 0 & 0 & 0 & 0 & 0 & 0 & 0 \\
\hline 0 & 0 & 0 & 0 & 0 & 0 & 0 & 0 & 0 & 0 & 0 & 0 & 0 & 0 & 0 & 0 & 0 & 0 & 0 & 0 & 0 & 0 \\
\hline 0 & 0 & 1 & 0 & 0 & 1 & 1 & 0 & 1 & 1 & 1 & 0 & 1 & 0 & 1 & 0 & 0 & 0 & 0 & 0 & 1 & 1 \\
\hline 0 & 1 & 1 & 0 & 1 & 0 & 1 & 1 & 1 & 1 & 1 & 1 & 1 & 0 & 1 & 1 & 0 & 0 & 0 & 0 & 0 & 1 \\
\hline 0 & 0 & 0 & 0 & 0 & 0 & 0 & 0 & 0 & 0 & 0 & 0 & 0 & 0 & 0 & 0 & 1 & 0 & 0 & 0 & 0 & 0 \\
\hline 1 & 0 & 0 & 0 & 0 & 1 & 0 & 1 & 1 & 0 & 1 & 0 & 1 & 0 & 1 & 0 & 0 & 1 & 0 & 0 & 1 & 0 \\
\hline 0 & 0 & 0 & 0 & 0 & 0 & 0 & 0 & 0 & 0 & 0 & 0 & 0 & 0 & 0 & 0 & 0 & 0 & 0 & 0 & 0 & 0 \\
\hline 0 & 0 & 1 & 0 & 0 & 1 & 1 & 0 & 0 & 0 & 1 & 1 & 1 & 0 & 1 & 0 & 1 & 0 & 0 & 0 & 0 & 0 \\
\hline 0 & 0 & 0 & 0 & 1 & 0 & 0 & 0 & 0 & 0 & 1 & 0 & 1 & 1 & 1 & 1 & 0 & 0 & 0 & 0 & 0 & 0 \\
\hline 0 & 0 & 0 & 0 & 0 & 0 & 1 & 0 & 1 & 0 & 0 & 0 & 1 & 0 & 0 & 1 & 0 & 0 & 0 & 0 & 1 & 0 \\
\hline 0 & 0 & 0 & 0 & 0 & 0 & 0 & 0 & 0 & 0 & 0 & 0 & 0 & 0 & 0 & 1 & 0 & 0 & 0 & 0 & 0 & 1 \\
\hline 0 & 0 & 0 & 0 & 0 & 0 & 0 & 0 & 0 & 0 & 0 & 0 & 1 & 0 & 0 & 0 & 0 & 0 & 0 & 0 & 1 & 0 \\
\hline
\end{tabular}

$\begin{array}{lllllllllllllllllllllllllllllllllllll}1 & 1 & 1 & 1 & 1 & 1 & 1 & 1 & 1 & 1 & 1 & 1 & 1 & 1 & 1 & 1 & 1 & 1 & 0 & 1 & 1 & 0 & 1 \\ 1 & 1 & 1 & 1 & 1 & 1 & 1 & 1 & 1 & 1 & 1 & 1 & 1 & 1 & 1 & 1 & 1 & 0 & 0 & 0 & 1 & 1 & 1 \\ 1 & 1 & 0 & 1 & 0 & 1 & 1 & 0 & 0 & 1 & 1 & 1 & 1 & 1 & 1 & 1 & 1 & 0 & 0 & 0 & 1 & 1 & 1 \\ 0 & 0 & 0 & 0 & 0 & 1 & 1 & 0 & 0 & 1 & 1 & 1 & 1 & 1 & 1 & 1 & 0 & 0 & 0 & 0 & 0 & 1 & 1 \\ 0 & 0 & 0 & 0 & 0 & 1 & 1 & 0 & 0 & 1 & 1 & 0 & 1 & 1 & 1 & 1 & 0 & 0 & 0 & 0 & 0 & 0 & 1 \\ 0 & 0 & 0 & 1 & 1 & 1 & 1 & 0 & 0 & 0 & 1 & 1 & 0 & 1 & 1 & 1 & 0 & 0 & 0 & 0 & 0 & 0 & 0 \\ 0 & 0 & 0 & 1 & 0 & 1 & 1 & 0 & 0 & 0 & 1 & 1 & 0 & 1 & 0 & 1 & 0 & 0 & 0 & 0 & 0 & 0 & 0 \\ 0 & 0 & 0 & 1 & 0 & 0 & 0 & 0 & 0 & 0 & 1 & 1 & 0 & 1 & 0 & 0 & 0 & 0 & 0 & 0 & 0 & 0 & 0\end{array}$


Table 4 (continued)

\begin{tabular}{llllllllllllllllllllll}
\hline \hline & Single-member \\
Character & cluster number $\ldots$ & $1-8$ & 15 & 16 & 18 & 19 & 29 & 32 & 34 & 37 & 38 & 39 & 40 & 42 & 43 & 45 & 46 & 47 & \\
\hline
\end{tabular}

Hydrolysis of:

Glycosidase substrates

pNP- $N$-acetyl- $\beta$-D-galactosaminide

pNP- $N$-acetyl- $\alpha$-D-glucosaminide

pNP- $N$-acetyl- $\beta$-D-glucosaminide

pNP- $\alpha$-L-arabinopyranoside

pNP- $\beta$-D-cellobioside

pNP- $\alpha$-L-fucopyranoside

pNP- $\beta$-L-fucopyranoside

pNP- $\beta$-D-galactopyranoside

pNP- $\beta$-D-galacturonide

pNP- $\alpha$-D-glucopyranoside

pNP- $\beta$-D-glucopyranoside

pNP- $\beta$-D-glucuronide

pNP- $\beta$-D-lactoside

pNP- $\alpha$-D-mannopyranoside

pNP- $\alpha$-D-maltoside

pNP- $\beta$-D-maltoside

pNP- $\beta$-D-melibioside

pNP- $\alpha$-L-rhamnopyranoside

pNP- $\beta$-D-xyloside

Sulphatase and phosphatase substrates

pNP-sulphate

pNP-phosphate (pH 7.0)

pNP-phosphate (pH 5.4)

pNP-phosphate (pH 8.2)

bis-pNP-phosphate

pNP-phenylphosphonate

pNP-phosphorylcholine

2-Desoxythymidine-5'-pNP-phosphate

Aminopeptidase substrates

Acetyl-L-alanine-pNA

Acetyl-L-leucine-pNA

D-Alanine-pNA

L-Alanine-pNA

L-Arginine-pNA

Benzoyl-DL-arginine-pNA

Glutamate-1-pNA

$\gamma$-L-Glutamate-pNA

L-Glutamate- $\gamma$-3-carboxy-pNA

Glycine-pNA

L-Lysine-pNA

L-Phenylalanine-pNA

L-Proline-pNA

Succinyl-L-phenylalanine-pNA

L-Valine-pNA

Acid formation from:

Carbohydrates and sugar derivatives

$N$-Acetyl-D-Glucosamine

D-Arabinose

L-Arabinose

Arabonic acid

D-Cellobiose

Dextran

D-Fructose

D-Galactose

$\alpha$-D-Galacturonate

Gluconate

D-Glucosamine

D-Glucose

D-Glucuronate

Glycogen

Inulin

Lactose

Lactobionic acid

Lactulose

$\begin{array}{lllllllllllllllll}1 & 1 & 1 & 0 & 1 & 1 & 1 & 1 & 1 & 1 & 1 & 1 & 0 & 1 & 1 & 1 & 1 \\ 0 & 0 & 0 & 0 & 0 & 1 & 1 & 0 & 1 & 1 & 0 & 1 & 0 & 1 & 0 & 0 & 0 \\ 1 & 1 & 1 & 1 & 1 & 1 & 1 & 1 & 1 & 1 & 1 & 1 & 1 & 1 & 1 & 1 & 1 \\ 1 & 1 & 1 & 1 & 1 & 1 & 1 & 0 & 0 & 0 & 0 & 0 & 1 & 1 & 1 & 1 & 0 \\ 1 & 1 & 1 & 1 & 1 & 1 & 1 & 1 & 0 & 1 & 0 & 1 & 1 & 1 & 1 & 1 & 1 \\ 0 & 0 & 0 & 0 & 1 & 0 & 0 & 1 & 0 & 0 & 1 & 0 & 1 & 0 & 0 & 0 & 0 \\ 0 & 0 & 1 & 1 & 0 & 1 & 0 & 0 & 1 & 0 & 0 & 1 & 1 & 1 & 1 & 1 & 1 \\ 1 & 1 & 1 & 1 & 1 & 1 & 1 & 1 & 1 & 0 & 0 & 1 & 0 & 1 & 1 & 0 & 1 \\ 0 & 0 & 1 & 0 & 0 & 0 & 0 & 0 & 0 & 0 & 0 & 0 & 0 & 0 & 0 & 0 & 0 \\ 1 & 1 & 1 & 1 & 1 & 1 & 1 & 1 & 1 & 1 & 1 & 1 & 1 & 1 & 1 & 1 & 1 \\ 1 & 1 & 1 & 1 & 1 & 1 & 1 & 1 & 1 & 1 & 1 & 1 & 1 & 1 & 1 & 1 & 1 \\ 0 & 0 & 0 & 0 & 0 & 0 & 0 & 0 & 0 & 0 & 0 & 0 & 0 & 0 & 0 & 0 & 0 \\ 1 & 1 & 1 & 1 & 0 & 1 & 1 & 0 & 0 & 0 & 0 & 0 & 0 & 1 & 1 & 1 & 1 \\ 1 & 1 & 1 & 1 & 1 & 1 & 1 & 1 & 1 & 1 & 1 & 1 & 1 & 1 & 1 & 1 & 0 \\ 1 & 1 & 1 & 1 & 1 & 1 & 0 & 1 & 0 & 0 & 1 & 1 & 0 & 1 & 1 & 1 & 1 \\ 1 & 1 & 1 & 0 & 1 & 1 & 1 & 1 & 0 & 0 & 1 & 0 & 1 & 1 & 1 & 1 & 1 \\ 0 & 1 & 1 & 0 & 0 & 0 & 1 & 0 & 0 & 0 & 0 & 0 & 0 & 1 & 1 & 1 & 0 \\ 0 & 0 & 1 & 1 & 1 & 0 & 0 & 0 & 1 & 1 & 0 & 1 & 0 & 1 & 1 & 0 & 1 \\ 1 & 1 & 1 & 1 & 1 & 1 & 1 & 0 & 1 & 1 & 1 & 1 & 1 & 1 & 1 & 1 & 0 \\ & & & & & & & & & & & & & & & & \\ 0 & 0 & 0 & 0 & 0 & 0 & 0 & 0 & 0 & 0 & 0 & 0 & 0 & 1 & 0 & 0 & 0 \\ 1 & 1 & 1 & 1 & 1 & 1 & 1 & 1 & 1 & 1 & 1 & 1 & 1 & 1 & 1 & 1 & 1 \\ 1 & 1 & 1 & 1 & 1 & 1 & 1 & 1 & 1 & 1 & 1 & 1 & 1 & 1 & 1 & 1 & 1 \\ 1 & 1 & 1 & 1 & 1 & 1 & 1 & 1 & 1 & 1 & 1 & 1 & 1 & 1 & 1 & 1 & 1 \\ 1 & 1 & 1 & 1 & 1 & 1 & 1 & 1 & 1 & 1 & 1 & 1 & 1 & 1 & 1 & 1 & 1 \\ 1 & 1 & 1 & 1 & 1 & 1 & 1 & 1 & 1 & 1 & 1 & 1 & 1 & 1 & 1 & 1 & 1 \\ 1 & 1 & 1 & 1 & 1 & 1 & 1 & 1 & 1 & 1 & 1 & 1 & 1 & 1 & 1 & 1 & 1 \\ 1 & 1 & 1 & 1 & 1 & 1 & 1 & 1 & 1 & 1 & 1 & 1 & 1 & 1 & 1 & 1 & 1\end{array}$

$\begin{array}{lllll}0 & 1 & 1 & 0 & 0 \\ 0 & 0 & 0 & 0 & 0 \\ 0 & 1 & 1 & 0 & 0 \\ 0 & 0 & 0 & 0 & 0 \\ 0 & 0 & 0 & 0 & 0 \\ 0 & 0 & 0 & 0 & 0 \\ 0 & 1 & 1 & 0 & 0 \\ 0 & 0 & 0 & 0 & 0 \\ 0 & 1 & 0 & 0 & 1 \\ 0 & 1 & 0 & 0 & 0 \\ 0 & 1 & 1 & 1 & 1 \\ 0 & 0 & 0 & 0 & 0 \\ 0 & 0 & 1 & 0 & 1 \\ 0 & 0 & 0 & 0 & 0 \\ 0 & 1 & 0 & 0 & 0 \\ 0 & 1 & 1 & 0 & 0 \\ 0 & 0 & 0 & 0 & 1 \\ 0 & 1 & 1 & 0 & 0\end{array}$

$\begin{array}{ll}1 & 1 \\ 0 & 1 \\ 0 & 0 \\ 0 & 0 \\ 1 & 0 \\ 0 & 0 \\ 0 & 0 \\ 1 & 0 \\ 0 & 0 \\ 1 & 1 \\ 1 & 0 \\ 1 & 0 \\ 0 & 0 \\ 0 & 0 \\ 0 & 1 \\ 0 & 0 \\ 0 & 0 \\ 0 & 0\end{array}$

$$
\begin{array}{lll}
1 & 0 & 0 \\
1 & 0 & 0 \\
1 & 0 & 0 \\
0 & 0 & 0 \\
1 & 0 & 0 \\
0 & 0 & 0 \\
1 & 0 & 0 \\
1 & 1 & 0 \\
0 & 0 & 1 \\
0 & 0 & 0 \\
1 & 1 & 1 \\
1 & 1 & 0 \\
0 & 0 & 0 \\
0 & 0 & 0 \\
0 & 0 & 0 \\
0 & 0 & 0 \\
0 & 0 & 1 \\
0 & 0 & 0
\end{array}
$$$$
\begin{array}{ll}
0 & 0 \\
0 & 0 \\
0 & 0 \\
0 & 0 \\
0 & 0 \\
0 & 0 \\
0 & 0 \\
0 & 0 \\
0 & 0 \\
0 & 0 \\
0 & 1 \\
0 & 0 \\
0 & 0 \\
0 & 0 \\
0 & 0 \\
0 & 0 \\
0 & 0 \\
0 & 0
\end{array}
$$ 


\begin{tabular}{lllllllllllllllllllllllll}
48 & 52 & 56 & 58 & 59 & 60 & 61 & 62 & 63 & 67 & 70 & 71 & 72 & 73 & 74 & 75 & 77 & 81 & 83 & 84 & 85 & 86 & 87 \\
\hline
\end{tabular}

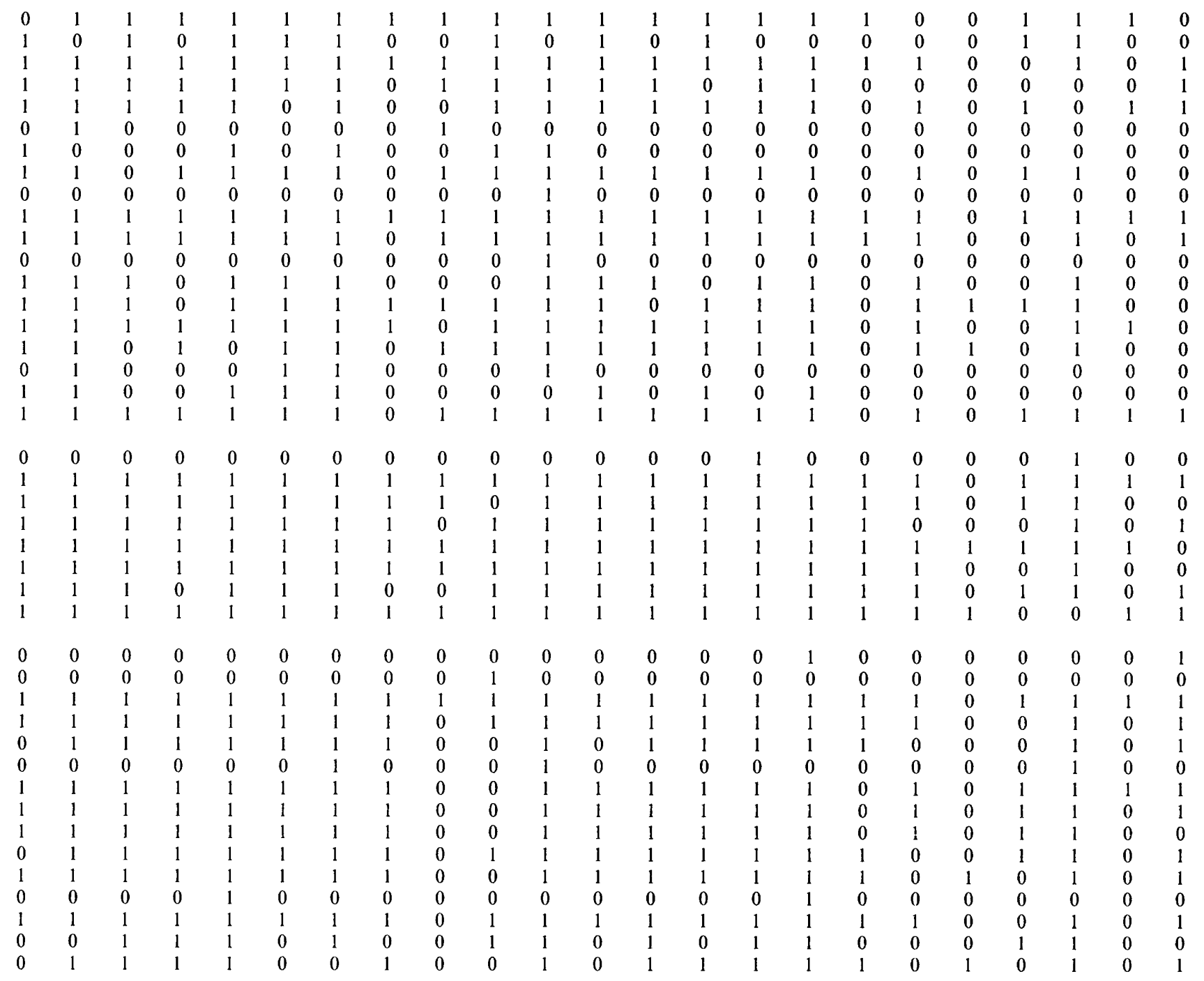

$\begin{array}{llllllllllllllllllllllll}1 & 0 & 0 & 0 & 0 & 1 & 0 & 0 & 0 & 0 & 1 & 0 & 0 & 0 & 1 & 0 & 0 & 0 & 1 & 1 & 1 & 0 & 1 \\ 0 & 0 & 0 & 0 & 0 & 0 & 1 & 0 & 0 & 0 & 0 & 0 & 0 & 1 & 0 & 0 & 0 & 0 & 0 & 0 & 0 & 0 & 0 \\ 1 & 0 & 1 & 0 & 0 & 0 & 1 & 0 & 0 & 0 & 0 & 0 & 0 & 0 & 1 & 1 & 0 & 0 & 0 & 0 & 1 & 0 & 1 \\ 0 & 0 & 0 & 0 & 0 & 0 & 0 & 0 & 0 & 0 & 0 & 0 & 0 & 0 & 0 & 0 & 0 & 0 & 0 & 0 & 0 & 0 & 0 \\ 1 & 0 & 0 & 0 & 0 & 0 & 0 & 0 & 0 & 0 & 0 & 0 & 0 & 0 & 1 & 1 & 0 & 0 & 0 & 0 & 1 & 0 & 1 \\ 0 & 0 & 0 & 0 & 0 & 0 & 0 & 0 & 0 & 0 & 0 & 0 & 0 & 0 & 0 & 0 & 0 & 0 & 0 & 0 & 0 & 0 & 0 \\ 0 & 0 & 0 & 0 & 1 & 1 & 0 & 0 & 0 & 0 & 1 & 0 & 1 & 0 & 1 & 1 & 0 & 0 & 1 & 0 & 1 & 0 & 1 \\ 0 & 0 & 0 & 0 & 0 & 0 & 1 & 0 & 0 & 0 & 0 & 0 & 1 & 1 & 1 & 0 & 0 & 0 & 0 & 0 & 1 & 0 & 1 \\ 0 & 1 & 0 & 0 & 0 & 0 & 0 & 1 & 0 & 0 & 0 & 1 & 0 & 1 & 0 & 0 & 1 & 0 & 0 & 0 & 0 & 0 & 1 \\ 0 & 0 & 0 & 0 & 0 & 0 & 0 & 1 & 0 & 0 & 0 & 0 & 0 & 0 & 0 & 0 & 1 & 0 & 0 & 0 & 1 & 0 & 0 \\ 1 & 1 & 0 & 0 & 1 & 1 & 1 & 1 & 0 & 1 & 0 & 1 & 1 & 0 & 0 & 1 & 1 & 1 & 1 & 1 & 1 & 0 & 1 \\ 1 & 0 & 0 & 0 & 0 & 0 & 1 & 0 & 0 & 0 & 0 & 0 & 0 & 1 & 1 & 1 & 0 & 0 & 0 & 0 & 1 & 0 & 1 \\ 1 & 0 & 0 & 0 & 0 & 0 & 0 & 0 & 0 & 0 & 0 & 1 & 0 & 0 & 0 & 1 & 0 & 0 & 0 & 0 & 0 & 0 & 0 \\ 0 & 0 & 0 & 0 & 0 & 0 & 1 & 0 & 0 & 0 & 0 & 0 & 0 & 0 & 1 & 1 & 0 & 1 & 0 & 0 & 1 & 0 & 0 \\ 0 & 0 & 0 & 0 & 0 & 0 & 0 & 0 & 0 & 0 & 0 & 0 & 0 & 0 & 0 & 0 & 0 & 0 & 0 & 0 & 0 & 0 & 0 \\ 0 & 0 & 0 & 0 & 0 & 1 & 1 & 0 & 0 & 0 & 0 & 0 & 1 & 0 & 0 & 0 & 0 & 0 & 0 & 0 & 0 & 0 & 0 \\ 0 & 0 & 0 & 0 & 0 & 0 & 0 & 0 & 0 & 0 & 0 & 1 & 0 & 0 & 0 & 0 & 0 & 0 & 0 & 0 & 0 & 0 & 0 \\ 0 & 0 & 0 & 0 & 0 & 0 & 0 & 0 & 0 & 0 & 0 & 0 & 0 & 0 & 0 & 0 & 0 & 0 & 0 & 0 & 0 & 0 & 0\end{array}$


Table 4 (continued)

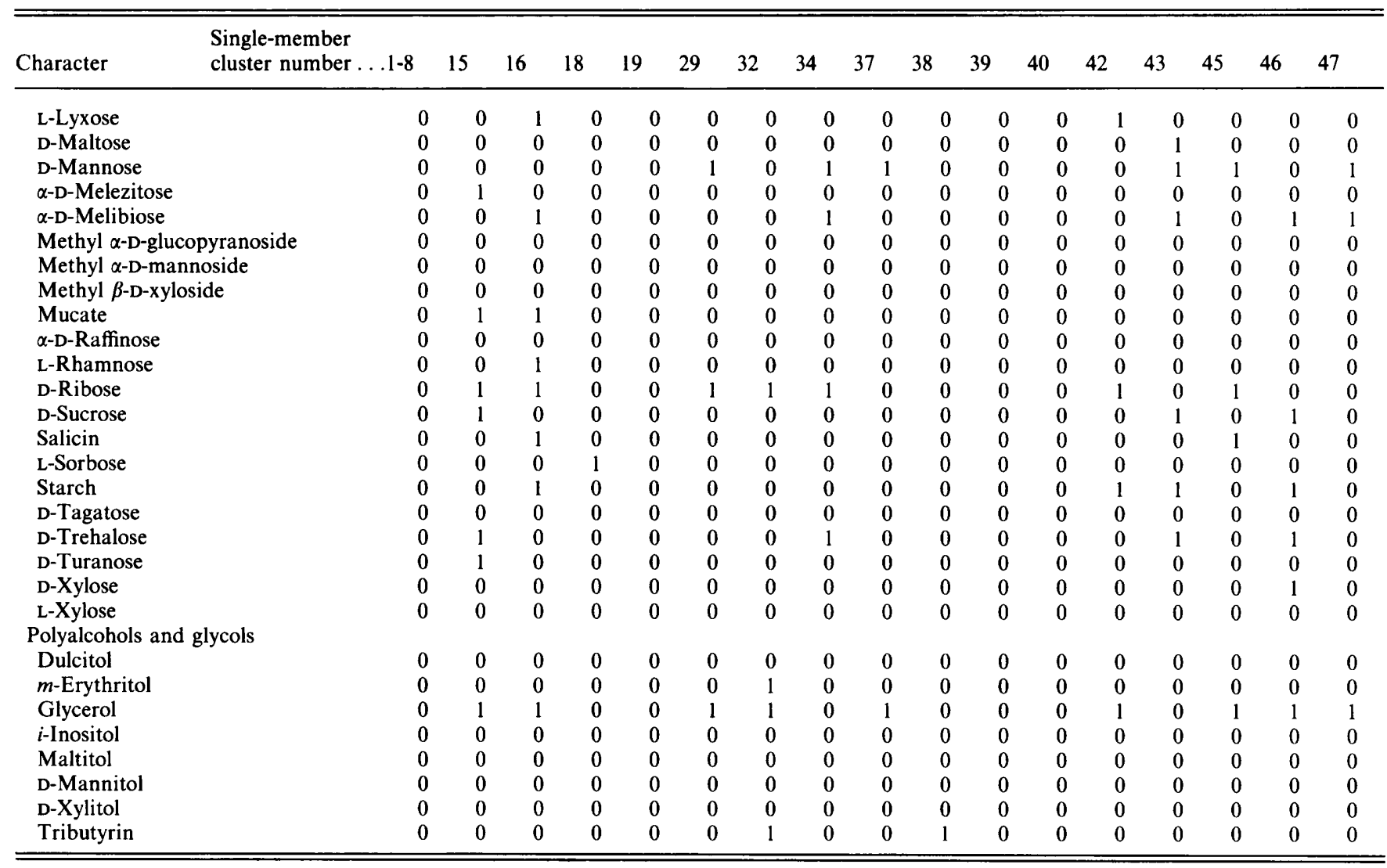

contained a further 105 of the 125 strains grouped into $S_{\mathrm{J}}$ subcluster 1-19, and four of the eight strains from $S_{\mathrm{J}}$ subcluster 1-9. One additional strain from $S_{\mathrm{J}}$ subcluster 1-19 was found in $S_{\mathrm{SM}}$ cluster 10 (S. sparsogenes), which also contained a strain of $S_{\mathrm{J}}$ subcluster 1-29; the remaining strains of $S_{\mathrm{J}}$ subcluster 1-19 were found in $S_{\mathrm{SM}}$ clusters 12 (S. aurantiacus) and 13 (S. niveoruber) and the single-member clusters 15,16 and 46 . Cluster 11 of the UPGMA $/ S_{\text {SM }}$ analysis ( $S$. psammoticus) contained four of the eight strains from $S_{\mathrm{J}}$ subcluster 1-20 and one strain from $S_{\mathrm{J}}$ subcluster 1-21; further strains of $S_{\mathrm{J}}$ subcluster 120 were found in $S_{\mathrm{SM}}$ cluster 20 (one strain) and, as mentioned above, in $S_{\mathrm{J}}$ subcluster 1-2 (three strains). The remaining strain of $S_{\mathrm{J}}$ subcluster 1-21 ( $S$. ghanaensis DSM 40746) was grouped into $S_{\mathrm{SM}}$ subcluster 1-7 ( $S$. diastatochromogenes). $S_{\mathrm{SM}}$ clusters 14 (Streptomyces $\mathrm{sp}$.) and 17 (S. griseoloalbus) were recovered as $S_{\mathrm{J}}$ clusters 21 and 7 , respectively. The two $S_{\mathrm{SM}}$ single-member clusters 18 (S. griseoruber) and 19 (S. cyaneogriseus) were joined to form $S_{\mathrm{J}}$ cluster 23 . Four strains of $S_{\mathrm{SM}}$ cluster $20(S$. chrysomallus) formed $S_{\mathrm{J}}$ cluster 32 ; one of the remaining two strains was grouped into $S_{\mathrm{J}}$ subcluster 1-8, which contained the remaining 10 strains of the $S_{\mathrm{SM}}$ subcluster
22-3 ( $S$. lavendulae), the other was assigned to $S_{\mathrm{J}}$ subcluster 1-20. The three thermophilic Streptomyces strains assigned to $S_{\mathrm{SM}}$ cluster 21 (S. thermovulgaris) were grouped into $S_{\mathrm{J}}$ cluster 2 , which also contained the two strains of cluster 68 ( $S$. sulphureus). The remaining two strains of $S_{\mathrm{SM}}$ cluster 21 were found in $S_{\mathrm{J}}$ subcluster 1-5, which contained two additional strains, from $S_{\mathrm{SM}}$ subclusters 22-3 ( $S$. lavendulae) and 22-5 ( $S$. fradiae), respectively.

The subclusters of $S_{\mathrm{SM}}$ cluster 22 (Streptoverticillium $\mathrm{sp} . / S$. lavendulae/S. fradiae) were largely recovered as clusters in the UPGMA $/ S_{\mathrm{J}}$ analysis. Subcluster 22-1 ( $S v$. griseocarneum) was found in its entirety as $S_{\mathrm{J}}$ cluster 40 . Four strains of subcluster 22-2 ( $S$. roseoviridis) formed $S_{\mathrm{J}}$ cluster 37; one further strain was grouped into $S_{\mathrm{J}}$ subcluster 1-15, which contained 18 strains, 15 of which were found in $S_{\mathrm{SM}}$ subcluster 1-7 ( $S$. diastatochromogenes). The remaining two strains of $S_{\mathrm{J}}$ subcluster 1-15 were found in $S_{\mathrm{SM}}$ subcluster 1-6 ( $S$. flaveolus) and in cluster 25 ( $S$. lydicus). Strains of $S_{\mathrm{SM}}$ subcluster 22-3 ( $S$. lavendulae) were grouped into $S_{\mathrm{J}}$ subclusters 1-5, 1-7, 1-9 (one strain) and 1-8 (nine strains) and into $S_{\mathrm{J}}$ clusters 42 (27 strains) and 43 (three strains). The $S_{\mathrm{J}}$ cluster 42 


\begin{tabular}{|c|c|c|c|c|c|c|c|c|c|c|c|c|c|c|c|c|c|c|c|c|c|c|}
\hline 48 & 52 & 56 & 58 & 59 & 60 & 61 & 62 & 63 & 67 & 70 & 71 & 72 & 73 & 74 & 75 & 77 & 81 & 83 & 84 & 85 & 86 & 87 \\
\hline 0 & 0 & 0 & 0 & 0 & 0 & 1 & 0 & 0 & 0 & 0 & 0 & 0 & 1 & 0 & 0 & 0 & 0 & 0 & 0 & 1 & 0 & 0 \\
\hline 0 & 0 & 0 & 0 & 0 & 0 & 0 & 0 & 0 & 0 & 0 & 0 & 1 & 1 & 1 & 0 & 0 & 0 & 0 & 0 & 0 & 0 & 0 \\
\hline 0 & 0 & 0 & 0 & 0 & 0 & 1 & 0 & 0 & 0 & 0 & 0 & 1 & 1 & 0 & 0 & 0 & 0 & 0 & 0 & 1 & 0 & 0 \\
\hline 0 & 0 & 0 & 0 & 0 & 0 & 0 & 0 & 0 & 0 & 0 & 0 & 0 & 0 & 0 & 0 & 0 & 0 & 0 & 0 & 0 & 0 & 1 \\
\hline 0 & 0 & 0 & 0 & 0 & 0 & 1 & 0 & 0 & 0 & 0 & 0 & 0 & 0 & 0 & 0 & 0 & 0 & 0 & 0 & 0 & 0 & 0 \\
\hline 0 & 0 & 0 & 0 & 0 & 0 & 0 & 0 & 0 & 0 & 0 & 0 & 0 & 0 & 0 & 0 & 0 & 0 & 0 & 0 & 0 & 0 & 0 \\
\hline 0 & 0 & 0 & 0 & 0 & 0 & 0 & 0 & 0 & 0 & 0 & 0 & 0 & 0 & 0 & 0 & 0 & 0 & 0 & 0 & 0 & 0 & 0 \\
\hline 0 & 0 & 0 & 0 & 0 & 0 & 0 & 0 & 0 & 0 & 0 & 0 & 0 & 0 & 0 & 0 & 0 & 0 & 0 & 0 & 0 & 0 & 0 \\
\hline 0 & 1 & 0 & 0 & 0 & 0 & 1 & 1 & 0 & 0 & 0 & 1 & 0 & 0 & 0 & 0 & 1 & 0 & 0 & 0 & 0 & 0 & 1 \\
\hline 0 & 0 & 0 & 0 & 0 & 0 & 0 & 0 & 0 & 0 & 0 & 0 & 0 & 0 & 0 & 0 & 0 & 0 & 0 & 0 & 0 & 0 & 0 \\
\hline 1 & 0 & 0 & 0 & 0 & 0 & 0 & 0 & 0 & 0 & 0 & 0 & 0 & 0 & 1 & 1 & 0 & 1 & 0 & 0 & 1 & 0 & 0 \\
\hline 1 & 0 & 0 & 0 & 0 & 1 & 0 & 0 & 0 & 0 & 0 & 0 & 1 & 1 & 1 & 1 & 0 & 0 & 0 & 1 & 1 & 0 & 1 \\
\hline 0 & 0 & 0 & 0 & 0 & 0 & 1 & 0 & 0 & 0 & 0 & 0 & 0 & 1 & 0 & 0 & 0 & 0 & 0 & 0 & 0 & 0 & 1 \\
\hline 1 & 0 & 0 & 0 & 0 & 0 & 1 & 0 & 0 & 0 & 1 & 0 & 0 & 0 & 0 & 0 & 0 & 0 & 0 & I & 0 & 0 & 1 \\
\hline 0 & 0 & 0 & 0 & 0 & 0 & 0 & 0 & 0 & 0 & 0 & 0 & 0 & 0 & 0 & 0 & 0 & 0 & 0 & 0 & 0 & 0 & 0 \\
\hline 0 & 0 & 0 & 0 & 0 & 1 & 1 & 0 & 0 & 0 & 0 & 0 & 1 & 0 & 1 & 1 & 0 & 1 & 0 & 0 & 1 & 0 & 0 \\
\hline 0 & 0 & 0 & 0 & 0 & 0 & 0 & 0 & 0 & 0 & 0 & 0 & 0 & 0 & 0 & 0 & 0 & 0 & 0 & 0 & 0 & 0 & 0 \\
\hline 0 & 0 & 0 & 0 & 0 & 1 & 1 & 0 & 0 & 1 & 0 & 0 & 1 & 0 & 0 & 0 & 0 & 0 & 0 & 0 & 1 & 0 & 1 \\
\hline 0 & 0 & 0 & 0 & 0 & 0 & 0 & 0 & 0 & 0 & 0 & 0 & 0 & 0 & 0 & 0 & 0 & 0 & 1 & 0 & 0 & 0 & 1 \\
\hline 0 & 0 & 0 & 0 & 0 & 0 & 0 & 0 & 0 & 0 & 0 & 0 & 0 & 0 & 0 & 1 & 0 & 0 & 0 & 0 & 1 & 0 & 1 \\
\hline 0 & 0 & 0 & 0 & 0 & 0 & 0 & 0 & 0 & 0 & 0 & 0 & 0 & 0 & 0 & 0 & 0 & 0 & 0 & 0 & 0 & 0 & 0 \\
\hline 0 & 0 & 0 & 0 & 0 & 0 & 0 & 0 & 0 & 0 & 0 & 0 & 0 & 0 & 0 & 0 & 0 & 0 & 0 & 0 & 0 & 0 & 1 \\
\hline 0 & 0 & 0 & 0 & 0 & 0 & 0 & 0 & 0 & 0 & 0 & 0. & 0 & 0 & 0 & 0 & 0 & 0 & 0 & 0 & 1 & 0 & 1 \\
\hline 1 & 0 & 0 & 0 & 0 & 1 & 1 & 0 & 0 & 0 & 1 & 0 & 0 & 0 & 0 & 1 & 0 & 0 & 0 & 0 & 1 & 0 & 1 \\
\hline 0 & 0 & 0 & 0 & 0 & 0 & 0 & 0 & 0 & 0 & 0 & 0 & 0 & 0 & 0 & 0 & 0 & 0 & 0 & 0 & 1 & 0 & 1 \\
\hline 0 & 0 & 0 & 0 & 0 & 0 & 0 & 0 & 0 & 0 & 0 & 0 & 0 & 0 & 0 & 0 & 0 & 0 & 0 & 0 & 0 & 0 & 1 \\
\hline 1 & 0 & 0 & 0 & 0 & 0 & 1 & 0 & 0 & 0 & 0 & 0 & 0 & 0 & 0 & 0 & 0 & 0 & 0 & 0 & 1 & 0 & 1 \\
\hline 0 & 0 & 0 & 0 & 0 & 0 & 0 & 0 & 0 & 0 & 0 & 0 & 0 & 0 & 0 & 0 & 0 & 0 & 0 & 0 & 0 & 0 & 1 \\
\hline 0 & 0 & 0 & 0 & 0 & 0 & 0 & 0 & 0 & 0 & 0 & 0 & 0 & 0 & 0 & 0 & 0 & 0 & 0 & 0 & 0 & 0 & 1 \\
\hline
\end{tabular}

Table 5. Comparison of clusters and subcultures of the UPGMA/S $S_{S M}$ analysis with corresponding phena of the study of Williams et al. (1983a)

\begin{tabular}{|c|c|c|c|c|c|}
\hline $\begin{array}{l}\text { Cluster/ } \\
\text { subcluster } \\
\text { no(s). }\end{array}$ & Phenon & $\begin{array}{c}\text { Corresponding } \\
\text { cluster(s) of } \\
\text { Williams } \text { et al. }(1983 a)\end{array}$ & $\begin{array}{l}\text { No. of } \\
\text { strains used in } \\
\text { both studies }\end{array}$ & $\begin{array}{c}\text { No. of } \\
\text { strains found in } \\
\text { corresponding phena }\end{array}$ & $\begin{array}{l}\text { Agreement } \\
(\%)\end{array}$ \\
\hline $1-1$ & S. albidoflavus & AlA & 15 & 15 & $100 \cdot 0$ \\
\hline $1-2$ & S. halstedii & $\mathrm{AlC}$ & 9 & 5 & $55 \cdot 5$ \\
\hline $1-3$ & $S$. griseus & AlB & 29 & 23 & $79 \cdot 3$ \\
\hline 2 & S. exfoliatus & $\mathrm{A} 5+\mathrm{A} 6$ & $15+9$ & $12+3$ & $62 \cdot 5$ \\
\hline 6 & S. rochei & $\mathrm{A} 12+\mathrm{A} 21$ & $22+9$ & $18+7$ & $80 \cdot 6$ \\
\hline 9 & S. violaceus & $\mathrm{A} 18+\mathrm{A} 19+\mathrm{A} 20$ & $36+14+4$ & $28+8+4$ & $74 \cdot 1$ \\
\hline $22-3$ & $S$. lavendulae & $F 61+F 62$ & $12+3$ & $11+3$ & $93 \cdot 3$ \\
\hline $22-5$ & $S$. fradiae & G68 & 2 & 2 & $100 \cdot 0$ \\
\hline 25 & S. lydicus & A29 & 11 & 7 & $63 \cdot 6$ \\
\hline $30,31,32$ & S. albus & A16 & 6 & 5 & $83 \cdot 3$ \\
\hline 33 & S. alboflavus & E54 & 3 & 3 & $100 \cdot 0$ \\
\hline 35,36 & S. rimosus & B42 & 6 & 4 & $66 \cdot 7$ \\
\hline $51,53-57$ & S. violaceusniger & A32 & 5 & 2 & $40 \cdot 0$ \\
\hline 69 & S. ochraceiscleroticus & A40 & 5 & 4 & $80 \cdot 0$ \\
\hline Total & & & 215 & 164 & $76 \cdot 3$ \\
\hline
\end{tabular}

contained 27 strains, almost exclusively found in clusters F61 and F62 of Williams et al. (1983a). A further 11 strains of $S_{\mathrm{J}}$ cluster 43 were grouped into $S_{\mathrm{SM}}$ subcluster 22-4 ( $S$. misakiensis), which contained one strain of $S_{\mathrm{J}}$ subcluster 1-6, and $S_{\mathrm{J}}$ clusters 35 and 62 (single-member cluster 'S. pyridomyceticus' ISP 5024), and two strains of $S_{\text {J }}$ subcluster 1-7. With the exception of two strains, one of them forming $S_{\mathrm{J}}$ single-member cluster $38(S$. clavuligerus DSM 40751), subcluster 22-5 ( $S$. fradiae) was recovered as $S_{\mathrm{J}}$ cluster 39 . 
The two strains of $S_{\mathrm{SM}}$ cluster 23 ( $S$. griseolus) and strain $S$. roseochromogenes ISP 5463 ( $S_{\mathrm{SM}}$ subcluster 1-3) formed $S_{\mathrm{J}}$ cluster 4 . $S_{\mathrm{SM}}$ cluster 24 ( $S$. fungicidicus) was split into the $S_{\mathrm{J}}$ clusters 56 and 57 (single-member cluster). The remaining strain was grouped into $S_{\mathrm{J}}$ cluster 5 , containing 13 strains, 12 of which were found in $S_{\mathrm{SM}}$ cluster 25 ( $S$. lydicus). This cluster contained a further three strains from $S_{\mathrm{J}}$ subcluster 1-9 and one strain each from $S_{\mathrm{J}}$ subclusters 1-15 and 1-23. $S_{\mathrm{SM}}$ cluster $26(S$. flavidovirens) was split into $S_{\mathrm{J}}$ single-member cluster 33 and $S_{\mathrm{J}}$ cluster 27 , which contained one further strain $\left(S_{\mathrm{SM}}\right.$ single-member cluster $32, S$. albus ISP $5313^{\mathrm{T}}$ ). $S_{\mathrm{SM}}$ cluster 27 ( $S$. fradiae) was recovered as $S_{\mathrm{J}}$ cluster $31 ; S_{\mathrm{SM}}$ cluster 28 ( $S$. prasinus) contained the two remaining strains of $S_{\mathrm{J}}$ cluster 9 not grouped into $S_{\mathrm{SM}}$ subcluster 1-5 ( $S$. antibioticus). $S_{\mathrm{SM}}$ clusters 30 (S. albus) and 31 (S. cacaoi) were joined as $S_{\mathrm{J}}$ subcluster 1-34 with the exception of $S$. antibioticus DSM 41568 , which formed $S_{\mathrm{J}}$ single-member cluster 46. The $S_{\mathrm{SM}}$ clusters 33 ( $S$. alboflavus), 34 ( $S$. fulvissimus) and 35 ( $S$. rimosus) were joined to form $S_{\mathrm{J}}$ subcluster 1-33, with the exception of two strains which formed $S_{\mathrm{J}}$ cluster $41 . S_{\mathrm{SM}}$ clusters 36 ( $S$. rimosus) and 49 ( $S$. roseiscleroticus) were recovered as $S_{\mathrm{J}}$ clusters 45 and 22 , respectively. The two strains of $S_{\mathrm{SM}}$ cluster 41 ( $S$. endus) were recovered as $S_{\mathrm{J}}$ cluster 12 , which contained in addition the strain forming $S_{\mathrm{SM}}$ single-member cluster 85. $S_{\mathrm{SM}}$ clusters 44 (Streptomyces $\mathrm{sp}$.) and single-member cluster 45 were joined in $S_{\mathrm{J}}$ subcluster 1-24. The two strains of $S_{\mathrm{SM}}$ cluster 50 (S. violatus) were grouped into $S_{\mathrm{J}}$ cluster 19, which contained further strains found in $S_{\mathrm{SM}}$ clusters 8,9 and 12 . $S_{\mathrm{SM}}$ clusters $51,53,54$ and 55 , all containing strains of $S$. violaceusniger, $S$. hygroscopicus and related organisms, were joined in $S_{\mathrm{J}}$ cluster 18 , with the exception of one strain classified within the genus Nocardia (' $N$. capreola'), which was grouped into $S_{\mathrm{J}}$ subcluster 1-30 together with the three strains of $S_{\mathrm{SM}}$ cluster 64 ( $S$. longissimus). $S_{\mathrm{SM}}$ cluster 57 ( $S$. hygroscopicus) was split into $S_{\mathrm{J}}$ single-member clusters 52 and 53 . The two strains of $S_{\mathrm{SM}}$ cluster 65 (Streptomyces sp.) were clustered into $S_{\mathrm{J}}$ subcluster 1-29 together with one strain from $S_{\mathrm{SM}}$ cluster 10. $S_{\mathrm{SM}}$ clusters 66 ( $S$. flocculus) and 67 (Nocardia $\mathrm{sp}$.) were joined into $S_{\mathrm{J}}$ cluster 34 . $S_{\mathrm{SM}}$ cluster 68 ( $S$. sulphureus) was grouped with three thermophilic strains from $S_{\mathrm{SM}}$ cluster 21 into $S_{\mathrm{J}}$ cluster 2 .

$S_{\mathrm{SM}}$ cluster 69 ( $S$. ochraceiscleroticus) and the singlemember clusters 70,72 and 73 were joined into $S_{\mathrm{J}}$ subcluster 1-26. $S_{\mathrm{SM}}$ single-member cluster 71 ( $S$. poonensis) fell within the large $S_{\mathrm{J}}$ subcluster 1-19. $S_{\mathrm{SM}}$ cluster 76 (Streptomyces sp./Nocardia sp.) was divided into the $S_{\mathrm{J}}$ clusters 69 (four strains), 71 (two strains), and the two single-member clusters 72 and 73. The $S_{\mathrm{SM}}$ clusters 78 (S. fragilis), 79 (S. lipmanii), 80 (Streptomyces $\mathrm{sp}$.) and 82 (Streptomyces sp.) were split into $S_{\mathrm{J}}$ singlemember clusters (Table 1).
The following single-member clusters of the UPGMA/ $S_{\mathrm{SM}}$ analysis were recovered also as $S_{\mathrm{J}}$ single-member clusters: $29,37-40,42,43,47,48,52,56,58-63,74,75$, $77,81,83,84,86$ and 87 (Table 1).

\section{Discussion}

The numerical taxonomic study of Williams et al. (1983a) was the guiding survey for a taxonomic framework of the genus Streptomyces. As pointed out by these authors, the phena defined by numerical taxonomic studies are polythetic: no single character is indispensable or sufficient for group membership (Williams et al., 1983a). Numerical taxonomic studies are thus in sharp contrast to traditional concepts in streptomycete taxonomy, which used a few subjectively chosen characters for the recognition of taxa (Gause $e t$ al., 1957; Pridham et al., 1958; Hütter, 1967; Pridham \& Tresner, 1974 $a, b$; Pridham, 1976).

Possible problems in numerical taxonomic studies can be minimized by the choice of suitable tests and statistics (Sneath \& Johnson, 1972; Austin \& Colwell, 1977; O'Brien \& Colwell, 1987). It is necessary that all the tests can be employed for all strains, that test reproducibility is high and that the majority of phena show a low overlap with other phena (Sneath, 1974, 1979). Additionally, the results of numerical taxonomic studies should be compared to other numerical surveys and to classifications based on chemical, genetical and serological data (Goodfellow, 1986).

In the present study, test reproducibility was assessed using the methods of Sneath \& Johnson (1972). Overall test error was low $(p=3.11 \%)$ and comparable to other studies $(p=3 \cdot 12 \%$, Holmberg \& Nord, $1975 ; p=1.6 \%$, Schofield \& Schaal, 1981; $p=3.7 \%$, Goodfellow \& Alderson, $1977 ; p=2 \cdot 1 \%$, Goodfellow \& Pirouz, 1982; $p=3.36 \%$, Williams et al., 1983a). Acid production from a range of sugars gave scores for the the probability of erroneous results $(p=7.09 \%)$. These tests were also difficult to read photometrically. A comparison of test results obtained visually and photometrically gave discrepancies of $7.45 \%$ for all results; however, test results of sugar fermentations showed high inconsistencies of $20.5 \%$. Often the growth of the streptomycetes resulted in the production of cell agglomerates, thus preventing a homogeneous turbidity in the test wells of the microplates and requiring that all test results be checked visually. The 20 strains chosen for the reproducibility studies (Table 1) showed marked differences in their growth characteristics, so we did not exclude tests beforehand on the basis of the triplicate results of these strains, because the extent of variation due to the high variability within the genus could not be estimated. 
Sneath \& Johnson (1972) recommended that tests with more than $10-15 \%$ error should be rejected. In our study, these values were only observed for a few sugar acidification tests. As already mentioned, inconsistencies and instabilities in tests for all strains were noted, and only those tests showing a high degree of stability for all strains were chosen for identification purposes (Kämpfer \& Kroppenstedt, 1991). The assessment of cluster overlap revealed some overlap between the phena, confirming earlier numerical taxonomic studies (Gyllenberg, 1970; Gyllenberg et al., 1967; Paszkiewicz, 1972). The overlap of clusters can be explained by the high number of test organisms, the high number of tests, and the physiological heterogeneity of strains within the genus Streptomyces. In the study of Williams et al. (1983a), only slight overlap was found between the phena. As pointed out by these authors, the overlap may reflect inadequacies in some of the tests in the analysis. The overlap of clusters 30 and 31 containing $S$. albus and related strains, 35 and 36 containing $S$. rimosus strains, and 51,53 to 57 with $S$. violaceusniger and related organisms may indicate that these clusters represent one cluster. This is supported by the UPGMA $/ S_{\mathrm{J}}$ analysis.

Well-defined clusters showed only low or no overlap with other phena, thus confirming in part the results of Williams et al. (1983a). The majority of overlaps were found in pairs of clusters containing only a few strains and in those which could not be recovered in the UPGMA/ $S_{\mathrm{J}}$ analysis. As pointed out by Williams et al. $(1983 a)$, it would be unrealistic to demand very low values given the present state of streptomycete taxonomy.

The result of numerical phenetic studies have to be interpreted carefully and the results of different statistics have to be considered, including the use of different similarity coefficients and calculations of overlaps between the phena to determine the content and quality of the classifications.

The choice of the similarity coefficient has a marked influence on cluster composition. The Jaccard coefficient $\left(S_{\mathrm{J}}\right)$ considers exclusively positive matches. Therefore, the composition of clusters created with this coefficient depends on the number of positive test results for the strains. Isolates which show a majority of negative test results can be grouped into a single cluster using the simple-matching coefficient, $S_{\mathrm{SM}}$ whereas this grouping can be considerably changed using the Jaccard coefficient. The delineation of clusters and subclusters at the $81.5 \%\left(S_{\mathrm{SM}}\right)$ and the $84.0 \%\left(S_{\mathrm{SM}}\right) \mathrm{S}$-levels is arbitrary, but was chosen because several clusters of the study of Williams et al. (1983a) could be recovered, in addition to the agreement of groups defined by other studies (Korn et al., 1978; Mordarski et al., 1986; Ridell et al., 1986).

Although it is the standard practice in numerical phenetics to use a single cut-off point in each analysis, it is obvious that in the UPGMA $/ S_{\mathrm{M}}$ analysis some closely related strains were assigned to different clusters (clusters 30, 31 and 32, S. albus and related strains; clusters 35 and $36, S$. rimosus; clusters 51 and 53 to $57, S$. violaceusniger and $S$. hygroscopicus). A delineation in a staggered line, as used by Priest et al. (1988) in their UPGMA $/ S_{\mathrm{J}}$ analysis of Bacillus species, is probably a better way to define phena in numerical surveys with large numbers of strains and tests.

The number of tests employed in our study is unusually high. Although an upper limit of 200 characters has been recommended (O'Brien \& Colwell, 1987) this is not stringent, and an argument in favour of using large numbers of tests is that the effects of plasmid-borne traits will be relatively small (Harwood, 1980). There is no universal criterion of the goodness of a classification (Sneath \& Sokal, 1973), but in numerical phenetics a classification is considered to be optimal if it represents as closely as possible the original similarity matrix among the OTUs. This can be estimated by the calculation of cophenetic correlations (Sneath \& Sokal, 1973). In our study, values for cophenetic correlation coefficients of 0.6929 and 0.8239 were found for the UPGMA $/ S_{\mathrm{J}}$ and UPGMA $/ S_{\mathrm{SM}}$ classifications, respectively. It has generally been found that $r_{\mathrm{cs}}$ values vary from 0.6 to 0.95 (Sneath \& Sokal, 1973).

As pointed out by Williams et al. (1989), streptomycete taxonomy should be improved by the application of statistical methods for the significance and integrity of groups and their application to all validly published species. Furthermore, the correlation between numerical phenetic, chemotaxonomic and molecular genetic data has to be assessed. Chemotaxonomic methods have to be improved for the analysis of quantitative data (O’Donnell, 1988; Williams et al., 1989).

Cluster-groups as defined by Williams et al. (1983a) in their UPGMA $/ S_{\mathrm{SM}}$ analysis could not be detected in our analysis, although some parallels to some of these groups were observed. Taking a similarity level of $79.5 \%\left(S_{\mathrm{SM}}\right)$, it can be seen from Fig. 1 that the clusters 1 to 17 fell within a cluster-group containing the majority of strains (223 to 280 inclusive) assigned to cluster group A of Williams et al. (1983a). The clusters 33 to 36 , joined at the $79.5 \%\left(S_{\mathrm{SM}}\right)$ similarity level of our analysis, representing $S$. alboflavus, $S$. rimosus and related organisms, shared many features with cluster group B of Williams $e t$ al. $(1983 a)$, which consisted of the major cluster $S$. rimosus and the single-member cluster $S$. amakusaensis. Similar groupings to cluster groups C, D, and E of Williams et al. (1983a) could not be detected in our numerical survey. Clusters 22 to 26 contained most of the strains grouped within cluster groups $F$ and $G$ of Williams et al. (1983a). Only a few strains of cluster 
groups H, I, and J of Williams et al. (1983a) were included in our study. These strains formed singlemember clusters 19 and 87 , or fell within subclusters 22-1, 22-3 and 22-4, and clusters 4 and 51. The clustergroups of Williams et al. (1983a) could not be recovered in their UPGMA $/ S_{\mathrm{J}}$ analysis, and were not found in our UPGMA $/ S_{\mathrm{J}}$ analysis.

There seems to be no reason to doubt the species status of the $S_{\mathrm{SM}}$ subcluster 1-1 (S. albidoflavus, Williams group A1A), subcluster 1-2 (S. halstedii, Williams group A1C) and subcluster 1-3 (S. griseus, Williams group A1B). These groups were also found in earlier studies (Kutzner et al., 1978; Kurylowicz et al., 1970, 1975). Studies of DNA-DNA homology have confirmed the species status of these subclusters: the Williams clusters A1A, $\mathrm{A} 1 \mathrm{~B}$ and $\mathrm{AlC}$ were recovered, with some changes, in the studies of Mordarski et al. (1986). The strains S. albidus ISP 5320 and $S$. alboniger ISP 5043 were not assigned to the Williams group 1A (subcluster $1-1$ of the present investigation), but were found in subcluster 1-3 and subcluster 1-7, respectively. S. olivaceus ISP 5072 was recovered as single-member cluster 42 and was not included in subcluster 1-4, which contained four strains of $S$. olivaceus. This subcluster overlapped with subcluster 1-2 ( $S$. halstedii) at a $V_{\mathrm{G}}$ level of $16 \%$, thus showing the strong relationship suggested by Williams $e t$ al. (1983a). Mordarski et al. (1986) could not assign $S$. olivaceus ISP 5072 to the expected DNA-homology group. The DNA homology results of Mordarski et al. (1986), who assigned $S$. coelicolor, $S$. albidoflavus, $S$. albidus, $S$. alboniger, $S$. canescens, 'S. citreus', $S$. fellus, $S$. limosus, $S$. rutgersensis and $S$. sampsonii to one taxon, and $S$. alboviridis, 'S. cratifer' and ' $S$. oligocarbophilus' to another genospecies, are strongly supported by our phenetic data. The $S$. coelicolor group of Korn et al. (1978), containing 28 strains, was found in subcluster 1-1 (S. albidoflavus) with the exception of the one strain $S$. coelicolor DSM 40674, which formed the single-member cluster 86.

Of the 30 strains of the $S$. griseus group investigated by Korn et al. (1978), 24 were recovered in subcluster 1-3 ( $S$. griseus) of the present study, two strains in subcluster 223 (S. chryseus DSM 40420 and $S$. vinaceus DSM 40515) and one in each of subcluster 1-1 (S. badius DSM 40138), cluster 6 (S. baarnensis DSM 40232) and single-member cluster 47 ( $S$. anulatus DSM 40361). Many strains of subcluster 1-2 ( $S$. griseus) have been shown to comprise a distinct DNA homology group (Okanishi et al., 1972) and the results are in line with the earlier studies of Hütter (1967) and Gyllenberg (1970).

Subclusters 1-5 (S. antibioticus), 1-6 (S. flaveolus) and 17 (S. diastatochromogenes) have no corresponding phena in the study of Williams et al. (1983a). Strains grouped into the heterogeneous cluster A31 (S. antibioticus) of
Williams et al. (1983a) were found in all three subclusters, which overlapped with each other at $V_{\mathrm{G}}$ levels of 10 to $13 \%$.

The recognition of cluster 2 (S. exfoliatus, Williams groups A5 and A6) supports the view of Williams et al. $(1983 a)$, who found considerable overlap between their phena A5 (S. exfoliatus) and A6 ( $S$. violaceus), that these strains should be grouped within one species. Cluster 6 ( $S$. rochei) was a large cluster containing the majority of strains of Williams groups A12 ( $\mathrm{S}$. rochei) and A21 ( $\mathrm{S}$. griseoruber) in addition to many strains of the grey series of category IV (Williams et al., 1989). The $S$. violaceoruber group defined by phage typing (Korn et al., 1978) was recovered in this cluster, with the exception of the type strain $S$. violaceoruber DSM $40049^{\mathrm{T}}$, which was shown to be physiologically different (Tables 2 to 4 ) and therefore grouped inio cluster 69. Cluster 6 also contained ' $S$. lividans', thus showing the relationship between this strain and strains of $S$. violaceoruber, and confirming group IV of the numerical survey of Kurylowicz et al. (1975). Cluster 7 (S. prasinopilosus) contained the majority of strains found in Williams group A37. This cluster was recovered in the UPGMA/ $S_{\mathrm{J}}$ analysis and all strains produced ornamented, green spores in RA or SP chains and were melanin negative. Cluster 9 ( $S$. violaceus) was composed of the majority of strains from Williams groups A18 (S. cyaneus) and A19 (S. diastaticus). These clusters showed no overlap in the study of Williams et al. (1983a), but they overlapped with Williams cluster A40, from which one strain was also found in our cluster 9. Most of the strains found in cluster 9 produced blue, spiny spores. Strains of the $S$. viridochromogenes group studied by phage typing (Korn et al., 1978) were found within cluster 9 (S. violaceus). Many strains of $S$. chartreusis were also found in this cluster, thus confirming the relationship between these species (Hütter, 1967). With the exception of five strains, this cluster contained all the $S$. cyaneus strains studied by Saddler et al. (1987) in their investigations using quantitative fatty acid data. Their grouping was not supported by our investigation. The significance of fatty acid data in defining taxa at the species level remains unresolved. Cluster 9 also contained three strains of $S$. scabies and may be the home for the majority of strains studied by Lambert \& Loria $(1989 a, b)$.

The majority of strains of the genus Streptoverticillium were found in subcluster $22-1$ of the UPGMA $/ S_{\mathrm{SM}}$ study. This cluster was also recovered in the UPGMA $/ S_{\mathrm{J}}$ analysis. Only three strains belonging to the genus Streptoverticillium are found in other clusters: $S v$. lavenduligriseum ISP 5487 in subcluster 1-5; 'Sv. luteoreticuli' ISP 5509 in subcluster 1-8; and Sv. albireticuli ISP 5051 in cluster 76 . The other strains were physiologically homogeneous. The groups obtained in the study of Locci 
et al. (1981) could not be recovered in the present investigation. It is widely accepted that the genera Streptomyces and Streptoverticillium are closely related. Both have cell-wall type I (Lechevalier \& Lechevalier, $1970 a, b$ ), a high content of GC in their DNA (Pridham \& Tresner, 1974a, b) and high similarities in DNA homology (Okanishi \& Gregory, 1970; Okanishi et al., 1972; Kroppenstedt et al., 1981), and are lysed by the same phages (Prauser, 1976; Wellington \& Williams, 1981). Additionally, they contain similar menaquinones, fatty acids and polar lipids (Collins et al., 1977; Kroppenstedt \& Kutzner, 1976; Lechevalier et al., 1977). Immunodiffusion studies (Ridell et al., 1986) further support the affinity between the two genera, especially the relationship between streptoverticillia and Streptomyces lavendulae. This relationship was confirmed in our study, as the subclusters 22-1 ( $\mathrm{Sv}$. griseocarneum) and 22-3 ( $S$. lavendulae) were shown to be phenetically closely related as indicated by their degree of overlap. As pointed out by Kutzner (1981) and confirmed here (Table 2), the streptoverticillia utilize only a limited number of carbon sources. The only support for distinguishing between Streptomyces and Streptoverticillium has come from DNA-RNA pairing studies (Gladek et al., 1985). The current recognition of Streptoverticillium as a separate genus is nonetheless still a practical proposition. The basis of the classification within the genus Streptoverticillium (Locci et al., 1981) could not be confirmed in the present study.

Subcluster 22-3 (S. lavendulae) was heterogeneous and was split into several clusters in the UPGMA $/ S_{\text {J }}$ analysis. However, strains of $S$. lavendulae and synonyms were physiologically homogeneous and were found largely in $S_{\mathrm{J}}$ cluster 42 . The strong relationship of $S$. lavendulae to streptoverticillia shown by serological studies (Ridell et al., 1986) was confirmed. Subcluster 22-5 ( $S$. fradiae) was also found to be related to $S$. lavendulae and Streptoverticillium strains, thus supporting the findings of Williams et al. (1983a).

Cluster 25 (S. lydicus) contained seven strains of the $S$. lydicus phenon (A29) of Williams et al. (1983a). This cluster additionally contained four strains of $S$. hygroscopicus, currently regarded as a synonym of $S$. violaceusniger. These strains were different from those strains grouped into clusters 53 to 57 . It is obvious that strains designated as $S$. hygroscopicus are heterogeneous, and this is confirmed by fatty acid data (R. M. Kroppenstedt, unpublished).

Cluster 30 ( $S$. albus) was a homogeneous phenon, showing high similarities to the overlapping clusters 31 and 32 , which also contained strains of $S$. albus or synonyms. These results are in accordance with Korn et al. (1976) and Williams et al. (1989). Taking a similarity level of $79.5 \%\left(S_{\mathrm{SM}}\right)$, clusters 30 to 32 formed a relatively distinct group, separate from other Streptomyces species, thus confirming the isolated position of the type species of the genus, which can be further demonstrated by its temperature range, pathogenicity and fatty acid data (R. M. Kroppenstedt, unpublished).

Strains grouped within cluster 33 (S. alboflavus) which showed a strong relationship to those found in cluster 35 ( $S$. rimosus) were grouped into subcluster 1-33 of the UPGMA $/ S_{\mathrm{J}}$ analysis. They were morphologically very similar, most of them producing a white or yellow spore mass and no melanin. Cluster 35 ( $S$. rimosus) was also found by Williams et al. (1983a), in their cluster group B, distant from other streptomycetes, which was confirmed in our study.

Strains of $S$. violaceusniger and S. hygroscopicus were found in the related and overlapping clusters 51 and 53 to 57 , confirming the results of Williams et al. (1983a). As mentioned above, a delineation of the dendrogram in a staggered line would have clustered these strains together.

A comparison of major phena of the UPGMA $/ S_{\mathrm{SM}}$ analysis with the corresponding clusters of the study of Williams et al. $(1983 a)$ is given in Table 5. Although in some cases it was difficult to define corresponding clusters, of 215 strains, $164(76.3 \%)$ were found in comparable phena. Predominant character states of morphological features are also in line with the study of Williams et al. (1983a) (see Table 9 of that study). The species status of the minor clusters in our analysis is difficult to evaluate, because most of them contained strains grouped into different clusters of Williams et al. (1983a). Their taxonomic position will require confirmation by genetical, improved chemotaxonomic and serological data.

With respect to morphological criteria, our study supports the view of Williams et al. (1983a) that these traditional criteria, which are often difficult to determine (Gottlieb \& Shirling, 1967; Williams \& Wellington, 1980; Kutzner, 1981), are inadequate for classification. Although streptomycetes producing a blue spore mass were largely grouped together (cluster $9, S$. violaceus), Streptomyces species producing a green, red or violet spore mass were found in different clusters, thus confirming the studies of Kurylowicz et al. (1975) and Williams et al. (1983a). The classification of 'species' currently classified within category IV of Williams et al. (1989) is improved by allocation of most of these strains to major clusters of the UPGMA $/ S_{\mathrm{SM}}$ analysis. Physiological characters are therefore indispensable for classification and identification of Streptomyces. Carbon source utilization tests were often used in earlier taxonomic studies. The utilization of carbohydrates such as sucrose, $\mathrm{L}$-arabinose, $i$-inositol, mannitol, rhamnose and raffinose was recommended as an aid in species differentiation in 
the International Streptomyces Project (Shirling \& Gottlieb, 1966; 1968 a, b, 1969, 1972) and these tests are included in species descriptions in Bergey's Manual of Determinative Bacteriology, 8th edn (Pridham \& Tresner, $1974 b$ ). The differentiation potential of these tests, especially the utilization of different polyalcohols, can be readily seen from Tables 2 to 4 . The use of qualitative enzyme tests for differentiation of streptomycetes has been recently applied to 88 strains belonging to different species-groups of Streptomyces (Goodfellow et al., 1987). These tests were performed as growth-independent hydrolysis tests of fluorogenic, methylumbelliferyllinked substrates. In comparison to our growth-dependent enzyme tests with nitrophenyl- or nitroanilidelinked substrates, several corresponding results, but also some differences, were observed. All or the majority of strains were able to produce acid and alkaline phosphatases, $\alpha$-D-glucosidase, $\beta$-D-glucosidase and $N$-acetyl- $\beta$-Dglucosaminidase, which was also found by Goodfellow $e t$ al. (1987). Even the ability of $\beta$-L-fucosidase to differentiate between $S$. albidoflavus and $S$. griseus ( $S$. anulatus) could be observed. In contrast to the results of Goodfellow et al. (1987), not all strains produced $\beta$-Dgalactosidase, and other profiles were obtained for $\beta$-Dglucuronidase and sulphatase production. It seems that these tests may be important for characterization of streptomycetes, but cannot be used for their unambiguous identification. Quantitative chemotaxonomic methods have also been applied to some species of the genus Streptomyces (O'Donnell, 1988), but the differentiation potential of quantitative fatty acid data at the species level remains questionable (Saddler et al., 1987).

Our study showed that many strains of the genus Streptomyces deposited in culture collections are poorly characterized and assigned to the wrong species. As an example, the three strains of $S$. albus found in subcluster 1-1 (S. albidoflavus) were shown by fatty acid profiles to belong to $S$. albidoflavus (R. M. Kroppenstedt, unpublished). In contrast, several strains of the same species were found in different phena, because of their atypical physiological reactions. Examples are $S$. anulatus ISP $5361^{\mathrm{T}}$, which was not grouped into subcluster 1-3 ( $S$. griseus) as expected, but formed single-member cluster 47 , and S. violaceoruber ISP $5049^{\mathrm{T}}$, which was not found in cluster $6(S$. rochei), but in cluster 69 (S. ochraceiscleroticus). The reasons for the atypical physiological patterns of such strains are difficult to determine. Test conditions, for example the time of reading of tests, incubation temperatures, test media, and the source and size of inoculum may not be optimal for all test strains, or the deviations may be due to mutations. As pointed out by Hill (1974), numerical taxonomy should be regarded as any other theory, and as such should be modified and refined by further testing, including the use of genetical methods.

\section{Conclusions}

This study confirms the extensive species reduction within the genus Streptomyces proposed by Williams et al. (1983a) and supports the view of Sneath (1970) that numerical analysis is a practical way of dealing with the overspeciation in the genus. The taxonomic position of many strains currently classified within species category IV (Williams et al., 1989) could be evaluated (Table 1). The results of our study indicate that the genus Streptomyces is still overspeciated.

There is evidence that the major subclusters and major clusters $S$. albidoflavus, $S$. halstedii, $S$. griseus, $S$. exfoliatus, $S$. rochei, $S$. lydicus, $S$. fradiae, $S$. lavendulae, $S$. rimosus and $S$. albus can be regarded as species, in addition to $S$. violaceusniger, strains of which were found in closely related and overlapping clusters. The taxonomic status of these species is supported by other taxonomic methods. Our results support the view that strains placed in the genus Streptoverticillium should be reclassified into the genus Streptomyces. However, the species status of many phena, including the minor clusters and single-member clusters, remains questionable. Species grouped into category III and category IV of Williams et al. (1989) should be regarded as in a state of transition until further genetical information is available. The nomenclature for phena used in our study was according to the procedure proposed by Williams $e t$ al. $(1983 a)$. Any necessary nomenclatural changes will be published elsewhere.

\section{Note added in proof}

Since the completion of this study the formal proposal has been made to unify the genera Streptoverticillium and Streptomyces in an amended genus Streptomyces based on phylogenetic similarities (Witt \& Stackebrandt, 1990). In this study and in a further study by Labeda \& Lyons (1991), extensive DNA-DNA hybridizations were carried out on members of the Williams clusters A21 ( $S$. griseoruber), F61 (S. lavendulae) and A18 ( $S$. cyaneus). In both studies, the hybridization values spanned a wide range, and in most cases intracluster pairing values were as low as those found between members of different Williams clusters. In some cases several strains grouped within the same cluster by Williams et al. (1983a), and showing very low intracluster hybridization values, were not grouped into the corresponding cluster in our study (e.g. Williams cluster A21: $S$. griseoruber ISP $5281^{\mathrm{T}}, S$. griseoruber ISP $5275^{\mathrm{T}}$; Williams group F61: $S$. xanthophaeus ISP 5134'; 
Williams group A18: S. amakusaensis ISP 5219,$S$. fumanus ISP $5154^{\mathrm{T}}$ and $S$. griseochromogenes ISP $5499^{\mathrm{T}}$ ). However, as pointed out by Witt \& Stackebrandt (1990) it is not possible to determine the phylogenetic depth of the phenetic clusters solely by DNA-DNA hybridization, and the question about their phylogenetic coherency cannot be answered at present.

\section{References}

Alderson, G., Athalye, M. \& White, R. P. (1984). Numerical methods in the taxonomy of Sporoactinomycetes. In Biological, Biochemical and Biomedical Aspects of Actinomycetes, pp. 597-615. Edited by L. Ortiz-Ortiz, L. F. Bojalil \& V. Yakoleff. London: Academic Press.

Austin, B. \& Colwell, R. R. (1977). Evaluation of some coefficients for use in numerical taxonomy of micro-organisms. International Journal of Systematic Bacteriology 27, 204-210.

Collins, M. D. \& JonEs, D. (1981). Distribution of isoprenoid quinone structural types in bacteria and their taxonomic implications. Microbiological Reviews 45, 316-354.

Collins, M. D., Pirouz, Y., Goodfellow, M. \& Minnikin, D. E. (1977). Distribution of menaquinones in actinomycetes and corynebacteria. Journal of General Microbiology 100, 221-230.

Fierro, J. F., Parra, F., Quiros, L. M., Hardison, C. \& Salas, J. A. (1987). Heterogeneity of the ribosomal protein pattern in mycelium of Streptomyces species. FEMS Microbiology Letters 41, 283-287.

Gause, G. F., Preoprazhenskaya, T. P., Kudrina, E. S., Blinov, N. O., Ryabova, I. D. \& Sveshnikova, M. A. (1957). Problems in the Classification of Antagonistic Actinomycetes. Moscow: State Publishing House of Medical Literature, Medzig.

Gladek, A., Mordarski, M., Goodfellow, M. \& Williams, S. T. (1985). Ribosomal ribonucleic acid similarities in the classification of Streptomyces. FEMS Microbiology Letters 26, 175-180.

GoODFELlOW, M. (1986). Actinomycetes systematics: present state and future prospects. In Biological, Biochemical and Biomedical Aspects of Actinomycetes, Part B, pp. 487-496. Edited by G. Szabo, S. Biro \& M. Goodfellow. Budapest: Academiai Kiado.

Goodfellow, M. \& Alderson, G. (1977). The actinomycete genus Rhodococcus: a home for the 'rhodochrous' complex. Journal of General Microbiology 100, 99-122.

Goodfellow, M. \& Cross, T. (1984). Classification. In Biology of Actinomycetes, pp. 7-164. Edited by M. Goodfellow, M. Mordarski \& S. T. Williams. London: Academic Press.

Goodfellow, M. \& PIRouz, T. (1982). Numerical classification of sporoactinomycetes containing meso-diaminopimelic acid in the cell wall. Journal of General Microbiology 128, 503-527.

Goodfellow, M. \& SCHAal, K. P. (1979). Identification methods of Nocardia, Actinomadura and Rhodococcus. In Identification Methods for Microbiologists, 2nd edn, pp. 261-276. Edited by F. A. Skinner \& D. W. Lovelock. London: Academic Press.

GoOdFellow, M. \& WAYNE, L. G. (1982). Taxonomy and nomenclature. In Biology of the Mycobacteria, vol. 1, pp. 472-521. Edited by C. Ratledge \& J. L. Stanford. London: Academic Press.

GoOdFellow, M., Mordarski, M. \& Williams, S. T. (1984). Introduction to and importance of actinomycetes. In Biology of Actinomycetes, pp. 1-6. Edited by M. Goodfellow, M. Mordarski \& S. T. Williams. London: Academic Press.

Goodfellow, M., Williams, S. T. \& Alderson, G. (1986). Transfer of Chainia species to the genus Streptomyces with emended descriptions of species. Systematic and Applied Microbiology 8, 55-60.

Goodfellow, M., Lonsdale, C., James, A. L. \& MacNamara, O. C. (1987). Rapid biochemical tests for the characterization of streptomycetes. FEMS Microbiology Letters 43, 39-44.

GotTlieb, D. \& Shirling, E. B. (1967). Cooperative description of type cultures of Streptomyces. I. The International Streptomyces Project. International Journal of Sytematic Bacteriology 17, 315-322.
GYLLENBERG, H. G. (1970). Factor analytical evaluation of patterns of correlated characteristics in streptomycetes. In The Actinomycetales, pp. 101-105. Edited by H. Prauser. Jena: Gustav Fischer Verlag. GyllenberG, H. G., WozNickA, W. \& KuRYlowicz, W. (1967). Application of factor analysis in microbiology. 3. A study of the 'yellow series' of streptomycetes. Annales Academiae Scientiarum Fennicae, Series A (IV, Biologica) 114, 3-15.

HARWOOD, C. R. (1980). Plasmids. In Microbiological Classification and Identification, pp. 27-53. Edited by M. Goodfellow \& R. G. Board. London: Academic Press.

HILL, L. R. (1974). Theoretical aspects of numerical identification. International Journal of Systematic Bacteriology 24, 494-499.

HolmberG, K. \& NoRD, C. E. (1975). Numerical taxonomy and laboratory identification of Actinomyces and Arachnia and some related bacteria. Journal of General Microbiology 91, 17-44.

HUGH, R. \& Leifson, E. (1953). The taxonomic significance of fermentative versus oxidative metabolism of carbohydrates by various gram-negative bacteria. Journal of Bacteriology 66, 22-24.

HÜTTER, R. (1967). Systematik der Streptomyceten. Basel: S. Karger.

KÄMPFER, P. \& KROPPENSTEDT, R. M. (1991) Probabilistic identification of streptomycetes using miniaturized physiological tests. Journal of General Microbiology 137, 1893-1902.

KORN, F., WEINGÄRTNER, B. \& KUTZNER, H. J. (1978). A study of twenty actinophage: morphology, serology, relationship and host range. In Genetics of the Actinomycetales, pp. 251-270. Edited by E. Freersen, I. Tarnok \& J. H. Thumin. Stuttgart: Gustave Fischer Verlag.

KROPPENSTEDT, R. M. (1985). Fatty acid and menaquinone analysis of actinomycetes and related organisms. In Chemical Methods in Bacterial Systematics, pp. 173-199. Edited by M. Goodfellow \& D. E. Minnikin. London: Academic Press.

KROPPENSTEDT, R. M. \& KUTZNER, H. J. (1976). Biochemical markers in the taxonomy of the Actinomycetales. Experientia 32, 318-319.

KROPPENSTEDT, R. M., KoRN-WendisCh, F., Fowler, V. J. \& STACKEBRANDT, E. (1981). Biochemical and molecular genetic evidence for the transfer of Actinoplanes armeniacus into the family Streptomycetaceae. Zentralblatt für Bakteriologie, Mikrobiologie und Hygiene (I Abteilung, Originale C) 2, 254-262.

Kurylowicz, W., Woznicka, W., Paszkiewicz, A. \& Malinowski, K. (1970). Application of numeric taxonomy in streptomyces. In Actinomycetales, pp. 107-122. Edited by H. Prauser. Jena: Gustav Fischer Verlag.

KuR Ylowicz, W., Paszkiewicz, A., WozNICKa, W., KurzatKowski, W. \& Szulga, T. (1975). Classification of Streptomyces by different numerical methods. Postepy Higieny i Medycyny Doswiadczalnej 29, 281-355.

KUTZNER, H. J. (1981). Streptomyces. In The Prokaryotes: a Handbook of Habitats, Isolation and Identification of Bacteria, pp. 2028-2090. Edited by M. P. Starr, H. Stolp, H. G. Trüper, A. Balows \& H. G. Schlegel. Berlin: Springer Verlag.

KutzNer, H. J., Böttinger, V. \& Heitzer, R. D. (1978). The use of physiological criteria in the taxonomy of Streptomyces and Streptoverticillium. Zentralblatt für Bakteriologie, Parasitenkunde, Infektionskrankheiten und Hygiene, I Abteilung, Supplement 6, 25-29.

LABEDA, D. P. \& LyONS, A. J. (1991). Deoxyribonucleic acid relatedness among species of the "Streptomyces cyaneus" cluster. Systematic and Applied Microbiology 14, 158-164.

LAMBERT, D. H. \& LoRIA, R. (1989a). Streptomyces scabies sp. nov., nom. rev. International Journal of Systematic Bacteriology 39, 387-392.

LAMBeRT, D. H. \& LoRIA, R. (1989b). Streptomyces acidiscabies sp. nov. International Journal of Systematic Bacteriology 39, 393-396.

Langham, C. D., Williams, S. T., SNeath, P. H. A. \& Mortimer, A. M. (1989). New probability matrices for identification of Streptomyces. Journal of General Microbiology 135, 121-133.

LeCheVAlier, H. A. \& Lechevalier, M. P. (1970a). A critical evaluation of the genera of aerobic actinomycetes. In The Actinomycetales, pp. 393-405. Edited by H. Prauser. Jena: Gustav Fischer Verlag.

Lechevalier, M. P. \& Lechevalier, H. A. (1970 $b$ ). Chemical composition as a criterion in the classification of aerobic actinomycetes. International Journal of Systematic Bacteriology 20, 435-443. 
Lechevalier, M. P., Debievre, C. \& Lechevalier, H. A. (1977). Chemotaxonomy of aerobic actinomycetes: phospholipid composition. Biochemical Systematics and Ecology 5, 249-260.

LoCCI, R. \& SCHOFIELD, G. M. (1989). Genus Streptoverticillium (Baldacci 1958) 15, emend. mut. char. Baldacci, Farina and Locci 168 AL. In Bergey's Manual of Systematic Bacteriology, pp. 2492-2504. Edited by S. T. Williams, M. Sharpe \& J. G. Holt. Baltimore: Williams \& Wilkins.

Locci, R., Rogers, J., Sardi, G. \& Schofield, G. M. (1981). A preliminary numerical study on named species of the genus Streptoverticillium. Annali di Microbiologia 31, 115-121.

MinNikin, D. E. \& GoodfELlow, M. (1980). Lipid composition in the classification and identification of acid-fast bacteria. In Microbiological Classification and Identification, pp. 189-256. Edited by $M$ Goodfellow \& R. G. Board. London: Academic Press.

Minnikin, D. E., Pirouz, T. \& Goodfellow, M. (1977). Polar lipid composition in the classification of some Actinomadura species. International Journal of Systematic Bacteriology 27, 118-121.

Minnikin, D. E., Goodfellow, M. \& Collins, M. D. (1978). Lipid composition in the classification and identification of coryneform and related taxa. In Coryneform Bacteria, pp. 85-160. Edited by I. J. Bousfield \& A. G. Callely. London: Academic Press.

MoRdarski, M., Goodfellow, M., Williams, S. T. \& SNeath, P. H. A. (1986). Evaluation of species groups in the genus Streptomyces. In Biological, Biochemical and Biomedical Aspects of Actinomycetes, Part B, pp. 517-525. Edited by G. Szabo, S. Biro \& M. Goodfellow. Budapest: Academiai Kiado.

O'BRIEN, M. \& COLWELl, R. R. (1987). Characterization tests for numerical taxonomy studies. Methods in Microbiology 19, 69-104.

O'DoNNELL, A. (1988). Recognition of novel actinomycetes. In Actinomycetes in Biotechnology, pp. 69-88. Edited by M. Goodfellow, S. T. Williams \& M. Mordarski. London: Academic Press.

OKANISHI, M. \& GREGoRY, K. F. (1970). Methods for the determination of deoxyribonucleic acid homologies in Streptomyces. Journal of Bacteriology 104, 1086-1094.

OKANISHI, M., AGaKaWA, H. \& UmezaWa, H. (1972). An evaluation of taxonomic criteria in streptomycetes on the basis of deoxyribonucleic acid homologies. Journal of General Microbiology 72, 49-58.

PASZKIEwICZ, A. (1972). Application of numerical methods to the taxonomy of the genus Streptomyces. Archivum Immunologiae et Therapiae Experimentalis 20, 307-332.

PraUser, H. (1976). Host-phage relationship in nocardioform organisms. In The Actinomycetales, pp. 266-284. Edited by M. Goodfellow, G. H. Brownell \& J. A. Serrano. London: Academic Press.

PridHAM, T. G. (1976). Contemporary species concepts in the Actinomycetales. In Actinomycetes: The Boundary Microorganisms, pp. 163-174. Edited by T. Arai. Tokyo: Toppan Co.

Pridham, T. G. \& TRESNeR, H. D. (1974a). Family VII. Streptomycetaceae Waksman and Henrici 1943, 339. In Bergey's Manual of Determinative Bacteriology, 8th edn, pp. 747-748. Edited by R. E. Buchanan \& N. E. Gibbons. Baltimore: Williams \& Wilkins.

Pridham, T. G. \& Tresner, H. D. (1974b). Streptomyces Waksman and Henrici 1943, 339. In Bergey's Manual of Determinative Bacteriology, 8th edn, pp. 748-829. Edited by R. E. Buchanan \& N. E. Gibbons. Baltimore: Williams \& Wilkins.

Pridham, T. G., Hesseltine, C. \& Benedict, R. G. (1958). A guide for the classification of streptomycetes according to selected groups. Placement of strains in morphological sections. Applied Microbiology 6, 52-79.

Priest, F. G., Goodfellow, M. \& Todd, C. (1988). A numerical classification of the genus Bacillus. Journal of General Microbiology 134, 1847-1882.

Ridell, M., Wallerström, G. \& Williams, S. T. (1986). Immunodiffusion analysis of phenetically defined strains of Streptomyces, Streptoverticillium and Nocardiopsis. Systematic and Applied Microbiology 8, 24-27.

SADDler, G. S., O'Donnell, A. G., Goodfellow, M. \& Minnikin, D. E. (1987). SIMCA pattern recognition in the analysis of streptomycete fatty acids. Journal of General Microbiology 133, $1137-$ 1147.
Schofield, G. M. \& SchaAl, K. P. (1981). A numerical taxonomic study of members of the Actinomycetaceae and related taxa. Journal of General Microbiology 127, 237-259.

SHIRLING, E. B. \& GotTLIEB, D. (1966). Methods for characterization of Streptomyces species. International Journal of Systematic Bacteriology 16, 313-340.

ShIRLING, E. B. \& GotTlieB, D. (1968a). Cooperative description of type cultures of Streptomyces. II. Species descriptions from first study. International Journal of Systematic Bacteriology 18, 69-118.

ShiRling, E. B. \& GotTlieb, D. $(1968 b)$. Cooperative description of type cultures of Streptomyces. III. Additional species descriptions from first and second studies. International Journal of Systematic Bacteriology 18, 279-391.

Shirling, E. B. \& GotTlieb, D. (1969). Cooperative description of type cultures of Streptomyces. IV. Species descriptions from the second, third and fourth studies. International Journal of Systematic Bacteriology 18, 391-512.

ShIRLING, E. B. \& GotTlieb, D. (1972). Cooperative description of type cultures of Streptomyces. V. Additional descriptions. International Journal of Systematic Bacteriology 22, 265-394.

Skerman, V. B. D., McGowan, V. \& Sneath, P. H. A. (1980). Approved lists of bacterial names. International Journal of Systematic Bacteriology 30, 225-420.

SNEATH, P. H. A. (1970). Application of numerical taxonomy to Actinomycetales: problems and perspectives. In the Actinomycetales, pp. 371-378. Edited by H. Prauser. Jena: Gustav Fischer Verlag.

SNEATH, P. H. A. (1974). Test reproducibility in relation to identification. International Journal of Systematic Bacteriology 24 508-523.

SNEATH, P. H. A. (1979). BASIC program for a significance test for two clusters in Euclidean space as measured by their overlap. Computers and Geosciences 5, 143-155.

SNEATH, P. H. A. \& Johnson, R. (1972). The influence on numerical taxonomic similarities of errors in microbiological tests. Journal of General Microbiology 72, 377-392.

SNEATH, P. H. A. \& SOKAL, R. R. (1973). Numerical Taxonomy. The Principles and Practice of Numerical Classification. San Francisco: W. H. Freeman.

STACKebRandT, E. (1986). The significance of "wall types" in phylogenetically based taxonomic studies on Actinomycetes. In Biological, Biochemical and Biomedical Aspects of Actinomycetes, Part B, pp. 497-506. Edited by G. Szabo, S. Biro \& M. Goodfellow. Budapest: Academiai Kiado.

STACKEBRANDT, E. \& WOESE, C. R. (1981). The evolution of prokaryotes. Symposia of the Society for General Microbiology 32, $1-31$.

Stackebrandt, E., WunNer-Füssl, B., Fowler, V. J. \& SChleifer, K. H. (1981). Deoxyribonucleic acid homologies and ribosomal ribonucleic acid similarities among sporeforming members of the order Actinomycetales. International Journal of Systematic Bacteriology 31, 420-431.

Wellington, E. M. H. \& Williams, S. T. (1981). Host ranges of phage isolated to Streptomyces and other genera. Zentralblatt für Bakteriologie, Mikrobiologie und Hygiene (I Albteilung), Supplement 11, 93-98.

Williams, S. T. \& Wellington, E. M. H. (1980). Micromorphology and fine structure of actinomycetes. In Microbiological Classification and Identification, pp. 139-165. Edited by M. Goodfellow \& R. G. Board. London: Academic Press.

Williams, S. T., Goodfellow, M., Alderson, G., Wellington, E. M. H., SNeATh, P. H. A. \& SACKIN, M. J. (1983a). Numerical classification of Streptomyces and related genera. Journal of General Microbiology 129, 1743-1813.

Williams, S. T., Goodfellow, M., Wellington, E. M. H., Vickers, J. C., Alderson, G., Sneath, P. H. A., Sackin, M. J. \& Mortimer, A. M. $(1983 \mathrm{~b})$. A probability matrix for identification of some streptomycetes. Journal of General Microbiology 129, 1815-1830.

Williams, S. T., Locci, R., Vickers, J. C., SCHOField, G. M., SNEATH, P. H. A. \& MORTIMER, A. M. (1985). Probabilistic identification of Streptoverticillium species. Journal of General Microbiology 131, 1681 1689. 
Williams, S. T., Goodfellow, M. \& Alderson, G. (1989). Genus Streptomyces (Waksman \& Henrici, 1943) 339AL. In Bergey's Manual of Systematic Bacteriology, vol. 4, pp. 2452-2492. Edited by S. T. Williams, M. E. Sharpe \& J. G. Holt. Baltimore: Williams \& Wilkins.
WitT, D. \& STACKebRandT, E. (1990). Unification of the genera Streptoverticillium and Streptomyces, and amendation of Streptomyces Waksman and Henrici 1943, 339 AL. Systematic and Applied Microbiology 13, 361-371. 